\title{
Studying complex surface dynamical systems using helium-3 spin-echo spectroscopy
}

\author{
Barbara Angelika Johanna Lechner
}

\author{
Gonville \& Caius College \\ University of Cambridge
}

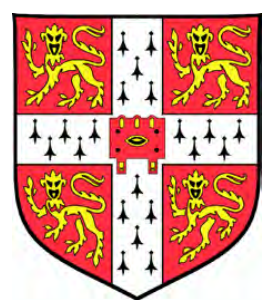

This dissertation is submitted for the degree of

Doctor of Philosophy

August 2012 



\begin{abstract}
The aim of the present thesis is to extend the range of application of the helium-3 spin-echo (HeSE) technique to complex surface dynamical systems. A twofold approach is presented: the development of improved experimental equipment and the investigation of a series of prototypical aromatic adsorbate systems. Chapter 1 discusses the motivation for this work and compares HeSE with other techniques probing surface motion. Subsequently, an introduction to the HeSE method is given in Chapter 2, explaining the theoretical background and describing the main components of the Cambridge spectrometer as well as the principle data aquisition and interpretation methods.
\end{abstract}

Chapters 3 and 4 describe the development and testing of two new pieces of equipment. MiniScat, a compact helium atom scattering apparatus, has been designed as a sample preparation facility to enable structural studies and increase the experimental throughput. A new supersonic helium beam source improves the resolution of the HeSE spectrometer by a factor of 5.5 and extends accessible timescales into the nanometre range.

The dynamics of cyclopentadienyl $(\mathrm{Cp})$ on $\mathrm{Cu}(111)$ are presented in Chapter 5 . The ionically bound $\mathrm{Cp}$ is remarkably mobile, moving in single jumps between adjacent hollow sites over an energy barrier of $41 \pm 1 \mathrm{meV}$. The data exhibit multicomponent lineshapes that allow the determination of the energy difference between fcc and hcp sites of $12.3 \pm 0.3 \mathrm{meV}$ in a Bayesian method probing the probability space of all data combined. Molecular dynamics (MD) simulations provide a friction coefficient of $2.5 \pm 0.5 \mathrm{ps}^{-1}$.

Chapter 6 shows that a physisorbed pyrrole hops between bridge sites on $\mathrm{Cu}(111)$. Strong lateral interactions alter the lineshapes from a predicted double exponential towards an apparent single exponential decay. First principles density functional theory calculations by Sacchi and Jenkins reveal that a large contribution to the experimentally determined apparent activation energy of $53 \pm 4 \mathrm{meV}$ arises from a site-dependence in the zero point energies, primarily of the vibrational $\mathrm{C}-\mathrm{H}$ and $\mathrm{N}-\mathrm{H}$ out-of-plane bending and ring torsion modes which are not directly involved in the diffusion process.

The surface dynamics of thiophene/ $\mathrm{Cu}(111)$ are investigated in Chapter 7 . Thiophene adsorbs on top sites and forms a covalent $\mathrm{S}-\mathrm{Cu}$ bond. Two competing activated processes manifest themselves in a kink in the Arrhenius plot: jump diffusion between adjacent top sites over a barrier of $59 \pm 4 \mathrm{meV}$ and rotation around the $\mathrm{S}-\mathrm{Cu}$ anchor point over a barrier of $22 \pm 2 \mathrm{meV}$. In addition, vertical motion relative to the surface is observed. MD simulations of the diffusive motion reveal an exceptionally high friction of $5 \pm 2 \mathrm{ps}^{-1}$. 


\section{Declaration}

This dissertation is the result of my own work and contains nothing which is the outcome of work done in collaboration with others except where specifically indicated in the text. It does not exceed the word limit prescribed by the Degree Committee for the Faculty of Physics and Chemistry, and has not been submitted in whole or part for a degree, diploma or other qualification at this or any other university. 


\section{Acknowledgements}

I would like to express my sincere gratitude to my supervisor Dr John Ellis for his guidance, advice and encouragement. His seemingly limitless enthusiasm for scientific matters was a great source of motivation throughout my time in this group. Likewise, I am indebted to Dr Bill Allison who always took the time to support and advise me in my endeavours.

Special thanks go to Dr Andrew Jardine for his help and advice on the design and construction of MiniScat and the new source, as well as to Dr Holly Hedgeland who instructed and supported me during my first attempts at measuring and analysing data. Furthermore, I would like to thank my second supervisor Dr Jacqui Cole for her encouragement.

Outside the group, I am indebted to Prof. Jane Hinch - initiator of the study of the dynamics of five-membered molecules - for her help, advice and many illuminating discussions. I am also grateful to collaborators Dr Stephen Jenkins and Dr Marco Sacchi for complementing my experimental work with their DFT calculations.

I wish to thank all previous and current students of the Surface Physics Group, in particular David Ward and Dr Andrew Alderwick for generously sharing their computer knowledge and Dr Pepijn Kole and David Chisnall for lending me a hand in the lab on numerous occasions in the process of constructing MiniScat and the source. Together with Dr Fay Tuddenham, Suzanne Paterson, Eliza McIntosh, Kelsey Stoerzinger and Jianding Zhu they made our lab an enjoyable and exciting place to work at. I am also thankful to the members of the Cavendish workshops, in particular Nigel Palfrey, Rik Balsod, Gavin Ross and Peter Norman, for their help.

My special thanks go to Gonville \& Caius College, its graduate community and Caius Boat Club for giving me a life outside the lab.

I am extremely grateful to my family and Florian for their support and encouragement throughout my studies.

Finally, I would like to thank the Austrian Academy of Sciences, the Austrian Exchange Service and the EPSRC for financial support. 


\section{Publications related to the thesis}

- Results from Chapter 5 have been published in:

H. Hedgeland, B. A. J. Lechner, F. E. Tuddenham, A. P. Jardine, W. Allison, J. Ellis, M. Saachi, S. J. Jenkins and B. J. Hinch, Weak Intermolecular Interactions in an Ionically Bound Molecular Adsorbate: Cyclopentadienyl/ $\mathrm{Cu}(111)$, Phys. Rev. Lett., 106, 186101 (2011).

- Portions of the analytical models discussed in Chapter 2 have been published in:

F. E. Tuddenham, H. Hedgeland, A. P. Jardine, B. A. J. Lechner, B. J. Hinch and W. Allison, Lineshapes in quasi-elastic scattering from species hopping between non-equivalent surface sites, Surf. Sci., 604, 1459-1475 (2010).

- Results from Chapter 6 are in preparation for publication:

B. A. J. Lechner, H. Hedgeland, J. Ellis, W. Allison, M. Sacchi, S. J. Jenkins and B. J. Hinch, Quantum Influences in the Diffusive Motion of Pyrrole/Cu(111).

- The new nozzle design presented in Chapter 4 is in preparation for publication:

B. A. J. Lechner, H. Hedgeland, W. Allison, J. Ellis, and A. P. Jardine, A new design for a low-temperature high-intensity helium beam source.

- Results from Chapters 5, 6 and 7 have been presented at several conferences:

17th Interdisciplinary Surface Science Conference (ISSC-17), Reading, UK, 2009 (poster).

26th European Conference on Surface Science (ECOSS26), Parma, Italy, 2009 (poster).

18th International Vacuum Congress (IVC-18), Beijing, China, 2010 (talk).

18th Interdisciplinary Surface Science Conference (ISSC-18), Warwick, UK, 2011 (poster).

28th European Conference on Surface Science (ECOSS28), Wrocław, Poland 2011 (talk).

Gordon Research Conference on the Dynamics at Surfaces, Newport, USA, 2011 (poster and talk). 


\section{Contents}

1 Motivation 1

2 The helium-3 spin-echo experiment 5

2.1 Helium atom scattering . . . . . . . . . . . . . . 5

2.1.1 Diffraction measurements . . . . . . . . . . . . 6

2.1.2 Adsorption and desorption studies . . . . . . . . . . . . . . 7

2.1.3 Measuring surface dynamics . . . . . . . . . . . . . . . . . 9 9

2.2 Helium-3 spin-echo spectroscopy . . . . . . . . . . . . . . . . . . 11

2.3 The Cambridge helium-3 spin-echo spectrometer . . . . . . . . . . . . . . 12

2.4 Interpreting helium-3 spin-echo data . . . . . . . . . . . . . . . . 17

2.4 .1 Jump diffusion . . . . . . . . . . . . . . . . . . . . 20

2.4.2 Lateral interactions: De Gennes narrowing . . . . . . . . . . . . 32

2.4.3 Molecular dynamics simulations . . . . . . . . . . . . . . 32

3 A new helium atom scattering apparatus $\quad 35$

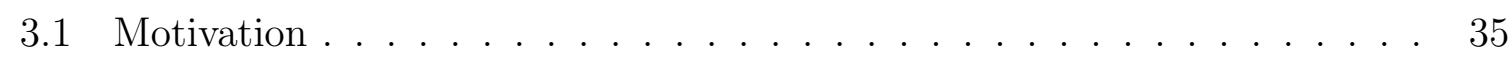

3.2 Design and experimental setup . . . . . . . . . . . . . 37

3.2.1 Vacuum system . . . . . . . . . . . . . . . . . 39

3.2.2 Power distribution and computer control . . . . . . . . . . . 43

3.2 .3 Beam source . . . . . . . . . . . . . . . . . . . 43

3.2.4 Scattering chamber and sample preparation facilities . . . . . . . 44

3.2.5 Detector . . . . . . . . . . . . . . . . 46

3.2.6 Two different instrument configurations . . . . . . . . . . . . . 47

3.3 Characterisation . . . . . . . . . . . . . . . . . 47

3.3.1 Uptake measurements . . . . . . . . . . . . . . . . . . . 48

3.3.2 Diffraction studies . . . . . . . . . . . . . . . . . . . 49

3.3.3 Thermal desorption spectroscopy . . . . . . . . . . . . . 51

3.4 Conclusions . . . . . . . . . . . . . . . . . . . . . . 53 
4 An improved high intensity supersonic helium beam source $\quad 55$

4.1 Introduction . . . . . . . . . . . . . . . . . 56

4.2 A new low temperature nozzle assembly . . . . . . . . . . . . 58

4.2 .1 Design . . . . . . . . . . . . . . . . . . . . . 59

4.2 .2 Characterisation . . . . . . . . . . . . . . 62

4.3 Effects of size and shape of the skimmer and its mount . . . . . . . . 67

4.3 .1 Design ......................... 68

4.3 .2 Performance . . . . . . . . . . . . . . 70

4.3.3 Discussion and future work . . . . . . . . . . . . . . . . 73

4.4 Conclusions . . . . . . . . . . . . . . . . . . . . . 75

5 The dynamics of cyclopentadienyl on $\mathrm{Cu}(111) \quad 77$

5.1 Literature background . . . . . . . . . . . . . . . . . . . . 78

5.1 .1 The structure of Cp . . . . . . . . . . . . . 78

5.1 .2 Cp adsorption on copper surfaces . . . . . . . . . . . . 79

5.1.3 Cp adsorption on other metal surfaces . . . . . . . . . . . . . . 79

5.1 .4 Metallocene adsorption . . . . . . . . . . . . . . . . 81

5.2 Experimental methods . . . . . . . . . . . . . . . . . . . . . 82

5.3 Adsorption behaviour . . . . . . . . . . . . . . . . 83

5.4 Measuring the surface dynamics . . . . . . . . . . . . . . 86

5.4.1 Multi-component lineshapes . . . . . . . . . . . . . . 86

5.4 .2 Jump diffusion curves . . . . . . . . . . . . . . . . . . . . . . . 90

5.4.3 An activation energy from temperature dependence . . . . . . . . 92

5.5 First principles density functional theory calculations . . . . . . . . . . . 94

5.6 Molecular dynamics simulations . . . . . . . . . . . . . . . . . 97

5.6.1 Creating a potential energy surface . . . . . . . . . . . . . 97

5.6.2 Modelling diffusion on a lattice of fcc and hcp sites . . . . . . . . 98

5.6.3 Microscopic aspects of the friction . . . . . . . . . . . . . . . 101

5.7 Weak lateral interactions . . . . . . . . . . . . . . . . . 104

5.8 Determining the energy difference between fcc and hcp sites . . . . . . 105

5.9 Intracell diffusion as a handle on friction and spring constant . . . . . . . 110

5.10 Conclusions . . . . . . . . . . . . . . . . . . . . . . 113

6 Quantum influences in the diffusive motion of pyrrole on $\mathrm{Cu}(111) \quad 115$

6.1 Literature background . . . . . . . . . . . . . . 116

6.1.1 The structure of pyrrole . . . . . . . . . . . . 116 
6.1.2 Pyrrole adsorption on copper surfaces . . . . . . . . . . . . . . 117

6.1.3 Pyrrole adsorption on other transition metal surfaces . . . . . . . 118

6.2 Experimental methods . . . . . . . . . . . . . . . . . . . . . . . 122

6.3 Adsorption behaviour . . . . . . . . . . . . . . . . . . . . . 123

6.4 Measuring the surface dynamics . . . . . . . . . . . . . . . . . . . . 128

6.4.1 Experimental lineshapes . . . . . . . . . . . . . . . . . . . 128

6.4.2 Hopping over an energy barrier . . . . . . . . . . . . . . . . . 131

6.4.3 Momentum transfer and coverage dependence of the dynamics . . 133

6.5 First principles density functional theory calculations . . . . . . . . . . 139

6.6 Molecular dynamics simulations investigate the effect of lateral interactions 141

6.6.1 Creating a potential energy surface . . . . . . . . . . . . . . . . . 142

6.6.2 Modelling the diffusion of pyrrole $/ \mathrm{Cu}(111) \ldots . . . . . .144$

6.6 .3 Interaction potential . . . . . . . . . . . . . . . . . 149

6.6.4 Influences of lateral interactions on the lineshapes . . . . . . . . . 151

6.7 Lateral changes in the zero point energies of vibrational modes . . . . . . 154

6.8 Conclusions and outlook . . . . . . . . . . . . . . . . . . 155

7 The diffusive motion of thiophene on $\mathrm{Cu}(111) \quad 157$

7.1 Literature background . . . . . . . . . . . . . . . . . . . . . . . 158

7.1.1 The structure of thiophene . . . . . . . . . . . . . . 158

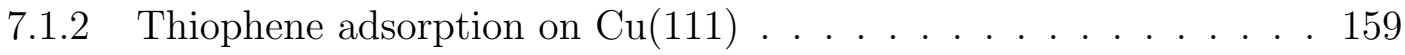

7.2 Experimental methods . . . . . . . . . . . . . . . . . 163

7.3 Adsorption behaviour . . . . . . . . . . . . . . . . . . . . . 164

7.4 Measuring the surface dynamics . . . . . . . . . . . . . . . . . . . 168

7.4.1 Complex lineshapes . . . . . . . . . . . . . . . . . . 168

7.4.2 Two competing activated processes . . . . . . . . . . . 170

7.4 .3 The diffusive motion . . . . . . . . . . . . . . . . . . . . 173

7.5 First principles density functional theory calculations . . . . . . . . . . 178

7.6 Molecular dynamics simulations reveal an exceptionally high friction . . . 179

7.7 Conclusions . . . . . . . . . . . . . . . . . . . . . . . . . . . . . 184

8 Conclusions $\quad 185$

8.1 Equipment development . . . . . . . . . . . . . . . . . 185

8.2 Dynamics experiments . . . . . . . . . . . . . . 186

$\begin{array}{lr}\text { Bibliography } & 189\end{array}$ 


\section{Chapter 1}

\section{Motivation}

Dynamical phenomena at the atomic scale influence and control a wide range of processes occuring on surfaces of solids [1]. A fundamental understanding of the forces guiding surface motion is thus of utmost importance for the advancement of many fields of science and technology. In its aim to increase information processing speed, the microelectronics industry is pushing manufacturing processes to produce ever smaller devices and is rapidly approaching the atomic length scale limit [2]. Therefore, new methods for the fabrication of electronic devices are being developed which use the forces between individual particles to control structures forming on surfaces. During the creation of such self-organised structures or regular self-assembled networks, adsorbates diffuse on the surface and adjust their orientation to form energetically stable superstructures. The final assembly is the result of a delicate balance between supramolecular chemistry i.e. inter-adsorbate interactions such as dipole-dipole, hydrogen bonding and van der Waals forces - and adsorbate-surface interactions. Studying diffusive processes promises a greater understanding of the forces driving self-assembly. Similarly, surface dynamics play a crucial role in heterogeneous catalysis where a change in the reaction rate occurs through adsorption of liquid or gaseous reactants on a solid [3]. Key requirements for a catalyst are the mobility of the adsorbed reactants and the release of the reaction product, which is controlled by lateral interactions and the forces bonding the adsorbates to the substrate.

Surface dynamic processes can be divided into two main categories: surface diffusion and vibrational motion $[1,4,5]$. Adsorbate motion occurs on a vast range of lengthand timescales, spanning many orders of magnitude. A wide variety of measurement techniques for the study of surface motion exists, each covering a different range $[5,6]$. Figure 1.1 summarises the principal experimental techniques which can be used to 
investigate surface diffusion and illustrates their respective application range. The techniques can be divided into three main groups which measure on different timescales. Optical techniques such as laser optical diffraction (LOD), second harmonic diffraction (SHD), fluorescence correlation spectrocopy (FCS), laser induced thermal desorption (LITD) and photoemission electron microscopy (PEEM) can measure on intermediate to large lengthscales. Meanwhile microscopic techniques such as scanning tunnelling microscopy (STM), field ion microscopy (FIM) and field emission microscopy (FEM), as well as the scattering techniques, quasi-elastic neutron scattering (QENS) and quasi-elastic helium atom scattering (QHAS), provide measurements on the atomic scale. The techniques probing atomic scale diffusion cover a vast range of different timescales. Microscopic imaging techniques measure diffusion by taking consecutive snapshots of the surface and are thus limited by the time required to record each image, which is on the order of milliseconds at best. Most vibrational and diffusive processes typically happen on much faster timescales at ambient temperatures $[1,4]$. To make the motion accessible to STM, samples therefore often need to be cooled to temperatures as low as $5 \mathrm{~K}$. Yet, many forms of surface dynamics only become apparent at elevated temperatures where atoms and molecules move on fast timescales, and cannot be investigated with microscopic techniques. Instead, scattering techniques can be used. QENS is only of limited use for the study of surfaces as neutrons penetrate the bulk, and can thus only be used in exceptional high surface area cases, e.g. for adsorbate diffusion on exfoliated graphite $[7,8]$. Helium atoms, on the other hand, scatter from the electron density of the surface and are sensitive to the surface only. QHAS, of which helium-3 spin-echo (HeSE) is a subset, can measure adsorbate motion on Ångström length- and pico- to nanosecond timescales, and is thus uniquely suited to measure adsorbate diffusion and vibrations, with no other currently available technique able to probe motion in the same regime $[9,10]$.

Since QHAS is a scattering experiment, it provides results in momentum transfer space, making the analysis of experimental data an involved and time consuming process. Until recently, QHAS in general and HeSE in particular have therefore been used primarily to study atomic or small molecular adsorbate systems, which could be interpreted using analytical models describing simple diffusion processes $[6,11]$. The aim of the present thesis is to extend the scope of application of HeSE to the diffusion of more complex adsorbates such as aromatic molecules, motivated by their similarity to technologically relevant organic materials [12]. One of the main challenges in investigating more complex surface dynamical systems is to determine the extent to which existing models can be applied, and to adapt them in an appropriate fashion. 


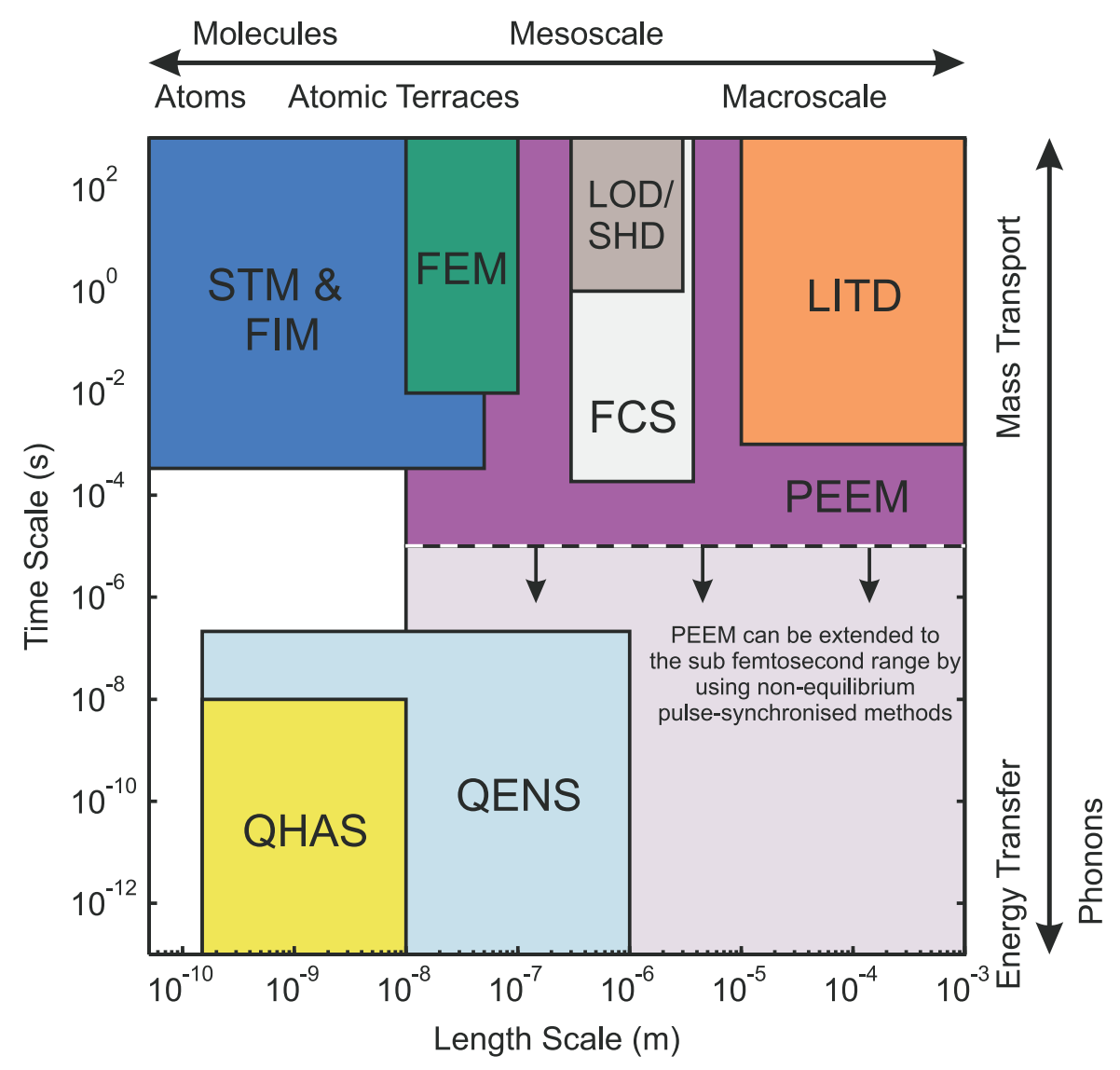

Figure 1.1: Schematic illustration of common measurement techniques probing surface motion on different length- and timescales (adapted from [6]). Optical techniques such as laser optical diffraction (LOD), second harmonic diffraction (SHD), fluorescence correlation spectrocopy (FCS), laser induced thermal desorption (LITD) and photoemission electron microscopy (PEEM) can measure surface diffusion on intermediate to large lengthscales, while microscopic techniques such as scanning tunnelling microscopy (STM), field ion microscopy (FIM) and field emission microscopy (FEM), as well as the scattering techniques quasi-elastic neutron scattering (QENS) and quasi-elastic helium atom scattering (QHAS) provide measurements on the atomic scale. QHAS is currently the only truly surface sensitive technique which can measure on atomic length- and pico- to nanosecond timescales.

Prior research of benzene on graphite has yielded exciting new information about the motion of molecular adsorbates [8]. In this system, the benzene exhibits an exceptionally large friction coefficient and performs Brownian motion on the surface, phenomena which have not been observed in simpler systems. In the present thesis, the dynamics of the diffusive motion of three five-membered aromatic molecules, cyclopentadienyl (Cp), pyrrole and thiophene, on a hexagonal copper surface are investigated using HeSE. The 
choice of a symmetry mismatch - fivefold molecules on a sixfold substrate - was made in an effort to minimise activation barriers for translational as well as rotational motion.

Using the example of $\mathrm{Cp}$, pyrrole and thiophene, this thesis demonstrates the power of comparative studies when the complexity of each individual system does not allow full modelling of the diffusion. As described in Chapters 5, 6 and 7, all three adsorbates move in hops between preferred adsorption sites - in contrast to the Brownian motion observed for benzene - and experience high friction similar to that found for benzene/graphite. Despite their extended nature, relatively simple centre-of-mass models provide a full understanding of the motion. While geometrically similar, $\mathrm{Cp}$, pyrrole and thiophene vary in their chemical properties due to the replacement of one of the $\mathrm{CH}$ groups of $\mathrm{Cp}$ with an NH group in the case of pyrrole, and a sulfur atom for thiophene. Indeed, the three molecules show distinct adsorption behaviour; each molecule prefers a different adsorption site on $\mathrm{Cu}(111)$. Furthermore, the adsorbate-substrate bond strengths range from chemisorption through ionic bonding for $\mathrm{Cp}$ to weak physisorption through van der Waals and weak $\pi$-bonding for pyrrole.

The investigation of more complex systems necessitates improvements to the experimental equipment, which is another focus of this thesis. Firstly, larger adsorbates tend to move more slowly than atoms, thus requiring improved machine resolution. A new helium beam source, which improves the resolution of the Cambridge HeSE spectrometer by a factor of 5.5 , is described in Chapter 4 . Secondly, as more extensive preliminary experiments are required prior to dynamics measurements of complex adsorbate systems, as well as further investigation of the adsorption behaviour, a new helium atom scattering apparatus has been developed (cf. Chapter 3), providing a facility to perform thermal desorption spectroscopy, diffraction experiments and studies of the degree of order on the surface during the adsorption process, thus enabling higher experimental throughput. 


\section{Chapter 2}

\section{The helium-3 spin-echo experiment}

Helium-3 spin-echo (HeSE) is a novel technique uniquely sensitive to adsorbate diffusion on atomic length- and pico- to nanosecond timescales $[6,13]$. This chapter introduces the principles underlying the experiment and presents typical data analysis methods. First, the helium atom scattering (HAS) technique is presented, with a general description of its use for structural studies, as well as dynamics measurements using quasi-elastic helium atom scattering (QHAS). Subsequently, the spin-echo principle is described and the Cambridge spectrometer presented. The final section introduces standard data analysis techniques to set the scene for the following chapters.

\subsection{Helium atom scattering}

In a HAS experiment a thermal beam of helium atoms is scattered from a crystal surface inside an ultra high vacuum (UHV) chamber and the intensity of the scattered beam is measured by a detector [11]. The use of helium atoms makes the technique exceptionally surface sensitive as they interact with the outermost surface electrons and do not penetrate the bulk. Furthermore, the low beam energies involved (typically between $5 \mathrm{meV}$ and $100 \mathrm{meV}$ ) and the inertness of helium imply that HAS is a non-destructive technique, permitting the investigation of biological samples that would degrade in a more energetic beam or when using charged probes, for example during low energy electron diffraction (LEED) experiments.

The principle of atom scattering is illustrated in Figure 2.1. Like other scattering techniques, HAS provides experimental results in momentum transfer space [14]. The lefthand side of Figure 2.1 defines the conventional nomenclature for the incident and outgoing 
wavevectors, $\boldsymbol{k}_{i}$ and $\boldsymbol{k}_{f}$. Specular reflection occurs when the incident scattering angle, $\Theta_{i}$, is equal to the outgoing angle, $\Theta_{f}$ and the total scattering angle of an experiment is defined as $\Theta=\Theta_{i}+\Theta_{f}$. The momentum transfer parallel to the surface, $\Delta \boldsymbol{K}=\boldsymbol{K}_{f}-\boldsymbol{K}_{i}$, can be calculated from the scattering geometry as $\Delta \boldsymbol{K}=k_{i} \sin \Theta_{i}-k_{f} \sin \Theta_{f}$. The right-hand part of Figure 2.1 illustrates scattering from an isolated adsorbate particle, shown in red. Due to their interaction with the outermost electrons of a surface species, helium atoms are extraordinarily sensitive to individual defects and small species such as hydrogen atoms. The apparent cross section of an adsorbate in a helium beam is typically much larger than the actual size of the atom or molecule [15].

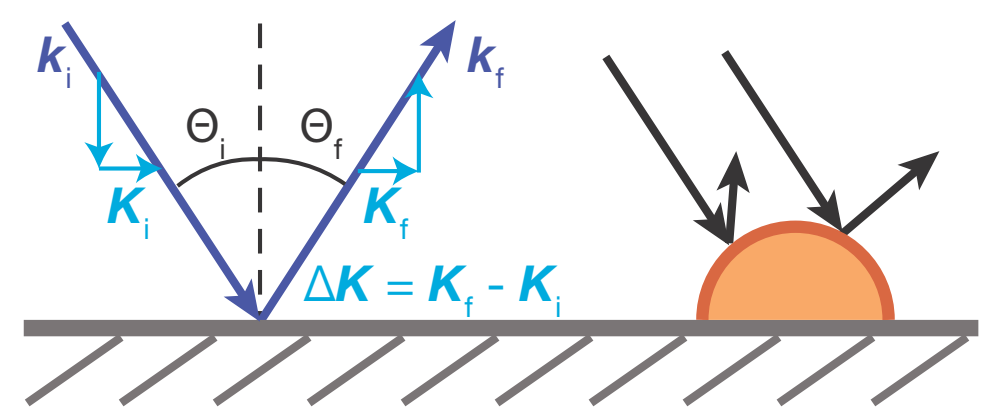

Figure 2.1: Schematic illustration of a helium atom scattering experiment. The wavevectors are denoted as $\boldsymbol{k}_{\boldsymbol{i}}$ for the incident and $\boldsymbol{k}_{\boldsymbol{f}}$ for the outgoing beam. By convention, the components parallel to the surface are given in capital letters, $\boldsymbol{K}_{\boldsymbol{i}}$ and $\boldsymbol{K}_{\boldsymbol{f}}$, and the momentum transfer parallel to the surface is $\Delta \boldsymbol{K}=\boldsymbol{K}_{\boldsymbol{f}}-\boldsymbol{K}_{\boldsymbol{i}}$. The incident and outgoing scattering angles are written as $\Theta_{i}$ and $\Theta_{f}$, respectively. The total scattering angle is $\Theta=\Theta_{i}+\Theta_{f}$. The right-hand side diagram illustrates scattering from an adsorbate, where the red line indicates the area around the adsorbate that the helium atoms scatter from. The scattering cross section of an adsorbate is typically much larger than its size.

\subsubsection{Diffraction measurements}

The helium beam has a wavelength comparable with atomic spacings and therefore a diffraction pattern is generated during scattering which contains information about the surface structure [11]. Each substrate atom or adsorbate can be considered as a point scatterer in the helium beam. The kinematic scattering approximation defines the scattered amplitude as the product of a form factor, $F$, describing scattering from each individual scatterer, and an amplitude structure factor, $S_{a}$, which contains information about the distribution of all scatterers on the surface and is coverage-dependent [6]. The 
total scattered amplitude at a certain position in momentum transfer is given as

$$
\Phi_{\text {total }}(\Delta \boldsymbol{K})=\sum_{j} F_{j}(\Delta \boldsymbol{K}) \cdot \exp \left(-i \Delta \boldsymbol{K} \cdot \boldsymbol{R}_{j}\right)
$$

where $F_{j}$ is the form factor and $\boldsymbol{R}_{j}$ the position of the $j^{\text {th }}$ scatterer. If the form factor for all scatterers is the same, i.e. all scattering centres are identical, the equation can be simplified to

$$
\Phi_{\text {total }}(\Delta \boldsymbol{K})=F(\Delta \boldsymbol{K}) \cdot \sum_{j} \exp \left(-i \Delta \boldsymbol{K} \cdot \boldsymbol{R}_{j}\right)=F(\Delta \boldsymbol{K}) \cdot S_{a}(\Delta \boldsymbol{K})
$$

The scattered intensity is obtained from $I=|F(\Delta \boldsymbol{K})|^{2} \cdot\left|S_{a}(\Delta \boldsymbol{K})\right|^{2}$. Helium atoms have a de Broglie wavelength on the order of Ångströms, ensuring resolution of atomic scale features during a HAS experiment.

Helium atom scattering instruments can be divided into two main types, "fixed angle" machines, where the angle between incident and outgoing beam is fixed, and movable systems, where a rotating detector alters the total scattering angle, $\Theta$ [15]. All experiments presented in this thesis are from fixed angle instruments, the Cambridge HeSE spectrometer (cf. Section 2.3) and a separate atom scattering apparatus which is presented in Chapter 3. In a fixed angle scattering experiment, the incident and outgoing scattering angles are determined by the orientation of the crystal which can be rotated around the vertical centre axis of the scattering chamber. In contrast to diffraction experiments with rotating detector arms, where the incident scattering angle can be kept constant, turning the sample in a fixed angle machine always alters the incident and outgoing scattering angles at the same time. Diffraction measurements performed in a fixed angle scattering apparatus are therefore intrinsically asymmetric, even for a perfectly aligned crystal. The heights of equivalent diffraction peaks either side of specular are therefore different, which needs to be considered in data analysis and when comparing diffraction scans with results from different instruments or simulations.

\subsubsection{Adsorption and desorption studies}

In addition to diffraction experiments, measurements of the specular helium reflectivity can provide information about the degree of order on the surface [15]. The intensity of the specular beam is greater for a highly ordered surface as less signal is lost in other 
scattering directions. Measuring the proportion of incident helium that is scattered into the specular beam is a means of determining the quality of a substrate surface as it is reduced by step edges and defects. Equally, monitoring the specular helium reflectivity during a dosing or desorption process can provide information about the change in order on the surface.

A dosing process can be monitored by recording the specular helium reflectivity as a function of exposure. The resulting curve is generally termed an "uptake" curve. Adsorption onto a clean and flat substrate initially reduces the degree of order on the surface, resulting in lower reflectivity towards the helium beam. When regular structures are formed, the degree of order and thus the helium reflectivity increase. Uptake curves are useful for estimating the coverage at a given specular attenuation level, to study the lateral interactions between adsorbates and to determine where regular overlayer structures occur [15]. In the low coverage limit, the total scattering cross section, $\Sigma$, for isolated adsorbates can be calculated as

$$
\Sigma=-\left.\frac{1}{n_{s}} \cdot \frac{1}{I_{0}} \cdot \frac{d I}{d \Theta}\right|_{\Theta=0},
$$

where $\Theta$ is the coverage given by the number of adsorbates per substrate atom, $n_{s}$ is the number of substrate atoms per unit area, and $I / I_{0}$ is the specular helium beam attenuation at coverage $\Theta$. If one can assume non-interacting adsorbates occupying random adsorption sites with large cross sections that overlap, then the formula also holds at higher coverages,

$$
\Sigma=-\frac{1}{n_{s}} \cdot \frac{1}{I_{0}} \cdot \frac{d I}{d \Theta}
$$

hence

$$
\frac{I}{I_{0}}=\exp \left(-\Theta \Sigma n_{s}\right)
$$

Equation 2.5 implies that the specular attenuation as a function of coverage is linear on a logarithmic scale, in the simplest case. The scattering cross sections of repelling adsorbate particles overlap less, increasing the total cross section seen by the helium beam compared to that for non-interacting adsorbates and thus the uptake curve falls below the linear line. When attractive interactions influence the distribution of adsorbate particles, on the other hand, the curve rises above linear. 
In a similar way, a desorption process can be monitored with specular helium reflectivity. In the case of molecular desorption, reversal of the uptake curve is observed, while disordering or decomposition of adsorbates typically results in a drop in the observed reflectivity.

\subsubsection{Measuring surface dynamics}

In addition to structural studies, HAS can be used to investigate surface dynamics by measuring the difference in energy distribution in the beam before and after scattering from the sample $[10,11]$. The technique is termed quasi-elastic helium atom scattering (QHAS).

In a QHAS experiment, the energy spectrum of the scattered helium beam is determined $[10,11]$. If the surface remains static during the scattering event, the helium atoms are scattered elastically, resulting in a peak in the energy domain with a finite width determined by the velocity spread in the beam and properties of each individual instrument. In case of non-periodically moving adsorbates, however, the elastic peak is broadened by a quantum mechanical form of Doppler broadening - hence the name "quasi-elastic". In addition, the helium beam can excite or de-excite surface phonons, resulting in an inelastic peak in the energy spectrum, where the energy difference between elastic and inelastic peak is defined by the energy loss or gain during the scattering process [16]. A QHAS experiment is the surface sensitive analogue of quasi-elastic neutron scattering which measures diffusion in bulk materials [7]. Therefore, the existing theoretical framework for the neutron scattering experiments could be adopted for the QHAS technique.

Theoretical foundations are in Van Hove's scattering theory describing motion by the Van Hove pair correlation function $G(\boldsymbol{R}, t)$ which defines the probability of finding an atom at position $\boldsymbol{R}$ at the time $t$ given the presence of an atom (the identical or a different one) at the origin at $t=0$ [17]. It is possible to divide $G(\boldsymbol{R}, t)$ into

$$
G(\boldsymbol{R}, t)=G_{S}(\boldsymbol{R}, t)+G_{D}(\boldsymbol{R}, t)
$$

where $G_{S}(\boldsymbol{R}, t)$ is the self-correlation function, describing the probability of finding the same atom at $\boldsymbol{R}$ and $t$ given it was at the origin at $t=0$, and $G_{D}(\boldsymbol{R}, t)$ the distinct correlation function relating the position of two distinct atoms. Vineyard showed that $G_{D}(\boldsymbol{R}, t)$ can be written in terms of $G_{S}(\boldsymbol{R}, t)$ for non-interacting particles - which is 
typically true in the low coverage limit - and the Van Hove correlation function can be expressed in terms of the self-correlation function alone [18], facilitating the interpretation of experiments.

Within the kinematic scattering approximation, the probability of scattering into a given angle, $\partial \Omega$, and energy, $\partial \omega$, is related to the pair correlation function through

$$
\begin{aligned}
\frac{\partial^{2} \boldsymbol{R}}{\partial \Omega \partial \omega}(\Delta \boldsymbol{K}, \omega) & =n_{d}|F(\Delta \boldsymbol{K}, \omega)|^{2} \iint G(\boldsymbol{R}, t) \exp [i(\Delta \boldsymbol{K} \boldsymbol{R}-\omega t)] d \boldsymbol{R} d t \\
& =n_{d}|F(\Delta \boldsymbol{K}, \omega)|^{2} S(\Delta \boldsymbol{K}, \omega)
\end{aligned}
$$

where $n_{d}$ is the density of adsorbates on the surface [17]. The dynamical structure factor (DSF), $S(\Delta \boldsymbol{K}, \omega)$, is an intensity structure factor and is the Fourier transform of the correlation function in space and time. The Fourier transform of the correlation function in space is called the intermediate scattering function (ISF) $I(\Delta \boldsymbol{K}, t)$, which is related to the DSF by a temporal Fourier transform:

$$
G(\boldsymbol{R}, t) \stackrel{F T}{\Longleftrightarrow} I(\Delta \boldsymbol{K}, t) \stackrel{F T}{\Longleftrightarrow} S(\Delta \boldsymbol{K}, \omega) .
$$

The original QHAS experiments were carried out with a time-of-flight (TOF) detection system, where a chopped beam is scattered from the surface and its velocity after the scattering event is measured [19]. Any changes in velocity can be related to dynamical processes on the surface during the scattering process. TOF experiments provide a measure of the DSF, giving an inelastic peak for phonon excitations and an energy broadening, $\Delta E$, of the elastic peak for scattering from aperiodically diffusing adsorbates. Measurements of the dependence of $\Delta E$ on the momentum transfer parallel to the surface, $\Delta \boldsymbol{K}$, provide information about the diffusion process.

The main limitation of the QHAS-TOF technique is its requirement for high energy resolution so that the broadening of the elastic peak can be determined. The elastic energy peak is convoluted with experimental factors such as the energy distribution in the incident helium beam and the chopper time resolution, limiting the energy resolution to approximately $0.3 \mathrm{meV}$ [11]. Therefore, the time-of-flight technique can only be applied to exceptionally fast moving systems, putting it at the lower end of the timescale in Figure 1.1. HeSE, on the other hand, provides a direct measure of the energy broadening, allowing for the investigation of diffusion on pico- to nanosecond timescales, as explained in the following section. 


\subsection{Helium-3 spin-echo spectroscopy}

While QHAS-TOF operates in the energy domain, HeSE instead investigates the time domain, providing a measure of the ISF. Experimental results are therefore the temporal Fourier transform of TOF measurements. The key advantage of the HeSE technique is that it measures the energy change directly, rather than in relation to the energy of the helium beam, thus increasing the resolution of the experiment by two orders of magnitude over QHAS-TOF [11]. Measurements are typically analysed directly in the time domain to study aperiodic diffusion, or can be Fourier transformed into an energy spectrum for the investigation of periodic motion such as phonons.

The principle underlying helium-3 spin-echo is to use the nuclear spin of a helium-3 atom as an internal timer $[6,14,20,21]$, in analogy to the neutron spin-echo technique [22], all the while retaining the non-destructive nature and surface sensitivity of the HAS experiment. See Figure 2.2 for an illustration of the setup of a HeSE experiment. A supersonic helium beam source creates a beam of helium-3 atoms which is then polarised in a direction perpendicular to the beam axis by the combination of a hexapole magnet focussing one spin polarisation (defocussing the other) and a dipole magnet that aligns all the spins in the same direction. A variable magnetic field parallel to the beam direction is created in a solenoid, splitting the helium wavepacket into two spin states, parallel and antiparallel to the field. The field separates the components in both space and time. The state parallel to the field is accelerated as it enters the solenoid and the state antiparallel is decelerated, so that they reach the sample a time period, $t$, apart, which is called the spin-echo time. $t$ depends on the mass of helium-3, $m$, its gyromagnetic ratio, $\gamma$, the mean velocity of the beam, $v_{0}$, and the magnetic field integral along the path of the beam $[6]$ :

$$
t=\frac{\hbar \gamma}{m v_{0}^{3}} \int B d l
$$

After scattering from the sample surface the two components are recombined in an identical but reversed magnetic field. The scattered beam is then spin-analysed by another combination of dipole and hexapole magnets to provide a polarisation measurement as it enters the detector. If the surface remains static during the scattering process the helium atoms scatter elastically, the two spin components are recombined and the entire scattered beam passes through the analyser into the detector. If, however, the atoms or molecules on the surface move during $t$, the two components do not scatter identically 
and therefore do not recombine to give the same final spin-state, resulting in a reduction in net polarisation reaching the detector. HeSE thus measures the coherence of the two spin components after scattering from the surface, which typically decays with time.

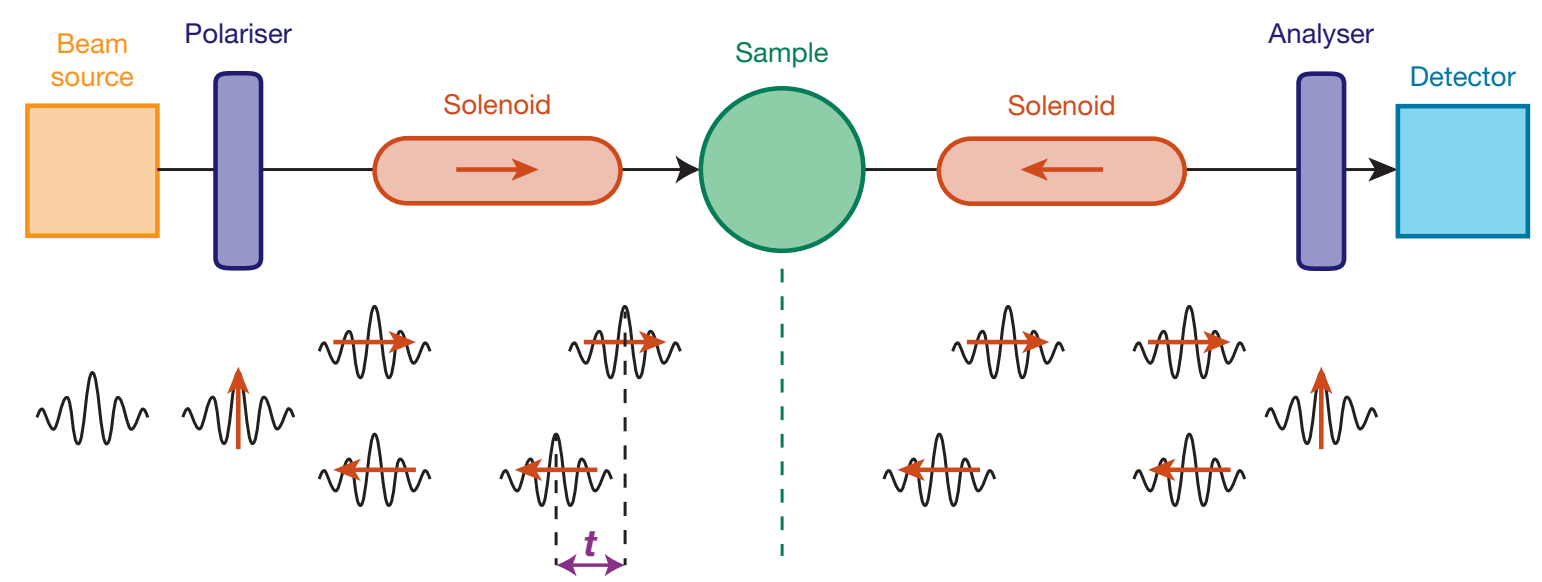

Figure 2.2: Schematic overview of the helium-3 spin-echo experiment. The different components of the instrument are outlined in the top row, while the spin state of the wavepackets of the helium-3 beam during the experiment is illustrated below. The first solenoid field splits the helium-3 atom into two wavepackets which reach the sample separated by a time, $t$. A second solenoid recombines the two components. If the two wavepackets do not recombine to give the same final spin state, a reduction in polarisation is measured in the detector.

\subsection{The Cambridge helium-3 spin-echo spectrometer}

After a prototype was presented by De Kieviet and coworkers [20,21], the Surface Physics group at Cambridge developed the first HeSE apparatus with sufficient momentum transfer space resolution to map out adsorbate diffusion, where accessible timescales range from $0.01 \mathrm{ps}$ to $680 \mathrm{ps}[13,23]$. Figure 2.3 shows a photograph of the apparatus, with annotations illustrating the location of the main components, in analogy to the diagram in Figure 2.2.

The instrument is of the fixed angle type, with a total scattering angle of $44.4^{\circ}$, an angular resolution of $0.1^{\circ}$ and an energy resolution of $3 \mu \mathrm{eV}$ (or an ultimate resolution of $20 \mathrm{neV}$, corresponding to a $1 \%$ decay in polarisation at the maximum spin-echo time) [23]. The helium beam is created in a supersonic helium beam source, which is described in detail in Chapter 4. A recycling system filters and compresses the 


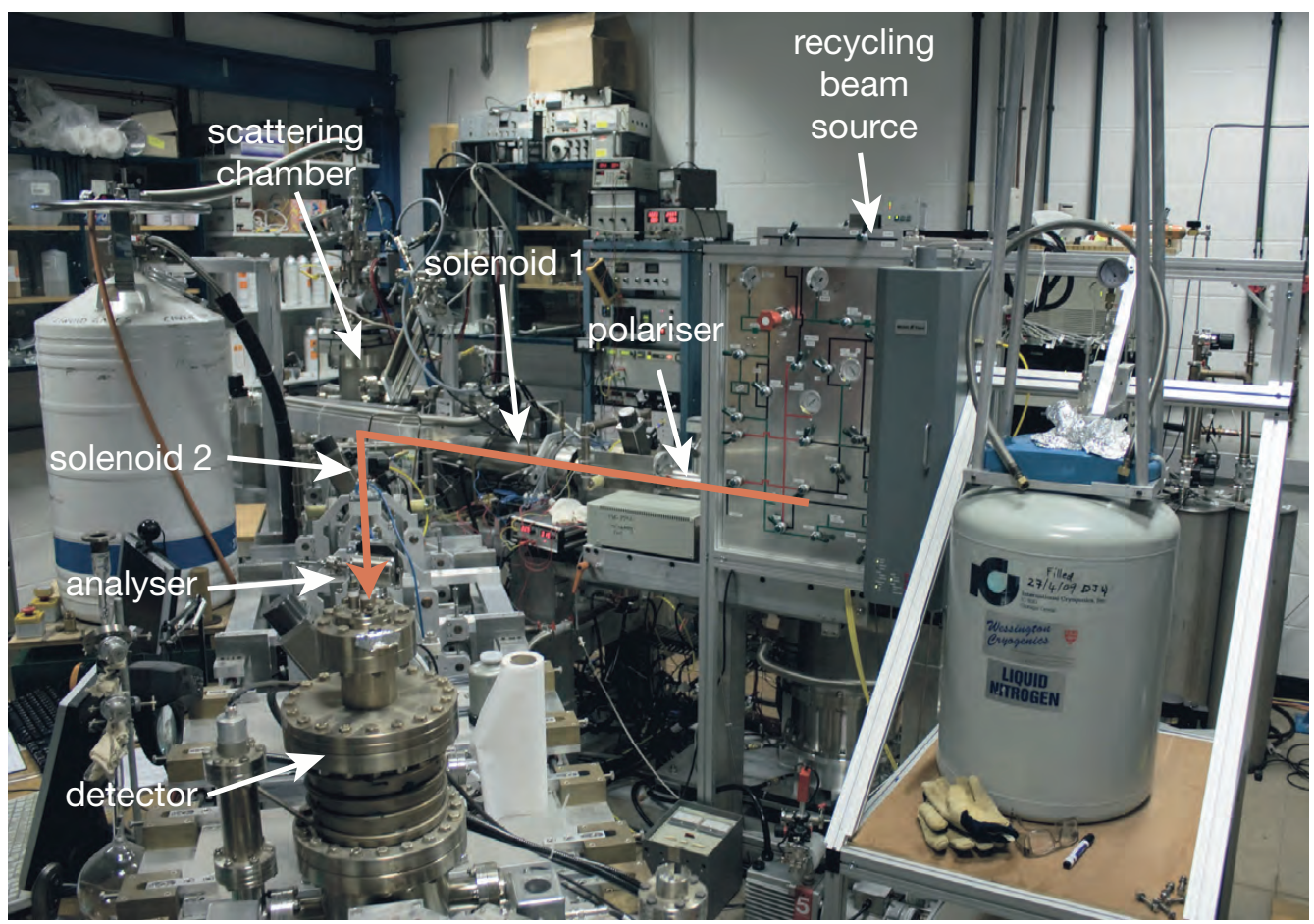

Figure 2.3: Photograph of the Cambridge HeSE spectrometer. Annotations highlight its main components, while a red arrow illustrates the path of the helium beam during an experiment.

helium-3 gas which is then expanded into the source chamber through a cooled nozzle and recirculated back into the system after expansion [23-25]. The nominal beam energy for the spectrometer is $8 \mathrm{meV}$, for which energy the magnets provide optimal focussing. Two combinations of permanent hexapole/dipole magnets spin-polarise and spin-analyse the helium-3 beam $[23,26]$, while a variable magnetic field in two solenoids splits and recombines the wavepackets. The magnetic field can be controlled by the current running through the two solenoids and defines the spin-echo time, $t$. A pair of coils mounted on the sample manipulator rotates the plane of spin polarisation when the helium atoms change direction during the scattering process.

The sample is located in the scattering chamber, mounted on a six-axis manipulator. Horizontal $\mathrm{x} / \mathrm{y}$-motion and vertical z-motion allows positioning of the sample in the location where the ingoing and outgoing beam arms meet, while rotation around the vertical centre axis ( $\gamma$-rotation) changes the scattering angles, $\Theta_{i}$ and $\Theta_{f}$. Tilting of the crystal with respect to the plane of the incident and outgoing beam enables alignment of the sample perpendicular to the scattering plane ( $\beta$-rotation). The sixth degree of freedom is rotation within the crystal plane to change the azimuthal direction ( $\alpha$-rotation). 
A new sample transfer manipulator, recently developed by Pepijn Kole, enables the exchange of samples without breaking the vacuum, thereby maintaining UHV conditions in the chamber [27]. Sample cooling is provided through a coldfinger which can be filled with liquid nitrogen or liquid helium through a transfer tube. Temperature control is achieved using a Eurotherm controller reading the measurements from a chromel/alumel type $\mathrm{K}$ thermocouple connected to the sample mount and controlling the temperature by counter-heating the cooled sample radiatively using a tungsten filament. The scattering chamber is equipped with a sputter gun for cleaning the sample by ion bombardment as well as facilities for dosing gaseous, liquid and solid substances, and a mass spectrometer for the identification of components in the background gas or dosed species.

Detection of the helium beam is achieved in a custom-built helium detector [23, 28]. The helium atoms are ionised by electrons from a hot tungsten filament and pass through ion extraction optics and a magnetic sector mass filter to select mass-3 ions before they are counted in an electron multiplier. After completion of the experiments presented in this thesis, David Chisnall has recently installed a new detector on the HeSE spectrometer which uses a solenoid magnetic field to confine electrons in the ionisation region $[29,30]$. The sensitivity of the new detector is improved by approximately three orders of magnitude over that of the previous HeSE detector, thus opening a completely new range of measurements.

The observable quantity in a HeSE experiment is the intensity, $I$, of the helium signal in the detector, which can be related to the polarisation, $P$, through

$$
P=\frac{I_{\max }-I_{\min }}{I_{\max }+I_{\min }}
$$

The polarisation, in turn, is directly proportional to the intermediate scattering function [6]. In order to obtain maximum signal intensity in the detector, the polarisation selected by the analyser hexapole magnet must be identical to the polarisation of the beam created in the polarising hexapole. The HeSE technique thus relies on the precise measurement of the spin polarisation of the helium-3 beam. Loss of polarisation can occur through a large velocity spread in the beam, so that beam energies other than the optimal $8 \mathrm{meV}$ target are present, or stray magnetic fields. To minimise stray fields, components close to the path of the helium beam are made from non-magnetic materials as well as magnetically shielded. Despite all these efforts, however, the polarisation of the beam changes over time to a certain extent, adding errors to the measurement. 
During a HeSE experiment, both the real and the imaginary components of the polarisation of the beam are measured, thus allowing Fourier transformation of the spectra without imposing artificial symmetry [31]. A phase coil creates a magnetic field which switches between the two components by adding an extra $\pi / 2$ to the incoming phase, thus rendering the two solenoid fields unequal. The phase coil can be used to correct for errors caused by stray fields by regular calibration to determine the currents required for obtaining either the real or the imaginary polarisation. Calibration is achieved at a position where the imaginary polarisation is known to be zero, such as at specular reflection.

Originally, a two-point measurement technique was used for HeSE experiments, measuring the real and imaginary polarisation for each point in the ISF by setting the phase coil current to the values determined during calibration. The drawback of this method is that it relies heavily on the calibration of the phase coil current, as illustrated in Figure 2.4, since any drifts in the polarisation are not accounted for. When looking at the two oscillating curves in the Figure, which are slightly offset from each other, it becomes apparent that the real component is not heavily dependent on ultra-precise calibration since the curve is fairly flat at this position and small errors in phase coil current thus do not change the signal intensity measured in the detector significantly. The imaginary component, however, is located on the steepest slope of the curve and the smallest change in location of the exact measurement point result in a large change in intensity. To avoid such errors, a new measurement technique was recently developed by David Ward [32]. Instead of attempting to measure the precise real and imaginary components, four points are measured which describe the central oscillation of the polarisation. The location of the real and imaginary component can then be determined for each individual measurement, lessening the importance of correct calibration. Furthermore, the four-point method allows the direct measurement of the polarisation rather than the intensity of the helium signal in the detector, eliminating errors due to detector and background drifts.

For all but the very fastest diffusive motion, the real part of the polarisation is significantly larger than the imaginary one [33,34]. Figure 2.5 shows a typical HeSE measurement of the real polarisation as a function of $t$, plotted in red, and the imaginary polarisation, presented in green. The real signal shows a large change in intensity across all timescales, while the imaginary component only exhibits an oscillatory feature at short times and drops to zero at times longer than $2 \mathrm{ps,} \mathrm{which} \mathrm{is} \mathrm{where} \mathrm{the} \mathrm{diffusive} \mathrm{motion}$ dominates. The imaginary polarisation can thus be neglected when adsorbate diffusion is studied, as is the case for the experiments presented in this thesis. In this regime, 


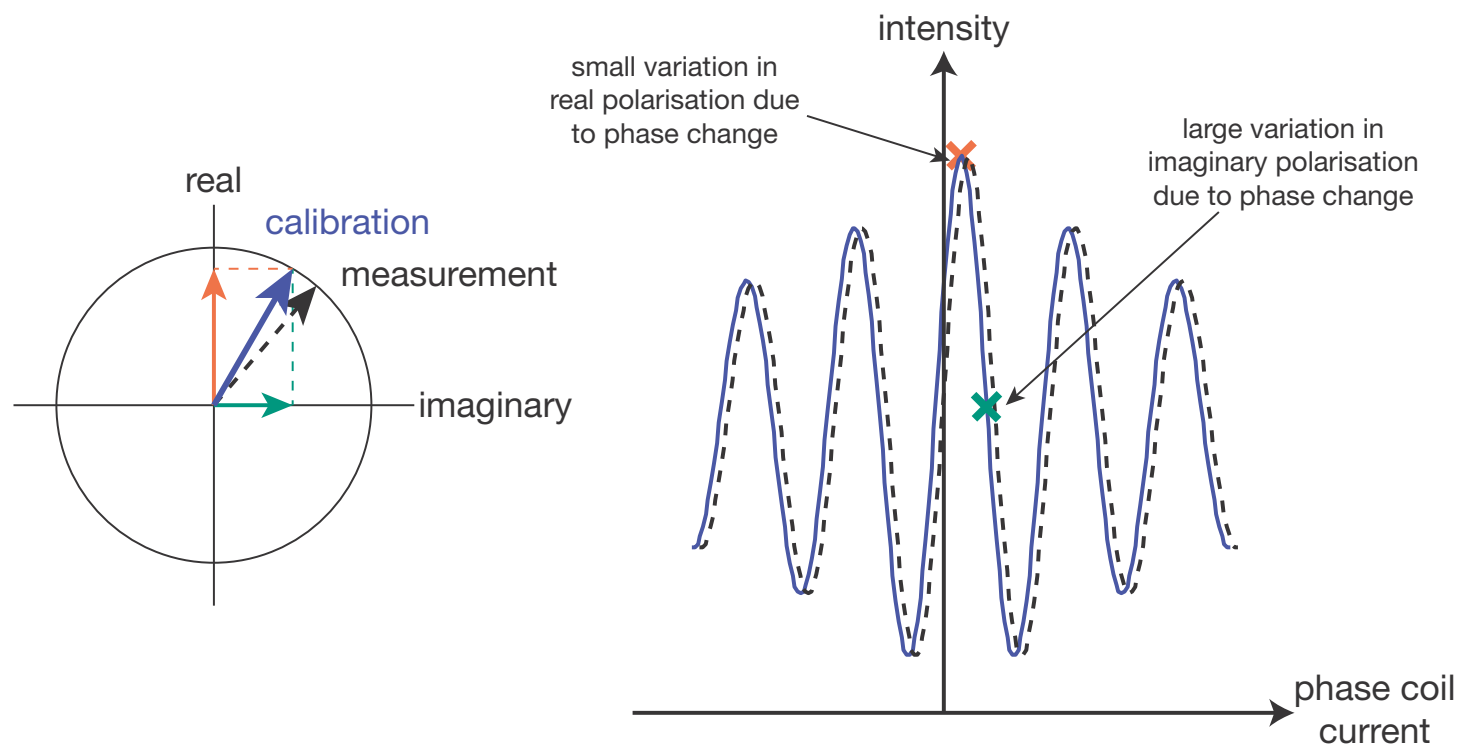

Figure 2.4: Schematic illustration of the real and imaginary polarisation measured in a HeSE experiment. By altering the current in the phase coil and thereby the magnetic field, the polarisation of the beam is changed periodically, as illustrated by a unit circle on the left, and the signal intensity measured in the detector varies sinusoidally, as shown on the right, dependent on the proportion of the beam allowed to pass through the analyser hexapole. The phase coil is calibrated by measuring the oscillation (solid blue line) at a point where the imaginary polarisation is known to be zero and recording the phase coil current giving the real (red) and imaginary (green) signal, which is then used to measure the real and imaginary polarisation during a dynamics measurement. If the polarisation changes in between the calibration and the dynamics measurement, as illustrated by the dashed line, the measured polarisation is wrong. The error in the real component is small as the curve is relatively flat at that point while the imaginary signal exhibits large variation.

the two- and four-point techniques show good agreement and both provide reproducible data. For measurements of phonons or other features on short timescales, however, the imaginary signal is of great importance and the four-point method is therefore much superior $[27,32]$.

The data presented in Chapters 5 and 6 of this thesis were recorded using the two-point method, while the data in Chapter 7 are from four-point measurements. This is reflected in the nomenclature used in figures showing HeSE measurements where "intensity" is used for two-point, and "polarisation" for four-point measurements. Since the main focus of the thesis is the analysis of inter-cell diffusion, using only the real polarisation, both methods are equally valid. Care must be taken, however, when features on faster 


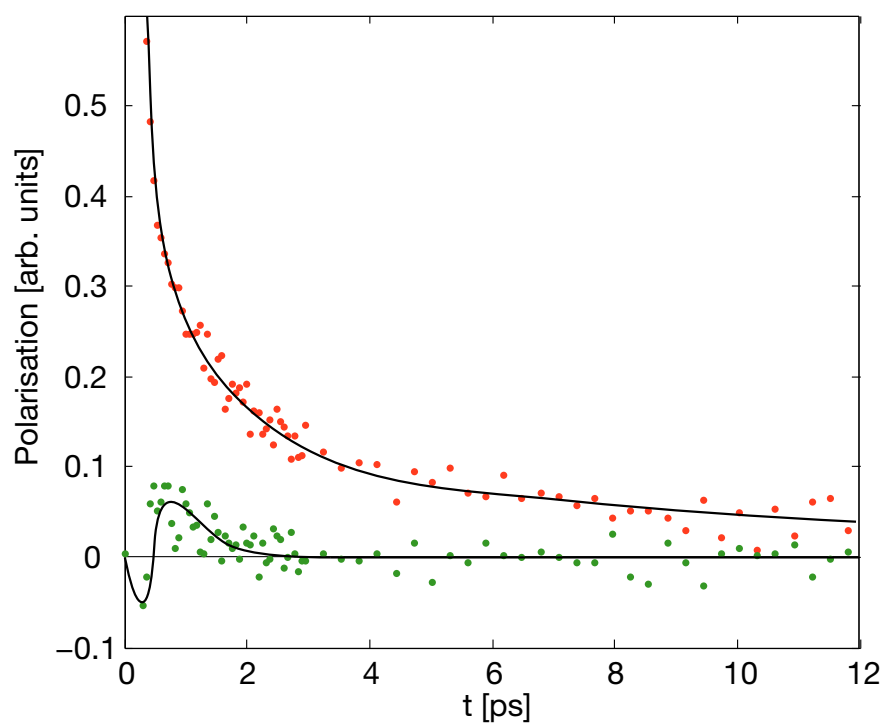

Figure 2.5: A typical HeSE measurement, showing the real and imaginary polarisation in red and green, respectively. While the real component shows a large change in signal, the imaginary contribution exhibits an oscillatory feature at very short timescales $(<2 \mathrm{ps})$ but is essentially zero at larger times, where diffusive motion dominates the signal.

timescales - not discussed here - are observed as they might be influenced by inaccuracies in the calibration.

\subsection{Interpreting helium-3 spin-echo data}

Since HeSE measures in momentum transfer space, data interpretation is not straightforward. A typical measurement of the intermediate scattering function is shown in Figure 2.6. On the linear time axis (left-hand side panel), details on short timescales are difficult to make out. For this reason HeSE measurements are often presented on a logarithmic scale, as illustrated in the right-hand side panel of the same figure. The two main features in this curve are oscillations at short times, $t$, and a global decay. Oscillations are caused by periodic motion on the surface, such as substrate phonons. To analyse such vibrational features, the spectra can be reconstructed to give the corresponding dynamical structure factor, which shows energy gain and loss peaks. The global decay, on the other hand, is a signature for aperiodic motion on the surface, such as diffusive motion of an adsorbate. To study the diffusion in more detail, the rate at which the signal decays ("dephasing rate"), $\alpha$, can be determined by fitting a function of the form 
$f(t)=a \cdot \exp (-\alpha t)+c$, where $a$ is the preexponential factor and $c$ the static component. Fitting is performed using the least-squares algorithm, as implemented in Matlab. This method iteratively changes variables $a, c$ and $\alpha$ to minimise the least-squares error between the measured data and the exponential function. All lines are checked by eye, to prevent misfits and to confirm that the line goes through the centre of the vibrations. $\alpha$ is proportional to the energy broadening of the elastic peak, $\Delta E$, measured in QHAS-TOF experiments. The position to which the decay levels off to is called "static component" as it is typically due to scattering from static defects on the surface. The intensity of the static component is a diffraction pattern and thus varies with momentum transfer.
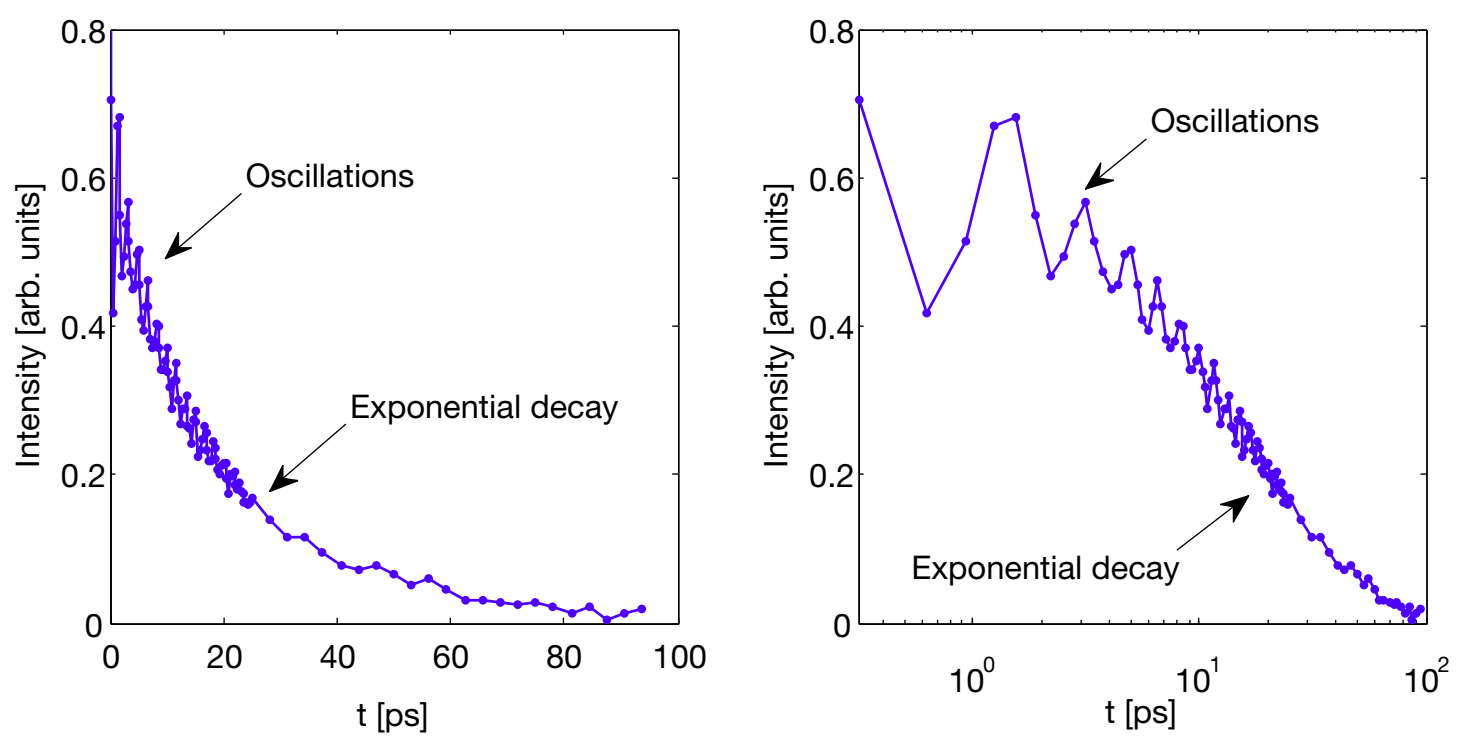

Figure 2.6: Illustration of a typical HeSE measurement exhibiting oscillatory features and a global decay. The same data are presented on a linear (left) and on a logarithmic (right) time axis to illustrate the advantage of a logarithmic presentation, allowing simultaneous investigation of features on a range of different timescales.

At coverages below a monolayer, many adsorbates diffuse on the substrate $[1,5]$. To study this two-dimensional diffusion, HeSE measurements are performed for a range of scattering angles and along (usually two) different high symmetry crystal directions. The variation of the dephasing rate with momentum transfer parallel to the surface, $\alpha(\Delta \boldsymbol{K})$, is dependent on the exact type of diffusion. Figure 2.7 illustrates the $\alpha(\Delta \boldsymbol{K})$ dependence for three different diffusion models [11].

Continuous Brownian motion gives exponentially decaying lineshapes in the ISF and a quadratic variation of the dephasing rate with $\Delta \boldsymbol{K}$, where the proportionality factor 


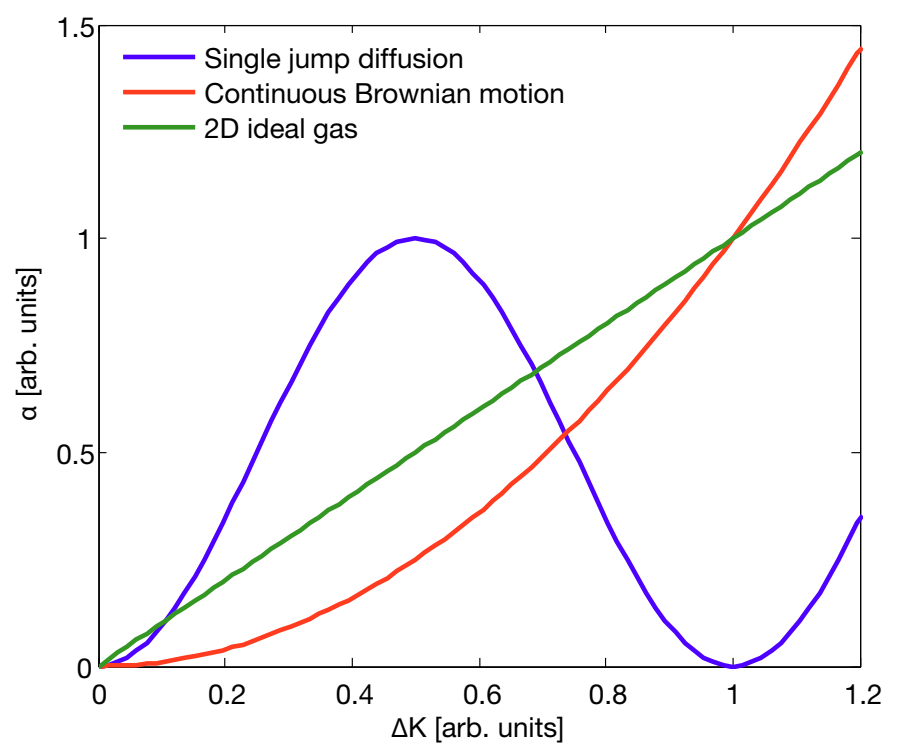

Figure 2.7: Different types of diffusion give a different dependence of the dephasing rate on the momentum transfer. The red line shows continuous Brownian motion, which exhibits a quadratic $\alpha(\Delta \boldsymbol{K})$ dependence. Ideal gas-like diffusion shows a linear relationship (green line), while discrete hops between preferred adsorption sites give a periodic variation with $\Delta \boldsymbol{K}$, as illustrated by the blue line.

between $\alpha$ and $\Delta \boldsymbol{K}^{2}$ is the diffusion coefficient, $D$ :

$$
\alpha=D \Delta \boldsymbol{K}^{2}
$$

Ideal gas-like diffusion, on the other hand, results in Gaussian lineshapes and a linear $\alpha(\Delta \boldsymbol{K})$ dependence,

$$
\alpha=\sqrt{\frac{2 \ln (2) k_{B} T}{m}} \Delta \boldsymbol{K}
$$

where the slope depends on the mass, $m$, of the diffusing adsorbate and the temperature, $T$, and $k_{B}$ is the Boltzmann constant.

The most common form of diffusion is in jumps between preferred adsorption sites, on a corrugated potential energy landscape $[5,11]$. In this case, the ISFs are typically of an exponentially decaying form and the $\alpha(\Delta \boldsymbol{K})$ dependence is a sum of sinusoids. The precise lineshapes and the variation of $\alpha$ with momentum transfer depend on the periodicity of the substrate lattice and the arrangement of the sites the adsorbate hops on, as described in the next section. 


\subsubsection{Jump diffusion}

The simplest form of jump diffusion occurs between sites forming a Bravais lattice, implying that the symmetry of the sites which the adsorbate occupies before and after the hop is the same. The Chudley \& Elliot model for hopping motion describes the ISF as

$$
I(\Delta \boldsymbol{K}, t)=\exp (-\alpha(\Delta \boldsymbol{K}) \cdot t)
$$

with the decay rate, $\alpha$, given by

$$
\alpha(\Delta \boldsymbol{K})=2 \sum_{k} \nu_{k} \sin ^{2}\left(\frac{\Delta \boldsymbol{K} \cdot \boldsymbol{l}_{k}}{2}\right)
$$

where $\nu_{k}$ is the jump frequency, which is the inverse of the residence time, $\tau_{k}$, in the adsorption site [35]. The jump vector $\boldsymbol{l}_{k}$ defines the direction and length of each possible hop, which depends on the substrate lattice constant.

The experiments presented in the present thesis show jump diffusion on a $\mathrm{Cu}(111)$ surface, schematically illustrated in Figure 2.8. Geometrically, three types of highsymmetry sites can be distinguished: top $(\mathrm{T})$, threefold hollow $(\mathrm{H})$ and twofold bridge (B) sites. Copper forms a face-centred cubic (fcc) crystal structure, implying that there are two different kinds of hollow sites on the (111) surface - an hcp site on top of an atom in the second layer, and an fcc site with no atom directly underneath - which can thus be energetically different in the interaction with an adsorbate.

Top sites form a simple Bravais lattice on $\mathrm{Cu}(111)$, as well as each type of hollow sites on its own, so that the Chudley \& Elliot model can be used to describe the hopping motion. The $\alpha(\Delta \boldsymbol{K})$ curves predicted by the analytical model for single jumps on such a Bravais lattice projected onto the $<1 \overline{1} 0>$ and $<11 \overline{2}>$ azimuths are presented in Figure 2.9, showing a simple sinusoidal form along $<11 \overline{2}>$ and a sum of two sinusoids along $<1 \overline{1} 0>$. Bridge sites, or both types of hollow sites together, however, form non-Bravais lattices. An adsorbate hopping on such a lattice changes the possible jump directions after each jump. In order to predict the lineshapes and the $\alpha(\Delta \boldsymbol{K})$ dependence for hopping on such lattices, an extended model is required. Models for three-dimensional jump diffusion on nonBravais lattices can be found in the neutron scattering literature [7,36,37]. In conjunction with Fay Tuddenham, these models have been adapted for QHAS experiments [38,39], as summarised below. 


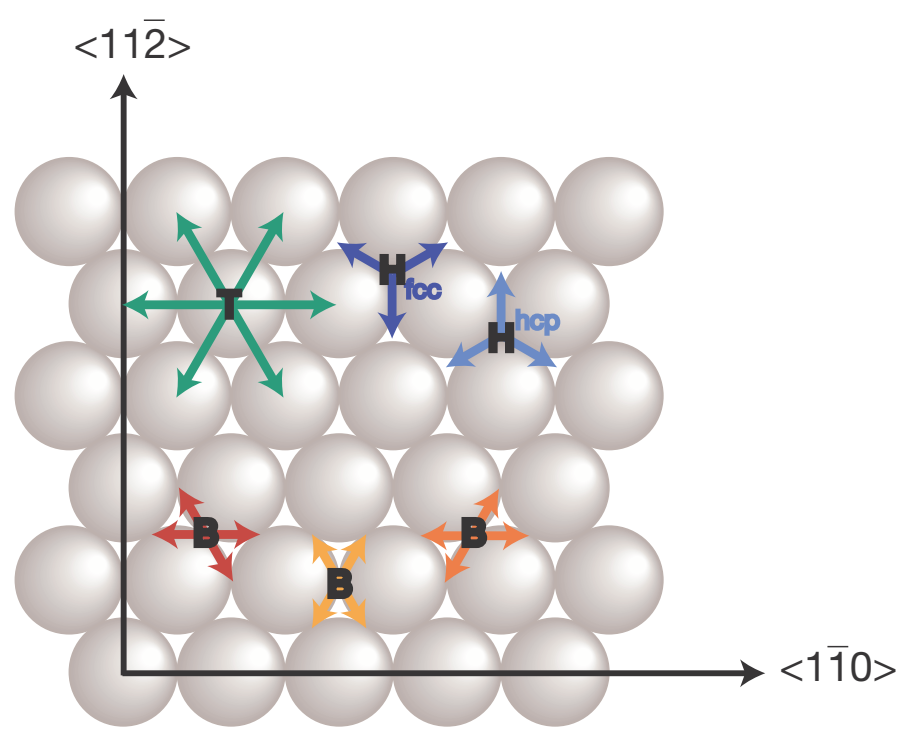

Figure 2.8: Schematic representation of the $\mathrm{Cu}(111)$ surface. The possible jump vectors for single jump diffusion between equivalent sites are shown in green for top sites, blue for hollow sites and red for bridge sites. Furthermore, the two main crystal azimuths are defined.
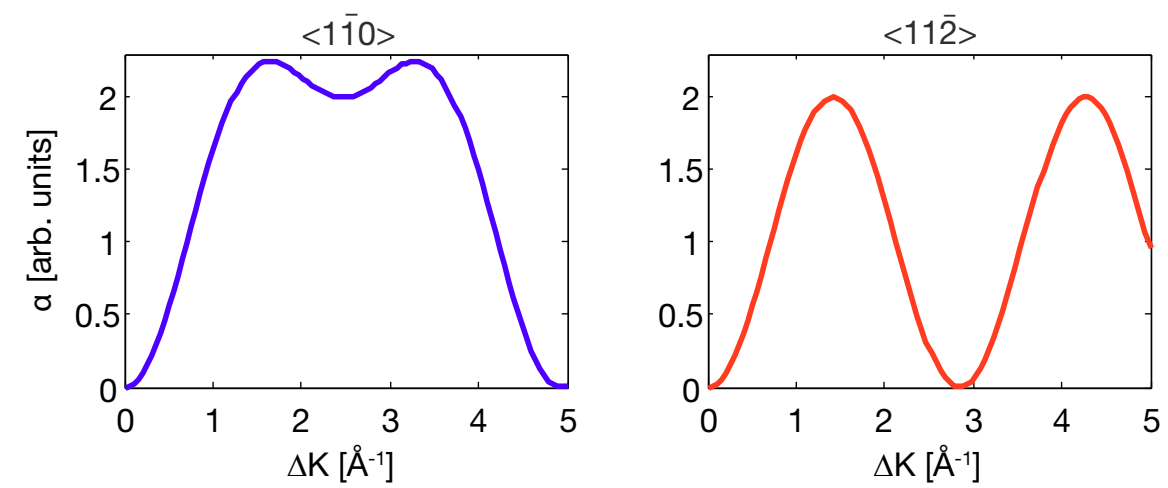

Figure 2.9: $\alpha(\Delta \boldsymbol{K})$ dependence for hopping on a Bravais lattice of top sites (or equivalently one kind of hollow sites) on $\mathrm{Cu}(111)$. The projection of the jumps along the two crystal directions, $\langle 1 \overline{1} 0>$ and $<11 \overline{2}>$, is different, as reflected in the periodicity of the blue and red curves.

In the non-Bravais jump diffusion model, the ISF is derived from a matrix, $\boldsymbol{A}$, describing all possible jump vectors. A non-Bravais lattice consists of $m$ different Bravais sub-lattices. Each jump is defined by a vector, $\boldsymbol{l}_{i j k}$, connecting the $i^{\text {th }}$ and $j^{\text {th }}$ sub-lattices and has a jump frequency, $\nu_{i j k}$, which is the inverse of the residence time of the adsorbate in each site, $\tau_{i j k}$. The total jump rate from an $i$ site to any adjacent $j$ site is given by a 
sum over all $n_{i j}$ possible jumps,

$$
\nu_{i j}=\sum_{k=1}^{n_{i j}} \nu_{i j k} .
$$

The elements of matrix $\boldsymbol{A}$, which is an $(m \times m)$ matrix, are given by

$$
A_{i j}=\sum_{k} \nu_{j i k} \exp \left(-i \Delta \boldsymbol{K} \cdot \boldsymbol{l}_{i j k}\right)-\delta_{i j} \sum_{j^{\prime}} \nu_{i j^{\prime}}
$$

with $k$ iterating over the different possible jump vectors. $\boldsymbol{A}$ can be transformed into a Hermitian matrix via

$$
B=\boldsymbol{T A} \boldsymbol{T}^{-1}
$$

where $\boldsymbol{T}$ is the similarity transformation,

$$
\boldsymbol{T}_{i j}=\sqrt{\frac{1}{c_{i}}} \delta_{i j}
$$

As described in [38], the intermediate scattering function can then be calculated as

$$
I(\Delta \boldsymbol{K}, t)=\sum_{p} w_{p}(\Delta \boldsymbol{K}) \exp (\alpha(\Delta \boldsymbol{K}) \cdot t)
$$

The prefactors, $w_{p}(\Delta \boldsymbol{K})$, are given by

$$
w_{p}(\Delta \boldsymbol{K})=\left|\sum_{i} \sqrt{c_{i}} b_{i}^{p}\right|^{2},
$$

where $c_{i}$ is the concentration of adsorbates on sites of lattice $i$ and $b_{i}^{p}$ the $p^{t h}$ eigenvector of matrix $\boldsymbol{B}$. In contrast to the simple model by Chudley \& Elliot, the ISF for hopping on a non-Bravais lattice is a sum of $m$ exponential functions, where $m$ is determined by the number of sub-lattices, with the weights $w_{p}$ describing the contribution of each exponential to the ISF. Typically, some exponential functions have zero intensity $\left(w_{p}=0\right)$, so that the number of decays is $\leq m$. In Chapters 5 and 6 , hopping on hollow and/or bridge sites on a $\mathrm{Cu}(111)$ surface is discussed. Therefore, the models for these particular non-Bravais lattices are summarised below. 


\section{Jump diffusion on threefold hollow sites}

In the case of hollow sites, hcp and fcc sites form two separate sub-lattices, thus $m$ equals

2. The analytical solution for this model, as derived in [38], is as follows:

$$
\begin{aligned}
& I(\Delta \boldsymbol{K}, t)_{<1 \overline{1} 0>}=\frac{c_{1}}{n_{1}}\left|1-\lambda \frac{4 \cos \left(\frac{\Delta \boldsymbol{K} a}{2}\right)+2}{3 \lambda-3+z}\right|^{2} \exp \left(-\frac{\nu_{12}}{6 \lambda}(3 \lambda+3+z) t\right) \\
& +\frac{c_{1}}{n_{2}}\left|1-\lambda \frac{4 \cos \left(\frac{\Delta \boldsymbol{K} a}{2}\right)+2}{3 \lambda-3-z}\right|^{2} \exp \left(-\frac{\nu_{12}}{6 \lambda}(3 \lambda+3-z) t\right) \\
& n_{1,2}=1+\lambda\left(\frac{4 \cos \left(\frac{\Delta \boldsymbol{K} a}{2}\right)+2}{3 \lambda-3 \pm z}\right)^{2} \\
& z=\sqrt{9 \lambda^{2}+16 \lambda \cos ^{2}\left(\frac{\Delta \boldsymbol{K} a}{2}\right)+16 \lambda \cos \left(\frac{\Delta \boldsymbol{K} a}{2}\right)-14 \lambda+9} \\
& I(\Delta \boldsymbol{K}, t)_{<11 \overline{2}>}=\frac{c_{1}}{m_{1}}\left|1-2 \lambda \frac{\exp \left(i \frac{\Delta \boldsymbol{K} a}{\sqrt{3}}\right)+2 \exp \left(-i \frac{\Delta \boldsymbol{K} a}{2 \sqrt{3}}\right)}{3(\lambda-1+y)}\right|^{2} \exp \left(-\frac{\nu_{12}}{2 \lambda}(\lambda+1+y) t\right) \\
& +\frac{c_{1}}{m_{2}}\left|1-2 \lambda \frac{\exp \left(i \frac{\Delta \boldsymbol{K} a}{\sqrt{3}}\right)+2 \exp \left(-i \frac{\Delta \boldsymbol{K} a}{2 \sqrt{3}}\right)}{3(\lambda-1-y)}\right|^{2} \exp \left(-\frac{\nu_{12}}{2 \lambda}(\lambda+1-y) t\right) \\
& m_{1,2}=1+4 \lambda\left|\frac{\exp \left(i \frac{\Delta \boldsymbol{K} a}{\sqrt{3}}\right)+2 \exp \left(-i \frac{\Delta \boldsymbol{K} a}{2 \sqrt{3}}\right)}{3(\lambda-1 \pm y)}\right|^{2} \\
& y=\sqrt{\lambda^{2}+\frac{2 \lambda}{9}\left(8 \cos \left(\frac{\sqrt{3} \Delta \boldsymbol{K} a}{2}\right)+1\right)+1}
\end{aligned}
$$

The precise form of these equations is of little importance at this point, but it should be noted that the ISFs for both azimuths consist of a sum of two exponential functions. The lattice constant $a$ - which is $2.55 \AA$ for $\mathrm{Cu}(111)$ [40] - defines the periodicity of the prefactors and exponentials with $\Delta \boldsymbol{K}$, while the absolute height of the curves is given by the jump frequency, $\nu_{12}$. The parameter $\lambda$, which is the ratio between the jump rates from fcc to hcp and from hcp to fcc sites, determines the relative ratios of prefactors and exponentials, and is given by the adsorption energy difference, $\Delta E$, of the sites through

$$
\lambda=\exp \left(\frac{\Delta E}{k_{B} T}\right),
$$


where $k_{B}$ is the Boltzmann constant and $T$ the temperature. $\Delta E=0$ implies $\lambda$ equal to 1 , while $\lambda>1$ for energetically different sites.

As is apparent in Figure 2.8, the jump vectors from fcc and hcp sites give the same projection along the $<1 \overline{1} 0>$ azimuth, yet different projections in the $<11 \overline{2}>$ direction. The ISF therefore exhibits a single exponential function in the $<1 \overline{1} 0>$ azimuth when both hollow sites are equivalent, as illustrated in the top panels of Figure 2.10 where the intensity of the fast decay is zero for all momentum transfer values. For energetically different sites, the second decay gains in intensity, as apparent in the lower panels where results for increasing $\lambda$ are shown. Along the $<11 \overline{2}>$ azimuth, a sum of two exponentials is observed at certain $\Delta \boldsymbol{K}$ positions for all values of $\lambda$, as shown in Figure 2.11.

\section{Jump diffusion on twofold bridge sites}

Jump diffusion on a lattice of twofold bridge sites on a $\mathrm{Cu}(111)$ substrate is conceptually simpler since all bridge sites are always energetically equal. The analytical solution therefore does not include a parameter $\lambda$ and the relative ratios of the exponentials and prefactors are constant:

$$
\begin{aligned}
I(\Delta \boldsymbol{K}, t)_{<1 \overline{1} 0>} & =c_{1} \frac{\left(2 \cos \left(\frac{\Delta \boldsymbol{K} a}{4}\right)-2\right)^{2}}{4 \cos ^{2}\left(\frac{\Delta \boldsymbol{K} a}{4}\right)+2} \exp \left(-3 \nu_{12} t\right) \\
& +c_{1} \frac{\left(2 \cos \left(\frac{\Delta \boldsymbol{K} a}{4}\right)+1\right)^{2}}{2 \cos ^{2}\left(\frac{\Delta \boldsymbol{K} a}{4}\right)+1} \exp \left(-\nu_{12}\left(1-\cos \left(\frac{\Delta \boldsymbol{K} a}{2}\right)\right) t\right) \\
I(\Delta \boldsymbol{K}, t)_{<11 \overline{2}>} & =\frac{c_{1}}{y^{2}+y}\left(2 \cos \left(\frac{\Delta \boldsymbol{K} a \sqrt{3}}{4}\right)+1+y\right)^{2} \exp \left(-\frac{(3-y) \nu_{12}}{2} t\right) \\
& +\frac{c_{1}}{y^{2}-y}\left(2 \cos \left(\frac{\Delta \boldsymbol{K} a \sqrt{3}}{4}\right)+1-y\right)^{2} \exp \left(-\frac{(3+y) \nu_{12}}{2} t\right) \\
y & =\sqrt{8 \cos ^{2}\left(\frac{\Delta \boldsymbol{K} a \sqrt{3}}{4}\right)+1}
\end{aligned}
$$

While there are three symmetrically different bridge sites (cf. Figure 2.8), i.e. $m=3$, they only give two different projections in both crystal directions and one of the exponentials along both azimuths has zero intensity. The resulting exponentials and normalised prefactors are presented in Figure 2.12. 

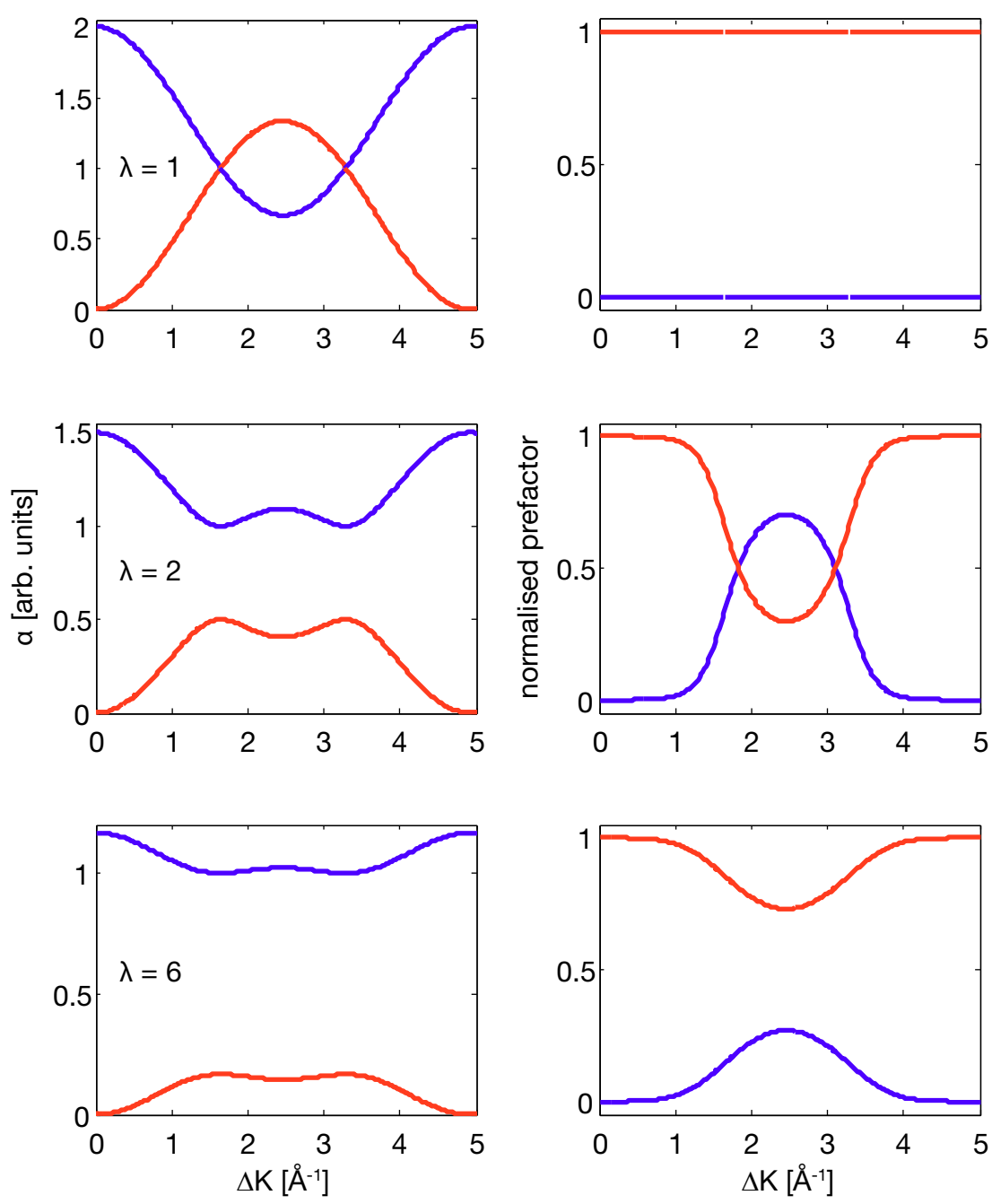

Figure 2.10: Illustration of the jump diffusion model for hopping on fcc and hep hollow sites on $\mathrm{Cu}(111)$, projected onto the $<1 \overline{1} 0>$ azimuth. The $\Delta \boldsymbol{K}$ dependence of the two exponentials (left column) and corresponding normalised prefactors in matching colours (right column) are presented for degenerate sites $(\lambda=1)$ in the top panels, and for increasingly non-degenerate sites beneath $(\lambda=2$ and $\lambda=6)$.

\section{Jump diffusion on hollow and bridge sites}

In Chapter 6, a third case of hopping on a non-Bravais lattice is discussed, namely hopping from bridge to hollow site and vice versa. A total of five different sites and three different energy levels thus needs to be considered, expressed as a $(5 \times 5) \boldsymbol{B}$ matrix. Due to the complexity of the problem, the ISF was not calculated analytically but instead a numerical solution was determined by defining $\boldsymbol{A}$, performing the similarity transform to obtain $\boldsymbol{B}$ and subsequently calculating the eigenvalues and eigenvectors of $\boldsymbol{B}$. 

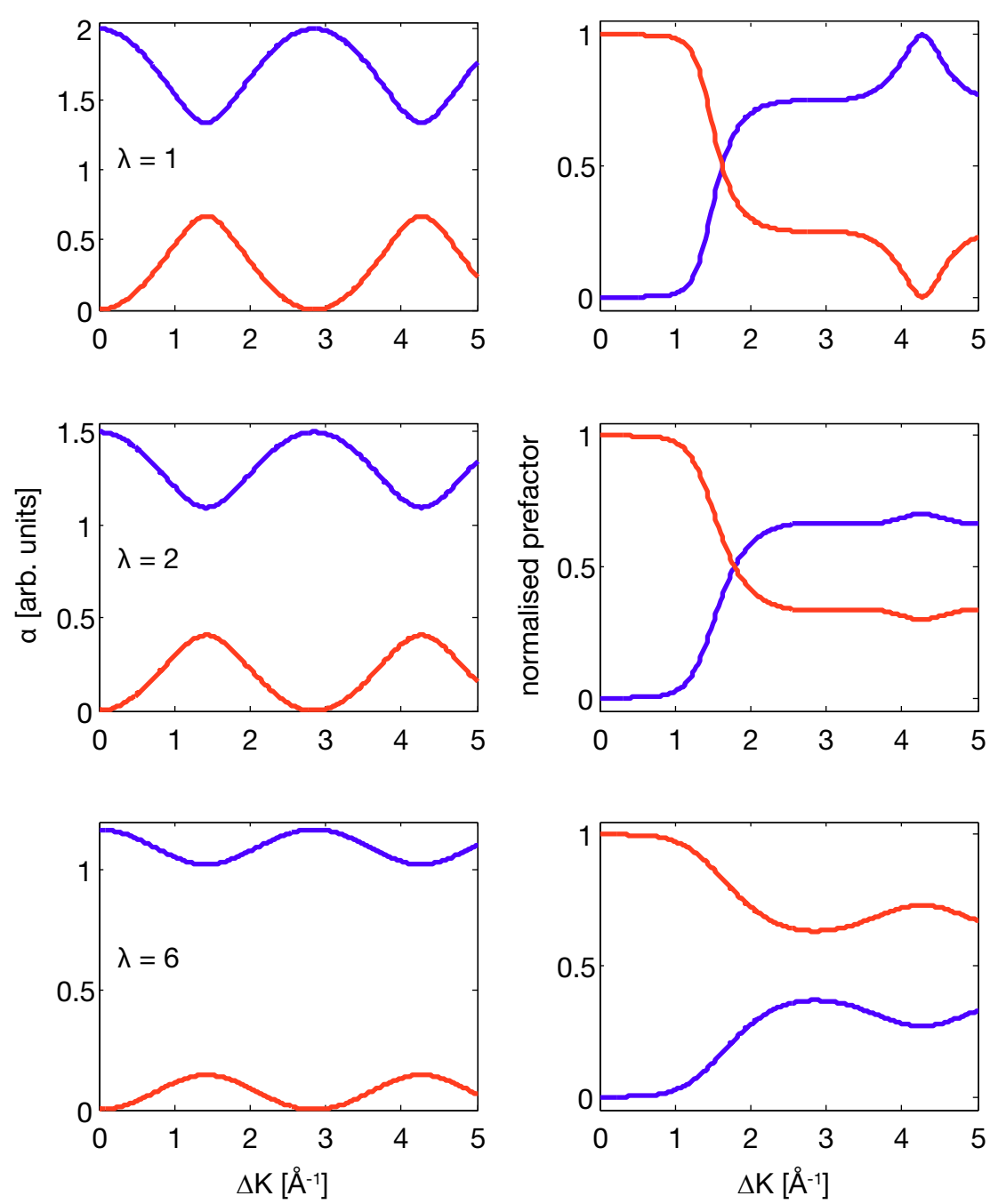

Figure 2.11: Illustration of the jump diffusion model for hopping on fcc and hcp hollow sites on $\mathrm{Cu}(111)$, projected onto the $<11 \overline{2}>$ azimuth. The $\Delta \boldsymbol{K}$ dependence of the two exponentials (left column) and corresponding normalised prefactors in matching colours (right column) are presented for degenerate sites $(\lambda=1)$ in the top panels, and for increasingly non-degenerate sites beneath $(\lambda=2$ and $\lambda=6)$.

Two $\lambda$ parameters define the level of degeneracy between the different sites, $\lambda_{1}$ for the relative energy of bridge sites and one type of hollow sites, and $\lambda_{2}$ for bridge sites and the other type of hollow sites. In the case of degeneracy between fcc and hcp sites $\left(\lambda_{1}=\lambda_{2}\right)$, all intensity goes into three exponentials along $<1 \overline{1} 0>$ and four along $<11 \overline{2}>$, as shown in Figures 2.13 and 2.14, respectively. Keeping the hollow sites degenerate but varying the energy difference between hollow and bridge sites, the ratio of the exponentials (left column in the Figures) changes, opening a gap when approaching either extreme of adsorption on hollow sites $(\lambda \rightarrow \infty)$ or bridge sites alone $(\lambda \rightarrow 0)$. When $\lambda_{1}=\lambda_{2}=1.5$, 

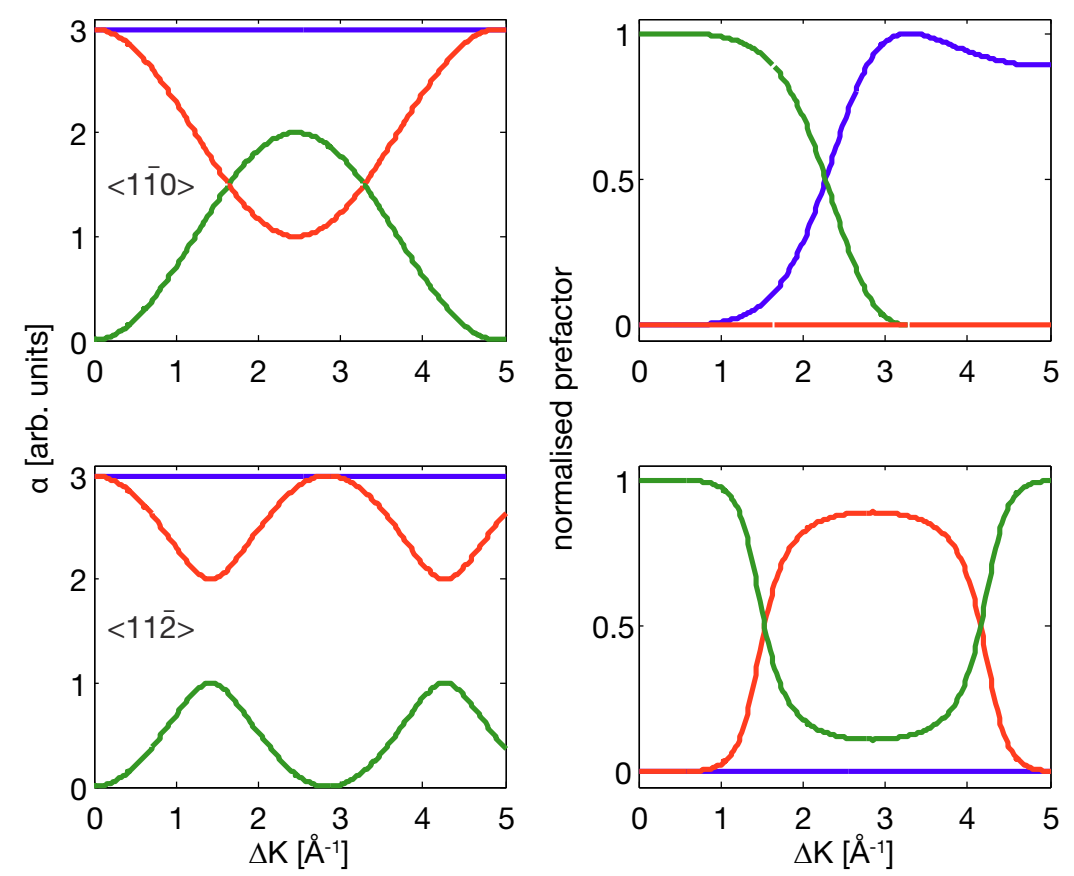

Figure 2.12: Illustration of the jump diffusion model for hopping on bridge sites on $\mathrm{Cu}(111)$, projected onto the $<1 \overline{1} 0>$ (top row) and $<11 \overline{2}>$ azimuths (bottom row). The $\Delta \boldsymbol{K}$ dependence of the three exponentials (left column) and corresponding normalised prefactors in matching colours (right column) are presented.

bridge and hollow sites are weighted equally, as there are three bridge but only two hollow sites in a unit cell, and maximum symmetry of the exponentials is reached (cf. centre panels in Figures 2.13 and 2.14).

When neither $\lambda$ parameters equal 1 , all five components contribute to the total intensity, yielding a sum of five exponentials in the ISF. Varying either one or both $\lambda$ parameters, changes the ratio of the different exponentials, generating large gaps when a high level of degeneracy is obtained (cf. Figures 2.15 and 2.16).

Finally, it should be noted that a single exponential decay is observed at approximately $\Delta \boldsymbol{K}<0.7 \AA$ for all of these jump diffusion models. Experiments where a simple lineshape is desired to facilitate interpretation of the data, such as, for example, in the investigation of the temperature dependence of the dephasing rate at fixed momentum transfer, should therefore be performed in this regime (cf. Section 5.4.3). 

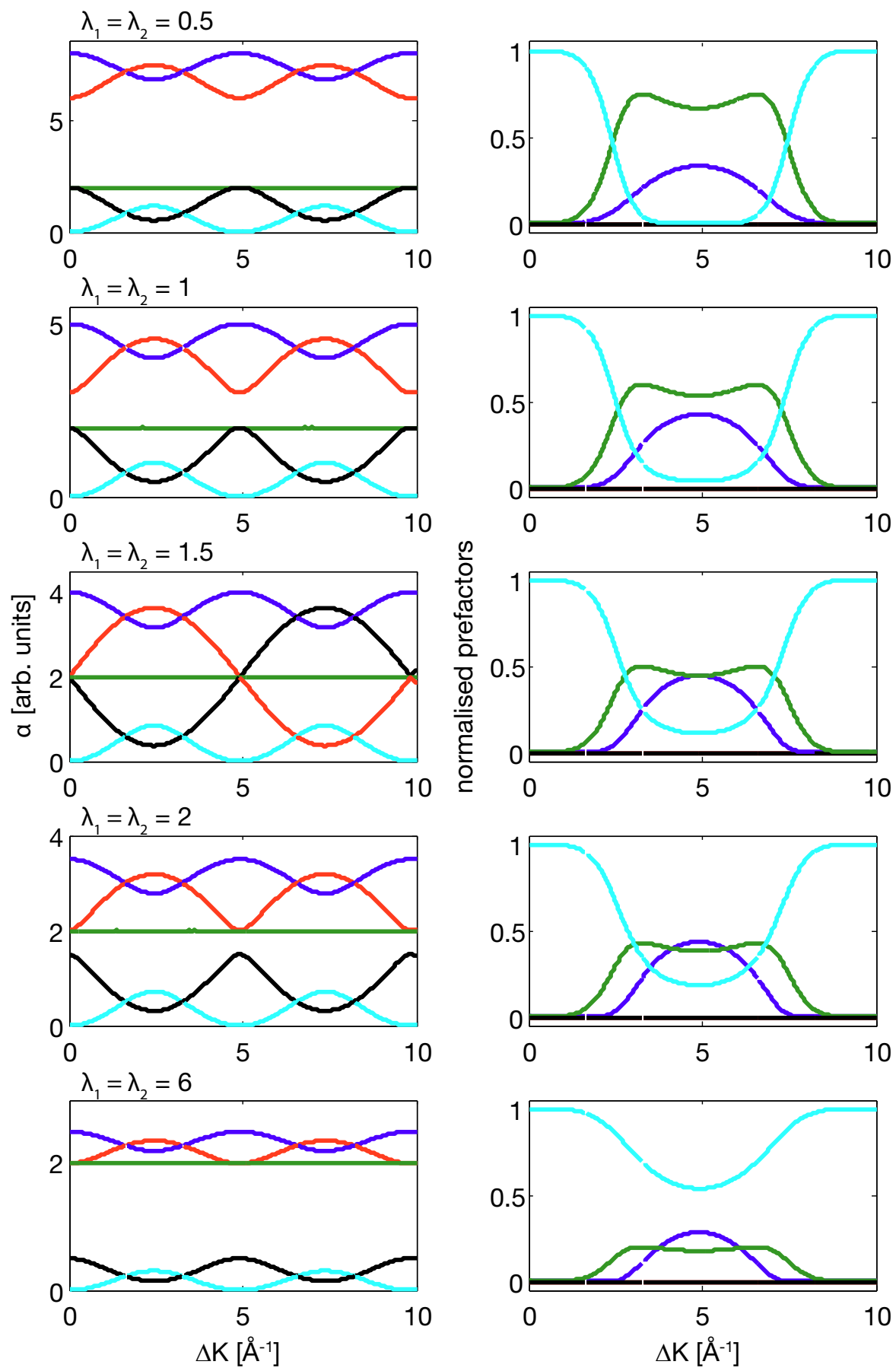

Figure 2.13: Illustration of the jump diffusion model for hopping on bridge and degenerate fcc and hcp hollow sites on $\mathrm{Cu}(111)$, projected onto the $<1 \overline{1} 0>$ azimuth. The $\Delta \boldsymbol{K}$ dependence of the exponentials (left column) and corresponding normalised prefactors in matching colours (right column) are presented for varying levels of degeneracy between bridge and hollow sites. $\lambda_{1}=\lambda_{2}=1$ is the case of degenerate bridge, fcc and hcp sites. 

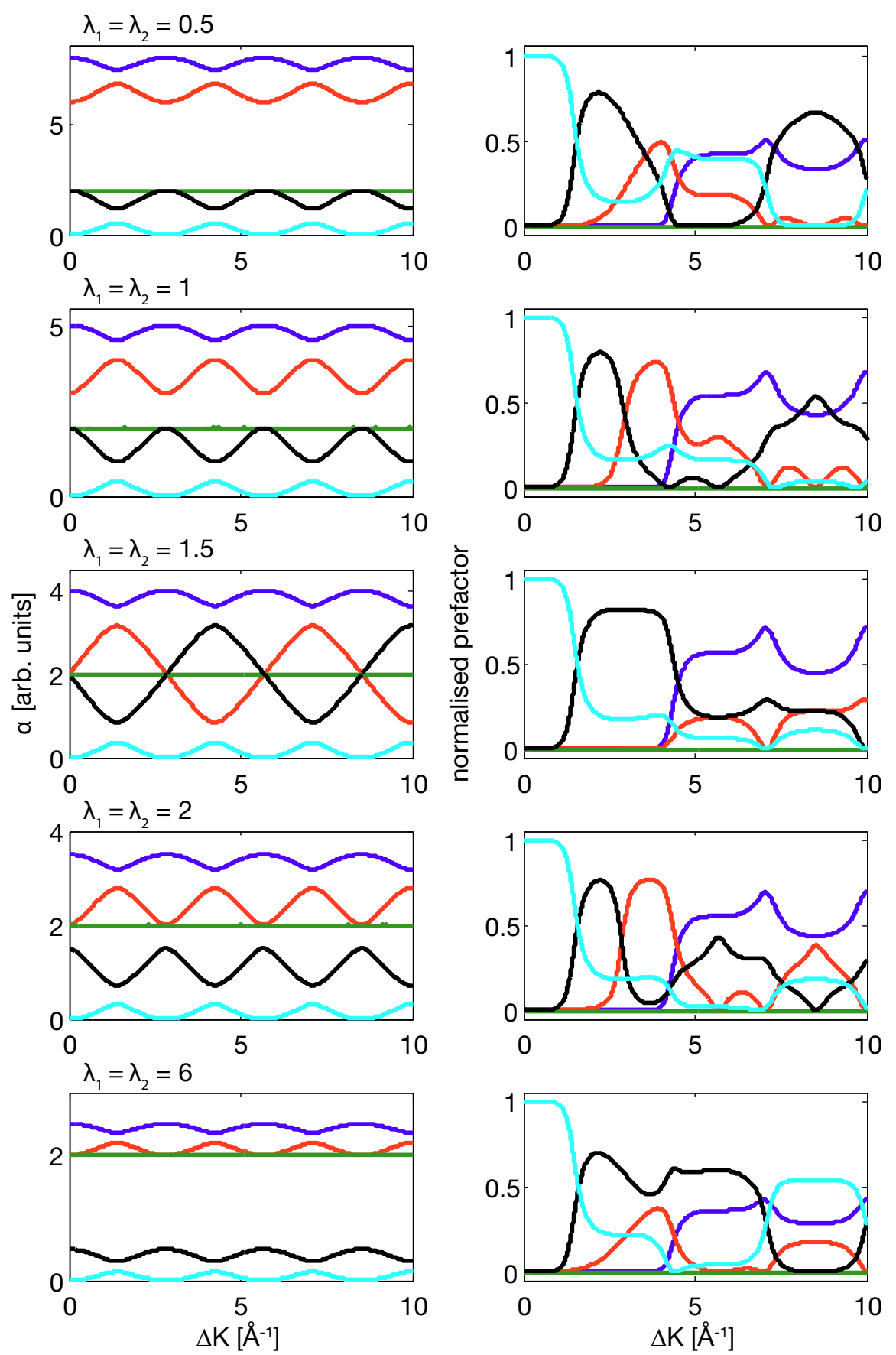

Figure 2.14: Illustration of the jump diffusion model for hopping on bridge and degenerate fcc and hcp hollow sites on $\mathrm{Cu}(111)$, projected onto the $<11 \overline{2}>$ azimuth. The $\Delta \boldsymbol{K}$ dependence of the exponentials (left column) and corresponding normalised prefactors in matching colours (right column) are presented for varying levels of degeneracy between bridge and hollow sites. $\lambda_{1}=\lambda_{2}=1$ is the case of degenerate bridge, fcc and hcp sites. 

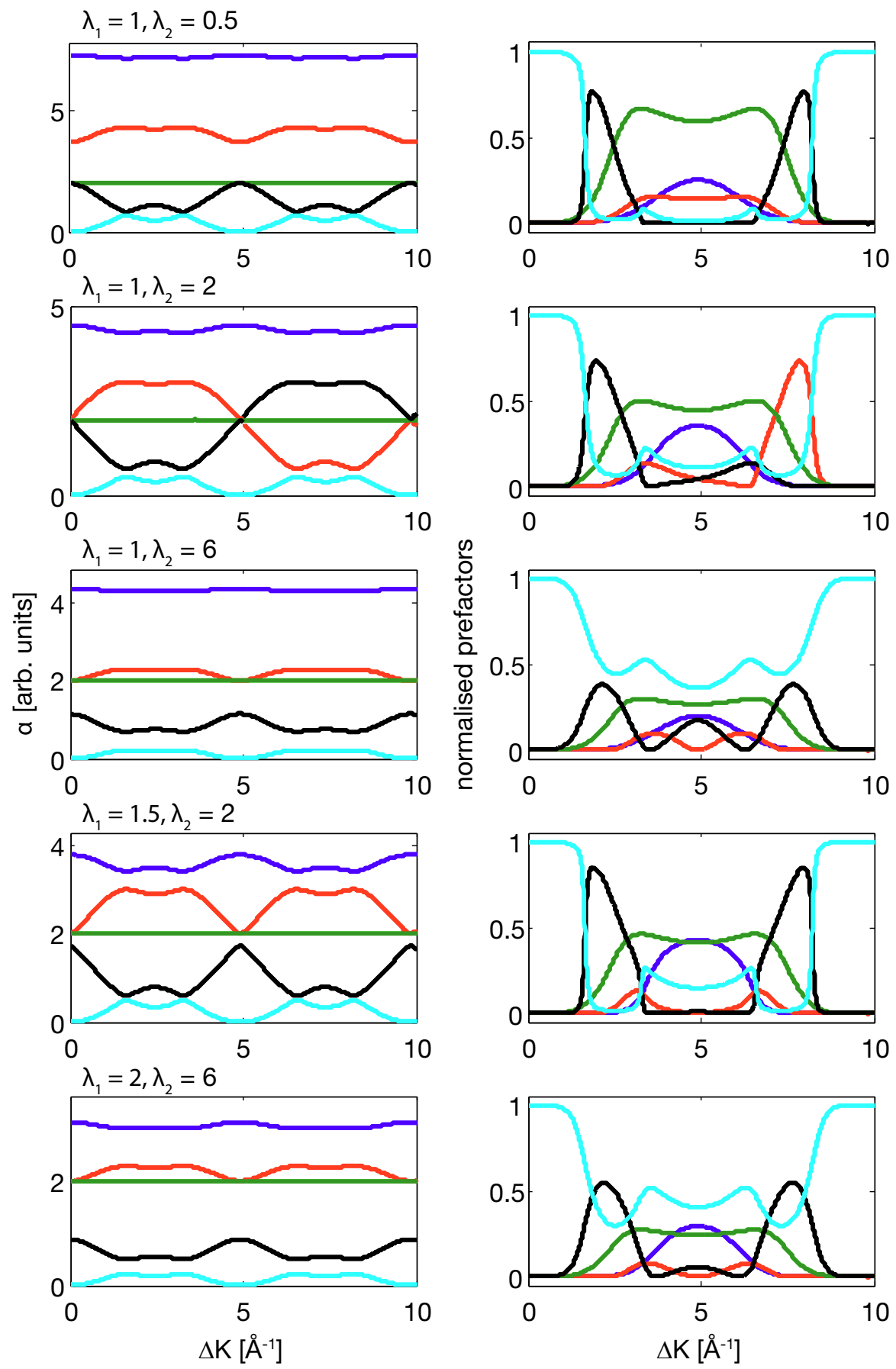

Figure 2.15: Illustration of the jump diffusion model for hopping on bridge, fcc and hcp sites on $\mathrm{Cu}(111)$, projected onto the $<1 \overline{1} 0>$ azimuth. The $\Delta \boldsymbol{K}$ dependence of the exponentials (left column) and corresponding normalised prefactors in matching colours (right column) are presented for varying levels of degeneracy between bridge, fcc and hcp sites, where $\lambda_{1}$ defines the level of degeneracy between bridge and one type of hollow sites, while $\lambda_{2}$ relates bridge and the other type of hollow site. 

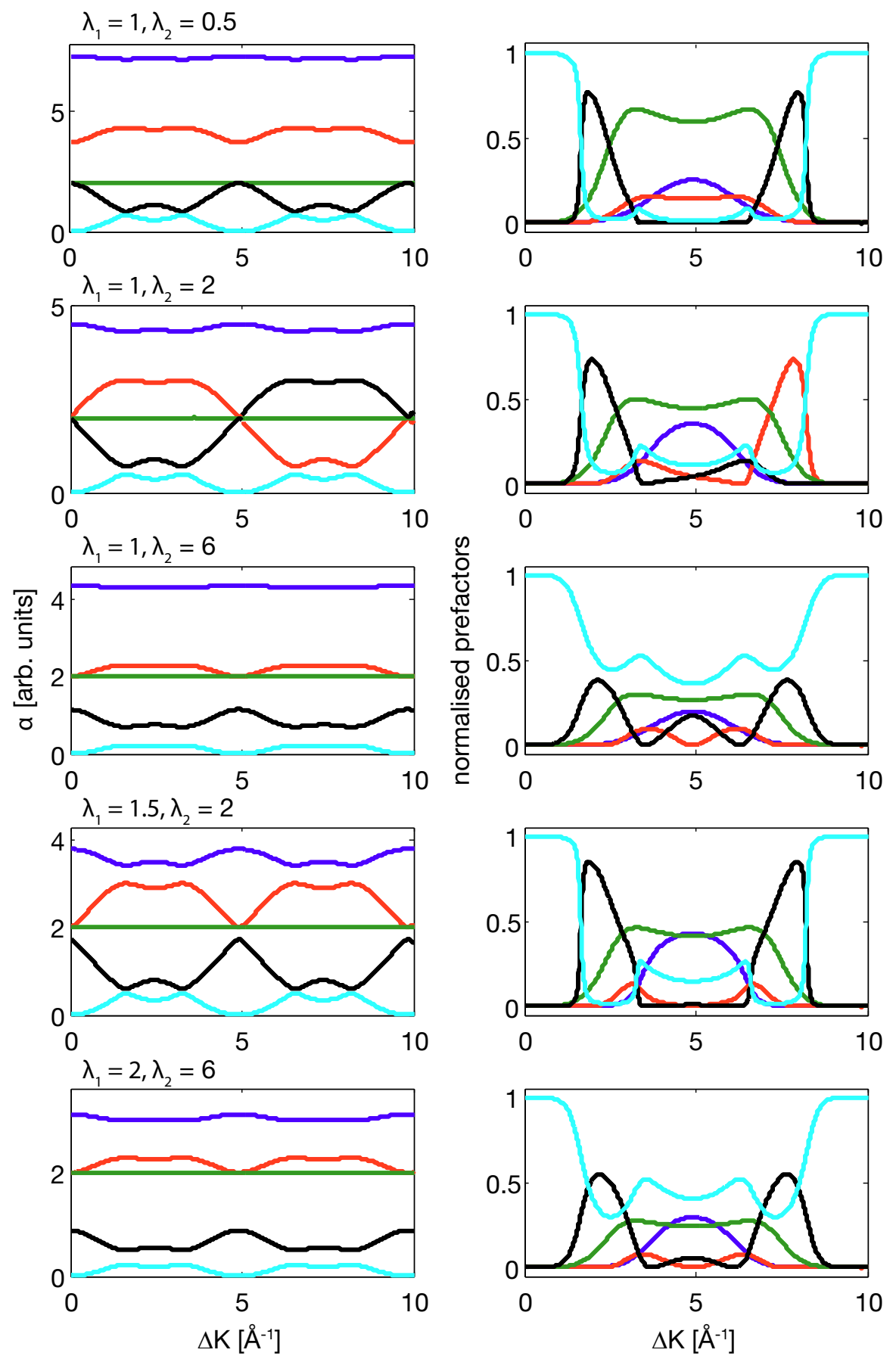

Figure 2.16: Illustration of the jump diffusion model for hopping on bridge, fcc and hcp sites on $\mathrm{Cu}(111)$, projected onto the $<11 \overline{2}>$ azimuth. The $\Delta \boldsymbol{K}$ dependence of the exponentials (left column) and corresponding normalised prefactors in matching colours (right column) are presented for varying levels of degeneracy between bridge, fcc and hcp sites, where $\lambda_{1}$ defines the level of degeneracy between bridge and one type of hollow sites, while $\lambda_{2}$ relates bridge and the other type of hollow site. 


\subsubsection{Lateral interactions: De Gennes narrowing}

Repulsive lateral interactions amongst adsorbates lead to a deviation from the $\alpha(\Delta \boldsymbol{K})$ curves predicted by analytical models described in the previous section, as first reported by de Gennes in 1959 [41]. Adsorbates repelling each other prefer a long-range quasihexagonal structure. The result is a preferred average distance between the adsorbates - dependent on the coverage - and reduced mobility on these length scales. When adsorbates approach each other, on the other hand, their mobility increases compared to that of non-repelling species. This effect creates a dip feature, termed "de Gennes narrowing", and a peak at lower $\Delta \boldsymbol{K}$ overlayed over the standard $\alpha(\Delta \boldsymbol{K})$ curves, as illustrated in Figure 2.17. The grey line shows the result from a molecular dynamics (MD) simulation for a single adsorbate particle, hence it includes no repulsive interactions and a smooth sinusoidal curve is observed. The red line presents an equivalent simulation for 40 interacting adsorbates. A peak appears at low $\Delta \boldsymbol{K}$ values, illustrating increased mobility of certain length scales, while a dip occurs at the length scale of the quasi-hexagonal arrangement.

The position of the dip can be used to estimate the coverage,

$$
\Theta=\frac{a^{2}}{r^{2}}
$$

from the average nearest-neighbour distance, $r$, and the substrate lattice constant, $a$ [31]. By approximating the local order as a hexagonal lattice [31], the nearest-neighbour distance can be deduced from the momentum transfer position of the de Gennes dip, $\Delta K_{\text {dip }}$, as

$$
r=\frac{4 \pi}{\sqrt{3} \cdot \Delta \boldsymbol{K}_{d i p}} .
$$

\subsubsection{Molecular dynamics simulations}

In addition to interpreting experimental data using analytical models for diffusion, MD simulations can be performed which give a more complete picture of the motion, providing information about the nature of lateral interactions and the friction as well as the type of diffusion [42-44]. In a classical simulation, the diffusion of an adsorbate particle is 


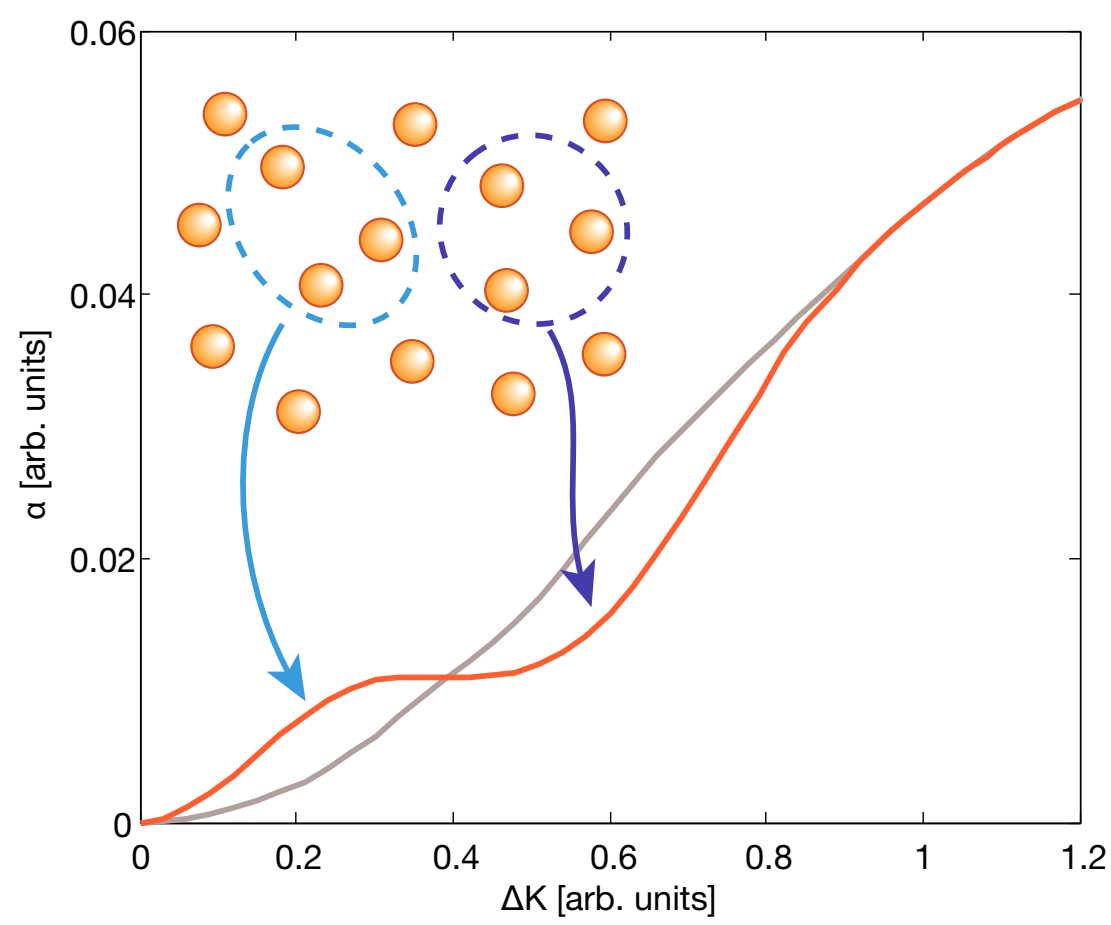

Figure 2.17: Schematic illustration of the effect lateral interactions have on the $\alpha(\Delta \boldsymbol{K})$ curve in a HeSE experiment. A single particle MD simulation, presented in grey, produces a sinusoidal curve, while an identical simulation of 40 interacting adsorbates, shown in red, exhibits a de Gennes peak and dip feature. The peak can be explained by increased mobility of adsorbates at certain length scales due to strong repulsion, as marked in cyan, while the dip (de Gennes narrowing) is evidence for decreased mobility where a quasi-hexagonal arrangement is found, shown in blue.

described using the 2D Langevin equation

$$
m \ddot{\boldsymbol{R}}_{i}=-\nabla V\left(\boldsymbol{R}_{i}\right)-m \eta \dot{\boldsymbol{R}}_{i}+\xi(t)+\sum_{i \neq j} F_{i, j}
$$

where the trajectories for the $i$-th adsorbate particle with velocity $\dot{\boldsymbol{R}}_{i}$ are calculated for motion on a potential energy surface (PES), $V\left(\boldsymbol{R}_{i}\right)$. The friction coefficient, $\eta$, describes the energy exchange between adsorbate and substrate and a random impulse term, $\xi$, ensures that the total energy of the system remains preserved. For simulations of multiple adsorbate particles, $F_{i, j}$ models the pairwise lateral interactions between particle $i$ and particle $j$, for example by dipole-dipole interactions. The input parameters $V\left(\boldsymbol{R}_{i}\right), \eta$ and $F_{i, j}$ are varied iteratively to obtain a realistic description of the experimental data. 
In addition to interpreting experimental data, MD simulations can be used to study the effect of individual parameters on the adsorbate system, which is often not possible in an experiment. Varying the friction coefficient while keeping all other parameters constant, for example, provides information about the rate of energy exchange between adsorbate and substrate. Transition state theory (TST) is widely used to describe diffusion through the population of the transition state, determining the rate of diffusion. TST assumes that every atom in a transition state originates from an adjacent adsorption well, which is only true in certain cases. In most real systems, a deviation from TST is observed, as first discussed by Kramers in 1940 [45]. The jump frequency, $\nu=1 / \tau$, as a function of $\eta$ is shown in Figure 2.18 [46]. A corresponding curve obtained by MD simulations for $\mathrm{Cp} / \mathrm{Cu}(111)$ is presented in Chapter 5. In the case of low friction, the rate of energy exchange between adsorbate and substrate is small and once the diffusing species has acquired enough energy to overcome the barrier, it "rollercoasters" over many barriers. This implies only a fraction of the species in the transition state comes from the adjacent well and thus the actual jump frequency is lower than predicted by TST. In the case of high friction, the diffusing particle changes its energy, and hence its velocity, so frequently that it does not move by a direct route between adsorption sites but rather moves by a random walk leading to re-crossings of the transition state. Since the same atom can be in the transition state a number of times, assuming that each atom in the transition state has just come from the adjacent adsorption site yet again overestimates the jump rate. TST therefore only gives a realistic description for systems at the "turnover" point between low and high friction, i.e. on the peak of the curve.

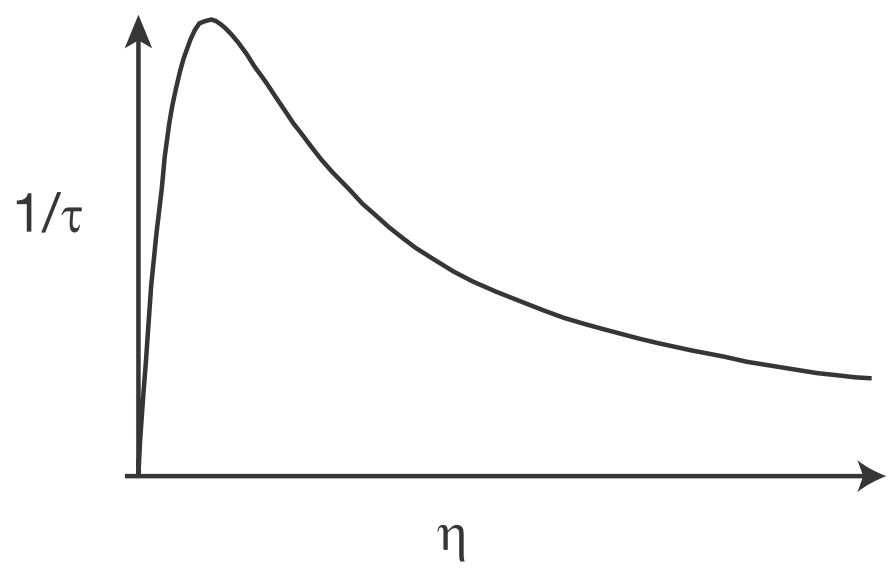

Figure 2.18: Jump frequency as a function of friction, illustrating the "turnover" from low to high friction (from [46]). 


\section{Chapter 3}

\section{A new helium atom scattering apparatus}

A new compact high-resolution helium atom scattering (HAS) spectrometer has been developed to enable sample preparation and increase the throughput of the HeSE spectrometer. The instrument has been termed "MiniScat", short for miniature atom scattering apparatus. This chapter gives the motivation for such a new piece of equipment, summarises the main design aspects and presents the experimental characterisation of the spectrometer. The overall geometry of the instrument and the mechanical details of frame and vacuum chambers were designed by Andrew Jardine.

\subsection{Motivation}

Due to the uniqueness of the Cambridge helium-3 spin-echo (HeSE) spectrometer its uptime is high in demand, creating the need for selective and efficient scheduling of experiments. In general, preparation work and structural studies are kept to a minimum on the HeSE instrument in order to maximise the time it can be used for surface dynamics measurements.

In the case of widely studied crystal surfaces and simple adsorbate species, information from the literature can be used to plan HeSE experiments. The aim of the present thesis, however, is to extend the applications of HeSE towards more complex adsorbate systems, investigating the motion of larger adsorbates such as organic molecules. Furthermore, work has recently been extended to studies of non-metal substrate surfaces, for example $\mathrm{TiO}_{2}$ [27]. Such an expansion in the variety of adsorbate systems invariably leads to some 
that little is known about. The need for more detailed preliminary work to establish a reproducible surface preparation method and initial measurements to study the adsorption behaviour of these systems thus arises, often demanding more measurement time than the actual dynamics experiments. Moreover, comparative studies of similar adsorbate systems become necessary for the broader understanding of surface processes in complex systems, such as the five-membered aromatic molecules presented in Chapters 5, 6 and 7, again increasing the demand for a larger experimental throughput on the HeSE spectrometer. Therefore, a separate helium atom scattering spectrometer, MiniScat, has been designed and constructed to reduce the demand for instrument time on the HeSE experiment and to increase the experimental throughput.

Diffraction studies give a picture of static structures on surfaces thus probing longrange ordering effects, whereas HeSE, on the other hand, measures the diffusion which mainly probes the short-range order. Combining these two techniques provides a much more complete picture of the interaction potential of adsorbate systems. On MiniScat, diffraction and uptake measurements can be performed using a helium- 4 beam. While the original HeSE detector is a factor of 50 more sensitive than the commercial mass spectrometer used on MiniScat [23] and the new HeSE detector increases the sensitivity by another three orders of magnitude [30], the smaller instrument has the advantage of a wider angular spread in the beam. With an angular spread of $0.1^{\circ}$ [23], specular and diffraction peaks on the HeSE spectrometer are indeed so sharp that they can be missed easily due to small changes in temperature or slight mechanical misalignments. At $0.2^{\circ}$, MiniScat's angular spread is somewhat larger and thus facilitates diffraction studies compared to the HeSE spectrometer while still being sufficient to separate most diffraction peaks.

In addition to its role as a sample preparation facility, MiniScat can be used to perform experiments which cannot be carried out on the HeSE spectrometer. In contrast to the setup of the HeSE machine, a mass spectrometer is installed on the scattering chamber in between the incident and outgoing helium beam of MiniScat. Therefore, thermal desorption spectroscopy (TDS) can be carried out, recording the specular helium intensity while simultaneously studying the desorbed species in the mass spectrometer. Furthermore, the mass spectrometer can be inserted into the chamber on a z-shift, positioning it close to the sample surface to permit quantitative desorption peak analysis, which is not possible on the HeSE apparatus. MiniScat's scattering chamber also includes ports for the addition of further surface characterisation facilities, such as a low energy electron diffraction (LEED) and/or Auger spectroscopy system. 
A further application of MiniScat is the testing of new equipment for the Cambridge HeSE spectrometer. MiniScat is designed to have its main components compatible and interchangeable with those of the HeSE spectrometer. The source and first two differential pumping stage chambers are identical on both machines, allowing facile swapping of the beam sources. The new source presented in Chapter 4 has initially been tested and characterised on MiniScat before it was installed on the HeSE spectrometer. Similarly, the dimensions of the top port of the scattering chamber and the height above the beam are identical on both instruments, which allowed the development of a new manipulator and sample transfer system [27]. Such equipment development and testing would be difficult on the HeSE spectrometer, putting the instrument out of action for weeks or months at a time.

Finally, MiniScat could be used for scattering of a probe other than helium-4, simply by purging the gas lines and exchanging the gas cylinder (cf. bottom left corner in Figure 3.3). Scattering heavy atoms - for example neon or argon - typically shows a more accurate level of surface corrugation than HAS, and is sometimes more sensitive to structural details [15], thus providing additional information about the surface structure.

In summary, MiniScat has been developed as a small, robust instrument to complement the Cambridge HeSE spectrometer and to facilitate the investigation of more complex adsorbate dynamical systems.

\subsection{Design and experimental setup}

The overall design of MiniScat is that of a standard helium atom scattering spectrometer. A supersonic atomic beam source creates a variable energy helium beam which is scattered off the sample surface located in an ultrahigh vacuum (UHV) chamber. A detector records the scattered helium intensity as a function of scattering angle, time and temperature for diffraction, uptake and thermal desorption studies, respectively. A computer controls and records experiments and monitors the system status. A schematic drawing of the apparatus is shown in Figure 3.1.

As is implied in its name, MiniScat is an exceptionally small helium atom scattering apparatus. It has been designed in the most compact form practicable with both beam arms - from beam source to sample and from sample to detector - only $0.7 \mathrm{~m}$ in length each. Such a short overall path minimises intensity loss of the helium beam and reduces 


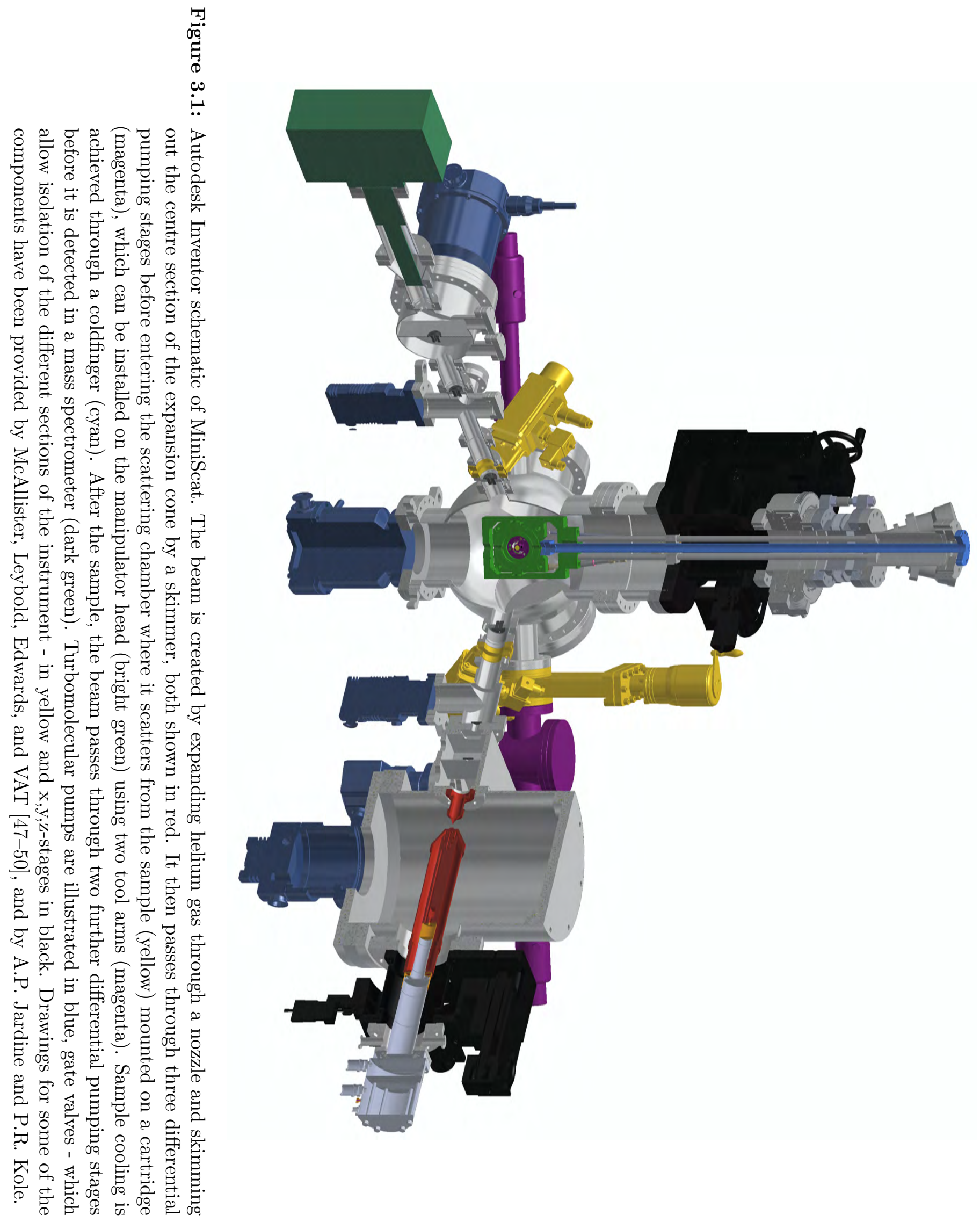


the vacuum chamber volume. Two annotated photographs of MiniScat are shown in Figure 3.2.

In Chapter 2, two types of atom scattering systems have been described, the "fixed angle" type where the angle between incident and outgoing beam is constant, and movable systems which have a rotating detector arm to alter the total scattering angle [15]. MiniScat is a fixed angle spectrometer, with a total scattering angle of $90^{\circ}$. A fixed angle instrument is much simpler and thus less prone to equipment failure and allows for more efficient differential pumping of the detector. The somewhat arbitrary choice of spectrometer angle was influenced by experimental as well as technical considerations. In general, the instrument angle should be minimised in order to maximise the accessible range of scattering angles during an experiment. On the other hand, a smaller angle between the source and detector beam arms makes construction and servicing of the instrument components increasingly difficult, particularly in combination with a short distance between source and detector.

\subsubsection{Vacuum system}

MiniScat's vacuum system is designed to achieve a difference of several orders of magnitude in helium partial pressure between beam source and detector. The high intensity helium beam is created by supersonic expansion of pressurised helium gas through a nozzle into a high vacuum chamber and subsequent skimming out of the centre portion (cf. Chapter 4). The remaining helium gas needs to be pumped away, maintaining a low pressure in the source chamber to avoid attenuation of the beam. Therefore, two turbomolecular pumps with a combined pumping speed of approximately 1200 litres per second are installed on the source chamber, backed by a $22 \mathrm{l} / \mathrm{s}$ two-stage rotary vane pump. The frame of the apparatus is bolted onto the floor to ensure it could withstand the torque of $4000 \mathrm{Nm}$ in the case of rapid shutdown of the largest pump.

During an experiment, the pressure in the source chamber is typically on the order of $10^{-4}$ mbar. The scattering chamber, however, is at UHV pressure, i.e. in the $10^{-10} \mathrm{mbar}$ to $10^{-11}$ mbar range. Furthermore, the helium background pressure in the detector needs to be kept to a minimum to ensure optimum sensitivity to the scattered signal. To achieve this reduction in pressure, several differential pumping stages are included between source and scattering chamber and prior to the detector. 

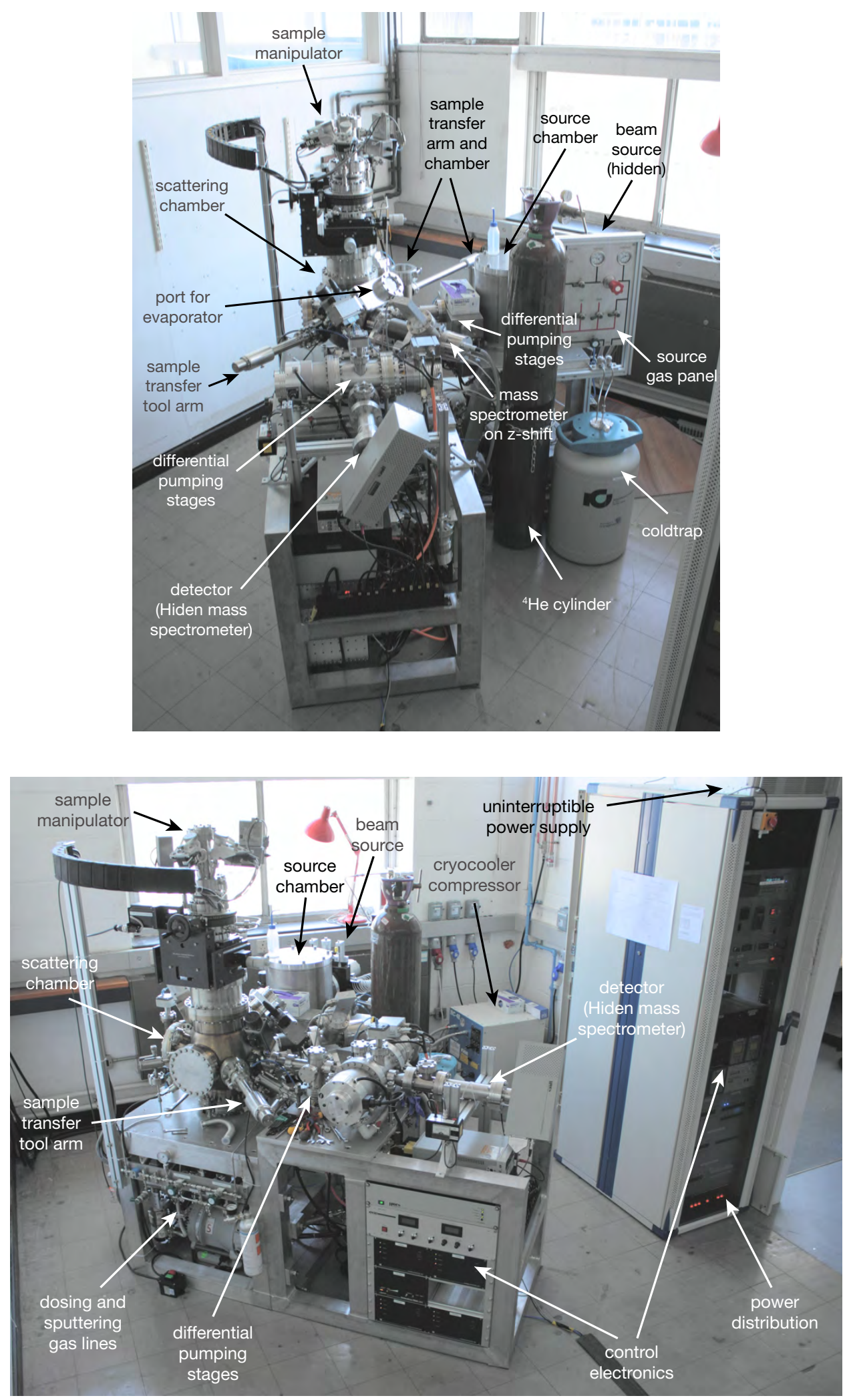

Figure 3.2: Photographs of MiniScat, annotated to illustrate its main components. The setup shown is the original setup described in the text. 
Figure 3.3 shows a schematic illustration of the vacuum system, presenting the source arm in green, the scattering chamber in blue, the gas dosing lines and sample transfer system in cyan and the detector arm in red. The diagram includes information about the vacuum pumps used, such as the make and pumping speed. On the scattering chamber and detector arm, turbomolecular pumps are connected in series to improve the total compression ratio, and oil-free pumps are used to avoid contamination of sample and UHV system. In addition, a gas flow schematic of the high pressure components for the helium beam source is included in violet in the Figure.

\section{Apertures and alignment}

Between the vacuum chambers, apertures of increasing diameter are installed along the beamline to reduce the diffuse helium background while permitting the beam to pass through. A $0.5 \mathrm{~mm}$ diameter skimmer is placed at the exit port of the source chamber. The remaining aperture sizes and lengths are summarised in Table 3.1. The apertures are dimensioned to give a helium background pressure attenuation factor of $5 \cdot 10^{7}$ between source and detector. A typical operating pressure of up to $5 \cdot 10^{-4} \mathrm{mbar}$ in the source chamber pressure, for example, thus gives a background of at most $1 \cdot 10^{-11}$ mbar helium in the detector. The spot size of the helium beam defined by these apertures is $2.9 \mathrm{~mm}$ on the sample.

\begin{tabular}{lcc}
\hline Aperture location & Aperture diameter $[\mathbf{m m}]$ & Aperture length $[\mathbf{m m}]$ \\
\hline Source pumping stages 1-2 & 1.0 & 2.0 \\
Source pumping stages 2-3 & 1.4 & 10.0 \\
Scattering chamber entrance & 2.3 & 20.0 \\
Scattering chamber exit & 4.0 & 20.0 \\
Detector pumping stages 1-2 & 5.2 & 20.0 \\
Detector chamber entrance & 5.9 & 20.0 \\
\hline
\end{tabular}

Table 3.1: MiniScat aperture sizes and lengths.

Alignment of the apertures has been achieved by shining a laser down the reverse beam path, diffracting at the skimmer edge. Each aperture was then added along the path defined by the laser light one at a time, while ensuring that the diffraction pattern remained symmetric. After installation of all apertures, source and detector were replaced by viewports, the system evacuated, and the alignment under vacuum tested by shining 


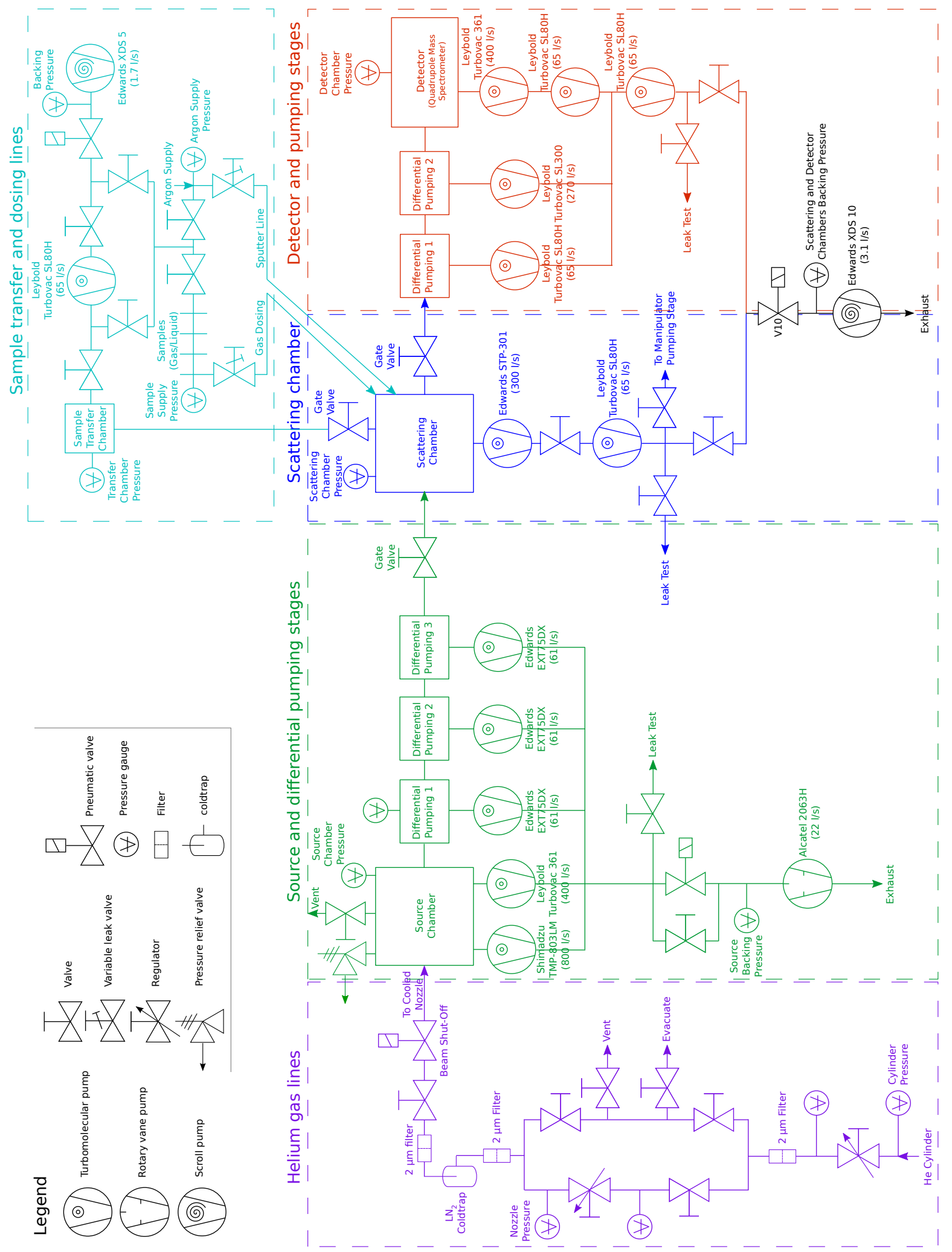

Figure 3.3: Schematic of MiniScat's vacuum system, including the make and pumping speed (in nominal $\mathrm{N}_{2}$ pumping speeds) of all pumps [48,49,51,52]. Vacuum chambers are drawn as rectangular boxes. The source arm components are shown in green, the scattering chamber in blue, the sample transfer system and gas dosing lines in cyan and the detector arm in red. Furthermore, a gas flow diagram of the helium beam source is included in violet. 
the laser through both viewports. The diffraction pattern remained unchanged confirming that the components do not move significantly under vacuum.

\subsubsection{Power distribution and computer control}

The electronic control system applied to the Cambridge HeSE spectrometer [53] has been adapted for MiniScat. The entire machine is run by a computer which reads from sensors, controls the main pieces of equipment, takes measurements and monitors the system status (e.g. lab temperature, pressure in the vacuum chambers, cooling water status, etc.). A power distribution unit supplies the power to all pumps and other electronic instruments. Computer control ensures that certain components can only be turned on in a safe environment - e.g. the source backing pump can only operate when cooling water supply is ensured - or that valves are not accidentally opened when the pressure on either side is too high.

An uninterruptible power supply (UPS) keeps the magnetically levitated turbomolecular pumps (source and scattering chamber pumps) and computer control facilities running for approximately 20 minutes in the case of a mains power failure, to avoid damage to the equipment and to maintain UHV conditions.

\subsubsection{Beam source}

The helium beam is created by supersonic expansion of pressurised helium gas through a $10 \mu \mathrm{m}$ nozzle into the source chamber where the centre portion of the expansion cone passes through a skimmer to form a directional beam. The beam energy is controlled through the nozzle temperature. The nozzle pressure used during experiment is limited by the capacity of the source pumps, and typically kept below 100 bar to avoid beam attenuation from excess background gas in the source chamber. Helium- 4 is used directly from a gas cylinder supplied at a pressure of 200 bar which can be regulated down to the desired nozzle pressure. The helium gas passes through several filters and a coldtrap filled with $1.5 \mathrm{~mm}$ diameter pellets of molecular sieves Alfa Aesar type 13X and type 4A (50\% each) to avoid blocking the nozzle with contaminants.

MiniScat was constructed at the same time as the new source for the HeSE spectrometer which is described in detail in Chapter 4. Therefore, the original setup of MiniScat was with the new source. A $0.5 \mathrm{~mm}$ model 2 Beam Dynamics nickel skimmer [54] is installed 
on a skimmer mount of the new design. After extensive testing, the new source was installed on the HeSE apparatus and the original HeSE source transferred onto MiniScat, yet the new skimmer mount was retained. The main difference between the two sources is that the new source can operate at nozzle temperatures as low as approximately $11 \mathrm{~K}$ (2.5 meV beam energy), whereas the original one does not typically reach temperatures much below $37 \mathrm{~K}(8.0 \mathrm{meV})$. While crucial for the resolution of the HeSE spectrometer, ultralow beam energies are not usually desirable on MiniScat as they are accompanied by reduced signal intensity and hence both sources provide approximately equivalent experimental results on MiniScat.

\subsubsection{Scattering chamber and sample preparation facilities}

MiniScat's scattering chamber is equipped with a range of sample preparation facilities. A sputter gun enables sample cleaning through argon ion sputtering. The sample can be cooled through a transfer tube using liquid nitrogen or liquid helium and heated radiatively with a tungsten filament and additionally via electron beam (e-beam) heating by applying a high voltage.

A leak valve connects gas dosing lines to the scattering chamber (cf. cyan section in Figure 3.3) and can be used for dosing liquid or gaseous samples by backfilling the chamber. If directional dosing or evaporation of a solid is desired, a motorised z-shift can be connected onto the port labelled "port for evaporator" in the top image in Figure 3.2 to attach an appropriate evaporator. Dosing is typically monitored using the specular helium reflectivity, hence directional dosing is achieved from a port located between the incident and outgoing beam line. A gate valve ensures that UHV conditions in the scattering chamber are maintained when the evaporator is exchanged. The z-shift is dimensioned to ensure compatibility of evaporators used on the HeSE spectrometer.

A quadrupole mass spectrometer located centrally between the two beam arms can be inserted into the chamber on a z-shift for thermal desorption studies. In the initial setup, a Pfeiffer QMG422 quadrupole mass spectrometer was installed [55], which was later replaced with a Hiden quadrupole mass spectrometer [56] (exchanging the mass spectrometer used as a detector with the one on the scattering chamber). 


\section{Sample manipulator and transfer mechanism}

The crystal is mounted on a six-axis manipulator analogous to the one on the HeSE spectrometer, which enables horizontal x/y-motion and vertical z-motion to position the sample in the scattering position, rotation around the vertical centre axis to change the scattering angle ( $\gamma$-rotation), tilting with respect to the plane of the incident and outgoing beam to align the sample perpendicular to the scattering plane ( $\beta$-rotation) and finally $\alpha$-rotation within the crystal plane to change the azimuthal orientation. Sample cooling is provided through a coldfinger which is a hollow tube that can be filled with liquid nitrogen or liquid helium through a transfer tube. Temperature control is achieved using a Eurotherm controller, reading the temperature measured by a chromel/alumel type $\mathrm{K}$ thermocouple connected to the sample mount and controlling the current through the filament to counter-heat the cooled sample.

Breaking the vacuum in the scattering chamber necessitates baking the chamber to restore UHV conditions. Being able to change samples in situ, without breaking the vacuum, hence reduces the time to change a crystal from approximately a week to only a few hours and extends the range of experiments which can be performed (e.g. comparative studies on a variety of different substrates). Therefore, a new sample transfer system for the HeSE spectrometer has been developed by Pepijn Kole [27], illustrated in Figure 3.4. It includes a transfer chamber which is connected to the scattering chamber through a gate valve (cf. cyan section in Figure 3.3). The sample is mounted on a sample cartridge which can be installed in the scattering chamber using two tool arms (visible in the top photograph in Figure 3.2). The transfer chamber can reach pressures in the $10^{-7}$ mbar to $10^{-8}$ mbar range which results in negligible contamination of the scattering chamber during the transfer process.

Initially, Pepijn Kole developed the sample transfer manipulator on MiniScat before it was moved onto the main instrument. When the new manipulator was installed on the main instrument, the tool arms remained on the scattering chamber and the original HeSE manipulator, a similar six-axis manipulator without sample transfer functionality, was installed on MiniScat. With the aim to make a faster experimental throughput on MiniScat possible and to improve the compatibility of the two instruments, the head of this manipulator was rebuilt as an exact copy of the HeSE sample transfer manipulator. Figure 3.5 shows photographs of the upgraded manipulator including an installed sample cartridge. Each sample can be mounted on its individual sample cartridge containing a filament for heating the crystal, a thermocouple and a high voltage connection. The 


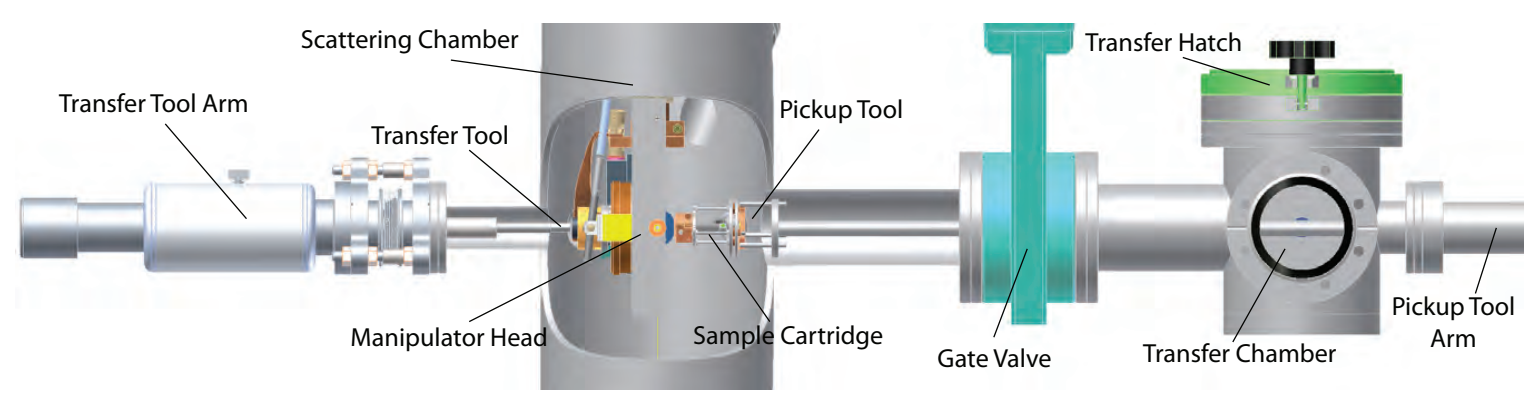

Figure 3.4: Illustration of the sample transfer system (from [27]). To install a new sample in the chamber, it is mounted on the sample cartridge and inserted into the transfer chamber which can be vented to atmosphere while the gate valve is closed. The transfer hatch is then closed and the transfer chamber evacuated before the gate valve can be opened. The sample cartridge is inserted into the scattering chamber using the pickup tool arm and locked onto the manipulator head with the transfer tool arm. The pickup tool can then be unlocked from the cartridge, both arms retracted and the gate valve closed. The reverse process is applied to remove the sample from the scattering chamber.

cartridge can be inserted in either the MiniScat or the HeSE scattering chamber as required, allowing much more efficient use of the two instruments.

The HeSe technique requires magnetic shielding from stray magnetic fields near the helium-3 beam. In order to make the MiniScat manipulator compatible with the HeSE instrument, all components have been constructed from non-magnetic materials (e.g. titanium was used instead of stainless steel). This ensures that both the components of the two arms as well as the manipulator could be used on the main machine, should the need arise.

\subsubsection{Detector}

A commercial quadrupole mass spectrometer tuned to mass 4 amu is used as helium detector. Measurements are taken by pulse counting and the count rate recorded using the control computer. Initially, the Hiden mass spectrometer was used, which was later replaced with the Pfeiffer mass spectrometer when the Hiden quadrupole was moved to the scattering chamber to improve thermal desorption experiments. 

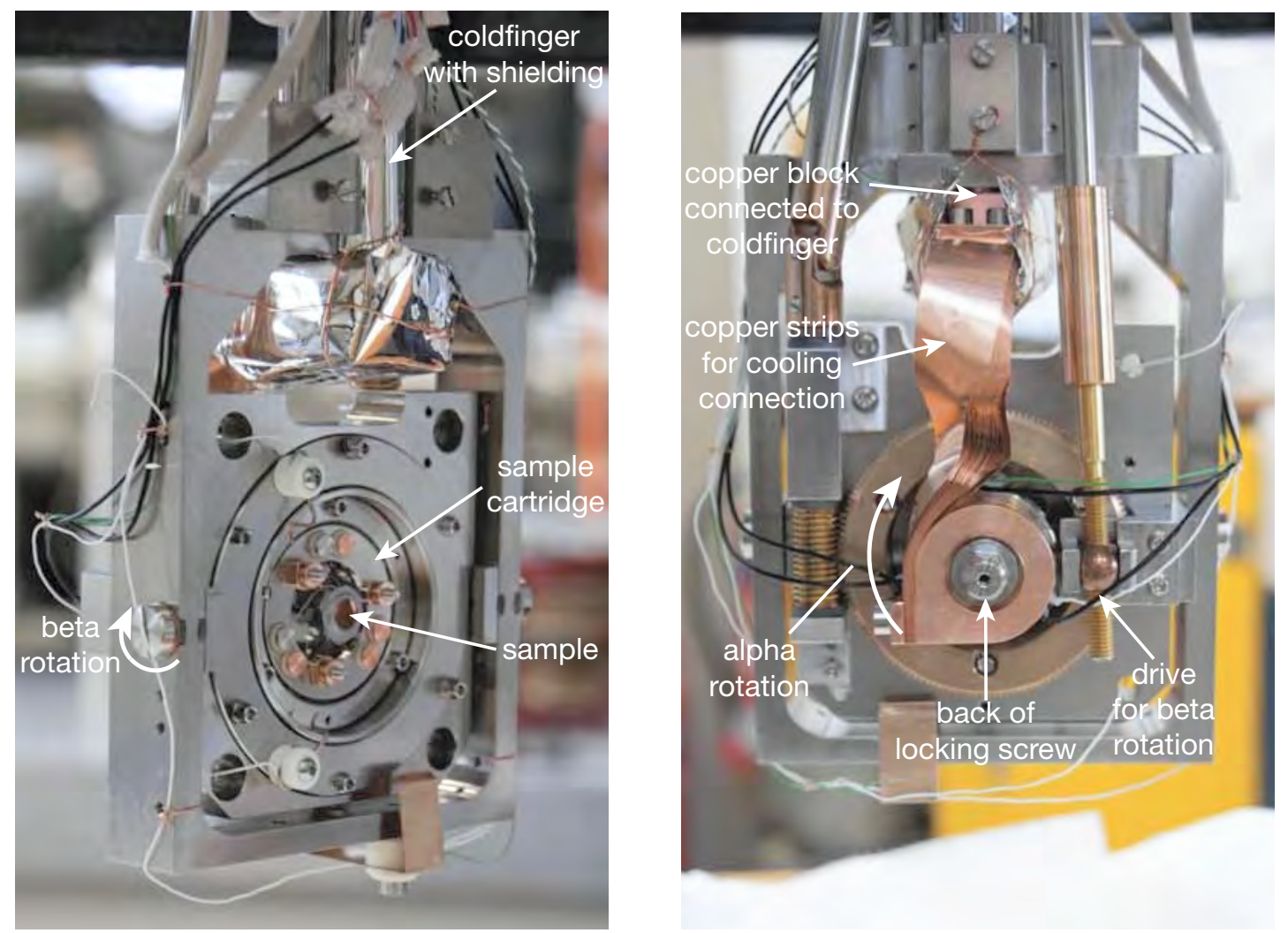

Figure 3.5: MiniScat's sample transfer manipulator head, photographed ex situ. Annotations highlight the main components.

\subsubsection{Two different instrument configurations}

As described above, MiniScat has been used in two main configurations, using different components. The "original setup" has been used for initial testing as well as the experiments described in Chapters 6 and 7, while improvements to the setup have been made subsequently, leading to the "improved setup". The main change in functionality is that the mass spectrometer in the scattering chamber can be moved more closely to the sample surface in the improved setup, allowing for quantitative TDS measurements. Table 3.2 summmarises the two configurations.

\subsection{Characterisation}

At the time of writing, MiniScat has been in routine use for approximately two years and has proven beneficial for sample preparation and characterisation as well as for structural 


\begin{tabular}{lll}
\hline Component & Original setup & Improved setup \\
\hline Source & New source (Ch. 4) & Old HeSE source \\
Mass spectrometer for TDS & Pfeiffer QMG422 & Hiden Quadrupole \\
Detector & Hiden Quadrupole & Pfeiffer QMG422 \\
\hline
\end{tabular}

Table 3.2: Comparison of the original and improved experimental setups of MiniScat.

studies $[27,57,58]$. This section summarises initial measurements carried out for testing and characterisation purposes, as well as further results to illustrate the versatility of the instrument.

\subsubsection{Uptake measurements}

Monitoring the specular helium reflectivity can provide information about interactions amongst adsorbates, structure formation, phase changes and adsorbate coverage [15] and is thus one of the main experimental features of MiniScat.

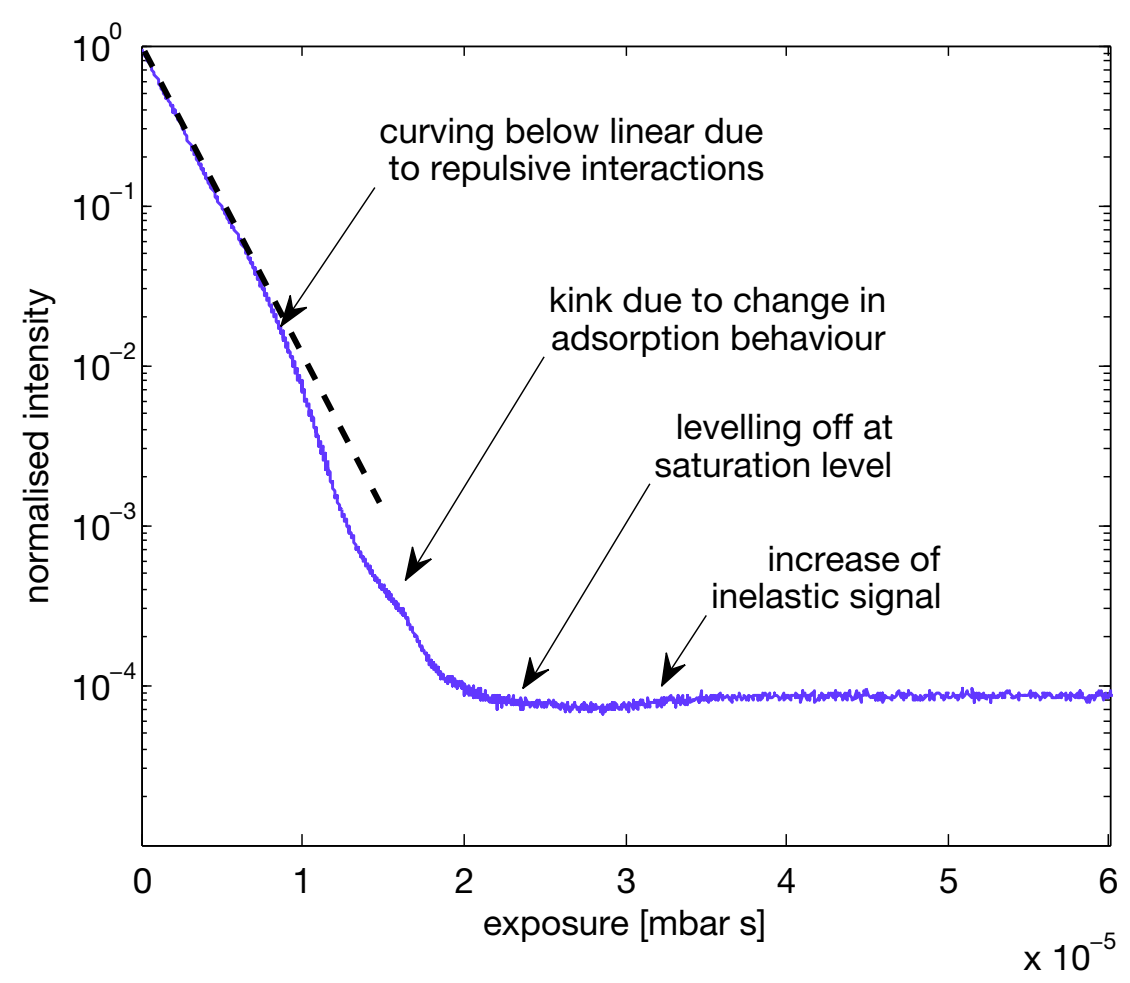

Figure 3.6: Uptake curve for thiophene $/ \mathrm{Cu}(111)$ at $100 \mathrm{~K}$ measured on MiniScat (original setup). 
Figure 3.6 presents an uptake curve for thiophene on $\mathrm{Cu}(111)$ at $100 \mathrm{~K}$ (cf. Chapter 7), measured on MiniScat. Increasing the emission current of the detector after an exposure of $1.1 \cdot 10^{-5}$ mbar s ensures that the noise level remains low despite a drop in the helium intensity by more than four orders of magnitude. The presented uptake curve illustrates a variety of features which can be observed in uptake curves (cf. Section 2.1), such as a deviation from a linear curve due to lateral adsorbate interactions [15], a kink in the curve indicating a phase change on the surface and levelling off when saturation is reached on the surface.

\subsubsection{Diffraction studies}

By scanning a range of scattering angles, diffraction measurements can be performed, giving information about periodic features on the surface. Figure 3.7 shows the first diffraction experiments that were performed on MiniScat (original setup) of a $\mathrm{LiF}(100)$ surface. Since MiniScat is a fixed angle instrument, diffraction scans are intrinsically asymmetric, as explained in Section 2.1. Note that in this particular example the peaks are widened due to steps on the LiF surface. The peak positions can be used to determine the total scattering angle of the instrument from the known lattice geometry of LiF [59].

As defined in Section 2.1, the diffraction peak position in momentum transfer space can be calculated from the scattering angles via

$$
\Delta K=k_{i} \cdot\left[\sin \left(\frac{1}{2} \Theta_{\text {total }}-\Theta_{\text {offspec }}\right)-\sin \left(\frac{1}{2} \Theta_{\text {total }}+\Theta_{\text {offspec }}\right)\right],
$$

where $\Theta_{\text {total }}$ is the total scattering angle of the instrument, $\Theta_{\text {offspec }}$ the angular off-specular position of the diffraction peak and $k_{i}$ the incident wave vector, which is given through

$$
k_{i}=\frac{\sqrt{2 m E_{i}}}{\hbar}
$$

with $\hbar$ the reduced Planck constant and $m$ the mass of the helium atom. For a monatomic gas, the mean energy of the incident helium beam, $E_{i}$, can be calculated from the nozzle temperature, $T$, as [60]

$$
E_{i}=\frac{5}{2} k_{B} T
$$



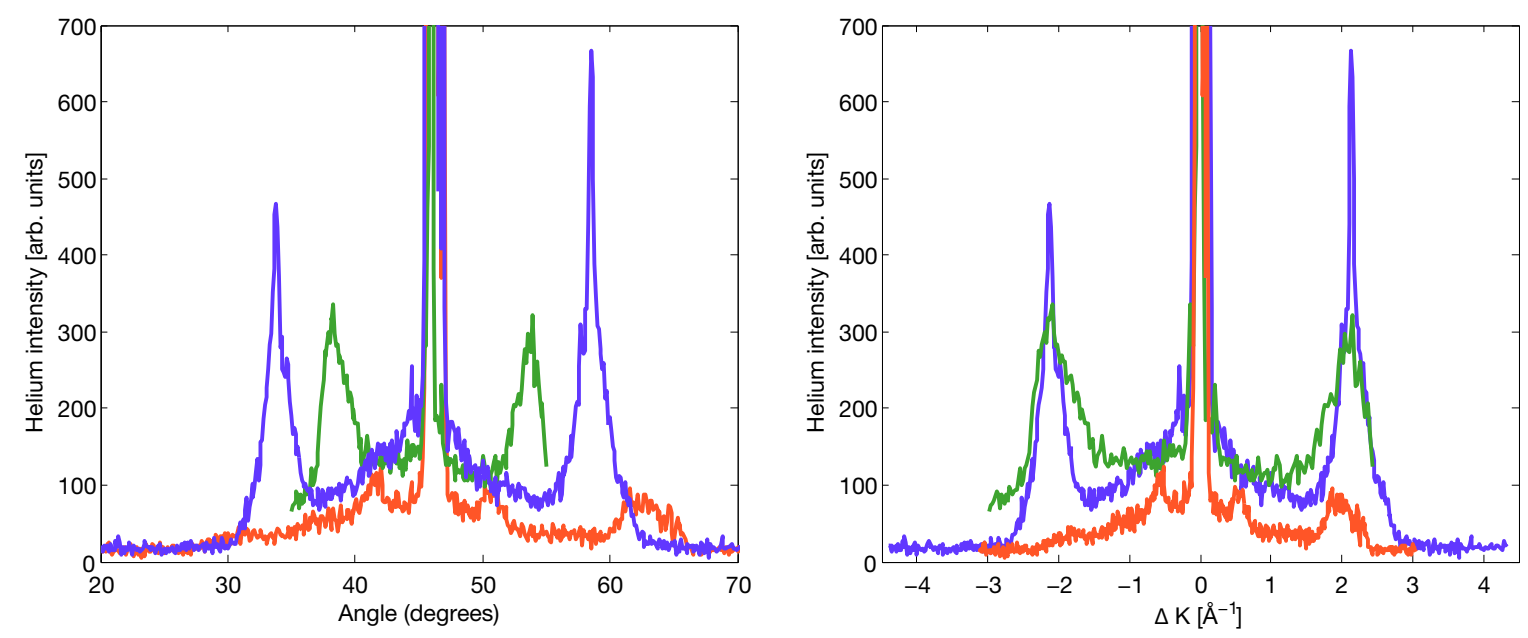

Figure 3.7: First diffraction experiments performed on MiniScat to determine the instrument angle. The measurements show diffraction peaks of a LiF surface for three different beam energies. The green, blue and red curves were recorded using a $300 \mathrm{~K}$, $120 \mathrm{~K}$ and $60 \mathrm{~K}$ nozzle temperature, respectively, corresponding to a beam energy of $64.6 \mathrm{meV}, 7.0 \mathrm{meV}$ and $5.0 \mathrm{meV}$. The left-hand panel shows the diffraction scans as a function of scattering angle while the panel on the right presents the same data after conversion of the angle to momentum transfer, illustrating that the same diffraction peaks occur at different scattering angles for different beam energies.

where $k_{B}$ is the Boltzmann constant. The surface lattice constant of $\operatorname{LiF}(100)$ is $a=2.85 \AA$, which corresponds to a diffraction peak position of $\Delta K=2 \pi / a=2.21 \AA^{-1}$. Re-arranging Equation 3.1 gives the total scattering angle,

$$
\Theta_{\text {total }}=2 \cdot \cos ^{-1}\left(-\frac{\Delta K}{2 k_{i} \sin \Theta_{\text {offspec }}}\right),
$$

defined by the substrate lattice through $\Delta K$ and the wave vector of the incident beam.

On the new source, the nozzle temperature is measured accurately using a silicon diode $^{1}$. The peak positions in the left-hand panel show an off-specular position of $8.0 \pm 0.1^{\circ}$ using a $300 \mathrm{~K}$ nozzle and $12.8 \pm 0.1^{\circ}$ for the measurement with a $120 \mathrm{~K}$ beam. The peaks using a $60 \mathrm{~K}$ nozzle temperature are fairly weak and thus cannot be interpreted accurately. Using Equation 3.4, the total scattering angle of MiniScat can be calculated as $90 \pm 2^{\circ}$ using the data for a $300 \mathrm{~K}$ nozzle, and $90 \pm 1^{\circ}$ from the $120 \mathrm{~K}$ nozzle data.

${ }^{1}$ After the source has been installed on the HeSE spectrometer, experiments recording the beam profile using the ingoing solenoid have confirmed that the temperature readings are accurate to within $1 \mathrm{~K}$ in the temperature range used for these measurements (cf. Section 4.2.2). 
Subsequently, diffraction studies on a range of other systems with known diffraction peak positions have been performed, confirming the instrument angle as $90^{\circ}[27,57,58]$.

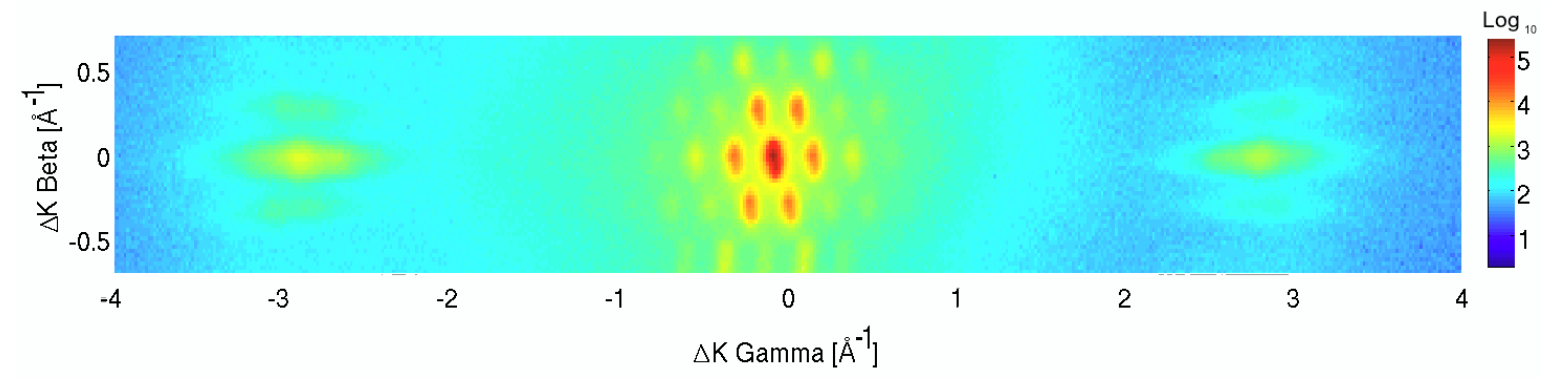

Figure 3.8: 2D diffraction of graphene/ $\mathrm{Ru}(0001)$ at $125 \mathrm{~K}$ measured on MiniScat using a beam energy of $25.9 \mathrm{meV}$ (from [57]). Peaks show a Moiré lattice in addition to the carbon lattice.

In addition to one-dimensional diffraction scans, two-dimensional studies can be performed using the $\beta$-rotation axis (i.e. tilt around the horizontal axis of the crystal) in addition to the $\gamma$-rotation, thus scanning a two-dimensional momentum transfer space. Figure 3.8 shows such a 2D diffraction pattern recorded on MiniScat for graphene/ $\mathrm{Ru}(0001)$, which exhibits diffraction peaks from graphene as well as additional peaks closer to specular (centre portion of the Figure) caused by the Moiré lattice formed by graphene on $\mathrm{Ru}(0001)$ [57].

\subsubsection{Thermal desorption spectroscopy}

The third type of experiment which is typically performed on MiniScat for adsorption studies is thermal desorption spectroscopy (TDS). As mentioned earlier, MiniScat is configured to allow simultanous monitoring of the specular helium reflectivity and the signal in a mass spectrometer during thermal desorption, thus providing information about the desorbing species as well as changes in the degree of order on the surface.

During TDS experiments with the Pfeiffer mass spectrometer (original setup), the sample-mass spectrometer distance was limited to a minimum of approximately $100 \mathrm{~mm}$ (due to the dimensions of the mass spectrometer and the scattering chamber), while the Hiden mass spectrometer can approach the sample more closely, to approximately $5 \mathrm{~mm}$ (improved setup), making quantitative TDS studies possible. Two example TDS measurements using the two different experimental configurations are presented in Figure 3.9. 

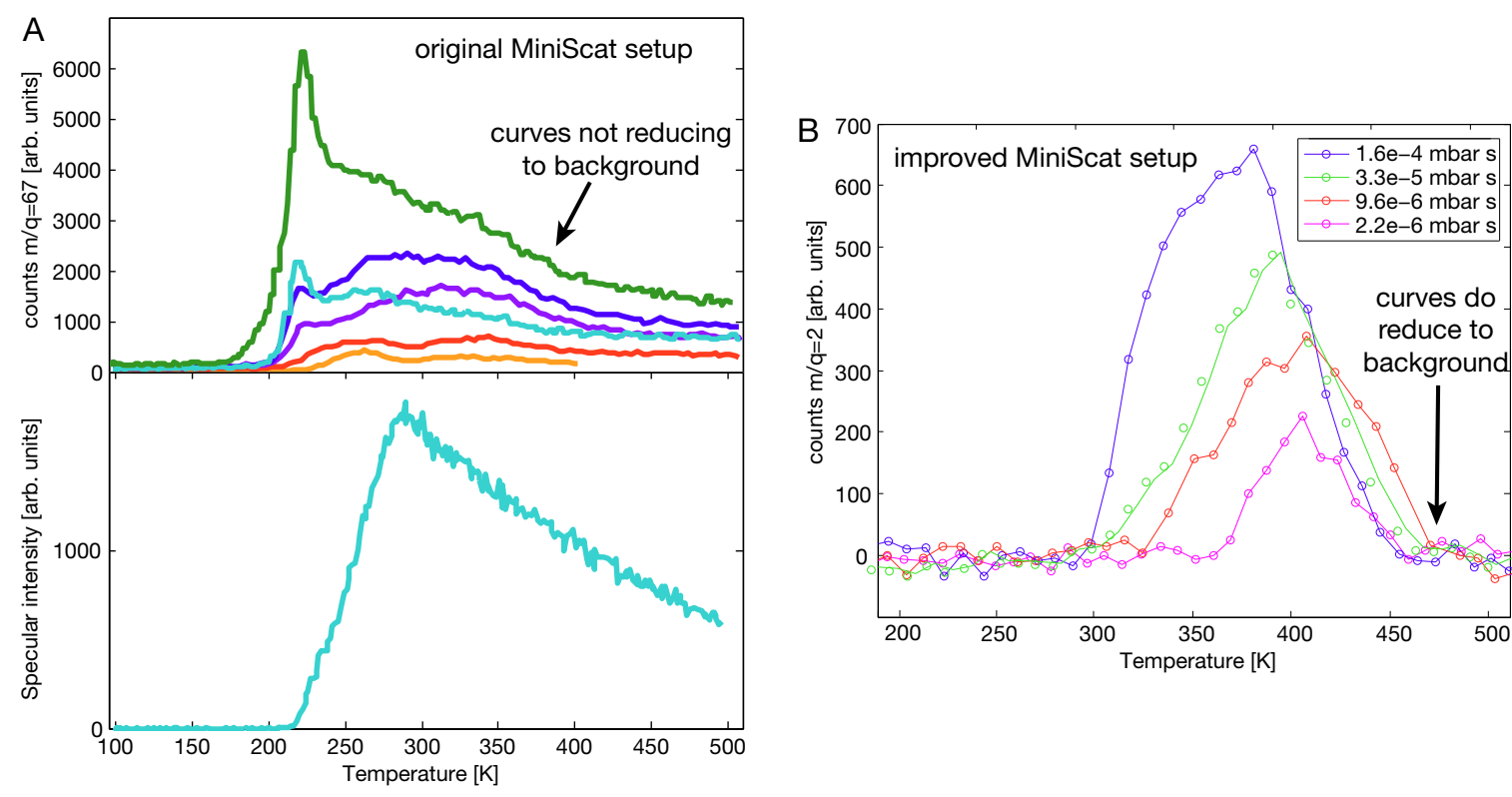

Figure 3.9: Thermal desorption spectra recorded on MiniScat. (A) shows a desorption experiment using the original setup for several sub-monolayer coverages of pyrrole/ $\mathrm{Cu}(111)$ at a heating rate of $3 \mathrm{~K} / \mathrm{s}$ (cf. Chapter 6). Desorption is monitored with a mass spectrometer (top) and the specular helium signal (bottom) simultaneously. A peak in the helium signal implies that desorption is complete at $290 \mathrm{~K}$. The signal in the desorption spectra, however, does not reduce to the background level at this temperature, most likely due to desorption from sample mount and manipulator that is picked up by the mass spectrometer. (B) presents desorption measurements using the improved setup and a heating rate between $2 \mathrm{~K} / \mathrm{s}$ and $3 \mathrm{~K} / \mathrm{s}$, studying the desorption of molecular hydrogen from a Ni(111) surface at coverages below $1 \mathrm{ML}$ (from [58]). The peaks show a sharp edge as the signal drops back to the background level since the mass spectrometer in the improved setup records predominantly desorption from the crystal.

The bottom left panel shows the evolution of the specular helium signal during heating at a rate of $3 \mathrm{~K} / \mathrm{s}$ for a submonolayer coverage of pyrrole/ $\mathrm{Cu}(111)$ (cf. also Chapter 6). As desorption progresses, the order on the surface and therefore the sample reflectivity increase. The signal levels off at $290 \mathrm{~K}$ when all pyrrole is desorbed and the clean $\mathrm{Cu}(111)$ surface recovered. The maximum signal level in this experiment was comparable to that for the clean crystal prior to dosing, confirming that desorption is complete at $290 \mathrm{~K}$. The decrease in the signal after the peak can be due either to Debye Waller attenuation with increasing temperature or a small misalignment of the sample from the large change in temperature from $100 \mathrm{~K}$ to $500 \mathrm{~K}$, and thus a reduction in helium signal at higher temperatures. In the top panel of Figure 3.9 (A), desorption of pyrrole from a $\mathrm{Cu}(111)$ surface is monitored in the mass spectrometer, using the original setup. It is apparent 
that the curves do not reduce to the background level when the helium reflectivity implies that desorption from the crystal is complete. The shoulder at the end of each curve is caused by desorption from the sample mount and manipulator, making it difficult to analyse these spectra quantitatively.

Figure 3.9 (B) shows a different desorption experiment, for $\mathrm{H}_{2} / \mathrm{Ni}(111)$, using the improved setup. Due to its proximity to the sample, the mass spectrometer predominantly records species desorbed from the crystal and thus the peaks level off after desorption is completed, facilitating integration of the peaks and coverage calibration from TDS measurements. Furthermore, since hydrogen is one of the main contaminants in a vacuum chamber, these measurements demonstrate the high sensitivity of the TDS experiment on MiniScat.

\subsection{Conclusions}

The design and characterisation of MiniScat, a new small helium atom scattering apparatus, were presented in this chapter. The motivation for this new piece of equipment was fourfold. First, the investigation of more complex adsorbate systems by HeSE creates the necessity for more detailed preliminary studies of the adsorbate behaviour of these systems through diffraction and uptake experiments, which can now be performed on MiniScat prior to the dynamics measurements. Second, MiniScat can be used to perform experiments which cannot be carried out on the HeSE spectrometer, such as thermal desorption spectroscopy. Third, the apparatus is compatible with the main instrument and can thus be used for equipment development purposes such as that of the new source (cf. Chapter 4) and a sample transfer manipulator [27] for the HeSE spectrometer. Lastly, MiniScat can be used for scattering experiments using different probes, such as neon or argon. Heavy atom scattering provides accurate information about the level of surface corrugation and can be sensitive to structural details invisible to helium atoms.

MiniScat is a $90^{\circ}$ fixed angle scattering machine. The helium beam is created by a high-intensity supersonic beam source while a commercial quadrupole mass spectrometer is used as a helium detector. The sample is mounted on a six-axis manipulator which defines and changes the scattering angle and azimuthal direction of the crystal. The scattering chamber further contains facilities for sample preparation, covering a wide range of adsorbates. A sample transfer system allows rapid changing of the sample without breaking the vacuum in the scattering chamber, and ensures optimum compatibility with 
the HeSE spectrometer as sample cartridges can be used on both instruments. At the time of writing, MiniScat has been in routine use for two years, providing valuable structural information for a range of adsorbate systems (cf. Chapters 6 and 7 and $[27,57,58]$ ) before dynamics measurements were performed. 


\section{Chapter 4}

\section{An improved high intensity supersonic helium beam source}

The helium beam source is one of the crucial components of the helium-3 spin-echo (HeSE) spectrometer and determines the energy resolution of the experiment. The Cambridge instrument is designed to operate at a beam energy of $8 \mathrm{meV}$, where the magnetic fields provide optimal focussing and thus maximum signal intensity, giving a nominal energy resolution of $3 \mu \mathrm{eV}$. During standard operation, the main requirement of the HeSE source is to provide a stable $8 \mathrm{meV}$ helium-3 beam with high signal intensity to minimise measurement time, allowing investigation of surface motion on timescales between $0.01 \mathrm{ps}$ and $680 \mathrm{ps}$. While the original beam source for the HeSE spectrometer was designed to create an $8 \mathrm{meV}$ helium beam, such low beam energies often could not be maintained and experiments were most commonly performed using an energy between $9.5 \mathrm{meV}$ and $11.5 \mathrm{meV}[28,31,61]$.

In general, the larger an adsorbate, the slower and more complex its diffusion behaviour $[1,5,62]$. The aim of this thesis to extend the application of HeSE to more complex adsorbate systems therefore creates the need to increase the accessible timescales into the nanosecond range and makes it desirable to perform HeSE experiments at lower beam energies, despite the resulting loss in signal intensity from overfocussing the beam in the magnetic fields.

This chapter presents a new high intensity supersonic helium beam source for the Cambridge HeSE spectrometer, consisting of a new nozzle assembly and a new skimmer mount, which not only allows routine operation at a beam energy of $8 \mathrm{meV}$, but also reaches energies as low as $2.5 \mathrm{meV}$, increasing the energy resolution of the experiment by 
a factor of 5.5. This upgrade opens up a whole new measurement range, extending the accessible timescales to $3.8 \mathrm{~ns}$. After a brief introduction to supersonic beam sources, this chapter describes the design of the new nozzle assembly and demonstrates the performance of the new source. Furthermore, an improved skimmer mount design is presented, which increases the signal intensity by $20 \%$. It should be noted that the general design idea for the new source was formulated by Andrew Jardine.

\subsection{Introduction}

The use of a nozzle source to create supersonic atomic and molecular beams of high monochromaticity was first suggested by Kantrowitz and Grey in 1951 [63]. In a nozzle, or free-jet, source a supersonic beam is created by expansion through a pinhole nozzle and selecting the centre section of the expansion cone by a skimmer [60]. Figure 4.1 shows a schematic illustration of the expansion process. High pressure gas (typically between 10 bar and 200 bar) expands through a nozzle, on the order of $5 \mu \mathrm{m}$ to $20 \mu \mathrm{m}$ in diameter, into a vacuum chamber and forms an expansion cone. Due to the pressure difference inside the nozzle and in the vacuum chamber, the velocity of the atoms or molecules increases as they move through the nozzle and can reach sonic speed at the exit, which corresponds to a Mach number, $M$, of 1 . In the vacuum chamber, subsequent expansion occurs. The gas first expands isentropically, forming a so-called "zone of silence" where $M>>1$, and is then compressed in the Mach disk and barrel shock zones. The region of transition from continuum flow to free molecular flow, where the last collisions occur, is termed "quitting surface".

The beam is created by selecting the centreline velocities of the expansion cone using a skimmer which is typically placed in the region of free molecular flow. The precise shape of the expansion cone is dependent on the pressure in the vacuum chamber and thus on the size of the pumps, as described by Campargue [64]. When pressures are kept low (in the $10^{-6}$ mbar to $10^{-3}$ mbar region), using high capacity pumps such as diffusion pumps, the formation of shock fronts and a zone of silence is limited and the expansion process essentially finished before the Mach disk shock. The skimmer can thus be placed in a region where the expansion process is complete and minimal interference of the gas with the skimmer walls occurs (Fenn type source). When lower capacity pumps and higher chamber pressures (typically $10^{-2}$ mbar to 1 mbar) are used, such as in a Campargue type source, stronger shock-wave structures are formed and the skimmer 


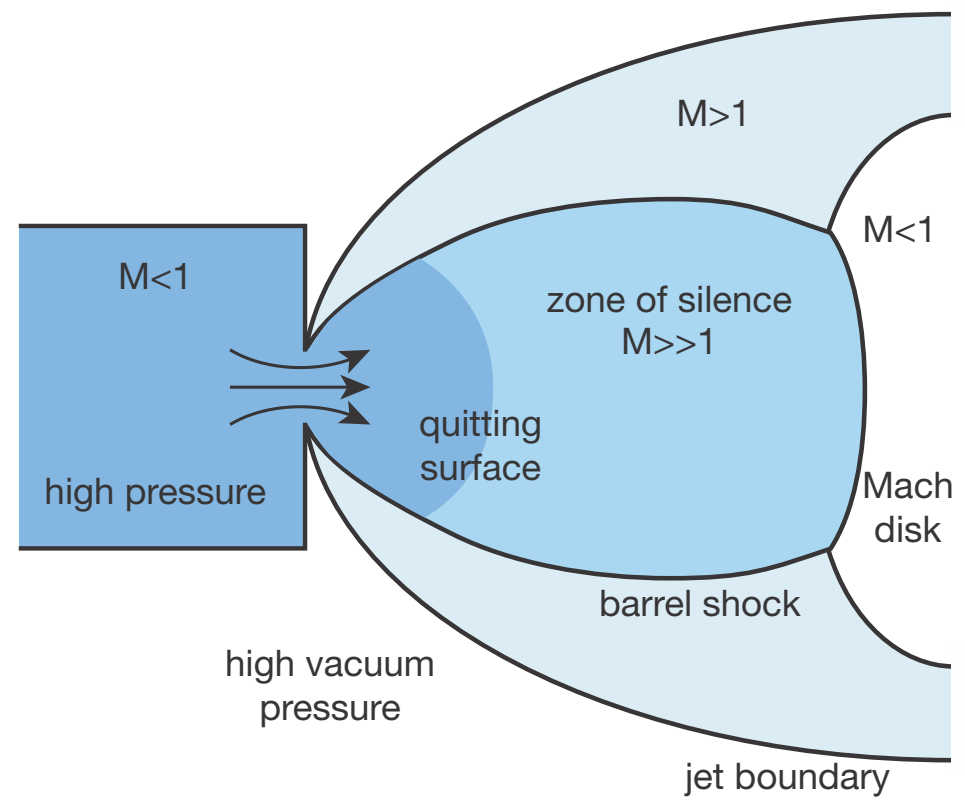

Figure 4.1: Schematic illustration of the expansion process in a supersonic helium beam source. High pressure gas is expanded into a vacuum chamber through a small nozzle and forms a supersonic expansion cone. The central section where the Mach number, $M,>>1$ is an isentropic region, termed "zone of silence". The zone of silence is surrounded by the Mach disk and barrel shock. The transition region from continuum to free molecular flow is known as the "quitting surface".

needs to be positioned in the zone of silence. In a Campargue type source, the expansion process thus continues beyond the skimmer [64].

The beam energy, $E_{0}$ is determined by the temperature of the nozzle, $T_{\text {nozzle }}$, through the relationship

$$
E_{0}=\frac{5}{2} k_{B} T_{\text {nozzle }}
$$

where $k_{B}$ is the Boltzmann constant. A beam energy of $8.0 \mathrm{meV}$ is achieved by expansion through a nozzle at $37 \mathrm{~K}$. In order to create a beam of constant energy, the nozzle is connected to a cooling and counter-heating facility. As shown in Equation 2.9, the spin-echo time, $t$ is inversely proportional to $v_{0}^{3}$, which implies that the resolution of the HeSE spectrometer (i.e. the maximum $t$ ) is inversely proportional to $E_{0}^{3 / 2}$. In order to improve the energy resolution, the operating temperature of the nozzle therefore needs to be reduced. All components involved in the positioning and temperature control of the nozzle are referred to as the "nozzle assembly". The nozzle assembly of the new 
beam source includes improved thermal shielding enabling routine operation at nozzle temperatures as low as $11.8 \mathrm{~K}$.

While lowering the beam energy provides the desired improvement in resolution, the beam is de-/overfocussed in the magnetic fields of the HeSE spectrometer when $E_{0}$ deviates from the nominal $8 \mathrm{meV}$. It is therefore desirable to improve the beam intensity to ensure sufficient signal even at low beam energies. In general, the beam intensity increases with the pressure behind the nozzle, or for short "nozzle pressure". When using the original HeSE source at low nozzle temperatures, however, beam attenuation has been observed as the flow through the nozzle was increased beyond a certain point, which resulted in a peak in the beam intensity at intermediate pressures [24]. It has been suggested that backscattering of the beam in the nozzle-skimmer region is likely to be the main source of attenuation [24]. Therefore, an improved skimmer mount has been designed for the new source, moving the skimmer further away from the chamber wall and reducing the size of components in the immediate proximity of the skimmer in an attempt to increase the signal intensity.

\subsection{A new low temperature nozzle assembly}

The original source of the HeSE spectrometer is a standard supersonic atomic beam source. It was installed on the top flange of the source chamber on a custom-made $\mathrm{x}, \mathrm{y}, \mathrm{z}$-stage to allow precise positioning of the source in front of the skimmer [23]. The nozzle assembly thus had to be designed at a $90^{\circ}$ angle with flexible copper braids providing the cold path. The nozzle assembly was wrapped in super-insulation foil to shield it from radiation from the background gas [53].

The new nozzle assembly uses a $180^{\circ}$ design instead. The main advantages of the new, straight nozzle assembly are a cooling path consisting of solid-copper components instead of copper braids, and a copper tube around the nozzle and gas supply tube, providing much improved heat shielding compared to the previous design which relied purely on super-insulation foil. Therefore, the new source can operate at much lower nozzle temperatures, down to approximately $11 \mathrm{~K}$. 


\subsubsection{Design}

In the new nozzle assembly, a McAllister x,y,z-manipulator [47] is installed on a sideways port of the source chamber, as shown schematically in Figure $4.2(\mathrm{~A})$. The manipulator is fitted with springs to compensate for its own weight and that of the nozzle assembly.

A

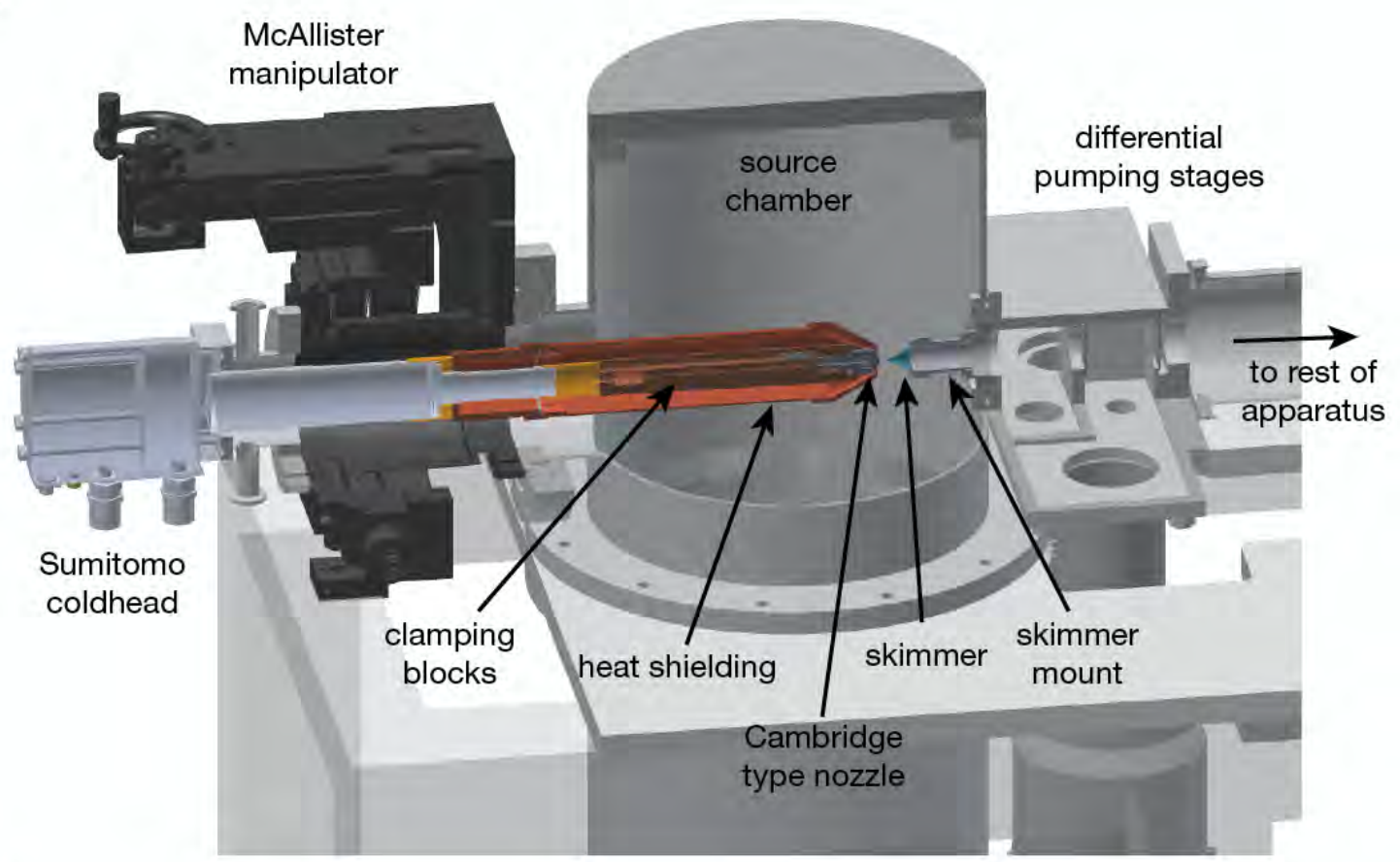

B

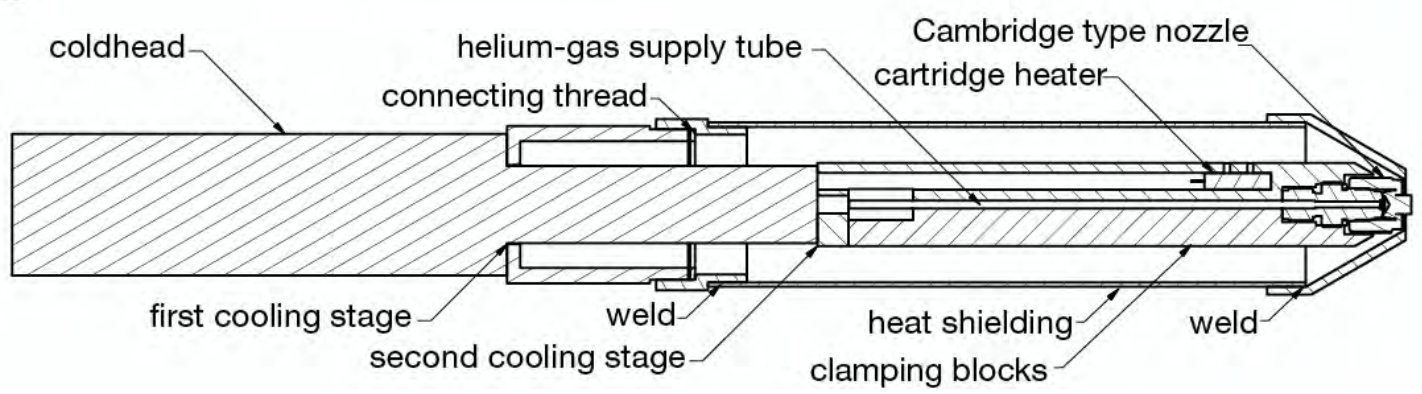

Figure 4.2: Autodesk Inventor drawings of the new source. (A) shows a $3 \mathrm{D}$ view of the source chamber, cut through the centre to uncover the components of the nozzle assembly and skimmer mount. Drawings for the manipulator and coldhead have been provided by McAllister [47] and Sumitomo [65], respectively. (B) is a detailed cross-sectional drawing view of the new nozzle assembly including the copper clamping blocks, heat shielding and nozzle, mounted on the two cooling stages of the expander head. 
Nozzle cooling is provided by a $10 \mathrm{~K}$ coldhead (Sumitomo model DE-204SL expander head and model HC-4E compressor [65]), onto which the nozzle and heat shielding are mounted. Figure 4.2 (B) shows a schematic cross section of the new source. The nozzle itself is a Cambridge type nozzle design [66] with a $10 \mu \mathrm{m}$ diameter aperture, connected to the helium-3 recycling system [25] by an 1/8 inch stainless steel tube. Two copper blocks are mounted onto the second cooling stage of the expander head which clamp the helium gas supply tube and the nozzle, providing the cold path between expander head and nozzle. In addition, the tube winds around the second stage of the cryocooler to pre-cool the gas (not shown).

A Lakeshore silicon diode [67] mounted at the end of the copper blocks monitors the nozzle temperature, which is regulated by a Lakeshore temperature controller [67], reading from the silicon diode and stabilising the temperature by counter-heating with a cartridge heater installed inside one of the copper blocks. Several type K thermocouple wires allow measurement of the temperature at the two cooling stages of the expander head. To maximise the thermal contact between the different components, a thin layer of Apiezon N cryogenic high vacuum grease [68] is applied at their interfaces. All electrical wires are looped around each cooling stage at least 10 times to minimise measurement errors from a steep temperature gradient.

The nozzle and all components connecting it to the second expander head stage, as well as the helium gas supply tube, are contained within a copper tube with conical end to provide heat shielding from the radiation of the background gas. The thermal conductivity of the vacuum can be estimated by calculating the flux of particles per unit area,

$$
\frac{N}{A}=\frac{1}{4} n \bar{v}
$$

where $n$ is the number of particles per unit volume, $n=N / V$, or equivalently $n=p / k T$, and $\bar{v}$ is the mean particle velocity defined by $\bar{v}=\sqrt{\frac{8 k_{B} T}{\pi m}}$, with $k_{B}$ the Boltzmann constant, $T$ the particle's temperature and $m$ its mass. Rearranging Equation 4.2 provides an estimate of the heat flow from the background gas,

$$
\dot{Q}=p_{0} \sqrt{\frac{2 k_{B}}{T_{0} \pi m}} \Delta T,
$$

which depends on the pressure, $p_{0}$, and temperature, $T_{0}$, in the vacuum chamber, as well as the temperature difference between vacuum and nozzle, $\Delta T$. Without heat shielding, 
the thermal load from a vacuum of approximately $1 \cdot 10^{-3}$ mbar would be $70 \mathrm{~W} / \mathrm{m}^{2}$ for a nominal nozzle temperature of $10 \mathrm{~K}$, corresponding to black body radiation at $190 \mathrm{~K}$.

The heat shielding tube is constructed in two parts. The first component is fixed onto the first stage of the expander head, which provides cooling to approximately $77 \mathrm{~K}$, while the others are welded together and screwed onto the first part. The front of the heat shielding encloses the nozzle tightly without touching it. To allow for fabrication imprecision in the length of the clamping blocks and the heat shielding tube, the length of the copper tube can be fine-adjusted using spacers in the connecting thread of the two parts of the heat shielding. In contrast to the previous source where the angled nozzle assembly made effective heat shielding difficult, the gas supply tube in the new source is shielded by solid copper right up to the nozzle. Since the heat shielding tube is pre-cooled by the first stage of the coldhead, the thermal load is reduced from $70 \mathrm{~W} / \mathrm{m}^{2}$ to $16 \mathrm{~W} / \mathrm{m}^{2}$ by the copper tube alone. In addition, several layers of super-insulation foil between the heat shielding tube and the two clamping blocks as well as around the outside of the heat shielding tube further improve the thermal properties of the source significantly.

Another source of thermal load on the nozzle is the helium gas itself, which needs to be cooled from $300 \mathrm{~K}$ to the nozzle temperature, nominally $\geq 10 \mathrm{~K}$. At a typical flow rate of $r=10 \mathrm{mbar} 1 \mathrm{~s}^{-1}$, the resulting heat flow is

$$
\dot{Q}=r \cdot c_{p} \cdot \Delta T
$$

where $c_{p}=\frac{5}{2} R$ is the heat capacity and $\Delta T$ is the temperature difference between the gas inlet and the nozzle. By pre-cooling the helium gas to $77 \mathrm{~K}$ by looping the gas supply tube around the first stage of the expander head several times, the thermal load of the nozzle can be reduced by a factor of 5 , thus making the effect negligible $(<0.5 \mathrm{~W})$. In addition, all copper components have been polished to reduce their emissivity and thereby improve their thermal properties.

The completed source installed on the HeSE spectrometer is illustrated in several photographs in Figure 4.3, where (A) shows the coldhead and x,y,z-manipulator mounted on the source chamber, (B) gives a view from within the vacuum chamber, showing the heat shielding tube wrapped in super-insulation foil and the skimmer in its new mount (cf. Section 4.3), and (C) presents the interior of the nozzle assembly with the gas supply tube and the nozzle inserted into the recess of one of the two copper clamping blocks. 

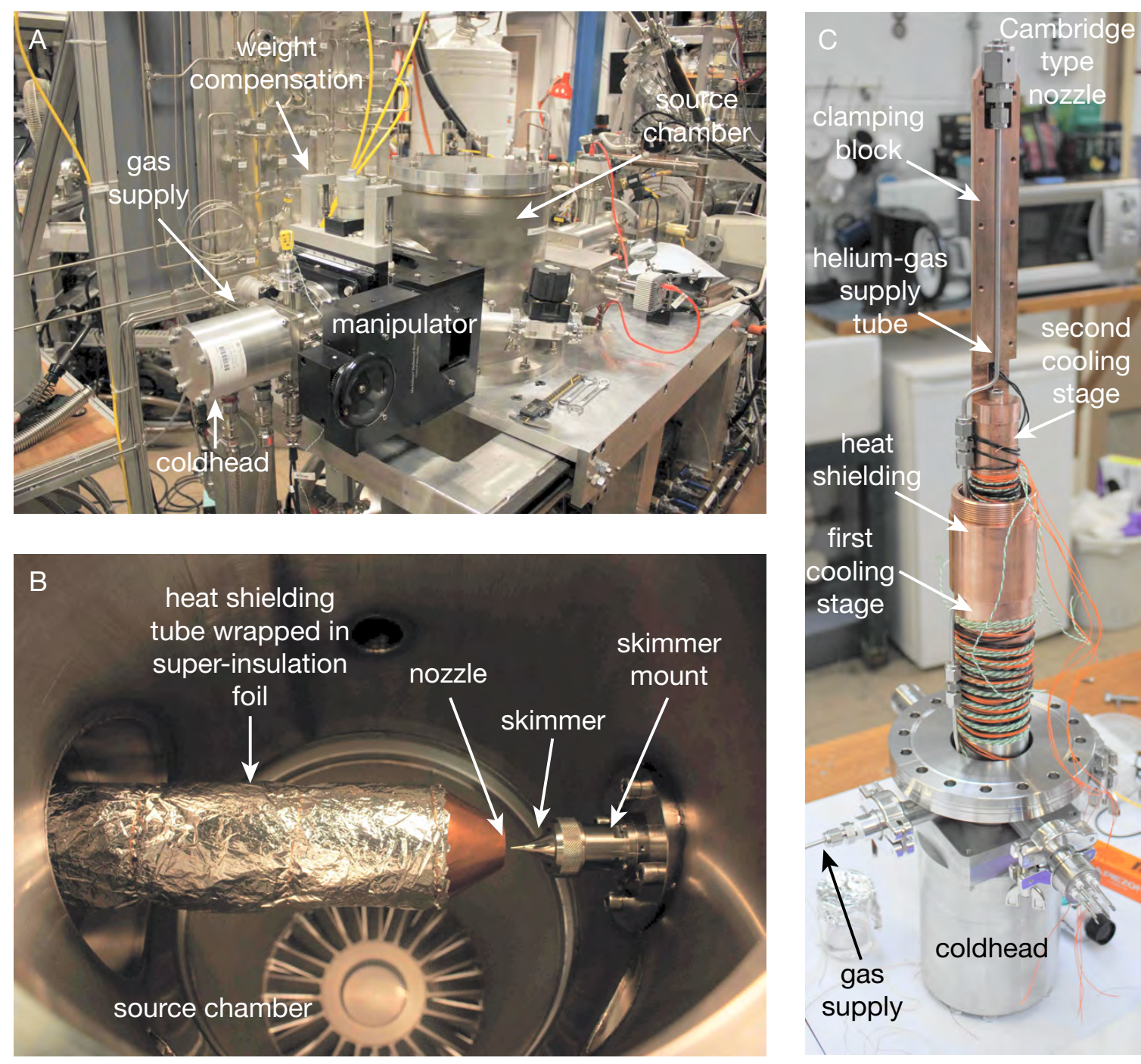

Figure 4.3: Photographs of the new helium beam source. (A) shows the source installed on the source chamber of the HeSE spectrometer, while (B) provides a view from within the source chamber, showing nozzle assembly and a skimmer in the new skimmer mount. (C) presents the interior of the nozzle assembly, where the helium supply tube and the nozzle assembly are inserted into a recess of one of the two clamping blocks and only the bottom part of the heat shielding tube is installed. Annotations illustrate the main components.

\subsubsection{Characterisation}

In an initial test, the nozzle was set to cool without counter-heating from the cartridge heater to investigate the lowest achievable temperature. The nozzle pressure was reduced to below 2 bar to minimise the thermal load while keeping a small gas flow to prevent 
ice formation blocking the nozzle ${ }^{1}$. Figure 4.4 presents the resulting cooling curve, where the temperature is as read from the silicon diode. After just over two hours, cooling from room temperature, the nozzle temperature levels off at a nominal temperature of $8.0 \mathrm{~K}$. After initial cooling - once the entire nozzle assembly is cold - the nozzle temperature can be stabilised at any particular temperature in a range up to approximately $50 \mathrm{~K}$ within less than 10 minutes.

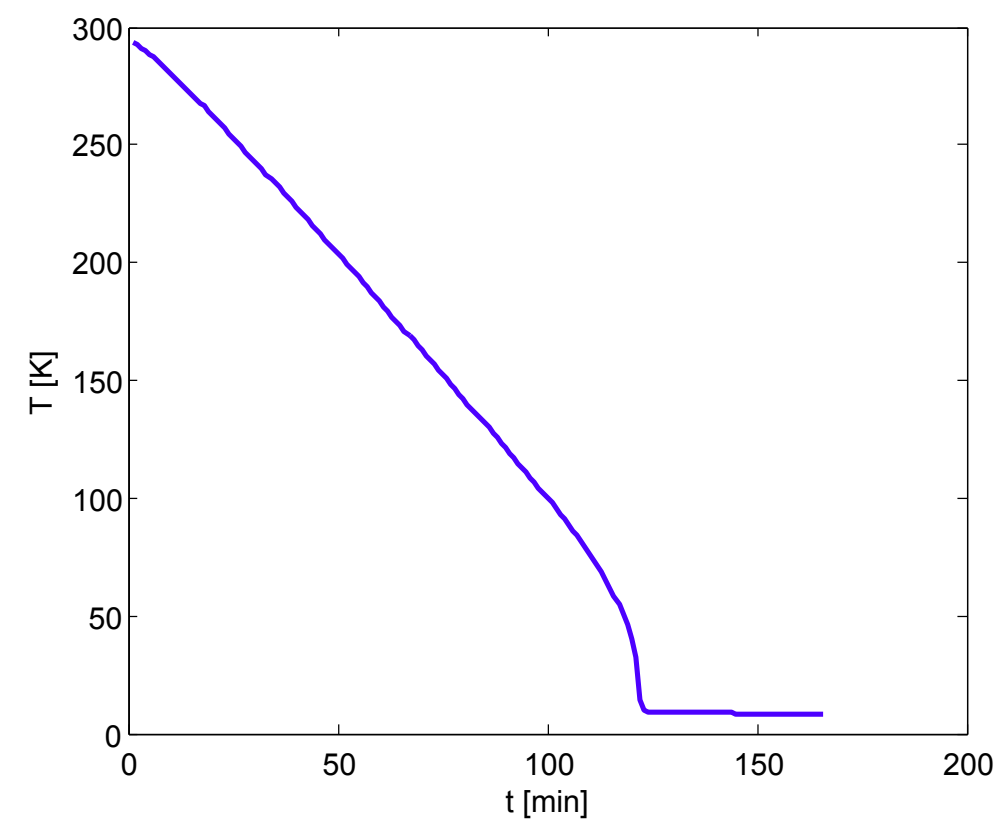

Figure 4.4: Cooling curve for the new nozzle assembly. Temperatures are as read from the silicon diode, which has been found to be accurate to within less than $2 \mathrm{~K}$.

To characterise the performance of the new source in the HeSE spectrometer, several beam profile measurements have been performed. A beam profile is a spin-echo measurement where the intensity of the specularly reflected beam is recorded as a function of the current in the first solenoid only (while the second solenoid is turned off). The polarisation of the beam is changed periodically, giving an oscillation of the signal measured in the detector.

The lowest nozzle temperature that can be maintained while the helium flow rate is sufficient to give a beam useful for HeSE measurements (i.e. with a beam intensity above the noise level) is a nominal temperature of $10.0 \mathrm{~K}$. Figure 4.5 shows a polarisation curve recorded at specular reflection from a clean $\mathrm{Cu}(111)$ crystal surface at this minimum

\footnotetext{
${ }^{1}$ The system is unbaked, hence there is a residual water vapour pressure in the chamber.
} 
nominal nozzle temperature. The apparent oscillations are a result of the mean beam energy while the envelope function is defined by the energy spread in the beam. By Fourier transforming the data, a direct energy spectrum can be obtained, as shown in the bottom panel of Figure 4.5. The peak indicates a beam energy of $2.5 \mathrm{meV}$ with a FWHM of $0.3 \mathrm{meV}$. Equation 4.1 relates this beam energy to a temperature of $11.8 \mathrm{~K}$, which is less than $2 \mathrm{~K}$ off the nominal temperature of $10.0 \mathrm{~K}$.

Beam profiles have been performed for a range of different nominal nozzle temperatures to calibrate them against the actual beam energy, confirming that the silicon diode readings are accurate to within $2 \mathrm{~K}$ over the entire temperature range between room temperature and the minimum. A number of low temperature calibration points are summarised in Table 4.1. The nominal minimum temperature of $8.0 \mathrm{~K}$ reached in Figure 4.4 thus corresponds to an actual temperature of approximately $10 \mathrm{~K}$ - essentially the temperature which the coldhead is rated to.

\begin{tabular}{l|lllll}
\hline nominal temperature $[\mathbf{K}]$ & 10.0 & 15.0 & 25.0 & 37.0 & 50.0 \\
\hline actual temperature $[\mathbf{K}]$ & 11.8 & 16.6 & 26.2 & 38.1 & 51.1 \\
\hline
\end{tabular}

Table 4.1: Calibration of the nominal nozzle temperatures of the new source and the actual temperature measured in the beam with a spin-echo measurement.

These experiments demonstrate that the silicon diode, which is installed on the outside of one of the copper clamping tubes, is sufficiently close to the nozzle to provide fairly reliable readings. Furthermore, the minimum operating temperature of $11.8 \mathrm{~K}$ is very close to the $10 \mathrm{~K}$ that the Sumitomo coldhead is rated to, illustrating that the new heat shielding design indeed works extremely well. The new source can thus operate at nozzle temperatures between 11.8 and $300 \mathrm{~K} .{ }^{2}$ As mentioned in Section 4.1, the energy resolution of the HeSE spectrometer goes with the power $E_{0}^{3 / 2}$ or equivalently $T_{\text {nozzle }}^{3 / 2}$ A nozzle temperature $T_{\text {nozzle }}=11.8 \mathrm{~K}$ or beam energy $E_{0}=2.5 \mathrm{meV}$ therefore corresponds to an improvement in resolution of a factor of $(37 / 11.8)^{3 / 2}=5.5$. Since a nominal beam energy of $8 \mathrm{meV}$ provided an energy resolution of $3 \mu \mathrm{eV}$, the new source improves the instrument's resolution to $550 \mathrm{neV}$. The new source thus extends the upper limit of the measurement range accessible with the Cambridge HeSE spectrometer from 680 ps to $3.8 \mathrm{~ns}$, making the investigation of a whole new range of slowly diffusing adsorbates possible.

\footnotetext{
${ }^{2}$ The upper limit is currently determined by the temperature rating of the silicon diode.
} 

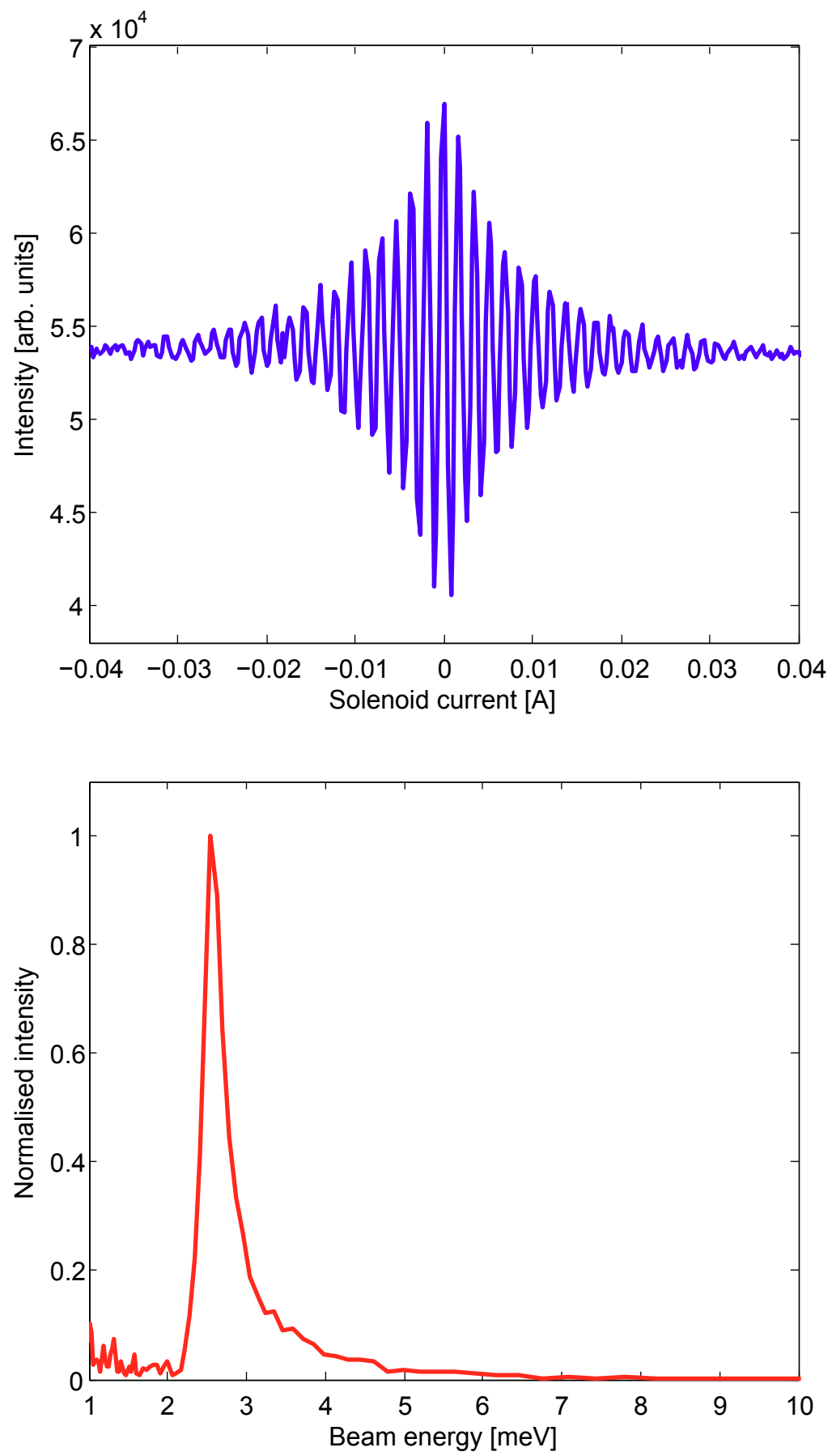

Figure 4.5: Spin-echo measurement at a nominal nozzle temperature of $10.0 \mathrm{~K}$. The top panel shows a periodic variation of the polarisation as a function of ingoing solenoid current, where the oscillations are a result of the mean beam energy and the envelope function is defined by the energy spread in the beam. Fourier transforming the measurement gives the energy spectrum of the beam, as shown in the bottom panel, indicating a beam energy of $2.5 \mathrm{meV}$, or actual nozzle temperature of $11.8 \mathrm{~K}$, and a FWHM of $0.3 \mathrm{meV}$. 
At a beam energy of $8.0 \mathrm{meV}$, HeSE measurements can be performed up to a momentum transfer of approximately $4.0 \AA^{-1}$ in favourable conditions [23] (cf. for example Figure 5.18), corresponding to a $70^{\circ}$ angle of incidence on the crystal. Since the kinetic energy of the incident helium atom is given by

$$
E_{i}=\frac{\hbar^{2} k_{i}^{2}}{2 m}
$$

where $m$ is its mass, $\hbar$ the reduced Planck constant, and $k_{i}$ the incident wave vector, the momentum transfer range goes with the square root of the beam energy. At an energy of $2.5 \mathrm{meV}$, the measurement range is thus reduced to $\frac{4.0 \AA^{-1}}{\sqrt{8.0 \mathrm{meV} / 2.5 \mathrm{meV}}}=2.2 \AA^{-1}$. Ideally, it is desirable to measure adsorbate dynamics up to a momentum transfer value beyond the diffraction point of the substrate to map out jump diffusion on the length scale of the substrate. Typical lattice constants of metal surfaces are on the order of $\sim 2 \AA$, which corresponds to approximately $3 \AA^{-1}$ in momentum transfer space (for example, $\mathrm{Cu}(111)$ has a diffraction position at $2.84 \AA^{-1}$ along the $<11 \overline{2}>$ azimuth [40]). However, while measurements over a relatively large momentum transfer range are required to differentiate between Brownian motion and jump diffusion - the beginning of a sinusoidal curve and a quadratic cannot easily be distinguished (cf. Figure 2.7) - it is not strictly necessary to measure up to the diffraction point, and a jump diffusion mechanism can be identified as long as a peak in the curve is resolved. With the new beam source, it is therefore possible to perform diffusion studies of slow-moving adsorbate species on timescales up to 3.8 ns on the Cambridge HeSE spectrometer.

First experiments putting the performance of the new source to the test have been carried out by Holly Hedgeland [69], investigating the diffusion of benzene on a $\mathrm{Cu}(100)$ surface. Prior measurements using the original source at a beam energy of $10 \mathrm{meV}$ showed intermediate scattering functions (ISFs) that proved difficult to interpret as the resolution was insufficient to show clear curvature in the curves [28]. To describe the data, assumptions about the static level had to be made, making interpretation difficult.

After the installation of the new source, additional measurements were performed at a beam energy of $3.6 \mathrm{meV}$, extending the spin-echo time range to $2.2 \mathrm{~ns}$ [69]. Two typical curves are presented in Figure 4.6. The blue dots are data at $0.29 \AA^{-1}$, while the red dots show measurements at $0.50 \AA^{-1}$. The green dashed line indicates the maximum spin-echo time that can be achieved with the nominal beam energy of the HeSE spectrometer, $8.0 \mathrm{meV}$, while the grey dashed line illustrates the maximum accessible spin-echo time in the previous benzene $/ \mathrm{Cu}(100)$ measurements [28]. Due to the noise in the data, it is 
extremely difficult to analyse the curvature in the points left of either dashed line. With the improved resolution from the lower beam energy, however, the decay is resolved up to $2.2 \mathrm{~ns}$ and the shape of the curves can be distinguished very well, as illustrated by the black and grey solid lines. These experiments demonstrate the clear benefit of increased machine resolution.

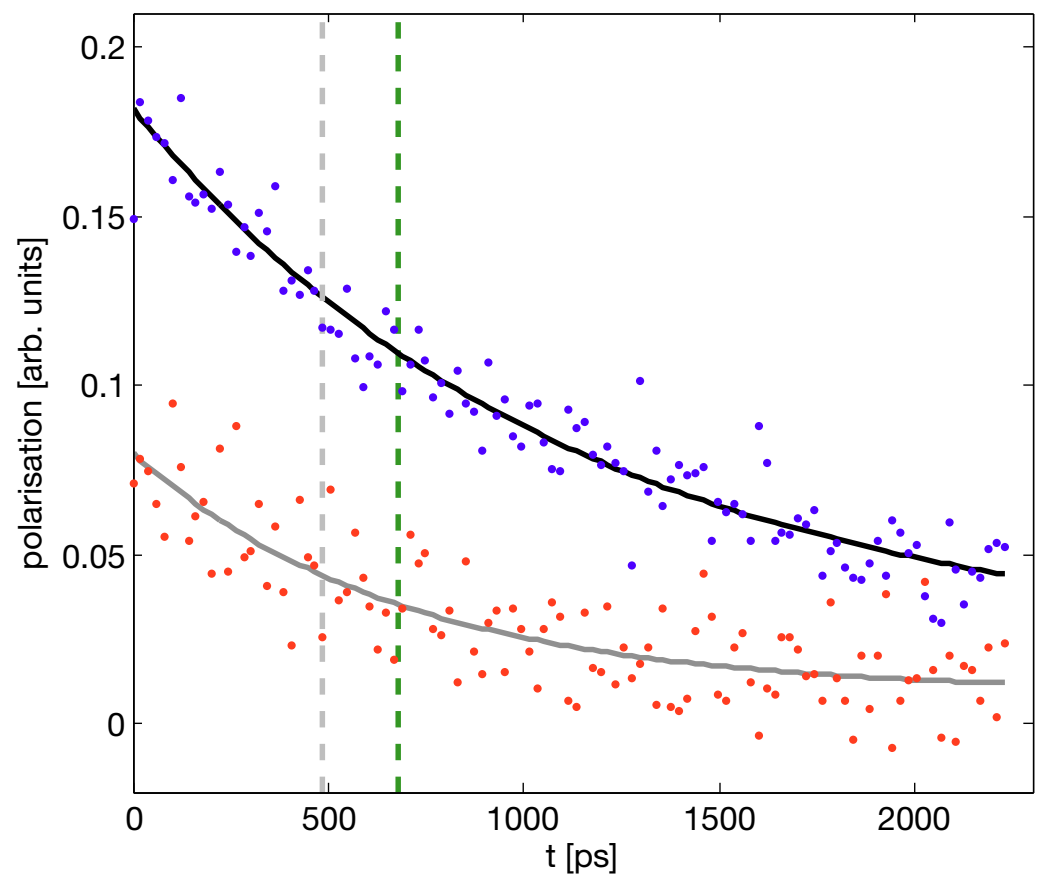

Figure 4.6: Polarisation curve of benzene $/ \mathrm{Cu}(100)$ measured by Holly Hedgeland using the new source at a beam energy of $3.6 \mathrm{meV}$. Data at $\Delta \boldsymbol{K}=0.29 \AA^{-1}$ and $0.50 \AA^{-1}$ are shown as blue and red dots, respectively. The black and grey solid lines represent an exponential curve fitted through the data. The green dashed line illustrates the maximum spin-echo time accessible with an $8 \mathrm{meV}$ beam, $680 \mathrm{ps,}$ which in the case of benzene/ $\mathrm{Cu}(100)$ does not provide a sufficient range to accurately determine the curvature of the data. A second dashed line in light grey marks the maximum spin-echo time accessible in the previous benzene $/ \mathrm{Cu}(100)$ measurements of $490 \mathrm{ps}$, using a beam energy of $10 \mathrm{meV}$.

\subsection{Effects of size and shape of the skimmer and its mount}

As mentioned in Section 4.1, attenuation of the low temperature beam has been observed in the original HeSE source, which was suggested to be a result of backscattering of the 
beam in the nozzle-skimmer region [24]. The skimmer mount for the new source has therefore been designed to move the skimmer away from the chamber wall to minimise the effect of backscattering from the wall.

\subsubsection{Design}

Figure 4.7 shows an Autodesk Inventor three-quarter cross section drawing of the new skimmer mount and a typical skimmer. All components of the skimmer mount are made from stainless steel and only shown in different colours for illustration purposes. The plate shown in red is installed in the source chamber and defines the position of the skimmer in the chamber. This plate acts as a point of reference, defining the alignment of the skimmer with respect to all other apertures along the ingoing beam arm of the instrument. It is identical to that of the original skimmer mount and so did not need to be exchanged when the new source was installed on the HeSE spectrometer, thus ensuring the alignment of the skimmer was maintained. The second plate, illustrated in green, has a recess with a tight fit onto the first plate to ensure positioning of the skimmer mount in the precise same location each time it is exchanged. The tube part (blue) is designed to have a sloppy fit onto the part shown in green so that it can be positioned precisely in the centre of the second mounting ring by grub screws pushing it sideways. This mechanism permits positioning of the skimmer on the exact centreline of the beam axis of the spectrometer. The skimmer itself, shown in cyan, is clamped between the flat top of the tube component, in blue, and a screw cap, which is shown in orange.

The two main differences between this new skimmer mount design and the original one are the added length from the tube component (blue) and the use of a flat screw cap to clamp the skimmer brim instead of a ring bolted down by several screws. The added length removes the foot of the skimmer mount from the direct line of sight of the helium gas expanded through the nozzle and moves the skimmer further away from the chamber wall. The clamping mechanism by screw thread has the advantage that no bolt heads protrude into the area next to the skimmer but instead the skimmer mount has a completely flat end. This design is intended to minimise the beam attenuation which was found to be dependent on the head size of the bolts positioned next to the skimmer in the original design [70] and allows for an overall slimmer skimmer mount. The new skimmer mount installed in the source chamber of the HeSE spectrometer is shown in the photograph in Figure 4.3 (B). The dimensions of the new nozzle assembly are such that 


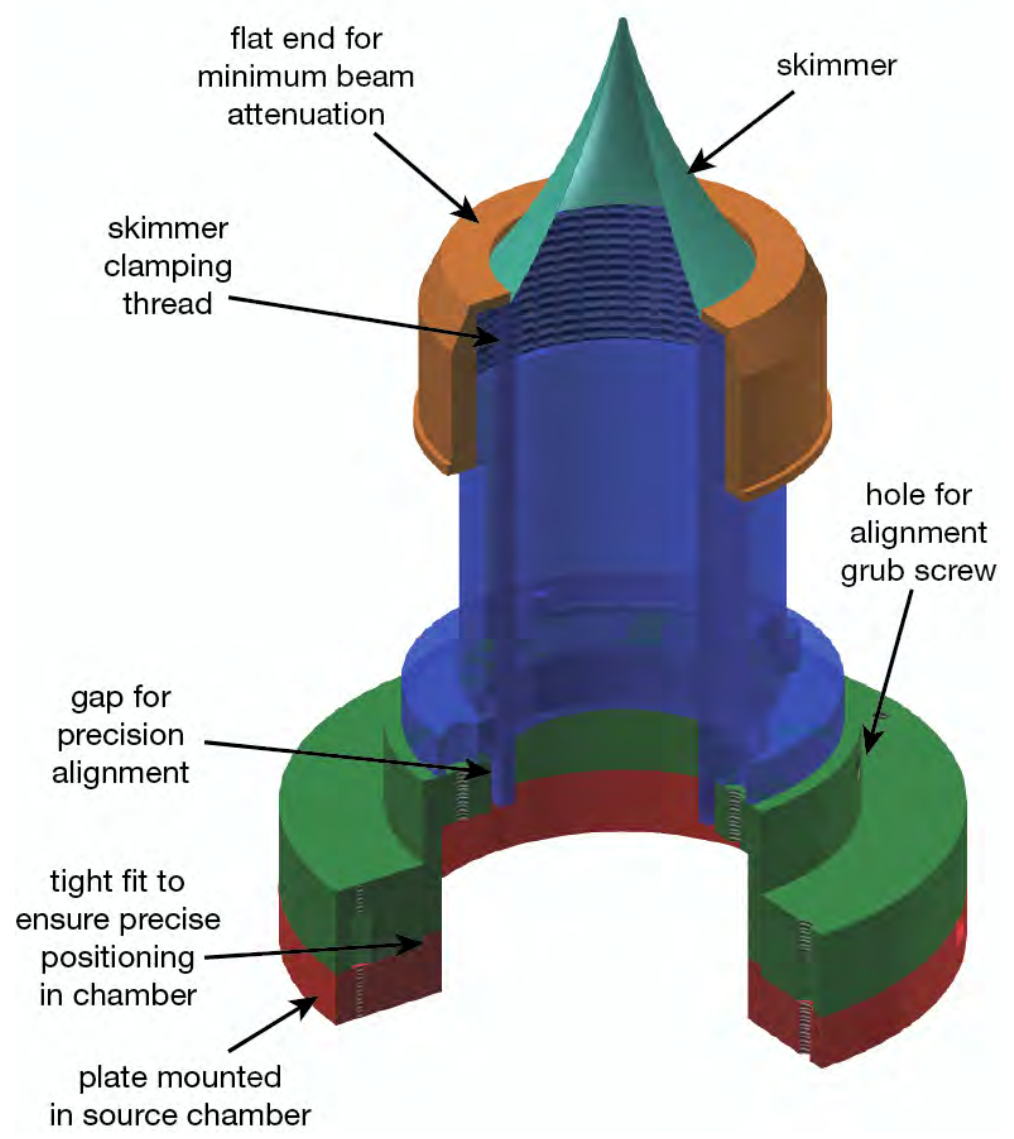

Figure 4.7: Autodesk Inventor drawing showing a three-quarter cross section view of the new skimmer mount and a typical skimmer. The plate shown in red is installed in the source chamber and defines the alignment of the skimmer mount with respect to the ingoing beam arm of the spectrometer. The skimmer is clamped tightly between a screw cap (orange) and the tube component (blue), which can be positioned in the centre of the component shown in green by four grub screws pushing it sideways. A tight fit between the two plates (red and green) ensures reproducible positioning of the skimmer in the source chamber.

a standard model 2 Beam Dynamics skimmer [54] in the new skimmer mount cannot be damaged accidentally, as the maximum travel on the source manipulator positions the nozzle at approximately $2 \mathrm{~mm}$ from the tip of the skimmer.

When the new skimmer mount was installed on the HeSE spectrometer, alignment of the skimmer along the beam axis defined by the apertures in the ingoing beam arm was checked visually using a theodolite and by comparing the pressure rise in the scattering chamber created by the beam for a certain nozzle temperature and pressure. 
Both methods confirmed, for the first time, that the alignment method using a tight fit between the two mounting plates works reliably.

\subsubsection{Performance}

In the original HeSE source, a $0.5 \mathrm{~mm}$ diameter model 2 Beam Dynamics skimmer [54] was used. Initial tests of the new source were performed with an identical skimmer to allow direct comparison.

The best measure for the beam intensity is the pressure rise in the scattering chamber, created by the beam. Figure 4.8 shows measurements of the pressure rise as a function of nozzle flow for a low nozzle temperature and a room temperature nozzle. The flow through the nozzle, $\hat{T}$, has been related to the source conditions empirically [60] by

$$
\hat{T}=C\left(\frac{T_{c}}{T_{0}}\right)^{1 / 2} p_{0} d^{2}
$$

where $p_{0}$ is the nozzle pressure, $T_{0}$ the nozzle temperature, $T_{c}$ the temperature of the vacuum chamber, $d$ the nozzle diameter and $C$ is an empirical flow constant for the particular gas used. At a given nozzle temperature, the nozzle flow is proportional to the nozzle pressure. For measurements at different nozzle temperatures, however, the flow through the nozzle is a more comparable unit than the nozzle pressure.

Figure 4.8 compares the beam intensity of the original HeSE source (blue circles) with that of the new source with identical skimmer (red triangles). The signal intensity is significantly increased both at low and at room temperature. Since the low temperature data are for a nozzle temperature of $32 \mathrm{~K}$ for the original and $37 \mathrm{~K}$ for the new source, additional data at $25 \mathrm{~K}$ is shown (grey), confirming that the increase in pressure is not simply due to the slightly higher nozzle temperature.

Interestingly, the maximum in the curve is moved to a smaller nozzle flow in the new source. The increased overall signal level seems to imply that the new skimmer mount design has indeed reduced the level of attenuation in the beam, while the earlier peak suggests that a new source of attenuation has been created instead. For HeSE experiments, the nozzle pressure is usually set to the peak value to maximise the signal intensity. 

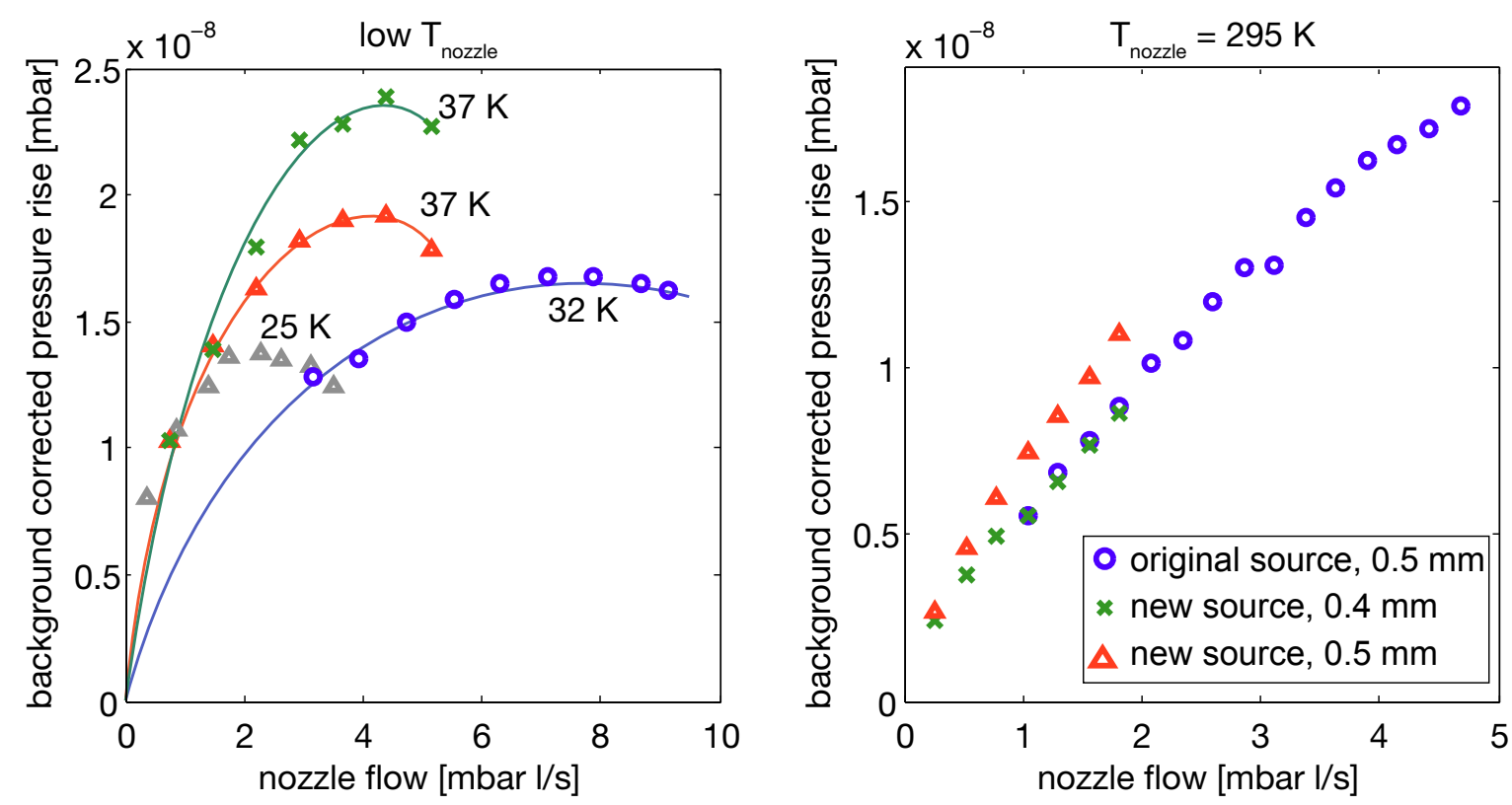

Figure 4.8: Measurements of the pressure rise from the helium beam in the scattering chamber, comparing the performance of the new source with that of the original one. All pressures are corrected for the gauge factor of helium and the background in the chamber. Data for the original source are shown in blue circles (measured by Pepijn Kole [25]), for the new source with the standard $0.5 \mathrm{~mm}$ skimmer in red triangles and for the new source with a $0.4 \mathrm{~mm}$ diameter skimmer in green crosses. The panel on the left is for low nozzle temperatures while the data in the right-hand side panel were recorded using a room temperature nozzle. The comparison of the performance at a low nozzle temperature is between a curve at $32 \mathrm{~K}$ for the original and $37 \mathrm{~K}$ for the new source. To confirm that the signal intensity is indeed increased in the new source, data for the new source at $T_{\text {nozzle }}=25 \mathrm{~K}$ are included as grey triangles (for a $0.5 \mathrm{~mm}$ skimmer).

To investigate the effect of the chamber wall experimentally, the low nozzle temperature measurements have been repeated with an aluminium plate installed on the skimmer mount, as illustrated in Figure 4.9. Care has been taken to position the bolts holding the artificial "wall" so that their heads do not protrude beyond the top of the skimmer mount to remove the effect of the bolt heads described above [70]. Figure 4.10 compares the pressure rise of the new source in the original setup and with the "wall" moved closer to the skimmer. No change in the beam intensity is observed, neither with a straight nor a bent plate. The increase in intensity from the new source compared to the original one thus seems to result purely from the flat, slim geometry of the skimmer mount in the immediate vicinity of the skimmer. Simulations describing the principal sources of backscattering in the source had suggested that moving the chamber wall position relative to the skimmer changes the beam intensity [24]. These results seem to be contradicted 
by the experiments suggesting that the skimmer-to-wall distance does not influence the beam intensity significantly. A possible explanation for this discrepancy is discussed in Section 4.3.3.
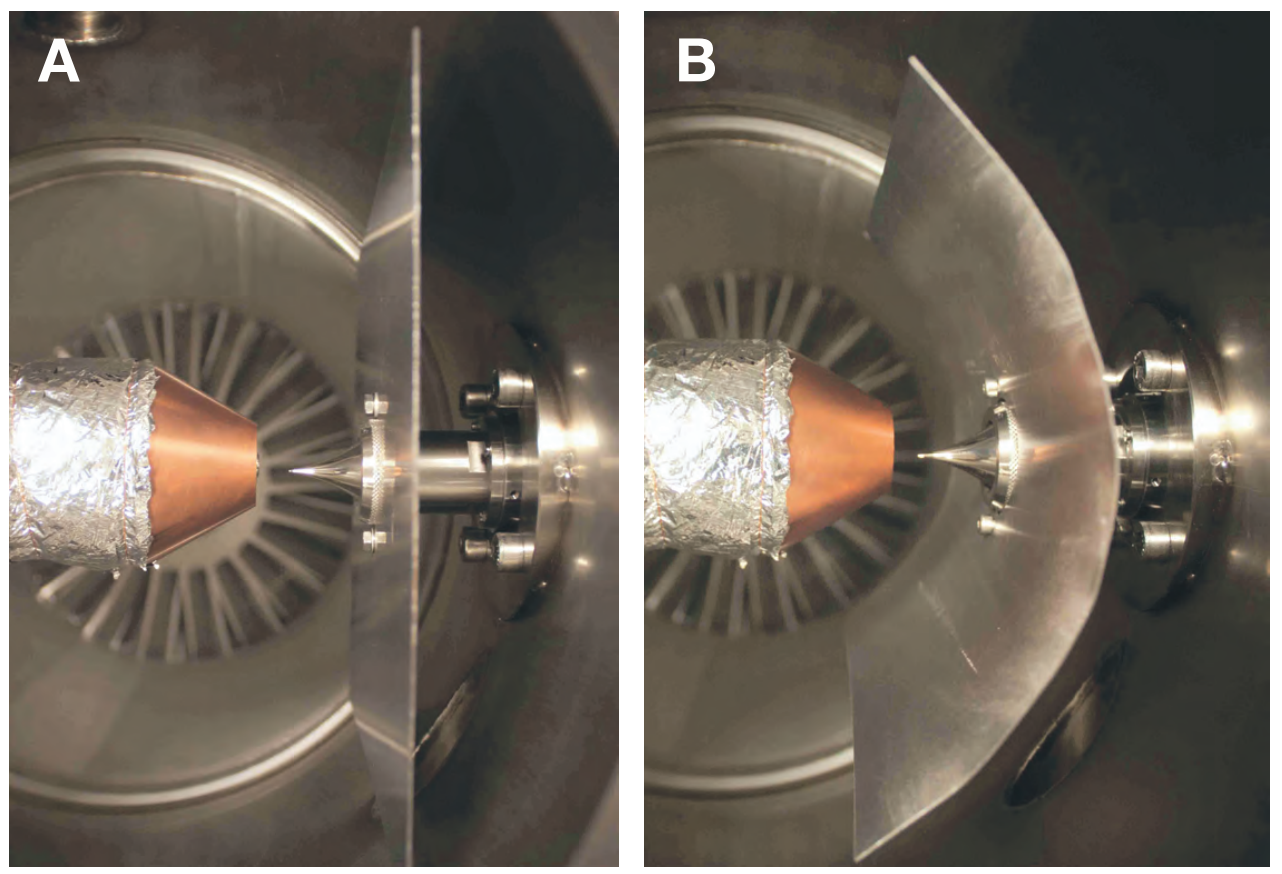

Figure 4.9: Photographs illustrating the experiments of beam intensity as a function of skimmer-to-wall distance. (A) shows a straight "wall" installed on the front of the skimmer mount, while the plate in (B) has a curvature similar to that of the chamber wall, thus allowing a direct comparison of the effect of the distance between skimmer and chamber wall.

Another possible explanation for the early onset of attenuation in the new source is an increased pressure in the first differential pumping stage, behind the skimmer. If the expansion process continues beyond the skimmer, it is conceivable that helium collides with the wall of the long, slim tube in the new design, attenuating the beam after skimming. To study the influence of the pressure behind the skimmer experimentally, a skimmer of identical shape (model 2 Beam Dynamics) but with a smaller diameter of $0.39 \mathrm{~mm}$ has been installed, which should reduce the helium pressure inside the skimmer mount and in the first pumping stage. The pressure rise in the scattering chamber for the new source with the $0.39 \mathrm{~mm}$ skimmer is shown in green in Figure 4.8. For a cold beam, the desired increase in signal is indeed observed, yet the turnover point has barely moved. At room temperature, on the other hand, the beam intensity is somewhat reduced for a smaller skimmer. 


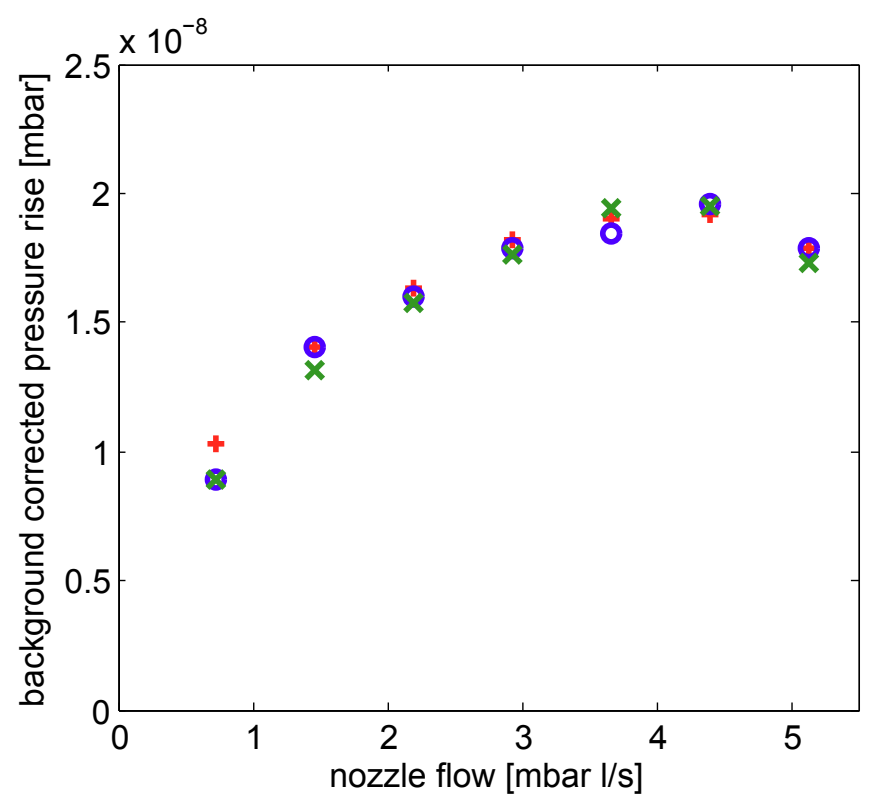

Figure 4.10: Measurements of the pressure rise from the helium beam in the scattering chamber, comparing the standard setup of the new source with a wall moved closer to the skimmer, as illustrated in Figure 4.9. Data for the standard setup of the new source are shown in red, while blue circles and green crosses are for the setups shown in Figure 4.9 (A) and (B), respectively. No significant difference between the three configurations is apparent. All data have been recorded at a nozzle temperature of $37 \mathrm{~K}$ and all pressure rise values are corrected for the gauge factor of helium and the chamber background pressure.

\subsubsection{Discussion and future work}

The results from experiments studying the effect of skimmer-to-wall distance and skimmer diameter suggest that there are two competing interference processes occuring inside and outside the skimmer. At room temperature, the attenuation in front of the skimmer is the dominating effect, resulting in a small decrease in signal level for a smaller diameter skimmer which has a slightly longer tip. At a low nozzle temperature, on the other hand, the attenuation within the skimmer mount appears to be the dominating factor, explaining the increase in signal for a smaller skimmer.

In Section 4.1, two different types of sources were reviewed: the Fenn type source operating at low source chamber pressures, and the Campargue type where a higher pressure in the chamber creates a zone of silence and the skimmed beam continues to expand in the first pumping stage [64]. The effect of low temperature beam attenuation in the original source of the HeSE spectrometer was first observed when high capacity 
diffusion pumps were used, and the simulations suggesting backscattering from the skimmer mount and wall as principal attenuation sources were performed for this setup [24]. The source was later upgraded to a dry system, using turbomolecular pumps and a scroll backing pump to avoid contamination of the helium-3 recycling system with pump oil [25]. The change to a pump of lower capacity may have resulted in a transition from the Fenn regime towards the Campargue regime, which would explain the discrepancies between simulations and experiments with the new source.

A good indication of the regime the source operates in, is the nozzle-skimmer distance which gives maximum intensity in the beam. Figure 4.11 shows the pressure rise in the scattering chamber as a function of nozzle-skimmer distance for a nozzle temperature of $37 \mathrm{~K}$ and a nozzle pressure of 20 bar. A flat maximum in the curve occurs between $9 \mathrm{~mm}$ and $14 \mathrm{~mm}$, which is on the lower end of the Fenn type source [64]. The data therefore suggest that the HeSE source operates closer to the Campargue regime than originally suspected, making continued expansion beyond the skimmer likely.

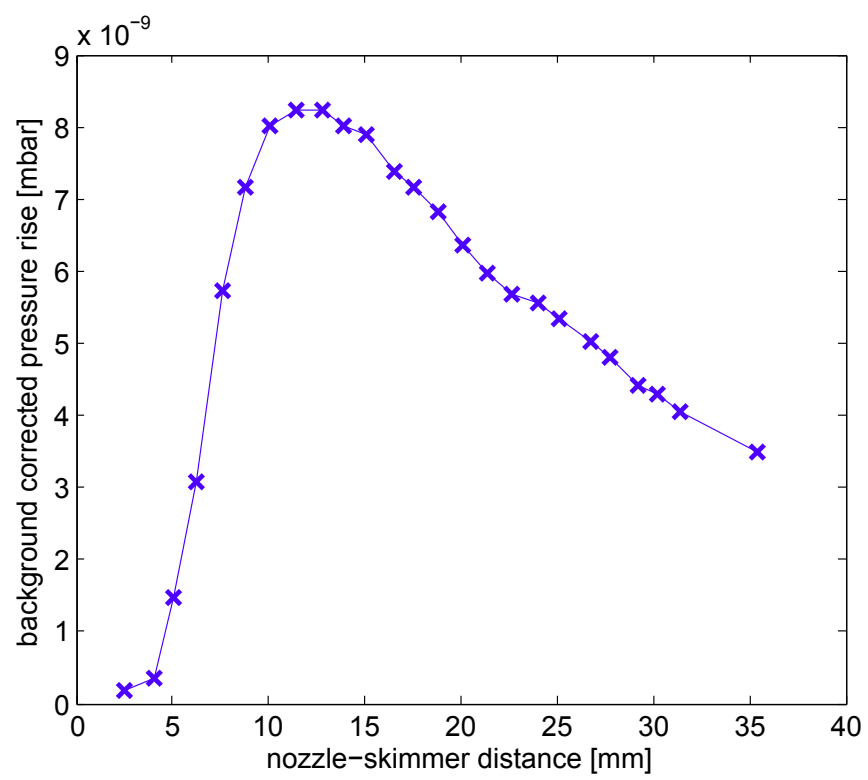

Figure 4.11: Helium beam pressure rise in the scattering chamber as a function of nozzleskimmer distance. The data shown are for a nozzle temperature of $37 \mathrm{~K}$ and a nozzle pressure of 20 bar. A flat maximum is observed between 9 and $14 \mathrm{~mm}$.

Campargue reported that altering the skimmer angle had a great effect on the beam intensity in a Campargue type source, while little influence was observed in the Fenn regime [64]. While the precise values of optimum skimmer angle cannot simply be taken from Campargue's publication as the experiments were performed using a 
room temperature nozzle, the behaviour should be qualitatively similar at low nozzle temperatures. Therefore, further experiments on the HeSE source could be performed to investigate the effect of the skimmer angle. The model 2 Beam Dynamics skimmers used in the experiments above have a $25^{\circ}$ interior included angle, which is fairly small. Investigating the performance of the source with a wider angle skimmer, such as for example a model 19.6 Beam Dynamics skimmer with an interior included angle of $55^{\circ}$ [54], should allow a final distinction of the skimming regime of the source and thus enable a more precise optimisation of the skimmer geometry. In addition, a continued investigation of the skimmer size, reducing the hole diameter below $0.39 \mathrm{~mm}$, could increase the beam intensity further.

\subsection{Conclusions}

An improved high intensity supersonic helium beam source has been presented in this chapter. A new, straight nozzle assembly with a heat shielding tube of polished copper reaches beam energies as low as $2.5 \mathrm{meV}$, improving the energy resolution of the HeSE spectrometer by a factor of 5.5. First experiments measuring the slow diffusion of benzene/ $\mathrm{Cu}(100)$ have demonstrated the performance of the new source [69]. With the installation of the new HeSE detector by David Chisnall, giving a vast increase in signal intensity [30], measurements at such low beam energies become even more attractive, as the new detector reduces the issue of intensity loss due to the overfocussing of the beam.

A new skimmer mount design creates an increase in the signal intensity by approximately $20 \%$. The improved performance can largely be attributed to the flat, slim design in the immediate vicinity of the skimmer. The effect of the distance between skimmer base and chamber wall has been investigated, suggesting that backscattering from the wall does not attenuate the beam significantly. Reducing the skimmer diameter resulted in an increase in intensity at low nozzle temperatures. 


\section{Chapter 5}

\section{The dynamics of cyclopentadienyl on $\mathrm{Cu}(111)$}

This chapter presents helium-3 spin-echo (HeSE) measurements of the dynamics of cyclopentadienyl $(\mathrm{Cp}), \mathrm{c}-\mathrm{C}_{5} \mathrm{H}_{5}$, on $\mathrm{Cu}(111)$. $\mathrm{Cp}$ is a widely used ligand in organometallic chemistry, forming sandwich complexes with metal centres such as in ferrocene, $\mathrm{Fe}(\mathrm{Cp})_{2}$, and other metallocenes, $\mathrm{M}(\mathrm{Cp})_{2}$. In these complexes $\mathrm{Cp}$ is negatively charged with the electron delocalised in the aromatic ring and all $\mathrm{C}-\mathrm{C}$ bond lengths essentially equal [71] and can be used to control the oxidation state of isolated metal ions. When adsorbed on a metal surface, $\mathrm{Cp}$ is anticipated to be a prototypical anionic organic adsorbate, exhibiting strong lateral interactions and ionic bonding to the substrate [72].

The net charge and dipole moment of an organic adsorbate are determining factors in the alignment between electronic states of the adsorbed molecule and the substrate [73,74]. Electrostatic rearrangements at the interface also control long-range forces and hence self-organisation during the early stages of thin-film growth [75]. Strongly bound ionic species, together with appropriate functionalisation, offer a range of new possibilities for self-assembly but little is known of their behaviour. Therefore, the dynamical behaviour of $\mathrm{Cp} / \mathrm{Cu}(111)$ is studied to gain a quantitative understanding of anionically adsorbed systems. The fivefold geometry of Cp suggests that these molecules do not have a preferred orientation when adsorbed on the hexagonal $\mathrm{Cu}(111)$ surface, thus making it an ideal candidate for studying rotational as well as translational motion.

In the following section, the literature background on $\mathrm{Cp}$ adsorption on metal surfaces is summarised. Subsequently, the experimental measurements of the dynamics of $\mathrm{Cp} / \mathrm{Cu}(111)$ are presented and a detailed analysis is performed by molecular dynamics 
simulations, providing a value for the friction coefficient. A global analysis of all data combined (rather than investigating each spectrum individually), using Bayesian statistics, gives a precise measure of the energy difference between the two preferred adsorption sites. Finally, a first experimental investigation of the intracell diffusion of an adsorbate providing a value for friction and spring constant are presented. The experimental study is supplemented by first-principles density functional theory calculations carried out by Sacchi and Jenkins from the Department of Chemistry at Cambridge.

\subsection{Literature background}

This section provides background information on the structure of $\mathrm{Cp}$ and summarises prior studies of the adsorption behaviour of $\mathrm{Cp}$ on metal surfaces, in particular copper.

\subsubsection{The structure of Cp}

Cyclopentadienyl is not chemically stable in the gas form but only exists in metal complexes or adsorbed on a surface. Its dimensions have been determined as part of structure determinations of various metallocenes [76].

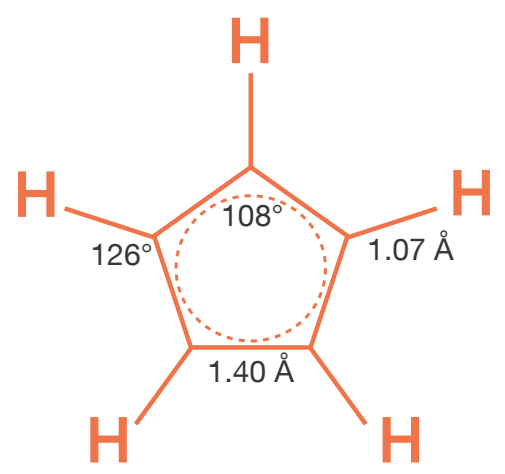

Figure 5.1: Schematic illustration of the structure of Cp. Values for bond lengths and angles are taken from [71] and [77].

The crystal structure of ferrocene was first measured by Dunitz et al. in X-ray diffraction studies $[71,78]$. The authors reported planar $\mathrm{Cp}$ rings where all $\mathrm{C}-\mathrm{C}$ bonds are of essentially the same length, with an average $\mathrm{C}-\mathrm{C}$ bond length of $1.40 \pm 0.02 \AA$. They estimated the $\mathrm{C}-\mathrm{H}$ bond lengths as $1.05 \AA$, yet could not measure them experimentally. Applegate et al. later determined the $\mathrm{C}-\mathrm{H}$ bond lengths of $\mathrm{Cp}$ in ferrocene by first 
principles calculations and found an average of $1.07 \AA[77]$. While the distance between $\mathrm{Cp}$ rings and metal centre varies for different metallocenes, the structure of the Cp rings remains essentially the same [76]. All $\mathrm{C}-\mathrm{C}-\mathrm{C}$ angles are $108^{\circ}$ and $\mathrm{H}-\mathrm{C}-\mathrm{C}$ angles $126^{\circ}$, as expected for a regular pentagonal structure. Figure 5.1 summarises the overall geometry of Cp.

\subsubsection{Cp adsorption on copper surfaces}

Little is known about the adsorption of $\mathrm{Cp}$ on copper surfaces, in particular no prior studies of $\mathrm{Cp} / \mathrm{Cu}(111)$ exist. However, $\mathrm{Cp} / \mathrm{Cu}(100)$ was studied using thermal desorption spectroscopy (TDS) and Auger electron spectroscopy (AES) [79]. Sun et al. demonstrated that cyclopentadiene, $\mathrm{c}^{-} \mathrm{C}_{5} \mathrm{H}_{6}(\mathrm{CpH})$, adsorbs dissociatively on $\mathrm{Cu}(100)$ at low exposures, producing $\mathrm{Cp}$ as the stable adsorbate species at temperatures below $600 \mathrm{~K}$. Cyclopentadiene was adsorbed at $100 \mathrm{~K}$ and the sample subsequently heated with a heating rate of $3 \mathrm{~K} / \mathrm{s}$ while the desorbing gas was monitored. The results suggested that dissociative adsorption first produced $\mathrm{Cp}$ and atomic hydrogen, the latter of which subsequently recombined at temperatures between $335 \mathrm{~K}$ and $360 \mathrm{~K}$ to desorb as molecular hydrogen, $\mathrm{H}_{2}$. Deuterium adsorption prior to and after the $\mathrm{c}_{-} \mathrm{C}_{5} \mathrm{H}_{6}$ adsorption was used to confirm the existence of the reaction intermediate, Cp. TDS and AES measurements showed that pre-adsorbed deuterium atoms suppress the dehydrogenation of $\mathrm{c}_{-} \mathrm{C}_{5} \mathrm{H}_{6}$ which the authors interpreted as the occupation of active sites by deuterium atoms. On the other hand, dosing deuterium atoms after $\mathrm{c}_{-} \mathrm{C}_{5} \mathrm{H}_{6}$ adsorption resulted in the formation of $\mathrm{C}_{5} \mathrm{H}_{5} \mathrm{D}$, evidence for hydrogenation of $\mathrm{Cp}$ before desorption.

\subsubsection{Cp adsorption on other metal surfaces}

A range of experimental and theoretical work on the adsorption behaviour of $\mathrm{Cp} / \mathrm{Pt}(111)$ can be found in the literature. In several EELS, TDS and work function studies, Avery investigated the adsorption of cyclopentane, cyclopentene and cyclopentadiene and the dehydrogenation of these species to a stable reaction intermediate, $\mathrm{Cp}$, on $\mathrm{Pt}(111)$ [80-83].

Cyclopentene, $\mathrm{C}_{5} \mathrm{H}_{8}\left(\mathrm{CpH}_{3}\right)$, on $\mathrm{Pt}(111)$ was found to result in molecular adsorption at temperatures below $250 \mathrm{~K}[80,81]$. In this phase, the molecules are $\mathrm{di}-\sigma$ bonded in a tilted geometry, undergoing a rehybridisation from $\mathrm{sp}^{2}$ to $\mathrm{sp}^{3}$. In TDS measurements, multilayer desorption of cyclopentene was observed at $145 \mathrm{~K}$, while the monolayer partially desorbs 
at $285 \mathrm{~K}$. In addition, dehydrogenation to Cp occurs between $250 \mathrm{~K}$ and $300 \mathrm{~K}$, resulting in desorption of $\mathrm{H}_{2}$ at $305 \mathrm{~K}$ and adsorbed $\mathrm{Cp}$ molecules. Avery emphasised the similarity of $\mathrm{Cp}$ adsorbates on transition metal surfaces and in metallocene complexes, suggesting $\pi$-bonding between the adsorbate molecules and the substrate metal atoms, resulting in an adsorption geometry with the molecular ring parallel to the surface. Work function measurements showed a decrease in the work function of $1.55 \mathrm{eV}$ upon adsorption of a full layer of cyclopentene and of $1.60 \mathrm{eV}$ after dehydrogenation to Cp. Upon further heating to beyond $480 \mathrm{~K}, \mathrm{Cp}$ decomposes to form surface carbon and desorbing $\mathrm{H}_{2}$.

Cyclopentane, $\mathrm{C}_{5} \mathrm{H}_{10}\left(\mathrm{CpH}_{5}\right)$, on the same substrate showed very similar behaviour [82]. Avery reported molecular adsorption with the ring approximately parallel to the surface at $90 \mathrm{~K}$, followed upon heating by partial molecular desorption with a peak at $200 \mathrm{~K}$, and partial dehydrogenation to cyclopentene around $180 \mathrm{~K}$ to $200 \mathrm{~K}$. Similarly to the behaviour after direct adsorption of cyclopentene, thermal treatment to above $250 \mathrm{~K}$ results in dehydrogenation to $\mathrm{Cp}$. The work function change upon adsorption of cyclopentane was reported as $0.82 \mathrm{eV}$.

Finally, Avery also examined the adsorption behaviour of cyclopentadiene on $\operatorname{Pt}(111)$ and reported a disproportionation reaction upon adsorption at $90 \mathrm{~K}$ [83] where simultaneous dehydrogenation and hydrogenation resulted in coadsorbed $\mathrm{Cp}$ and cyclopentene species. Treatment to temperatures between $280 \mathrm{~K}$ and $350 \mathrm{~K}$ initiated dehydrogenation of cyclopentene to $\mathrm{Cp}$ and desorption of excess hydrogen as $\mathrm{H}_{2}$, indicating that $\mathrm{Cp}$ is the most thermally stable $\mathrm{C}_{5}$ species on a $\mathrm{Pt}(111)$ surface.

In a theoretical study, Brizuela et al. predicted weak physisorption on a threefold hollow site for cyclopentene/Pt(111) using semiempirical molecular orbital methods based on extended Hückel theory $[72,84,85]$, contradicting the di- $\sigma$ bonding postulated by Avery. For Cp, however, calculations showed better agreement with Avery's experimental results, predicting strong chemisorption in a flat-lying configuration.

To improve the understanding of the bonding of cyclopentene, Becker et al. investigated the adsorption of cyclopentene on $\mathrm{Pt}(111)$ and two surface alloys, $\mathrm{Pt}_{3} \mathrm{Sn} / \mathrm{Pt}(111)$ and $\mathrm{Pt}_{2} \mathrm{Sn} / \mathrm{Pt}(111)$ in a combined experimental and theoretical study [86]. The adsorption behaviour on $\mathrm{Pt}(111)$ reported by Avery was confirmed, showing dehydrogenation to $\mathrm{Cp}$. On both surface alloys, however, the authors report fully reversible adsorption of cyclopentene. While a $\mathrm{Pt}-\mathrm{H}$ bond promotes dehydrogenation of cyclopentene on clean $\mathrm{Pt}(111)$, no such bond is formed on either alloy, thus making desorption more favourable 
than decomposition. On all three surfaces, di- $\sigma$ bonding on bridge sites was found for cyclopentene, supporting Avery's results and contradicting the work by Brizuela et al.

Furthermore, Cp adsorption was investigated on nickel surfaces, in particular on $\mathrm{Ni}(100)$ and $\mathrm{Ni}(111)$. Using semiempirical molecular orbital methods, Comelli et al. determined that $\mathrm{Cp}$ adsorbates adsorb preferably on fourfold hollow sites on $\mathrm{Ni}(100)$ and threefold hollow sites on $\mathrm{Ni}(111)$ [87]. On both surfaces, the $\mathrm{Cp}$-metal bond is very strong, resulting in chemisorption. In similar calculations, Brizuela et al. later confirmed threefold hollow sites as the preferred adsorption sites for $\mathrm{Cp} / \mathrm{Ni}(111)[88,89]$.

Netzer et al. studied $\mathrm{Cp} / \mathrm{Rh}(111)$ using angle-resolved ultraviolet photoemission spectroscopy (ARUPS), TDS, LEED and work function measurements [90]. They reported dehydrogenation of cyclopentadiene as well as cyclopentene to $\mathrm{Cp}$ upon adsorption at room temperature. In contrast, cyclopentene forms a stable monolayer when dosed at lower temperature (between $140 \mathrm{~K}$ and $150 \mathrm{~K}$ ), a species which dehydrogenates to cyclopentadiene at $190 \mathrm{~K}$ but does not dehydrogenate further to form Cp. In a more detailed study of the adsorbed Cp species, the authors report $\pi$-bonding with the molecular plane parallel to the surface [91].

\subsubsection{Metallocene adsorption}

The adsorption of metallocenes on metal surfaces has found increasing interest due to the potential these adsorbates show as precursors for molecular electronics, in particular the formation of magnetic features [92,93]. Molecular adsorption of ferrocene, nickelocene and cobaltocene has been observed on substrates such as $\mathrm{Ag}(100), \mathrm{Cu}(100), \mathrm{Mo}(112)$ and graphite [93]. However, some metal substrates promote the decomposition of metallocenes upon adsorption; two typical studies are summarised below. To the best of the knowledge of the author, no evidence for decomposition to form Cp upon adsorption of metallocenes on metal surfaces has been reported.

Choi et al. investigated the adsorption of cobaltocene on $\mathrm{Cu}(111)$ in a photoemission study [93]. They found that upon initial adsorption, cobaltocene decomposes on the surface, while molecular adsorption occurs for exposures $\geq 14$ Langmuirs. The nature of the decomposition products has not been specified by the authors.

In a scanning tunnelling microscopy (STM) study, Braun et al. studied the thin film growth of ferrocene on $\mathrm{Au}(111)$ below $250 \mathrm{~K}$ [94]. Ferrocene was found to adsorb 
dissociatively and form a two layer structure on $\mathrm{Au}(111)$ with a first layer of close-packed upright-standing ferrocene and $\mathrm{Cp}-\mathrm{Fe}$ complexes and a second layer formed by flat-lying Cp rings which are highly mobile even at $80 \mathrm{~K}$ and diffuse to form a regular overlayer on top of the layer of ferrocene molecules.

To summarise, Cp readily forms upon adsorption of cyclopentane, cyclopentene or cyclopentadiene on many surfaces, yet not upon metallocene adsorption. Cp is typically found to adsorb with the molecular ring parallel to metal surfaces, forming $\pi$-bonding analogous to the bonding found in metallocene complexes.

\subsection{Experimental methods}

All experiments presented in this chapter were performed using the Cambridge HeSE spectrometer (cf. Chapter 2), using an incident helium beam energy between $7.5 \mathrm{meV}$ and $8.1 \mathrm{meV}$. A Cu(111) single crystal (Surface Preparation Laboratory, Netherlands) was mounted in an ultra-high vacuum (UHV) chamber with a base pressure of $3 \cdot 10^{-11}$ mbar. In order to clean the crystal, surface contaminations were first removed by argon ion sputtering $(800 \mathrm{eV}, 10 \mu \mathrm{A}, 10 \mathrm{~min}, 300 \mathrm{~K}$ ), followed by a 30 second annealing step to a temperature of $800 \mathrm{~K}$, i.e. above the $\mathrm{Cu}(111)$ roughening transition temperature. The specular helium reflectivity was used as a measure of the cleanliness of the crystal surface, which was $\geq 25 \%$. The crystal was aligned using the known diffraction pattern of a CO overlayer [27].

The dosing procedure for cyclopentadienyl was adopted from reference [79]. Since $\mathrm{Cp}$ is not a stable molecule in the gas phase, cyclopentadiene $(\mathrm{CpH})$ was dosed, which dehydrogenates to form cyclopentadienyl on the surface. Due to the setup of the Cambridge HeSE apparatus, where the mass spectrometer cannot be positioned close to the sample surface, no TDS measurements were performed to study the reaction pathway after dosing. However, comparison with the adsorption behaviour on $\mathrm{Cu}(100)$ as described in the previous section [79] as well as the results from DFT calculations performed by Sacchi and Jenkins [95], suggest that dehydrogenation of cyclopentadiene to Cp occurs on $\mathrm{Cu}(111)$. For HeSE experiments, $\mathrm{CpH}$ was dosed at $300 \mathrm{~K}$, which is above the desorption temperature for hydrogen on this substrate [39] and thus allows the study of $\mathrm{Cp}$ adsorbate motion in the absence of adsorbed hydrogen. Cyclopentadiene was obtained from distillation of its stable dimer and subsequently stored in liquid nitrogen $(77 \mathrm{~K})$ to avoid spontaneous dimerisation [96]. In order to dose the monomer, it was warmed to 
room temperature, purified by repeated freeze-thaw cycles in high vacuum to remove volatile contaminants, and immediately evaporated and dosed at $300 \mathrm{~K}$ by backfilling the UHV chamber accommodating the $\mathrm{Cu}(111)$ crystal. The dosing process was monitored via the specular helium reflectivity and the resulting uptake curves were recorded. The Cp adsorbate layer was found to be stable for up to 24 hours. Cp adsorption/desorption is not fully reversible on $\mathrm{Cu}(111)$, and the adsorbate decomposes at temperatures above $500 \mathrm{~K}$, therefore the sample was cleaned by sputtering and annealing before each new dosing process.

\subsection{Adsorption behaviour}

In this section, the diffraction and uptake behaviour of $\mathrm{Cp} / \mathrm{Cu}(111)$ is investigated to gain insight into the nature of the adsorbate species on the surface and to determine the coverage used during dynamics measurements.

The intensity of the specular helium signal was monitored during $\mathrm{Cp}$ adsorption at two different temperatures, shown in Figure 5.2. The blue line represents an uptake curve at $135 \mathrm{~K}$, where the specular reflectivity drops quickly to the background count level (originating from residual HD gas in the detector chamber). The fact that the count rate does not recover at higher exposure implies that the adsorbates remain disordered at high coverages, most likely forming irregular multilayers at low temperature. A typical uptake curve recorded at $300 \mathrm{~K}$ is shown as a red line in Figure 5.2. Dosing was performed to a specular attenuation of 2.75 , the coverage used in the dynamics measurements. A comparison between the curves at the two different temperatures suggests that the adsorption process is slower at higher temperature, which could be due to a change in the sticking coefficient with temperature.

Using Equation 2.5, the uptake curves can be used to estimate the adsorbate coverage corresponding to a certain specular attenuation. On a $\mathrm{Cu}(111)$ surface, the substrate atom density is $n_{s}=0.18 \AA^{-2}$. The scattering cross section for Cp can be estimated as approximately $200 \AA^{2}$ by comparing values determined for other adsorbate species (e.g. $114 \AA^{2}$ for $\mathrm{C}_{2} \mathrm{H}_{4} / \mathrm{Cu}(100)$ or $143 \AA^{2}$ for $\left.\mathrm{CO} / \mathrm{Cu}(111)\right)[15,27]$ ). The coverage corresponding to a specular attenuation of 2.75 is thus approximately 0.03 monolayers (ML), where a coverage of $1 \mathrm{ML}$ is defined as one adsorbate particle per substrate atom. Another more accurate determination of the coverage, presented in Section 5.7, confirms a coverage of $0.03 \mathrm{ML}$. 


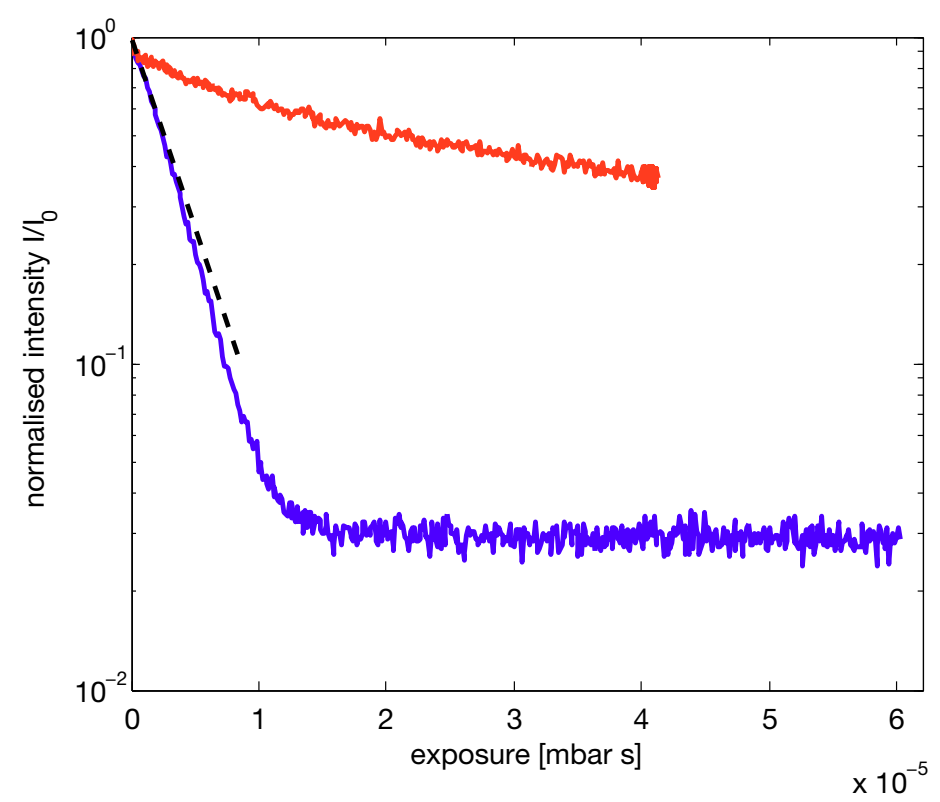

Figure 5.2: Uptake curves for $\mathrm{Cp}$ on $\mathrm{Cu}(111)$ at $135 \mathrm{~K}$ (blue) and $300 \mathrm{~K}$ (red) measured on the HeSE spectrometer. The dose at $300 \mathrm{~K}$ is to a specular attenuation of 2.75 , the coverage used in the HeSE measurements.

Furthermore, uptake curves provide information about the interactions between adsorbate particles (cf. Chapter 2) [15]. The uptake curve for $\mathrm{Cp} / \mathrm{Cu}(111$ ) at $135 \mathrm{~K}$ (cf. Figure 5.2) is approximately linear with a slight downwards trend at the beginning of the curve (compare with straight dashed line), implying that $\mathrm{Cp}$ adsorbates interact weakly repulsively at low coverages.

Diffraction scans for $0.03 \mathrm{ML} \mathrm{Cp} / \mathrm{Cu}(111)$ along two crystal azimuths, $<1 \overline{1} 0>$ and $<11 \overline{2}>$, are shown in Figure 5.3. For comparison, a scan of the clean $\mathrm{Cu}(111)$ surface along $<11 \overline{2}>$ is shown. Both curves along $<11 \overline{2}>$ show a diffraction peak at $2.84 \AA^{-1}$, corresponding to the lattice constant on this substrate [40]. During the course of this work, different $\mathrm{Cu}(111)$ crystals have been used. Typically, a pristine $\mathrm{Cu}(111)$ crystal does not show a diffraction peak, however, a small peak can become apparent as the crystal ages, implying that the current crystal may contain an extremely small number of defects, forming a lattice gas. A newer $\mathrm{Cu}(111)$ crystal used for the experiments presented in Chapter 6 did not show the same effect (cf. for example Figure 6.4). The increase in peak height after adsorption of $\mathrm{Cp} / \mathrm{Cu}(111)$ could be due to $\mathrm{Cp}$ forming a lattice gas on the surface. However, further investigation would be required to rule out slight misalignment of the crystal direction, which could also explain the difference in peak height. 


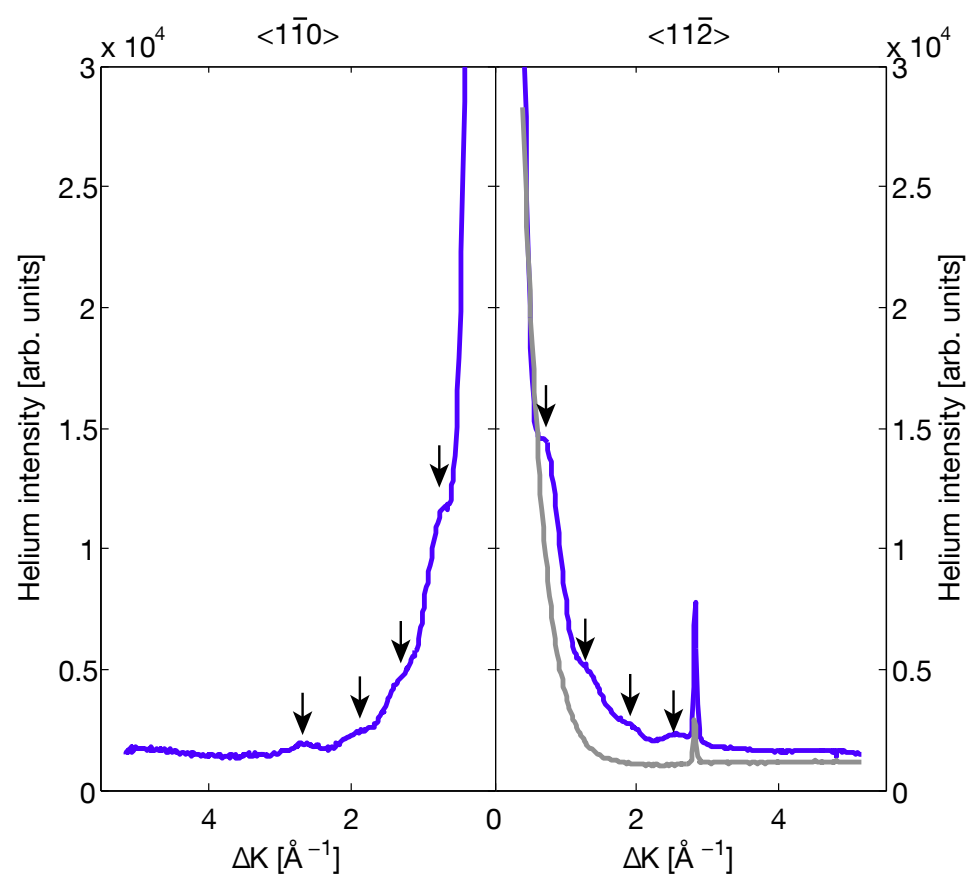

Figure 5.3: Diffraction scans of $\mathrm{Cp} / \mathrm{Cu}(111)$ at $300 \mathrm{~K}$ along two azimuths. The blue lines show the diffraction pattern of $0.03 \mathrm{ML} \mathrm{Cp} / \mathrm{Cu}(111)$, exhibiting several diffraction rings (arrows) and a diffraction peak. A diffraction scan of the clean $\mathrm{Cu}(111)$ substrate is shown as a grey line for comparison.

In addition to the diffraction peak, several diffraction rings can be observed at $0.7 \AA^{-1}$, $1.3 \AA^{-1}, 1.9 \AA^{-1}$ and $2.6 \AA^{-1}$ (arrows). From these scans alone, it is difficult to distinguish between form factor and structure factor rings; coverage dependent data would be needed. However, Section 5.8 will show that neither of the ring positions corresponds to the expected structure factor ring for a coverage of 0.03 ML. Therefore, these features are likely due to scattering from the $\mathrm{Cp}$ molecule, not the ordering of the overlayer, implying that they are form factor rings.

To summarise, the coverage used in HeSE experiments is approximately 0.03 ML, which will be confirmed by a more accurate estimate in Section 5.8. Uptake curves show weak repulsive forces between adsorbate molecules, while form factor rings and a diffraction peak caused by lattice gas are apparent in diffraction scans. 


\subsection{Measuring the surface dynamics}

The surface dynamics of $0.03 \mathrm{ML} \mathrm{Cp} / \mathrm{Cu}(111)$ are investigated as a function of temperature, momentum transfer and crystal direction. In this section, the lineshapes of the intermediate scattering function (ISF) measured by HeSE are presented in order to determine which functional form best represents the data. Consequently, the momentum transfer dependence of the dephasing rate is discussed and an apparent activation energy for diffusion deduced from temperature dependent data.

\subsubsection{Multi-component lineshapes}

HeSE measurements typically show surface motion on a range of timescales, comprising different forms of substrate and adsorbate motion. Typical curves for the motion of $\mathrm{Cp} / \mathrm{Cu}(111)$ at $300 \mathrm{~K}$ at small and large momentum transfers along two crystal directions are displayed as blue dots in Figure 5.4. To illustrate the different processes occuring on a range of different timescales, the polarisation curves are presented on a logarithmic time axis. Two main features can be identified in the polarisation curves: periodic oscillations at small times (cf. top two panels) and a decay with spin-echo time (represented by the black line) which occurs over several orders of magnitude and is the dominant feature in all curves. These two features are discussed separately below. In addition, a constant offset from zero to which the decay levels off to can be observed (cf. for example on the right-hand side in the bottom left panel). The constant level is typically due to scattering from static defects on the surface [6]. The variation of the intensity of the static level with momentum transfer is a diffraction pattern.

At small momentum transfer values $\left(\Delta \boldsymbol{K} \leq 1.2 \AA^{-1}\right)$, oscillatory features indicate the presence of vibrational motion. To separate all frequencies present and identify these vibrations, the spectra have been Fourier transformed into energy space and the variation of the peak positions with $\Delta \boldsymbol{K}$ studied, as shown in Figure 5.5. Four vibrational modes are observed in the $\mathrm{Cp} / \mathrm{Cu}(111)$ spectra, three of which can be assigned as described below. The strongest features are two dispersive modes, presented as red and blue crosses. Harten et al. have reported two dispersive modes in phonon measurements of the clean $\mathrm{Cu}(111)$ surface using quasi-elastic helium atom scattering [40], which was later confirmed by Mohamed et al. in an electron energy loss spectroscopy (EELS) study [97]. Comparing the strong dispersive modes in the $\mathrm{Cp} / \mathrm{Cu}(111)$ data with the published phonon modes of clean $\mathrm{Cu}(111)$ [40], shown as black lines in Figure 5.5, the oscillations 
in the ISFs can be attributed primarily to substrate phonons. A small shift to lower energies is observed, indicating an increased effective mass of the copper atoms upon $\mathrm{Cp}$ adsorption, decreasing the vibrational frequency. The dispersion curve shown in blue exhibits two kinks (cf. arrows in Figure 5.5) along both azimuths, which are most likely caused by resonances of the substrate phonon with $\mathrm{Cp}$ vibrational modes, as discussed in Section 5.9. In addition, two non-dispersive modes at $-1.9 \mathrm{meV}$ and $-4.6 \mathrm{meV}$, which are only weakly apparent in the data, are observed. The energy loss mode at approximately $-4.6 \mathrm{meV}$ is at the same location as the first kink in the blue dispersion curve, and agrees well with the energy for the Cp T-mode (horizontal vibrational mode)
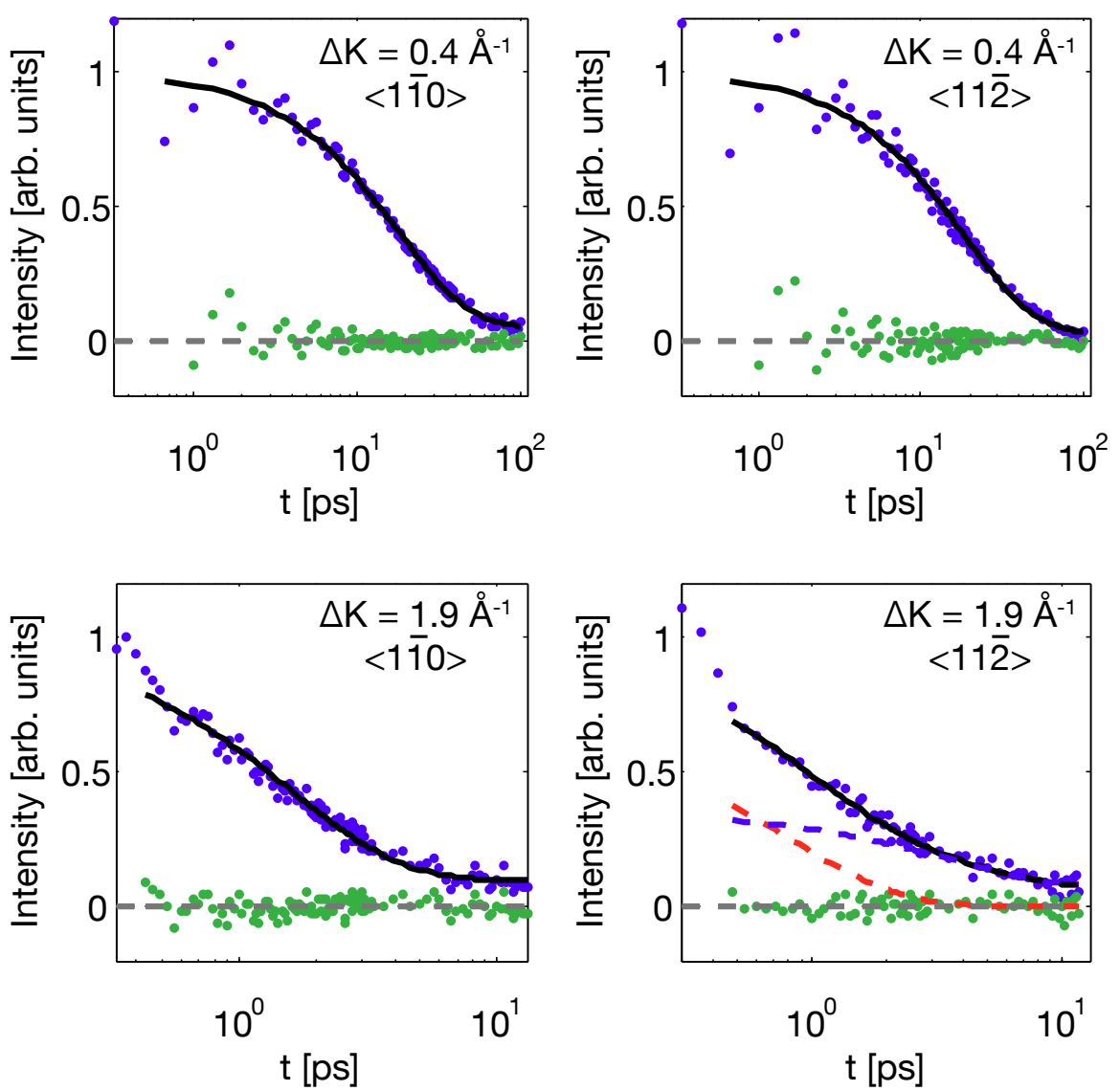

Figure 5.4: Polarisation measurements as a function of spin-echo time for $0.03 \mathrm{ML} \mathrm{Cp} / \mathrm{Cu}(111)$. The top row shows data at $0.4 \AA^{-1}$, the bottom row at $1.9 \AA^{-1}$. Experimental data are plotted as blue points, an exponential is shown in black and the residual after subtracting the exponential from the data as green points. The blue and red dashed curves in the bottom right panel illustrate the two exponents composing the black line in that panel. 


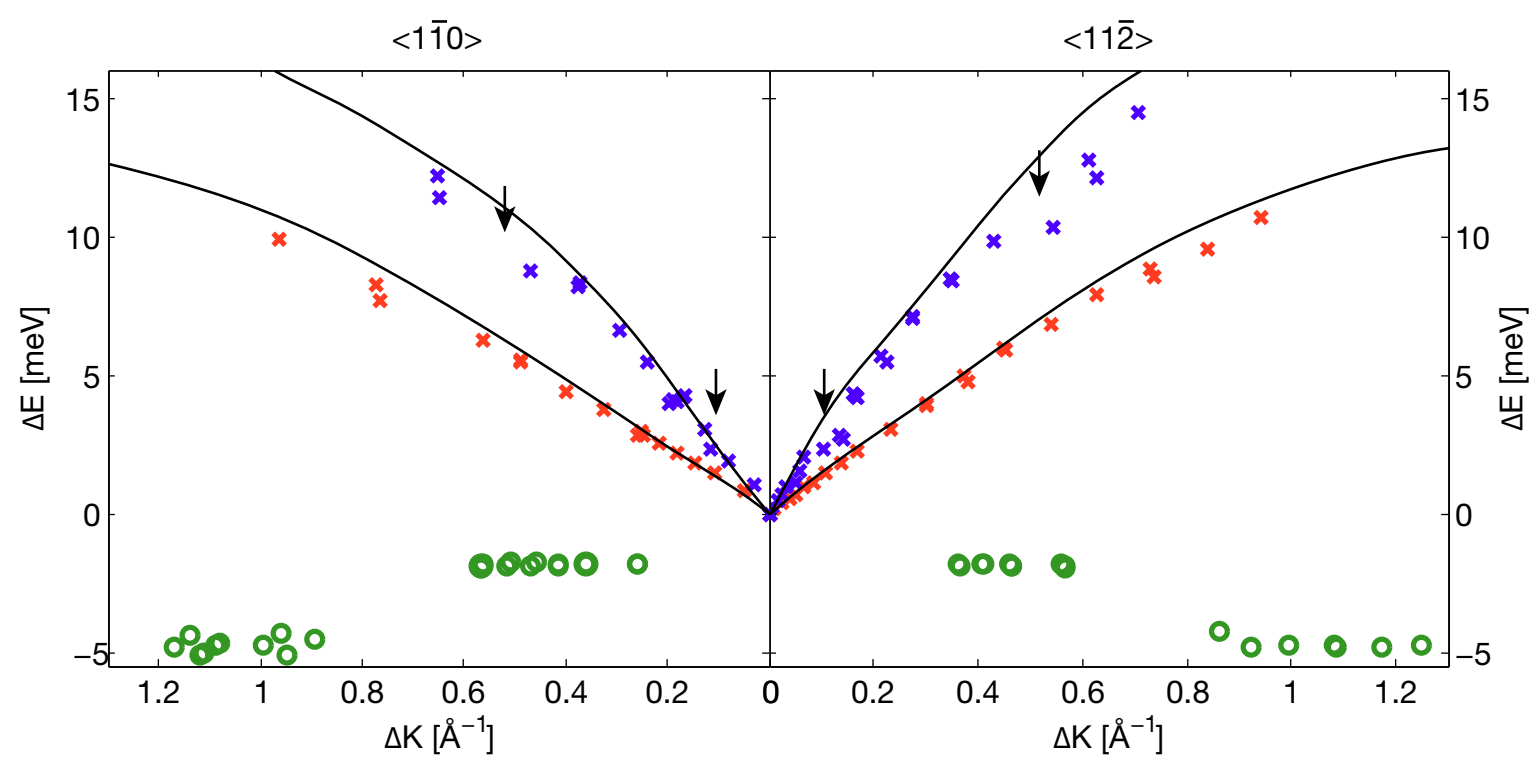

Figure 5.5: Analysis of the vibrational modes of $\mathrm{Cp} / \mathrm{Cu}(111)$ at $300 \mathrm{~K}$. Two dispersive modes are shown in blue and red, which can be identified as substrate phonons, in good agreement with the results published by Harten et al. for the clean $\mathrm{Cu}(111)$ substrate, which are reproduced as black lines [40]. A shift to lower energies results from the increased effective mass of the substrate atoms upon Cp adsorption and two kinks (arrows) in the higher energy mode are most likely due to resonances with $\mathrm{Cp}$ vibrations. Two additional non-dispersive modes, which are only weakly apparent in the experiment, are presented in green.

which is estimated in Section 5.9. In the analysis of Cp diffusion, the oscillatory features are disregarded.

The dominant, feature in the ISFs presented in Figure 5.4 is the decay of polarisation with spin-echo time, $t$. The decaying component can be separated into two processes that occur on very different timescales. At long spin-echo times, a slow decay is seen in all spectra, described by the black lines in Figure 5.4. In addition, a fast decay at short times, $t$, becomes apparent in the bottom panels in Figure 5.4 (cf. blue dots left of the black lines). As they occur on such different timescales, the two decays can be analysed more or less independently. The fast decay is found to occur at times approximately below $0.4 \mathrm{ps}$ and its intensity increases with momentum transfer. It can be attributed to diffusion of the adsorbate particles within the adsorption well, termed intracell diffusion [98]. In Section 5.9, a brief analysis of the intracell diffusion is presented.

This chapter predominantly treats the motion on longer timescales which arises from diffusion on larger length scales, i.e. "inter-cell" diffusion. Since the intra- and inter-cell 
diffusion occur on such vastly different timescales, the slower decay can be investigated individually simply by disregarding the data $<0.4 \mathrm{ps}$, thus isolating the slow component. A determination of the friction coefficient using molecular dynamics (MD) simulations (cf. Section 5.6) demonstrates that 0.4 ps is the correct cut-off value to use as it corresponds to the friction that dominates the motion within the adsorption site. In order to study the slow decay, it is typically described by a function of the form $f(t)=a \cdot \exp (-\alpha t)+c$, where $a$ is the preexponential factor, $c$ the static component, and the decay constant, $\alpha$, the dephasing rate.

Measurements of $0.03 \mathrm{ML} \mathrm{Cp} / \mathrm{Cu}(111)$ at momentum transfer values below $0.6 \AA^{-1}$ can be described by one single exponential function as shown by the black line in the graphs in the top row of Figure 5.4. The green dots show the residual after subtracting the exponential from the data, demonstrating that the black line represents the data well as it does not deviate systematically from zero (apart from the oscillations due to phonons). At larger momentum transfer, the behaviour along the two azimuths becomes different. Along $<1 \overline{1} 0>$, all measured polarisation curves appear as a simple exponential function, as illustrated in the bottom left panel in Figure 5.4. In the other crystal direction, $<11 \overline{2}>$, however, a single exponential is not sufficient to describe the data. Instead, a sum of two exponentials, of the functional form

$$
f(t)=a_{1} \cdot \exp \left(-\alpha_{1} t\right)+a_{2} \cdot \exp \left(-\alpha_{2} t\right)+c
$$

is required to describe the data fully, shown as red and blue dashed lines in the bottom right panel which add up to the solid black line. The residual after subtracting both exponentials shows no systematic deviation from zero, confirming that a double decay represents the experimental data well.

The differing behaviour between the two principal crystal directions - single exponential along $<1 \overline{1} 0>$ versus sum of two exponentials along $<11 \overline{2}>-$ is characteristic for hopping on a lattice of degenerate fcc and hcp hollow sites on an fcc(111) surface, as described in detail in Section 2.4.1. In fact, it is the only model predicting a double decay along $<11 \overline{2}>$ and a single exponential along $<1 \overline{1} 0>$. With an increasing energy difference between fcc and hcp hollow sites, a second decay along $<1 \overline{1} 0>$ should become apparent, gaining in intensity as the energy difference increases. The ISFs for $\mathrm{Cp} / \mathrm{Cu}(111)$, however, appear as a single decay along this crystal direction, indicating that the two kinds of hollow sites exhibit similar adsorption energies. In Section 5.8, a method of analysing all data combined in a more rigorous way, rather than each ISF individually, is 
employed to determine accurately the energy difference between the two kinds of hollow sites, confirming that it is indeed small.

\subsubsection{Jump diffusion curves}

The previous section demonstrated how the shape of the ISFs alone gives a strong indication of the preferred adsorption site for an adsorbate on an fcc(111) surface. The characteristic signatures for approximately degenerate fcc and hcp hollow sites is observed for $\mathrm{Cp} / \mathrm{Cu}(111)$, with a single decay along the $<1 \overline{1} 0>$ direction and a sum of two exponential functions at certain $\Delta \boldsymbol{K}$ values along $<11 \overline{2}>$. The momentum transfer dependence of the decays confirms the adsorption site and provides further information about the hopping motion and the inter-adsorbate forces.

All data along the $<1 \overline{1} 0>$ direction and data in the momentum transfer region below $0.7 \AA^{-1}$ along $<11 \overline{2}>$ appears as a single exponential function. Adding a second decay, as described in Equation 5.1, does not improve the description of the ISFs in this region. The prefactor $a_{2}$ is thus zero for these data. At momentum transfers greater than $0.7 \AA^{-1}$ along $<11 \overline{2}>$, however, a sum of two exponentials is required to describe the ISFs fully, i.e. $a_{2} \neq 0$. The analytical model for hopping between degenerate fcc and hcp sites predicts the relative magnitude and intensity of the fast decay with respect to the slow decay, defining the ratios of prefactors, $a_{1} / a_{2}$, and exponents, $\alpha_{1} / \alpha_{2}$, in Equation 5.1 which can be used to reduce the number of free parameters in Equation 5.1 from five to only three.

The resulting $\alpha(\Delta \boldsymbol{K})$ curves for $\mathrm{Cp}$ diffusion at $300 \mathrm{~K}$ and $135 \mathrm{~K}$ are shown in Figures 5.6 and 5.7, respectively. The $\alpha_{1}$ and $\alpha_{2}$ values are shown as blue and red points, respectively. In addition, the curves predicted by the analytical model for hopping on degenerate fcc and hcp sites are shown as solid lines, scaled to match the experimental results. The dashed line represents a decay predicted to have less than $1 \%$ intensity. At $300 \mathrm{~K}$, excellent agreement between model and experiment is found, confirming hollow sites as the preferred adsorption sites. The data for $135 \mathrm{~K}$ also show reasonable agreement with the analytical model for hopping between degenerate fcc and hcp sites which is thus a close description of the data. A small asymmetry of the curves at $135 \mathrm{~K}$, with both $\alpha$ values consistently too high at small momentum transfer and too low at large values of $\Delta \boldsymbol{K}$, can be attributed to a small energy difference between fcc and hcp sites, as discussed in Section 5.8 . 


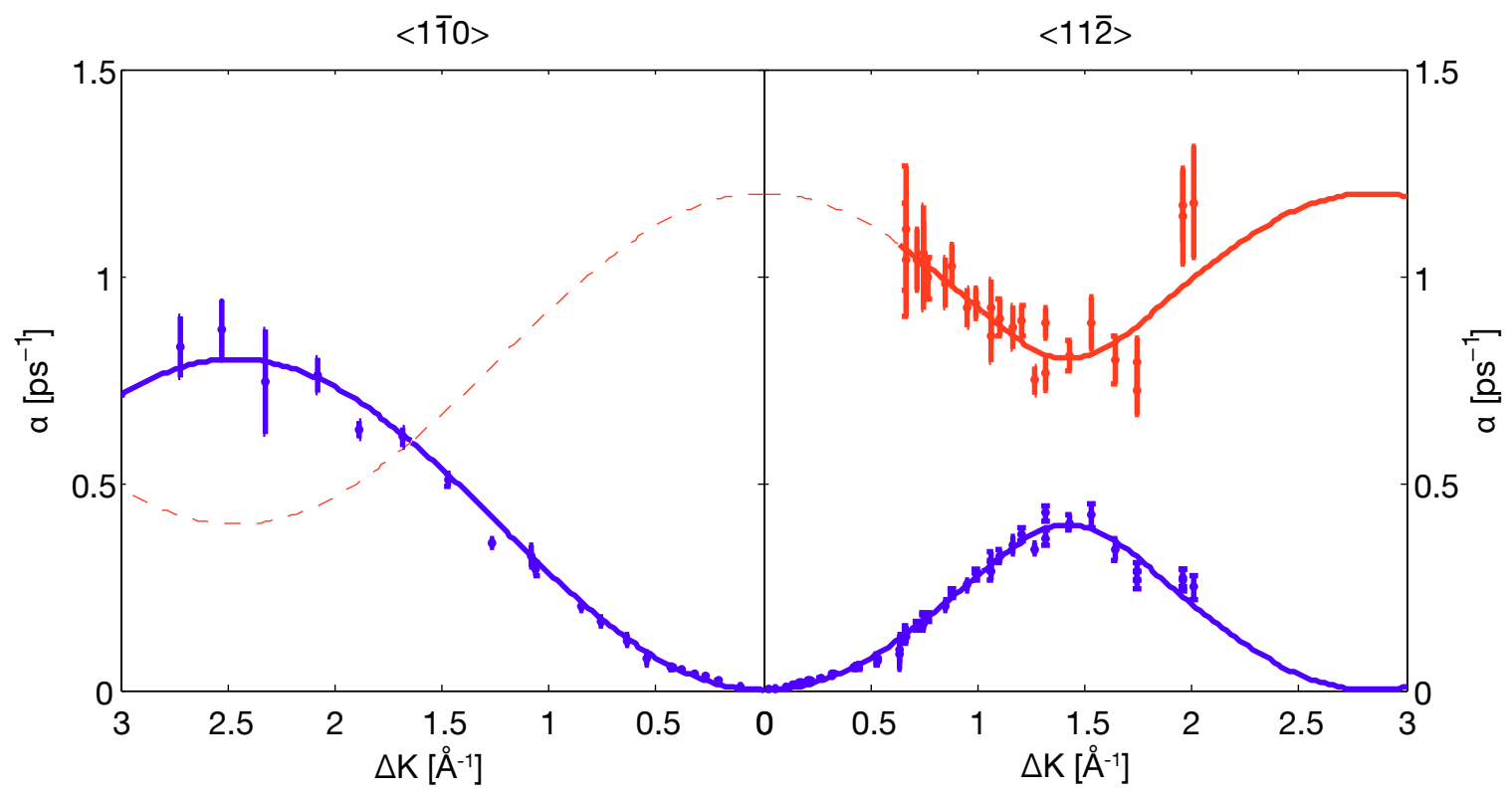

Figure 5.6: $\alpha(\Delta \boldsymbol{K})$ curves for $\mathrm{Cp} / \mathrm{Cu}(111)$ at $300 \mathrm{~K}$, comparing experimental data at $0.03 \mathrm{ML}$ with the analytical model for single jumps between degenerate hollow sites. Along $<1 \overline{1} 0>$ a single curve is observed whereas the ISFs at certain momentum transfers in the $\langle 11 \overline{2}>$ direction show two decays. The solid lines present the analytical model for single jumps between hollow sites scaled to match the data; the dashed lines illustrate predicted decays with less than $1 \%$ intensity.

In addition, the purely sinusoidal shape of the curves indicates that Cp hops predominantly between nearest neighbour sites since double jumps would add shorter sinusoids, changing the shape of the curves [6]. A small proportion of double jumps indicates a high friction between adsorbate and substrate. Studying the low momentum transfer region of the $\mathrm{Cp} / \mathrm{Cu}(111) \alpha(\Delta \boldsymbol{K})$ curves, no significant deviation from a sinusoidal form is found, suggesting that $\mathrm{Cp}$ adsorbates do not repel each other strongly (compare to Figure 2.17). Such weak lateral interaction forces are rather unexpected for an ionic adsorbate. Alkali metals, adsorbates of similar mass and dipole moment, show prominent de Gennes features at comparable coverages [99, 100].

Finally, to study the coverage dependence of $\mathrm{Cp}$ diffusion on $\mathrm{Cu}(111)$, polarisation curves were recorded for varying coverage at a fixed momentum transfer of $0.4 \AA^{-1}$ at $300 \mathrm{~K}$. In the coverage range accessible without overpressure in the sample chamber (cf. $300 \mathrm{~K}$ uptake curve in Figure 5.2), no change in the jump rate is observed, indicating that $\mathrm{Cp}$ diffusion is coverage independent. 


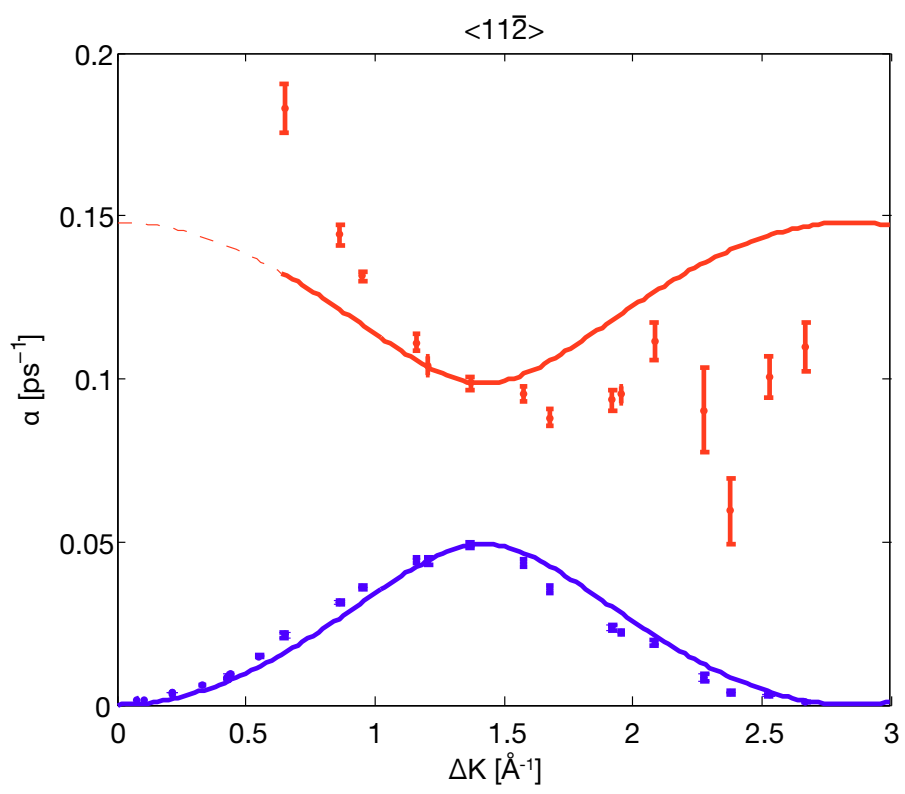

Figure 5.7: $\alpha(\Delta \boldsymbol{K})$ curves for $\mathrm{Cp} / \mathrm{Cu}(111)$ at $135 \mathrm{~K}$ along $<11 \overline{2}>$, comparing experimental data at $0.03 \mathrm{ML}$ with the analytical model for single jumps between hollow sites. At momentum transfers greater than $0.7 \AA^{-1}$ two decays are observed in the ISFs. The solid lines present the analytical model for single jumps between hollow sites scaled to match the data; the dashed line illustrates predicted decays with less than $1 \%$ intensity.

\subsubsection{An activation energy from temperature dependence}

In order to obtain further information about Cp diffusion on fcc and hcp sites on $\mathrm{Cu}(111)$, temperature dependent measurements at a fixed value in $\Delta \boldsymbol{K}$ have been performed. For thermally activated processes, Arrhenius' law predicts the temperature dependence of the dephasing rate, $\alpha$, as

$$
\alpha=\alpha_{0} \cdot \exp \left(-\frac{E_{a}}{k_{B} T}\right)
$$

where $\alpha_{0}$ is the pre-exponential factor describing the jump attempt frequency, $E_{a}$ is the activation energy for diffusion, $k_{B}$ the Boltzmann constant and $T$ the temperature of the sample surface. Taking the natural logarithm of Equation 5.2 results in

$$
\ln \alpha=-\frac{E_{a}}{k_{B} T}+\ln \alpha_{0}
$$


a linear relationship between the inverse of the temperature, $1 / T$, and the natural logarithm of the dephasing rate, $\ln \alpha$. Plotting $1 / T$ versus $\ln \alpha$ gives a straight line with gradient $-E_{a} / k_{B}$ and intercept $\ln \alpha_{0}$.

Figure 5.8 presents Arrhenius plots for $0.03 \mathrm{ML} \mathrm{Cp} / \mathrm{Cu}(111)$. At $0.4 \AA^{-1}$, shown in the main panel, measurements along $<11 \overline{2}>$ have been recorded in a temperature range between $111 \mathrm{~K}$ and $400 \mathrm{~K}$. In order to study the azimuthal dependence of the Arrhenius activation energy, measurements along $<1 \overline{1} 0>$ were recorded between $200 \mathrm{~K}$ and $300 \mathrm{~K}$. Both data sets show a linear relationship between $\ln \alpha$ and $1 / T$, confirming that the mechanism of $\mathrm{Cp}$ diffusion on $\mathrm{Cu}(111)$ does not change in the temperature range investigated here. Fitting a straight line to the more comprehensive data set along $<11 \overline{2}>$ gives an apparent energy barrier for hopping of $41 \pm 1 \mathrm{meV}$. Measurements along the orthogonal crystal direction provide an energy barrier within the error bars of this value, $E_{a}=43 \pm 2 \mathrm{meV}$, confirming that the activation barrier for diffusion and thus the diffusion mechanism are azimuthally isotropic.

In addition, further temperature dependent experiments have been performed at $1.9 \AA^{-1}$ along $<11 \overline{2}>$ (inset) to investigate the effect of the two-component lineshapes on the apparent activation barrier. Each curve consists of two exponentials, of the functional form given in Equation 5.1, which results in two Arrhenius curves. The two values for an apparent activation energy of $44 \pm 5 \mathrm{meV}$ (slow decay) and $43 \pm 9 \mathrm{meV}$ (fast decay) are both within the error bars of the activation energy found at lower momentum transfer, indicating that the activation energy is independent of $\Delta \boldsymbol{K}$ and of the precise lineshapes in the ISFs. Due to decreasing signal levels at larger momentum transfer values and the increased uncertainty from adding two additional free parameters, however, the error bars are much more pronounced over those at $0.4 \AA^{-1}$. For systems which exhibit jumping on non-Bravais lattices, it is therefore advisable to study the temperature dependent behaviour of diffusion in an area of simple single-component lineshapes.

To conclude, HeSE experiments of $0.03 \mathrm{ML} \mathrm{Cp} / \mathrm{Cu}(111)$ show that isolated adsorbate molecules hop predominantly in single jumps on a non-Bravais lattice of fcc and hcp sites. Temperature dependent measurements have provided an apparent activation energy for diffusion of $41 \pm 1 \mathrm{meV}$. No evidence of coverage dependent adsorption behaviour has been observed and evidence for strong lateral interactions amongst Cp adsorbates is missing in the HeSE data. The following sections provide further information on the microscopic details of $\mathrm{Cp}$ adsorption and the energy landscape for adsorbate diffusion using DFT calculations, MD simulations and a global Bayesian analysis, respectively. 


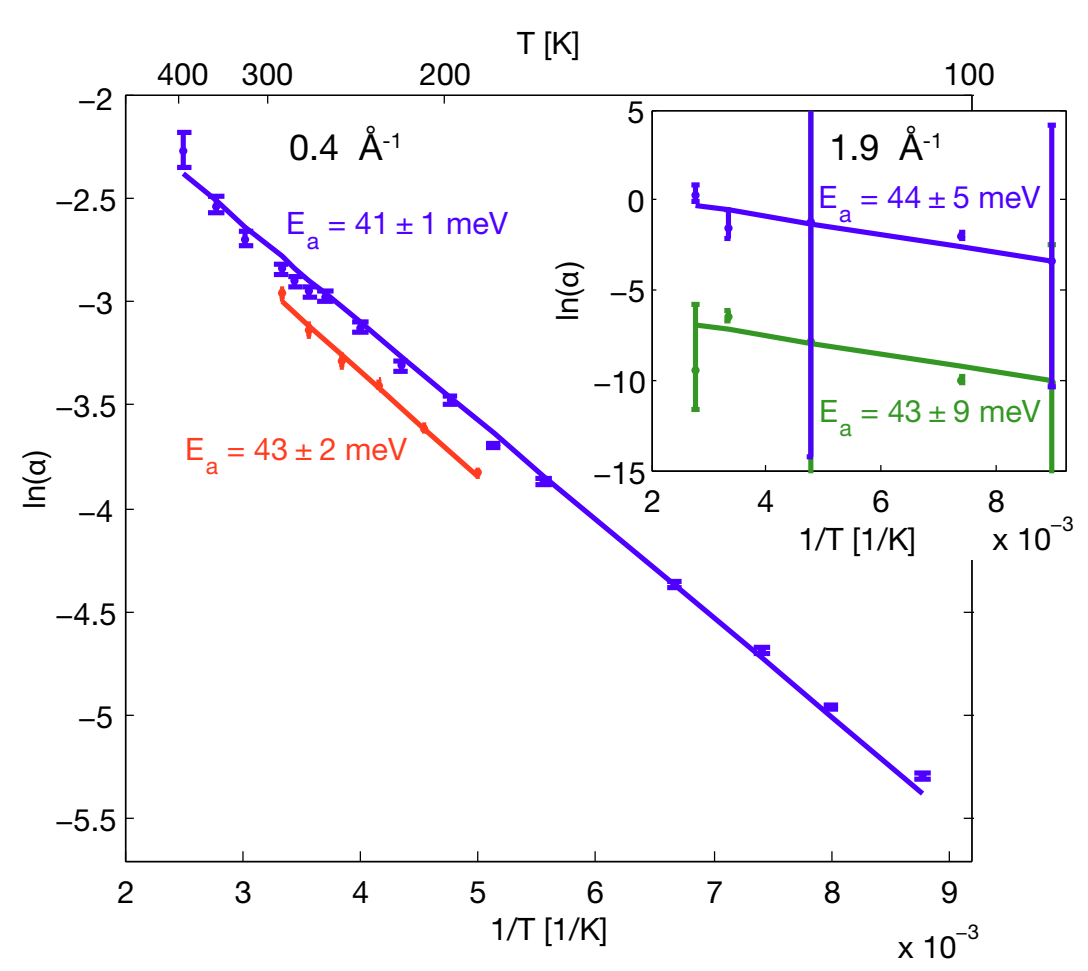

Figure 5.8: Arrhenius plots for $0.03 \mathrm{ML} \mathrm{Cp} / \mathrm{Cu}(111)$ at $0.4 \AA^{-1}$ (main panel) and $1.9 \AA^{-1}$ (inset). Experimental data are shown as points, the lines represent a linear fit to the data. At $0.4 \AA^{-1}$, good agreement between measurements along the two crystal directions $\langle 1 \overline{1} 0\rangle$ (red) and $<11 \overline{2}>$ (blue) is found. At higher momentum transfer values, $1.9 \AA^{-1}$, a sum of two exponentials results in two $E_{a}$ values along $<11 \overline{2}>$, both within error bars of the data at lower $\Delta \boldsymbol{K}$.

\subsection{First principles density functional theory calculations}

To complement the HeSE data presented above, Sacchi and Jenkins from the Department of Chemistry at Cambridge have carried out first principles density functional theory (DFT) calculations for $\mathrm{Cp} / \mathrm{Cu}(111)[101,102]$. In this section, their main results are summarised and more comprehensive conclusions from combined experimental and theoretical findings are drawn.

Various binding sites, adsorption geometries, and orientations were investigated using the CASTEP code [103]. Electronic exchange and correlation were included through the generalised gradient approximation (GGA) using the Perdew Wang (PW91) exchangecorrelation functional [104]. It was found that, in this particular case, the van der Waals 
dispersion forces could be neglected due to predominantly ionic and covalent bonding. In contrast, two examples where dispersion forces dominate the bonding are discussed in Chapters 6 and 7 .

According to first principles calculations, Cp adsorbs with the molecular ring parallel to the surface for all high symmetry sites on the substrate. While the carbon ring remains planar, the $\mathrm{C}-\mathrm{H}$ bonds tilt away from the surface at an angle of around $10^{\circ}$. The bond lengths of $\mathrm{Cp}$ are within error bars of the ones determined by X-ray diffraction (cf. Section 5.1) [71]. The adsorption energies for the four high symmetry sites on $\mathrm{Cu}(111)$ are shown in Table 5.1 for a $(2 \sqrt{3} \times 2 \sqrt{3})$ R30 unit cell. The two kinds of hollow sites, fcc and hcp, are the most favourable adsorption sites, while top sites have the lowest adsorption energy. Only a slight decrease in the adsorption energies is seen with increasing coverage.

\begin{tabular}{c|cccc}
\hline adsorption site & fcc hollow & hcp hollow & bridge & top \\
\hline adsorption energy $[\mathbf{e V}]$ & 1.732 & 1.732 & 1.671 & 1.198 \\
\hline
\end{tabular}

Table 5.1: Adsorption energies for $\mathrm{Cp} / \mathrm{Cu}(111)$ in a $(2 \sqrt{3} \times 2 \sqrt{3}) \mathrm{R} 30^{\circ}$ unit cell from DFT calculations (from $[101,102]$ ).

Two different rotational configurations on an fcc site showed an energy difference of around $30 \mathrm{meV}$ to $50 \mathrm{meV}$, suggesting that rotational motion should occur at the temperatures at which the present experiments were performed. However, due to the rotationally symmetric appearance of $\mathrm{Cp}$ in a helium beam, no signatures for rotational motion have been observed experimentally. Further calculations searching the transition state for diffusion of $\mathrm{Cp}$ provided an energy barrier on bridge sites, between adjacent hollow sites, of $55 \mathrm{meV}$. This value is comparable with the experimentally determined apparent activation barrier of $41 \pm 1 \mathrm{meV}$.

Having determined the preferred adsorption site experimentally and theoretically, MD simulations can be performed to get a more complete picture of the potential energy surface (PES) and to determine a friction coefficient. In order to model the interaction between adsorbates, DFT calculations of the dipole moment and work function change were performed. The calculated dipole moment and work function changes are summarised in Table 5.2 [101,102]. Assuming predominantly dipolar pair-wise interactions, the Topping model relates the work function change determined by DFT calculations to an estimated 
dipole force $[105,106]$. The model describes the work function change as

$$
\Delta \Phi=\frac{p_{0} n_{0} \Theta}{\epsilon_{0}\left[1+9 \alpha_{p}\left(n_{0} \Theta\right)^{3 / 2}\right]}
$$

where $\Theta$ is the adsorbate coverage, $n_{0}$ the adsorbate density at $\Theta=0$, $\epsilon_{0}$ the vacuum permittivity, $p_{0}$ the dipole moment in the zero coverage limit, and $\alpha_{p}$ the polarisability. The results in Table 5.2 give $p_{0}=2.02$ Debye and polarisability $\alpha_{p}=23.3 \AA^{3}$ for $\mathrm{Cp} / \mathrm{Cu}(111)$. From the two parameters $\alpha_{p}$ and $p_{0}$, the dipole moment at any given coverage can be calculated as

$$
p=\frac{p_{0}}{\left.1+9 \alpha_{p}\left(n_{0} \Theta\right)^{3 / 2}\right)}
$$

This dipole moment is then used to define the interaction forces, $F_{i, j}$, in the simulation (cf. Section 5.6).

\begin{tabular}{cccc}
\hline structure & $\boldsymbol{\Theta}[\mathrm{ML}]$ & $\boldsymbol{\Delta} \boldsymbol{\Phi}[\mathrm{eV}]$ & $\boldsymbol{p}[$ Debye $]$ \\
\hline$(2 \sqrt{3} \times 2 \sqrt{3}) \mathrm{R} 30^{\circ}$ & 0.08 & -0.799 & 1.45 \\
$(3 \times 3)$ & 0.11 & -0.982 & 1.33 \\
$(\sqrt{7} \times \sqrt{7}) \mathrm{R} 19.1^{\circ}$ & 0.14 & -1.033 & 1.09 \\
\hline
\end{tabular}

Table 5.2: Work function change and dipole moment of $\mathrm{Cp} / \mathrm{Cu}(111)$ as given by DFT calculations (from [101, 102]).

DFT results show a charge transfer of 1.1 electrons from the surface to the $\mathrm{Cp}$, so that the adsorbate species resembles the aromatic anion in the gas phase. Surprisingly, however, the work function change for $\mathrm{Cp} / \mathrm{Cu}(111)$ is smaller than that observed experimentally for $\mathrm{Na} / \mathrm{Cu}(111)$ at a similar coverage [107], despite the ionic nature of the bonding. The lack of work function change can be attributed to the so-called "cushion effect" [74], describing electron depletion above the topmost surface atoms upon adsorption of the molecule. This effect creates an additional dipole, thereby reducing the work function and thus weakening the repulsive interactions between adsorbate molecules. 


\subsection{Molecular dynamics simulations}

Due to their structural rigidity, aromatic molecules are ideal candidates for centre-ofmass simulations. The motion of $\mathrm{Cp} / \mathrm{Cu}(111)$ was therefore modelled in simple classical Langevin molecular dynamics (MD) simulations, where Cp was represented by a point-like particle of mass 65 amu. Even though this is a rather crude estimate, in this particular case the simulations are found to agree extraordinarily well with the experimental data.

In Section 2.4.3, the general principle of classical 2D Langevin MD simulations to model adsorbate motion over a potential energy surface (PES) was presented [42]. The adsorbate-substrate interactions are given by a friction term, $\eta$, along with a random impulse term to ensure energy conservation, while lateral interactions are described by pairwise forces.

At the start of each simulation, 60 particles are randomly allocated and given a random kinetic energy in all directions. The system is then allowed to relax for 2000 steps before the core part of the simulation starts. Polarisation curves are calculated from the adsorbate trajectories in 0.16 ps time steps at 25 points in $\Delta \boldsymbol{K}$ space, with each ISF $480 \mathrm{ps}$ long. In order to reduce the noise level in the simulations, the average of 64 runs was taken for each simulated curve. The pair-wise inter-adsorbate interactions are defined by the low coverage limit dipole moment and polarisability obtained from DFT calculations, as presented in Section 5.5.

This section summarises the method used for creating a PES for adsorption on degenerate fcc and hcp hollow sites, then presents the best description of the $\mathrm{Cp} / \mathrm{Cu}(111)$ data and finally investigates microscopic aspects of the friction.

\subsubsection{Creating a potential energy surface}

To simulate the diffusive motion of $\mathrm{Cp} / \mathrm{Cu}(111)$, a PES with minima at fcc and hcp sites is required, with two key parameters: the curvature of the adsorption well and the energy barrier between hollow and bridge sites (the transition state). A simple method of achieving such a PES $[43,44]$ is to describe the potential landscape by a Fourier series

$$
V(\boldsymbol{r})=A_{1} \sum_{i=1}^{3} \cos \left(\boldsymbol{g}_{i} \cdot \boldsymbol{r}\right) .
$$


Three vectors $\boldsymbol{g}_{i}$ define the hexagonal $\mathrm{Cu}(111)$ geometry

$$
\begin{array}{r}
\boldsymbol{g}_{1}=(\zeta, 0), \\
\boldsymbol{g}_{2}=(\zeta \cos (\pi / 3), \zeta \sin (\pi / 3)), \\
\boldsymbol{g}_{3}=(-\zeta \cos (\pi / 3), \zeta \sin (\pi / 3)),
\end{array}
$$

where $\zeta=4 \pi /(\sqrt{3} a)$ and $a$ is the $\mathrm{Cu}(111)$ lattice constant. Since the energy difference between the two types of hollow sites was found to be small, all simulations are performed for degenerate fcc and hcp sites.

In order to alter the shape of the sinusoids connecting the different sites in the potential energy landscape without affecting the absolute energies of the different adsorption sites, the PES is first scaled so that the energy ranges from 0 to 1 , then the potential is raised to a power $p$ to change the curvature, and finally the energy range is scaled back to its original value. Changing $p$ can flatten or sharpen the adsorption well while making the transition state narrower or wider, respectively.

Hence there are two parameters which can be varied in the PES to optimise the description of the experimental data, the energy difference between hollow and bridge sites (i.e. the energy barrier for diffusion) and the curvature in the adsorption well controlled by the parameter $p$. The PES found to best reproduce the $\mathrm{Cp} / \mathrm{Cu}(111)$ data is shown in Figure 5.9, exhibiting an energy barrier of $40 \pm 3 \mathrm{meV}$ and a power $p=0.78 \pm 0.02$, which flattens the adsorption well. All MD results presented below are for motion over this PES.

\subsubsection{Modelling diffusion on a lattice of fcc and hcp sites}

The process used to model the experimental results with MD simulations is an indirect one. Rather than using the experimental data as input parameters, simulations are run for a range of $\eta$ values and different shape and height potential energy surfaces, then treated in an identical way as the experimental data (cf. Section 5.4.1) to compare the $\alpha(\Delta \boldsymbol{K})$ curves and Arrhenius activation barriers to those obtained experimentally. The friction value and PES are altered to best represent the data. The interaction forces, $F_{i, j}$, are obtained from DFT simulations and included as $\alpha_{p}$ and $p_{0}$ (cf. Section 5.5). 

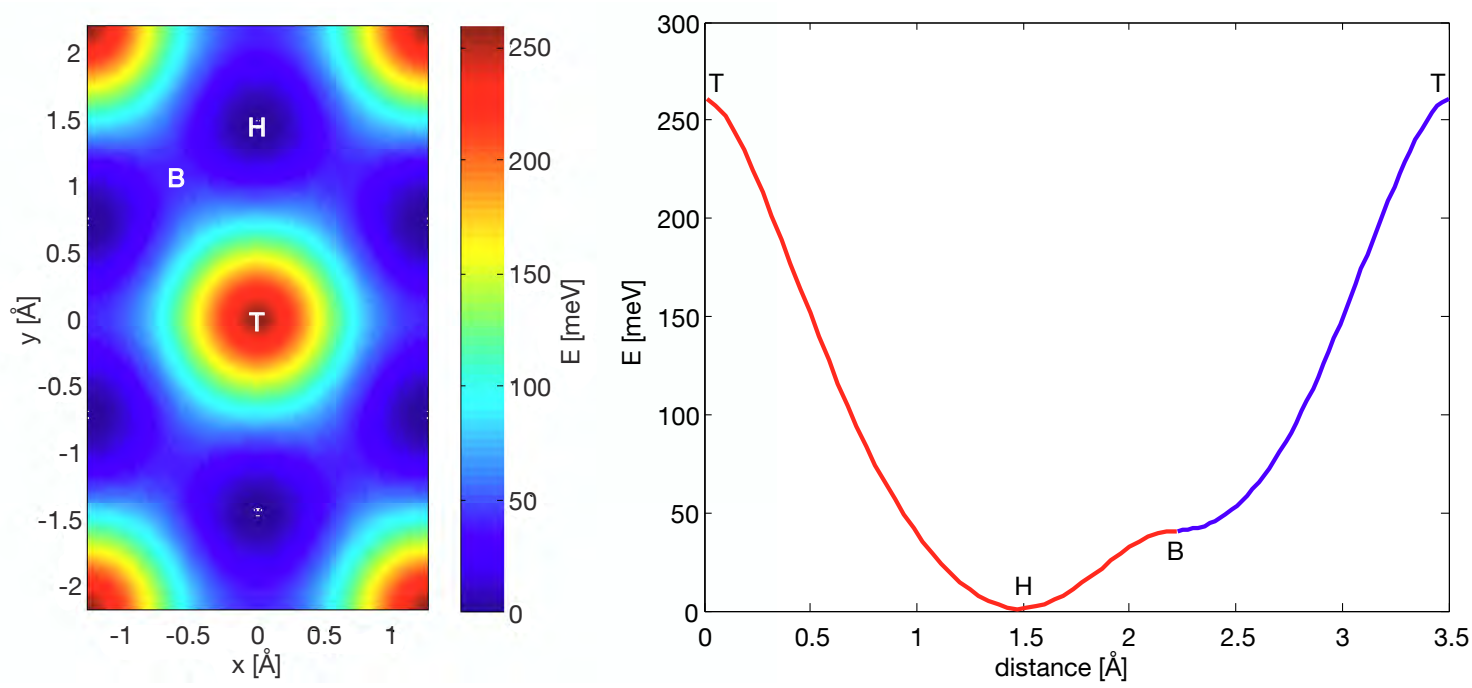

Figure 5.9: PES for hopping on a lattice of hollow sites used to simulate the $\mathrm{Cp} / \mathrm{Cu}(111)$ diffusion. The left-hand side panel shows a top view of the PES, while the righthand side panel represent a cross section from top to hollow and bridge site along $<11 \overline{2}>$ (red) and from bridge to top site along $<1 \overline{1} 0>$ (blue).

The shape and height of the PES influence the apparent Arrhenius activation energy as well as the jump rate. Since the maximum in the $\alpha(\Delta \boldsymbol{K})$ curve corresponds to the jump frequency, $\nu$, the PES also influences the magnitude of the dephasing rate, $\alpha$.

Friction, on the other hand, has an effect on the shape of the $\alpha(\Delta \boldsymbol{K})$ curve as well as its height. Altering the value for the friction coefficient while keeping all other parameters in the simulations identical illustrates this effect. A lower friction allows more double jumps and hence additional shorter sinusoids are added to the $\alpha(\Delta \boldsymbol{K})$ curve, resulting in a steeper rise at low $\Delta \boldsymbol{K}$ and a flatter peak, while increasing the friction moves further towards a single sinusoidal shape. Figure 5.10 shows the jump rate as a function of friction, deduced from MD simulations with $\eta$ as the only variable. As described in Section 2.4.3, Kramers predicted a deviation from transition state theory (TST) in the low and high friction regimes (cf. Figure 2.18) with a peak at the turnover region that is near the TST limit [45]. The predicted behaviour can be observed in the simulations presented in Figure 5.10.

Excellent agreement between simulations and $\mathrm{HeSE}$ data for $\mathrm{Cp} / \mathrm{Cu}(111)$ was obtained for the PES shown in Figure 5.9 and a friction coefficient, $\eta$, of $2.5 \pm 0.5 \mathrm{ps}^{-1}$ which is approximately on the peak of the curve for jump frequency as a function of friction (cf. Figure 5.10) and thus puts Cp diffusion near the TST limit. As expected for hopping on a lattice of degenerate fcc and hcp hollow sites, a single exponential lineshape is observed 


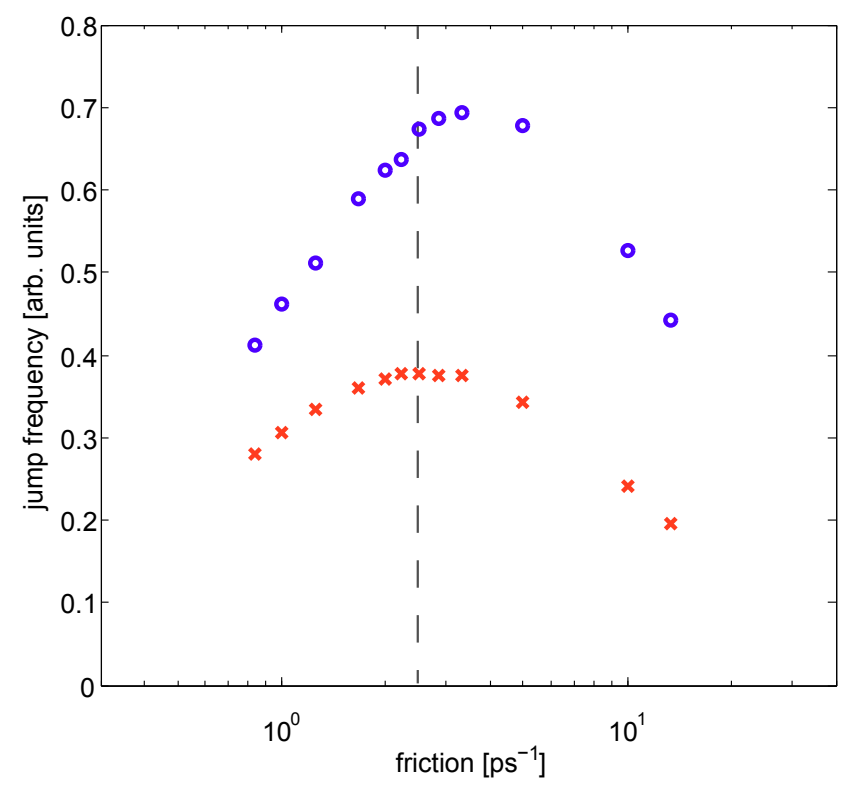

Figure 5.10: Jump frequency of $\mathrm{Cp} / \mathrm{Cu}(111)$ from $\mathrm{MD}$ simulations as a function of the friction coefficient. The jump frequency is obtained by determining the peak maximum in the $\alpha(\Delta \boldsymbol{K})$ curve. Red crosses are for the projection along $<11 \overline{2}>$ (using the slow decay), while blue circles are in the $<1 \overline{1} 0>$ direction. $\mathrm{Cp} / \mathrm{Cu}(111)$ exhibits a friction coefficient of $2.5 \mathrm{ps}^{-1}$ (grey dashed line) which is approximately on the peak of the curve and thus puts Cp diffusion close to the TST limit.

along $<1 \overline{1} 0>$, while double decays are found at certain momentum transfer values along $<11 \overline{2}>$. The simulated ISFs are analysed in the same way as the experimental data, using a double exponential function with the analytically calculated ratios of exponents and pre-factors to the curves, starting at $0.4 \mathrm{ps,} \mathrm{or} \mathrm{a} \mathrm{single} \mathrm{exponential} \mathrm{function} \mathrm{where}$ less than $1 \%$ intensity is predicted for the second decay.

From MD simulations at a range of different temperatures, an Arrhenius activation barrier of $E_{a}=42 \pm 1 \mathrm{meV}$ is obtained for data along both high symmetry crystal directions, in agreement with experimental values. It should be noted at this point, that the activation energy for diffusion obtained from Arrhenius measurements is not always equal to the energy barrier in the potential energy landscape [44]. The activation energy obtained from Arrhenius measurements is an "apparent" energy barrier and a function of the full form of the potential energy surface, and not just the height of the direct barrier to diffusion. Often, significant discrepancies between the experimental "apparent" barrier and the true barrier in the PES are found $[44,106]$. For MD simulations of $\mathrm{Cp} / \mathrm{Cu}(111)$, an Arrhenius activation energy of $42 \pm 1 \mathrm{meV}$ is accompanied by a diffusional barrier of $40 \pm 3 \mathrm{meV}$ in the potential energy landscape, a very close match of the two barriers. 
The $\alpha(\Delta \boldsymbol{K})$ curves obtained from the simulated ISFs at $300 \mathrm{~K}$ and $135 \mathrm{~K}$ are presented in Figures 5.11 and 5.12, respectively. Excellent agreement is found between simulations and experiment along the $<11 \overline{2}>$ direction at $300 \mathrm{~K}$. For the other azimuth at the same temperature, good agreement is found at $\Delta \boldsymbol{K}<2 \AA^{-1}$. However, the curve obtained from MD simulations is lower than the experimental values at larger momentum transfer values. At $135 \mathrm{~K}$ along $<11 \overline{2}>$ (cf. Figure 5.12), a deviation similar to that found comparing the experimental data to the analytical models is observed between experiment and MD simulation with decays systematically overestimated at high $\Delta \boldsymbol{K}$ in the simulation. Both deviations, along $<1 \overline{1} 0>$ at $300 \mathrm{~K}$ and along $<11 \overline{2}>$ at $135 \mathrm{~K}$, are due to the approximation of the energy difference between fcc and hcp sites as $\Delta E_{\text {hollow }}=0$, as confirmed in Section 5.8. These small deviations aside, the overall description of the data is very good and provides further information on the microscopic nature of $\mathrm{Cp}$ diffusion on $\mathrm{Cu}(111)$, such as the shape of the energy landscape (cf. Figure 5.9) and a friction coefficient.

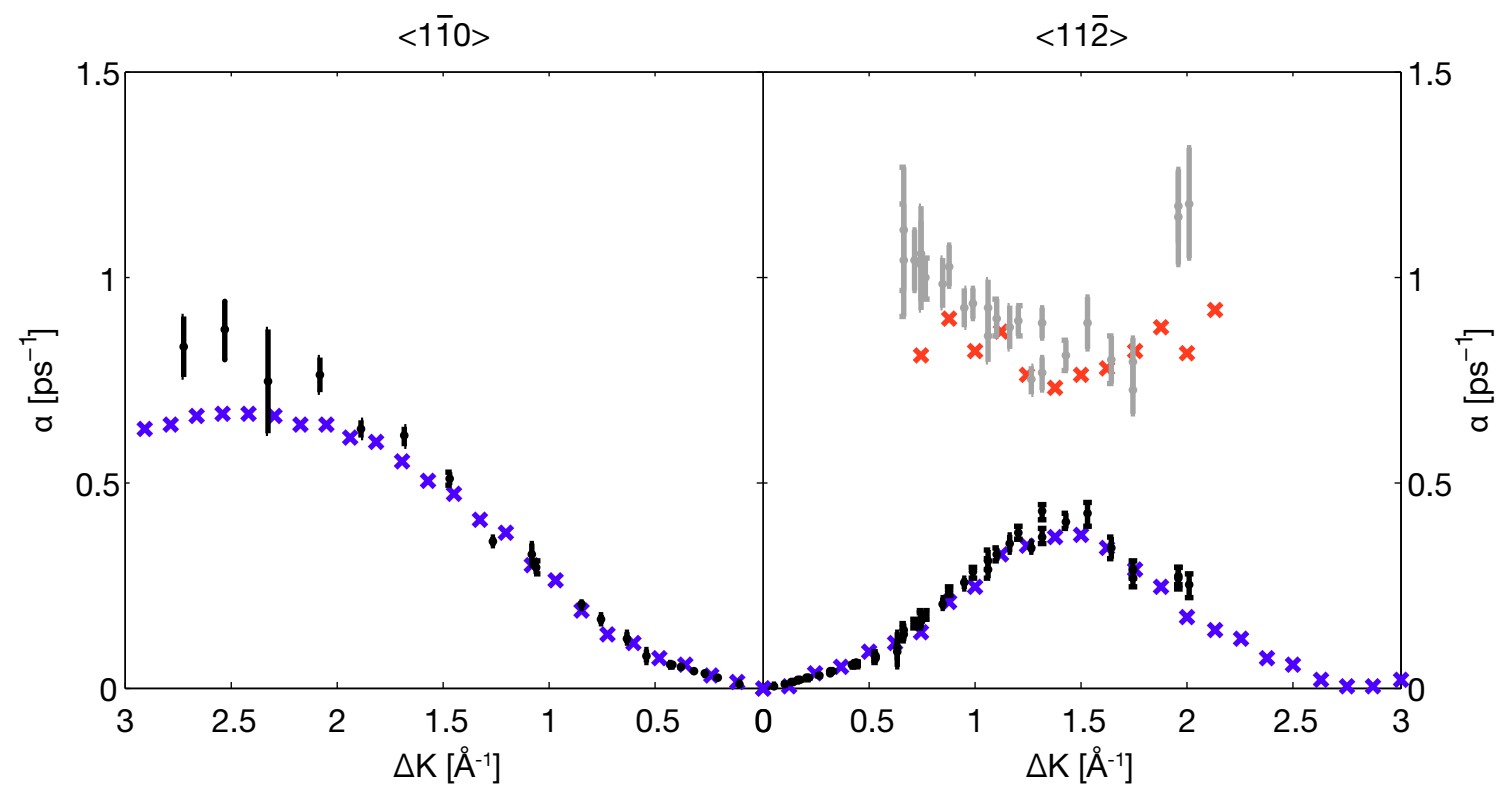

Figure 5.11: $\alpha(\Delta \boldsymbol{K})$ curves for $0.03 \mathrm{ML} \mathrm{Cp} / \mathrm{Cu}(111)$ at $300 \mathrm{~K}$. Points show experimental data and crosses the results from MD simulations for hopping on a non-Bravais lattice of degenerate hollow sites.

\subsubsection{Microscopic aspects of the friction}

The friction coefficient obtained for $\mathrm{Cp} / \mathrm{Cu}(111), \eta=2.5 \pm 0.5 \mathrm{ps}^{-1}$, is high compared to that of atomic adsorbate particles of similar mass. For example, on a $\mathrm{Cu}(100)$ surface 


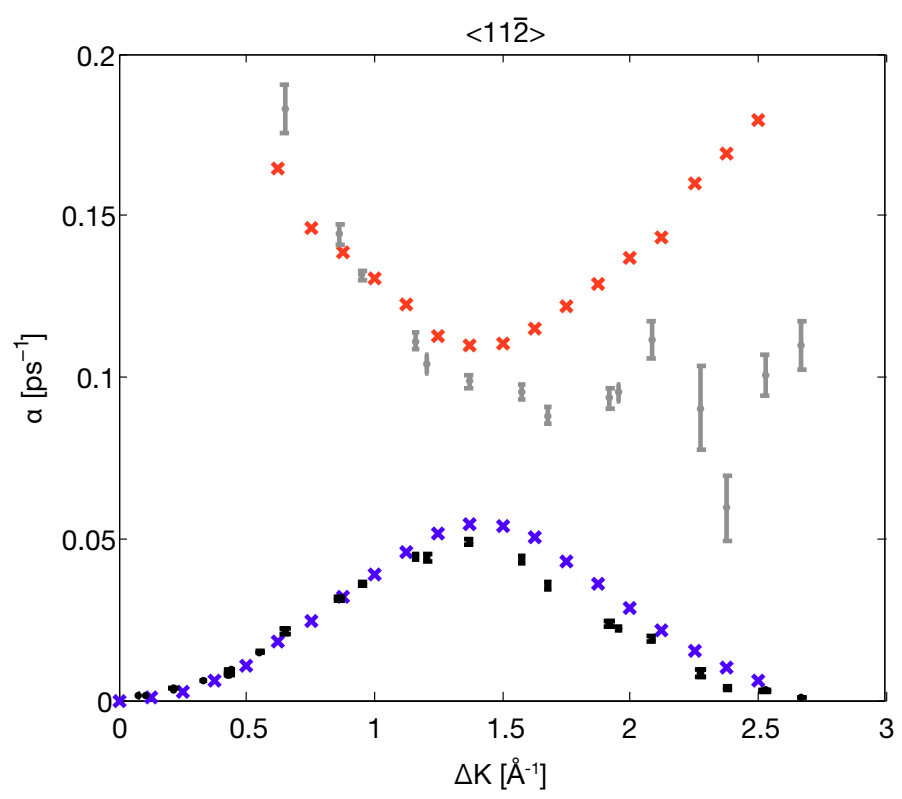

Figure 5.12: $\alpha(\Delta \boldsymbol{K})$ curves for $0.03 \mathrm{ML} \mathrm{Cp} / \mathrm{Cu}(111)$ at $135 \mathrm{~K}$. Points show experimental data and crosses the results from MD simulation for hopping on a non-Bravais lattice of degenerate hollow sites.

a friction of $\eta=0.5 \mathrm{ps}^{-1}$ was determined for $\mathrm{Na}$ [99], $\eta=0.22 \mathrm{ps}^{-1}$ for $\mathrm{K}$ [100], and $\eta=0.025 \mathrm{ps}^{-1}$ for Cs [31], while benzene/graphite has a much higher friction of $\eta=2.2 \mathrm{ps}^{-1}$ [8]. This implies that the extended nature of aromatic molecules increases the adsorbate-substrate energy transfer. In Chapters 6 and 7, similar behaviour is observed for two similar aromatic adsorbate molecules and a possible explanation for this phenomenon discussed.

Figure 5.13 shows typical trajectories of a single adsorbate during simulation. The adsorbate spends most of the time vibrating within an adsorption well, which is visible in the ISFs as the fast decay at short timescales. The determination of the friction coefficient from MD simulations confirms that the intracell diffusion signatures appear below $1 /\left(2.5 \mathrm{ps}^{-1}\right)=0.4 \mathrm{ps}$ and the lineshapes beyond 0.4 ps thus describe intra-cell diffusion.

Furthermore, the trajectories primarily show hopping between nearest neighbour hollow sites and only a relatively small number of double jumps. This qualitative observation can be quantified further by estimating the probability of a single jump as proportional to $\exp (t \eta)$, and the probability of a double jumps proportional to exp $(2 t \eta)$. 


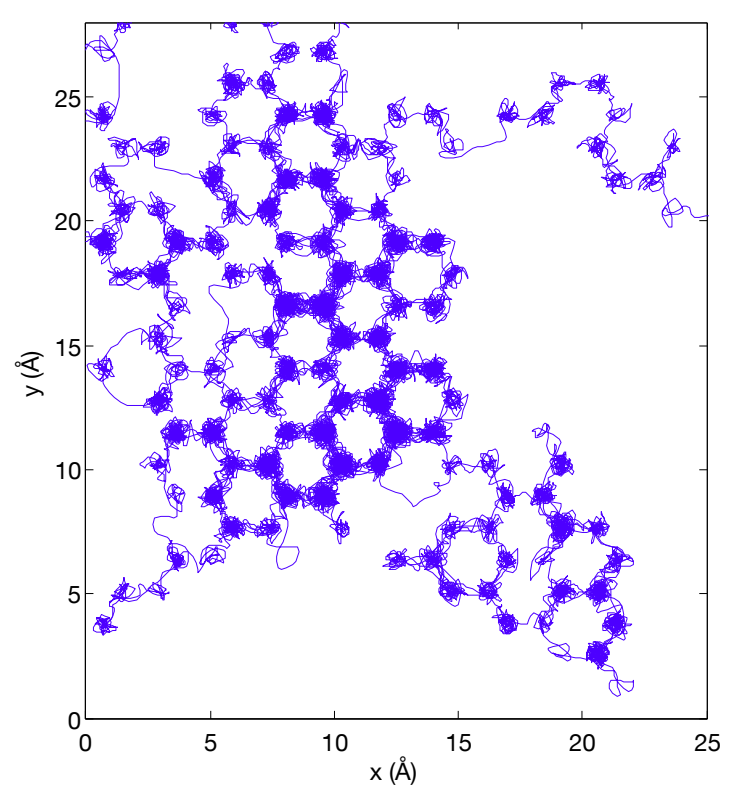

Figure 5.13: Trajectories for $\mathrm{MD}$ simulations of $\mathrm{Cp} / \mathrm{Cu}(111)$ show hopping on a non-Bravais lattice of energetically degenerate hollow sites.

The relative proportion of double jumps is hence given as

$$
\frac{\exp (2 t \eta)}{\exp (t \eta)+\exp (2 t \eta)}
$$

The time for a jump, $t$, can be estimated from $t=d / v=d / \sqrt{2 k_{B} T / m}$, where $d$ is the distance between two adjacent hollow sites, $v$ the particle velocity, $k_{B}$ the Boltzmann constant, $T$ the sample temperature, and $m$ the adsorbate mass. Using the friction coefficient from MD simulations, a proportion of $21 \%$ double jumps is found for $\mathrm{Cp} / \mathrm{Cu}(111)$ motion at $300 \mathrm{~K}$. The $\alpha(\Delta \boldsymbol{K})$ curve is therefore dominated by single jumps, explaining the good agreement between experiment and analytical model which does not include double jumps (cf. Figure 5.6).

To conclude, classical Langevin MD simulations have been applied successfully, modelling the diffusion of $\mathrm{Cp}$ on a lattice of fcc and hcp sites on $\mathrm{Cu}(111)$. Excellent agreement with the experiment is obtained in the momentum transfer and temperature dependence of the diffusion. A friction coefficient of $2.5 \pm 0.5 \mathrm{ps}^{-1}$ was determined, corresponding to $21 \%$ double jump contribution. 


\subsection{Weak lateral interactions}

As noted earlier, no clear de Gennes feature is apparent in the $\alpha(\Delta \boldsymbol{K})$ curves for $\mathrm{Cp} / \mathrm{Cu}(111)$. Therefore, one would not expect the work function and dipole moment obtained from DFT calculations to create dips in the curves from MD simulations either. Indeed, the results shown in Figures 5.11 and 5.12 are for simulations including dipolar interactions as defined by first principles calculations and show good agreement with experiment.

A closer look at the low momentum transfer region, where lateral interactions would be apparent, however, reveals a small deviation from the analytical model curves, as shown in Figure 5.14. The experimental curves (blue dots) rise above the predicted line (grey) at small $\Delta \boldsymbol{K}$ values, then briefly fall below before rejoining the analytical line. The same behaviour is observed in the curves from MD simulations (red crosses). This small deviation from a simple sinusoid can be interpreted as a very weak de Gennes feature, implying that $\mathrm{Cp}$ adsorbates do indeed repel each other, if only weakly so. Figure 5.14 also suggests that the dipole moment from DFT calculations is in agreement with the experiment as simulations including interactions describe the experimental data well.

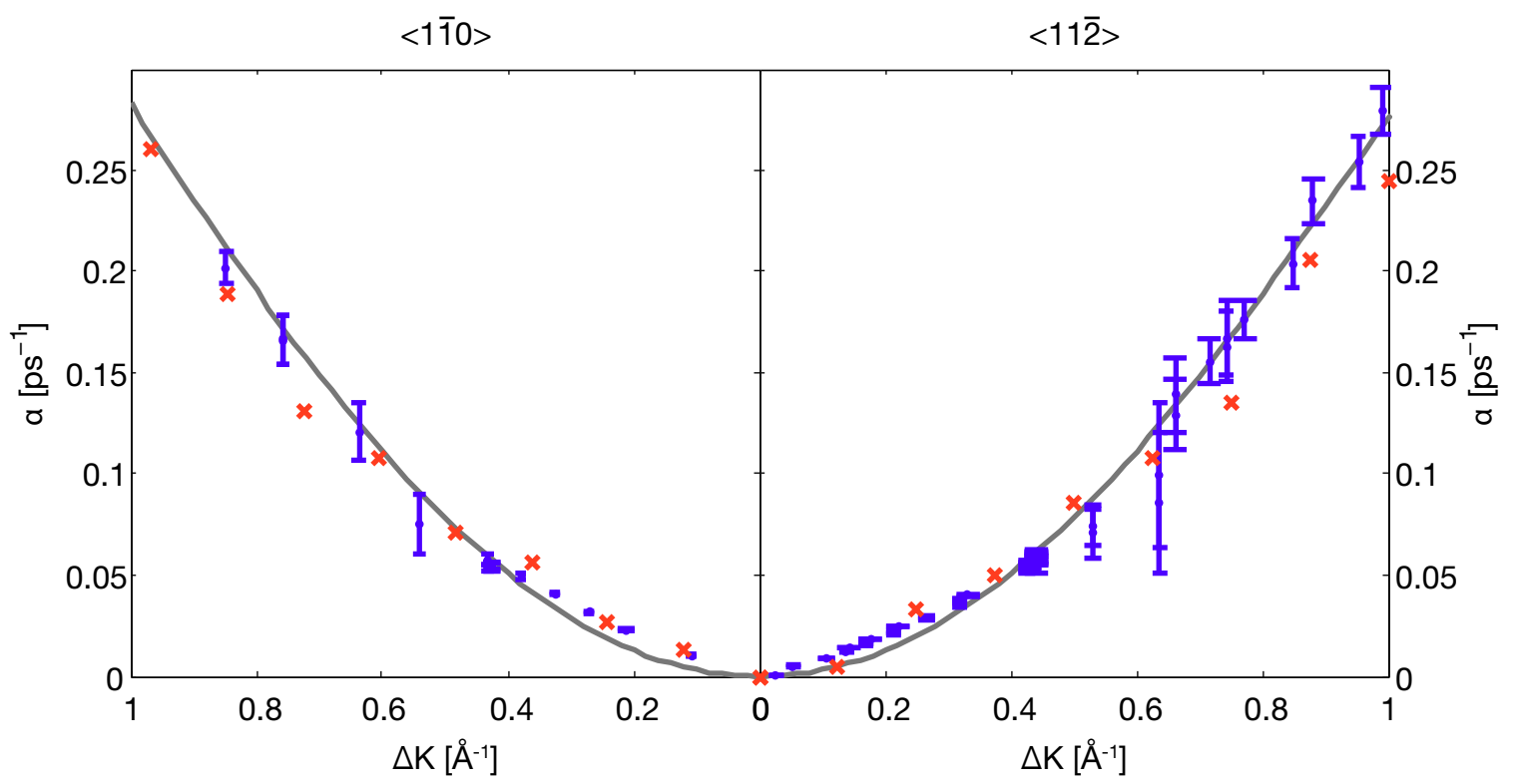

Figure 5.14: $\alpha(\Delta \boldsymbol{K})$ curves for $\mathrm{Cp} / \mathrm{Cu}(111)$ at $300 \mathrm{~K}$ showing a weak de Gennes feature at low $\Delta \boldsymbol{K}$ when compared with the analytical model (grey lines). Results from MD simulations are shown as red crosses, experimental data are plotted as blue dots. 
In addition, signatures from repulsive lateral interactions provide a means of estimating the adsorbate coverage using Equation 2.24. In the experimental data, the dip position is at approximately $0.5 \AA^{-1}$, corresponding to a coverage of $0.03 \mathrm{ML}$, thus confirming the coverage estimate presented in Section 5.3 using a cross section of $200 \AA^{2}$. Furthermore, a dip position of $0.5 \AA^{-1}$ does not correspond to any of the peaks in the diffraction scans shown in Figure 5.3, implying that the features observed in diffraction measurements are form factor rings.

The data presented in Figure 5.14 demonstrate that HeSE is uniquely sensitive to even the weakest of repulsive inter-adsorbate interactions and provides a means of verifying the interaction forces calculated by DFT.

\subsection{Determining the energy difference between fcc and hcp sites}

According to the analytical model for hopping on a lattice of fcc and hcp sites, presented in Section 2.4.1, an energy difference between the two hollow sites results in a change in the ratio of the two decays along $<11 \overline{2}>$ and the appearance of a second decay along $<1 \overline{1} 0>$, growing in intensity as the energy difference increases [38]. The ISF at a particular $\Delta \boldsymbol{K}$ value is given by Equation 2.21, which is dependent on two variables: the residence time, $\tau$ - which is the inverse of the jump attempt frequency, $\nu$ - and a parameter $\lambda-$ the ratio between the jump rates from fcc to hcp and from hcp to fcc sites. The jump rate is defined by

$$
\frac{1}{\tau}=\Gamma_{0} \cdot \exp \left(-\frac{E}{k_{B} T}\right)
$$

and hence $\lambda$ can be written as

$$
\lambda=\frac{\Gamma_{0} \cdot \exp \left(-\frac{E_{1}}{k_{B} T}\right)}{\Gamma_{0} \cdot \exp \left(-\frac{E_{2}}{k_{B} T}\right)}=\exp \left(\frac{\Delta E_{\text {hollow }}}{k_{B} T}\right),
$$

where $\Delta E_{\text {hollow }}$ is the energy difference between the two kinds of hollow sites, $k_{B}$ the Boltzmann constant, $T$ the temperature and $\Gamma_{0}$ a prefactor related to the vibrational frequency at the adsorption site and the friction [38]. Rearranging Equation 5.10 gives 
the energy difference as

$$
\Delta E_{\text {hollow }}=k_{B} T \cdot \ln \lambda
$$

HeSE experiments are typically analysed by investigating each ISF individually [8,44], which is the method employed above. From the lineshape in individual HeSE measurements, it could be estimated that the energy difference between fcc and hcp sites, $\Delta E_{\text {hollow }}$, is small as there is no evidence of double decays along $<1 \overline{1} 0>$. The noise level in an individual ISF, however, is too large to allow a more accurate determination of the energy difference as a small contribution of a second decay could be hidden. In order to obtain a global picture of the dynamics, allowing an accurate determination of $\Delta E_{\text {hollow }}$, a more complete, rigorous method of analysing all ISFs together is employed. Bayesian statistics provides a means of such a global analysis of all spectra [108]. Rather than describing each single ISF separately, a Bayesian method searches for the probability that a model describes all data correctly. The probability is calculated for a range of models and normalised so that the overall probability of the sum of all models is equal to one.

Bayes' theorem

$$
P(M \mid D)=\frac{P(D \mid M) P(M)}{P(D)}
$$

states that the probability that a proposed model $M$ is right given the data $D$, is given by the product of the probability that the data $D$ is represented by model $M$ and the probability that the model itself is correct, divided by the probability of the data $D$ [108]. The total probability,

$$
P(D \mid M)=\prod_{i} P\left(d_{i} \mid m_{i}\right)
$$

is the product of the probability of each individual $\mathrm{ISF}^{1}$. A more detailed description of the method employed here can be found in reference [27].

In the case of the diffusion of $\mathrm{Cp} / \mathrm{Cu}(111)$, the model is a sum of two exponential functions, $a_{1} \cdot \exp \left(-\alpha_{1} t\right)+a_{2} \cdot \exp \left(-\alpha_{2} t\right)+c$, where the prefactors and exponents are defined by Equation 2.21 for a particular pair of $\lambda$ and $\tau$ values, the only variables in the model. To analyse the potential energy landscape observed by a diffusing $\mathrm{Cp}$ on a

\footnotetext{
${ }^{1}$ To simplify the calculation, the sum of the logarithm of the probability is taken instead of multiplying
} the probabilities. 
$\mathrm{Cu}(111)$ substrate, the probability for each combination of $\lambda$ and $\tau$ values in a selected $\lambda-\tau$ space is calculated, giving the probability distribution as a function of $\lambda$ and $\tau$ for each measured ISF.

For ISFs which have little contribution from a second decay, a rather flat probability distribution is found, implying that no single model is particularly favourable over the others. Therefore, only ISFs which show a clear peak in the probability distribution have been included in the statistical analysis, namely at $\Delta \boldsymbol{K}>1.0 \AA^{-1}$ along the $<11 \overline{2}>$ azimuth. As observed qualitatively before, no clear double decay is visible along $<1 \overline{1} 0>$ and thus the probability distribution is flat for all data in this direction. A probability distribution in $\lambda-\tau$ space is obtained for each HeSE measurement, and the total probability that the data from all relevant ISFs are consistent with particular values of $\lambda$ and $\tau$ space is calculated as per Equation 5.13. Since the temperature appears in

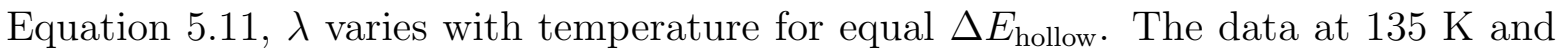
$300 \mathrm{~K}$ have thus been treated separately. Figure 5.15 (A) and (C) shows the probability distribution obtained at $135 \mathrm{~K}$ and $300 \mathrm{~K}$, respectively. Maximum probability is found for $\lambda=1.73$ and $\tau=1.16$ at $300 \mathrm{~K}$, and $\lambda=2.86$ and $\tau=5.86$ at $135 \mathrm{~K}$.

The results can be marginalised over $\tau$, by integrating the probability over $\tau$ for a particular value of $\lambda$, to give the probability as a function of $\lambda$ for each data set (cf. panels B and D in Figure 5.15) [108]. The optimum $\lambda$ values are in agreement with the non-marginalised solution, and the peak width provides a standard deviation. Using Equation 5.11, an energy difference between fcc and hcp hollow sites of $\Delta E_{\text {hollow }}=$ $12.2 \pm 0.3 \mathrm{meV}$ at $135 \mathrm{~K}$ and $\Delta E_{\text {hollow }}=14.2 \pm 2.0 \mathrm{meV}$ at $300 \mathrm{~K}$ is found. Combining the results at both temperatures by multiplying the Gaussian distributions gives an overall energy difference between fcc and hcp sites of $\Delta E_{\text {hollow }}=12.3 \pm 0.3 \mathrm{meV}$. As originally estimated from the shape of individual ISFs, this energy difference is fairly small compared to the barrier to diffusion $(41 \pm 1 \mathrm{meV})$, yet non-negligible.

The non-Bravais model for jumps between adjacent fcc and hcp sites with an energy difference of $12.3 \mathrm{meV}$ predicts slightly different ratios between prefactors $a_{1}$ and $a_{2}$ as well as exponents $\alpha_{1}$ and $\alpha_{2}$ in Equation 5.1 than the model for degenerate hollow sites used in Section 5.4.2. In addition, the lineshapes in the $<1 \overline{1} 0>$ direction change from a single exponential $\left(a_{2}=0\right.$ in Equation 5.1) in the degenerate case to a double decay $\left(a_{2} \neq 0\right)$ and thus have to be analysed in the same way as the data along the other azimuth. Figures 5.16 and 5.17 present the data described by the improved model at $300 \mathrm{~K}$ and $135 \mathrm{~K}$, respectively, showing excellent agreement with the analytical model. 

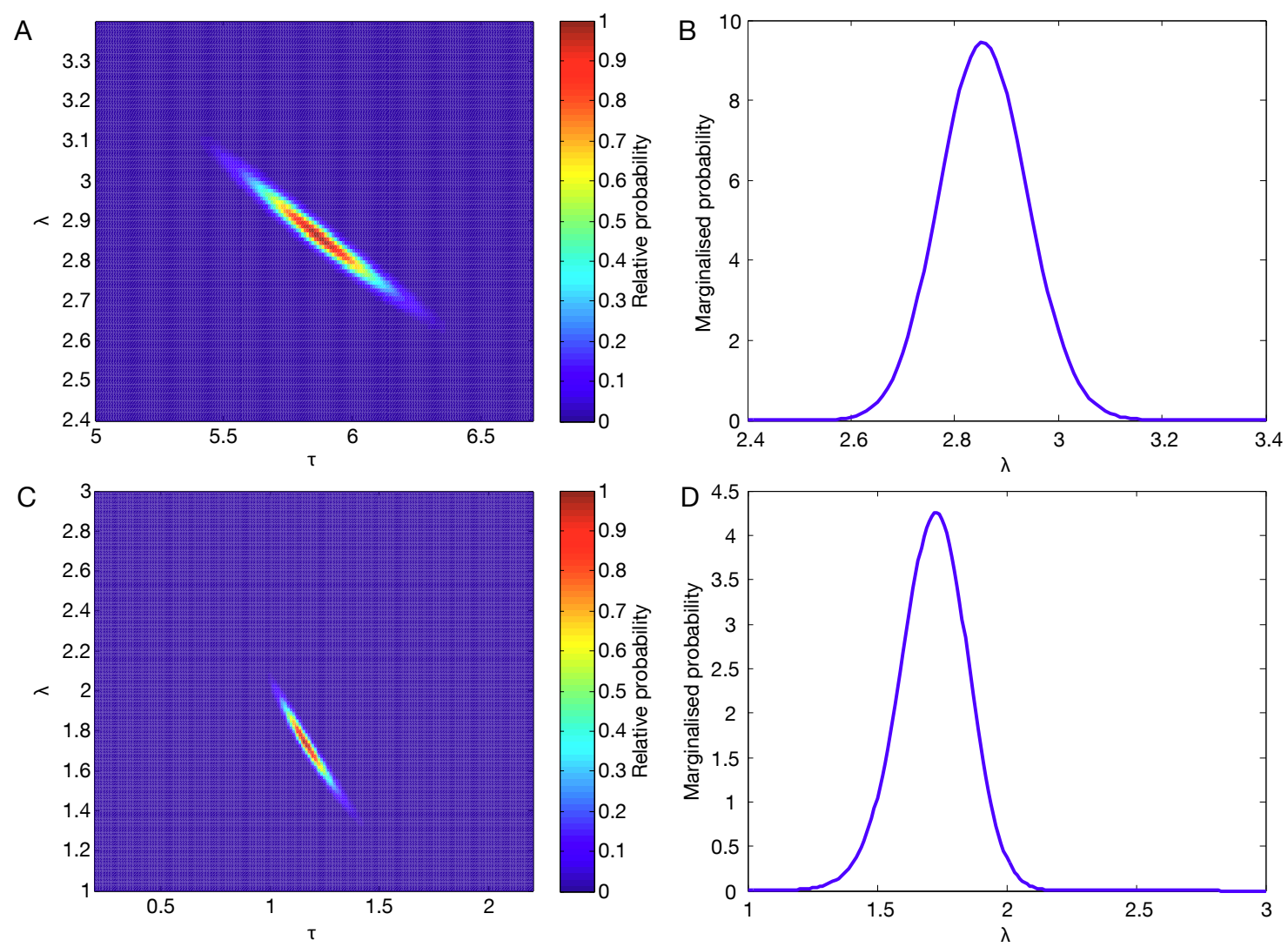

Figure 5.15: Relative probability of analytical models for jump diffusion between fcc and hcp sites for $\mathrm{Cp} / \mathrm{Cu}(111)$. (A) and (C) illustrate the probability distribution in $\lambda-\tau$ space for $\mathrm{Cp} / \mathrm{Cu}(111)$ at $135 \mathrm{~K}$ and $300 \mathrm{~K}$, respectively, showing a clear peak (red). After marginalisation over all values in $\tau$, the probability as a function of $\lambda$ is obtained for Cp diffusion at (B) $300 \mathrm{~K}$ and (D) $135 \mathrm{~K}$.

At $300 \mathrm{~K}$ along $<11 \overline{2}>$ (cf. Figure 5.16), the agreement between experiment and model is comparable to that for degenerate hollow sites (cf. Figure 5.6). The gap between slow and fast decay is increased as defined by the analytical model. A second decay becomes apparent along the $<1 \overline{1} 0>$ direction (cf. Figure 5.16), splitting open a gap where the two predicted lines in the degenerate model cross (cf. Figure 5.6). Up to around $1.6 \AA^{-1}$, the slow decay dominates (i.e. has more intensity than the fast decay), while the fast decay is more important at larger momentum transfers. The curves of the respective dominant processes together resemble the original curve shown in Figure 5.6. The weak contribution of a second decay at large momentum transfer values in this crystal direction is the most likely cause for the small deviation of MD simulations for degenerate hollow sites from the experiment in this region. 


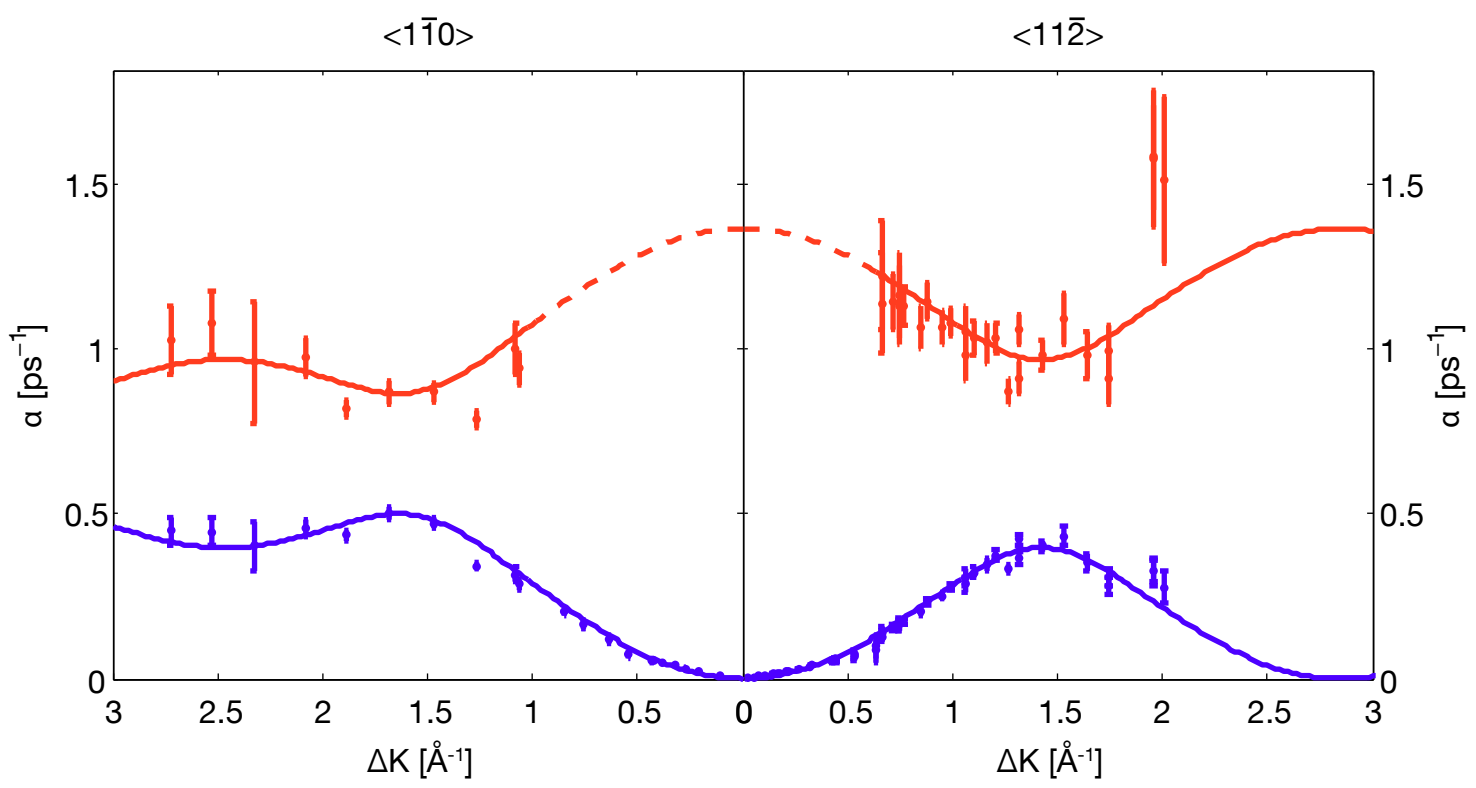

Figure 5.16: $\alpha(\Delta \boldsymbol{K})$ curves for $0.03 \mathrm{ML} \mathrm{Cp} / \mathrm{Cu}(111)$ at $300 \mathrm{~K}$, describing the data by a model for hopping between fcc and hcp hollow sites with an energy difference of $12.3 \mathrm{meV}$. Two decays are now visible along both azimuths. The solid lines show the analytical model for single jumps between hollow sites, while the dashed lines illustrate predicted decays with less than $1 \%$ intensity.

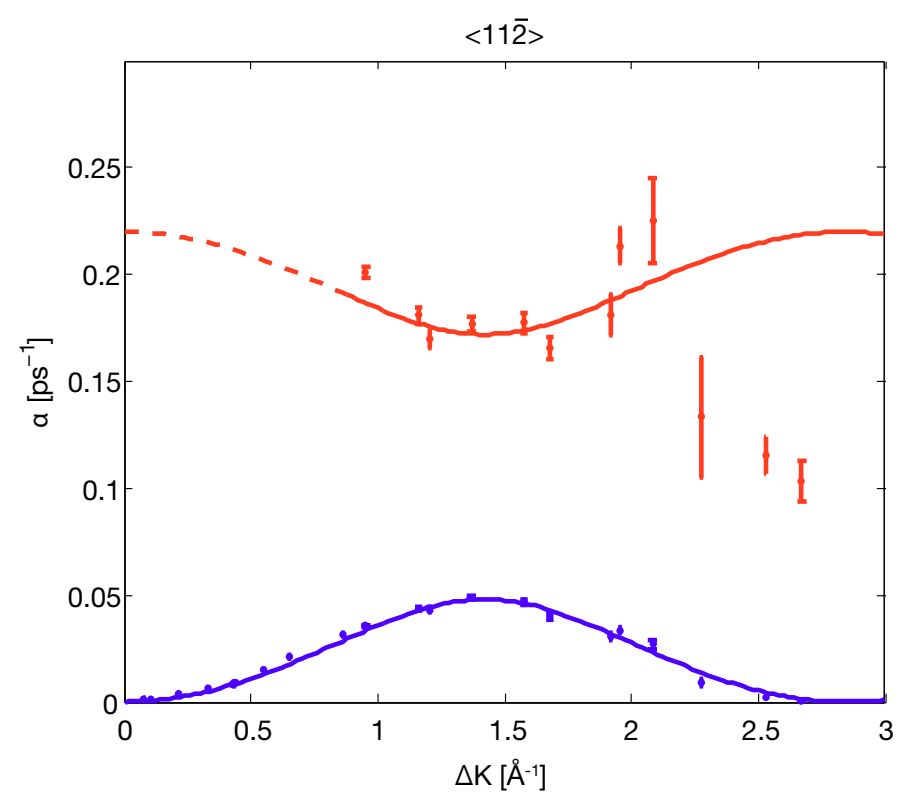

Figure 5.17: $\alpha(\Delta \boldsymbol{K})$ curves for $0.03 \mathrm{ML} \mathrm{Cp} / \mathrm{Cu}(111)$ at $135 \mathrm{~K}$, describing the data by a model for hopping between fcc and hcp hollow sites with an energy difference of $12.3 \mathrm{meV}$. The solid lines show the analytical model for single jumps between hollow sites, while the dashed lines illustrate predicted decays with less than $1 \%$ intensity. 
At $135 \mathrm{~K}$, the new model represents a much improved representation of the data (cf. Figure 5.17) than that for degenerate hollow sites (cf. Figure 5.7). In particular in the centre region of the $\Delta \boldsymbol{K}$ scale, the decays follow the predicted line, and the asymmetry of the decay curves observed in Figure 5.7 has disappeared by including $\Delta E_{\text {hollow }}=12.3 \pm 0.3 \mathrm{meV}$.

In order to investigate the diffusion of $\mathrm{Cp} / \mathrm{Cu}(111)$ on an energy landscape with $\Delta E_{\text {hollow }}=12.3 \pm 0.3 \mathrm{meV}$, MD simulations on a PES reflecting this geometry would be required. To optimise the PES and friction value, the results of each MD simulation would need to be analysed using the Bayesian method applied to the experiment, making it a computationally intensive and tedious, if not impossible, procedure. Fortunately, $\Delta E_{\text {hollow }}$ is sufficiently small that the simulations presented earlier are a valid approximation and the friction coefficient is not expected to be influenced significantly.

\subsection{Intracell diffusion as a handle on friction and spring constant}

In addition to jump diffusion, the HeSE data show evidence of intracell diffusion of $\mathrm{Cp} / \mathrm{Cu}(111)$, i.e. the dephasing of vibrations within the adsorption well in between the individual hops, which typically occurs on faster timescales than jump diffusion. Analysis of the fast decay provides a first experimental investigation of intracell diffusion. The momentum transfer dependence of the dephasing rate of intracell diffusion was previously predicted using MD simulations [98]. By describing the lineshape with an exponential (or, in the energy regime, a Lorentzian), Jardine et al. found a constant energy broadening, or equivalently dephasing rate, at small momentum transfers that corresponds to the friction coefficient, $\eta$, and increasing values at larger $\Delta \boldsymbol{K}_{\mathrm{s}}$ [98]. Their results are reproduced in the right-hand side panel of Figure 5.18, where the "fitted width" corresponds to the energy broadening or dephasing rate. The transition from constant to rising $\alpha$ occurs roughly when the scattering phase reaches $\Phi=\Delta \boldsymbol{K} \cdot \boldsymbol{R}=\pi / 4$, where $\boldsymbol{R}$ is the mean displacement of the adsorbate within an adsorption well.

It should be noted that the present data are not ideally suited for the study of intracell diffusion, due to fairly coarse point spacing at small spin-echo times and because it was found retrospectively that the two point measurement technique applied during these experiments is prone to experimental errors at short timescales, where the signature for 

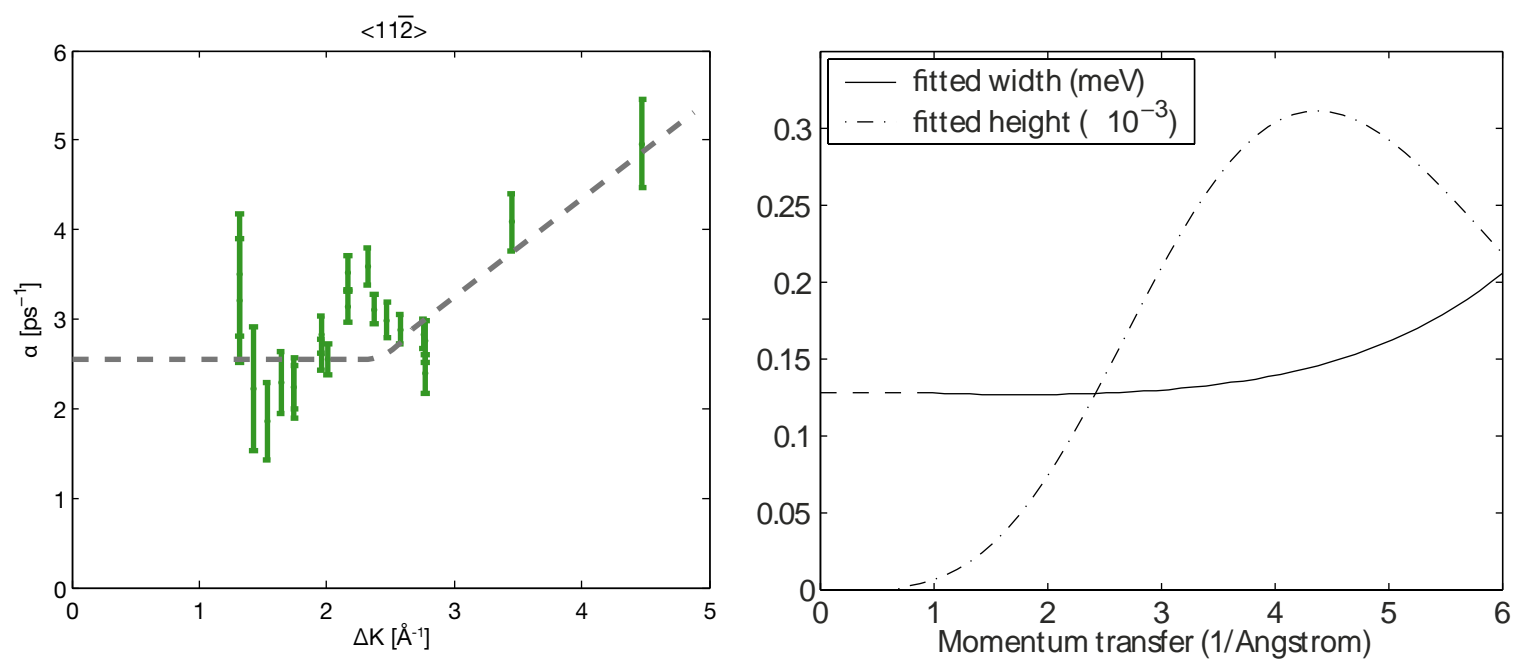

Figure 5.18: The panel on the left shows the $\alpha(\Delta \boldsymbol{K})$ curve for intracell diffusion of $\mathrm{Cp} / \mathrm{Cu}(111)$ at $300 \mathrm{~K}$. The dashed line illustrates schematically the shape predicted in [98], which is reproduced in the panel on the right.

intracell diffusion is found (cf. Section 2.3). However, while the precise results must be treated with caution, the general findings provide an important addition to the existing theoretical framework $[98,109]$, and demonstrate that $\mathrm{Cp} / \mathrm{Cu}(111)$ is an adsorbate system uniquely suited for the investigation of motion within an adsorption well.

For a flat energy landscape, it has been shown theoretically that the lineshapes in ISFs are of a very complex nature [33,109], and can consist of a sum of exponential and Gaussian functions. At long times, the exponential shape dominates, while the Gaussian shape becomes more important at very fast timescales. Except for the numerical simulations in [98], little is known about the expected lineshape in the case of intracell motion. An approximate analytical solution to the problem of a harmonic oscillator and a heat bath [110] suggests the vibrational correlations should decay exponentially with time. For this reason, the experimental data were described by an exponential function, extending Equation 5.1 to include a third exponent for the fast decay: $f(t)=$ $a_{1} \cdot \exp \left(-\alpha_{1} t\right)+a_{2} \cdot \exp \left(-\alpha_{2} t\right)+a_{3} \cdot \exp \left(-\alpha_{3} t\right)+c$. The ratios $a_{1} / a_{2}$ and $\alpha_{1} / \alpha_{2}$ are defined by the model for diffusion on fcc and hcp hollow sites as described above and agree with the ones presented in Figure 5.16. Figure 5.18 shows the $\alpha(\Delta \boldsymbol{K})$ curve for the intracell diffusion of $\mathrm{Cp} / \mathrm{Cu}(111)$ along $<11 \overline{2}>$ at $300 \mathrm{~K}$. At $\Delta \boldsymbol{K}<1.2 \AA^{-1}$, intracell diffusion has little intensity, making it difficult to extract any information. Therefore, only data at $\Delta \boldsymbol{K}>1.2 \AA^{-1}$ are used. 
The overall shape of the curve for intracell diffusion agrees well with the prediction from numerical simulations in [98] (compare the data with the grey dashed line drawn on the data to guide the eye). Figure 5.18 shows that the effective dephasing rate for the fast component extrapolates, as $\Delta \boldsymbol{K} \rightarrow 0$, to a value that is consistent with the friction coefficient determined from MD simulations $\left(2.5 \pm 0.5 \mathrm{ps}^{-1}\right)$, in agreement with [98]. Extrapolating the flat end of the curve therefore provides a handle on the friction coefficient, $\eta$, independent of the standard method using MD simulations to model the intra-cell diffusion. At higher $\Delta \boldsymbol{K}$, the fast decay increases approximately linearly with $\Delta \boldsymbol{K}$. Such behaviour is expected of ballistic motion [111], suggesting that the fast component at high $\Delta \boldsymbol{K}$ is a measure of the ballistic behaviour of the molecule.

The take-off point where the curve changes from constant to rising $\alpha$ is at approximately $\Delta \boldsymbol{K}=2.5 \pm 0.4 \AA^{-1}$, indicating that the mean displacement of Cp within the adsorption well is $\boldsymbol{R}=0.31 \pm 0.05 \AA$. This result can be used to estimate the spring constant, $k$, for $\mathrm{Cp}$ vibrations through the relationship

$$
E=\frac{1}{2} k_{B} T=\frac{1}{2} k \boldsymbol{R}^{2}
$$

where $k_{B}$ is the Boltzmann constant and $T$ the sample temperature (here $300 \mathrm{~K}$ ). With the mean displacement estimated from the take-off point of the intracell diffusion curve, a spring constant of $k=4.2 \pm 1.4 \mathrm{~N} / \mathrm{m}$ is obtained. To get an idea of the quality of the result, a second, independent method to estimate the spring constant can be applied. From the curvature of the adsorption well in the PES shown on the right-hand side in Figure 5.9, a spring constant of $k=5.3 \pm 0.1 \mathrm{~N} / \mathrm{m}$ is found as a more reliable value, in good agreement with the estimate from the intracell diffusion curve.

From the spring constant, the energy of the Cp vibrations can be estimated through

$$
E=\hbar \omega=\hbar \sqrt{\frac{k}{m}},
$$

where $\hbar$ is the reduced Planck constant, $\omega$ the angular frequency and $m$ the mass of Cp. For $k=5.3 \pm 0.1 \mathrm{~N} / \mathrm{m}$, a predicted energy of $4.6 \pm 0.1 \mathrm{meV}$ is obtained, in excellent agreement with the energy (4.6 meV) of the non-dispersive mode of $\mathrm{Cp} / \mathrm{Cu}(111)$ observed in Figure 5.5. The $4.6 \mathrm{meV}$ mode can therefore be attributed to a translational mode (T-mode) of Cp. Furthermore, in the dispersive mode shown as blue crosses in Figure 5.5, a kink is apparent at approximately $4.6 \mathrm{meV}$, indicating resonances of the substrate phonon modes with the T-mode of Cp. 
The method of estimating the friction value from intracell motion was employed previously for the interpretation of the motion of propane/Pt(111) [112], however the assumption had to be made that the simulations presented in [98] provided sufficient evidence to justify the approach. The analysis of $\mathrm{Cp}$ intracell motion is the first time that the simulations by Jardine et al. [98] could be verified experimentally. Chapter 7 illustrates that the standard method of using MD simulations to get a handle on the friction cannot always be employed, in which case the intracell diffusion method would be a useful alternative approach.

\subsection{Conclusions}

In this chapter, HeSE measurements investigating the surface diffusion of the fivemembered aromatic adsorbate species, $\mathrm{Cp}$, on the hexagonal $\mathrm{Cu}(111)$ surface have been presented. Cp was found to move predominantly in single hops between adjacent fcc and hcp hollow sites, over an apparent activation barrier of $41 \pm 1 \mathrm{meV}$. On an fcc(111) surface, both kinds of hollow sites together form a non-Bravais lattice, thus giving rise to multi-component lineshapes in the ISFs. Although these signatures have been predicted previously [38], the data presented here provide first experimental evidence of clear double decays due to hopping on a non-Bravais lattice [101].

In addition, exceptionally weak lateral interactions are observed. DFT calculations performed by Sacchi and Jenkins [101,102] explain the lack of repulsive interactions in this ionically bonded adsorbate species. Electron depletion above the topmost copper substrate atoms upon adsorption of $\mathrm{Cp}$ creates a dipole moment in addition to a dipole moment in the opposite direction, induced by the charge transfer of 1.1 electrons from the surface to the Cp. This so-called "cushion effect" results in near cancellation of two dipoles and thus a surprisingly weak total dipole moment.

MD simulations confirm hopping motion on a lattice of nearly degenerate hollow sites as well as the dipole moment from DFT calculations and provide a friction coefficient of $2.5 \pm 0.5 \mathrm{ps}^{-1}$. The friction is strong compared to that of atomic adsorbate species of similar mass and dipole moment, suggesting that the extended nature of $\mathrm{Cp}$ increases the adsorbate-substrate energy transfer.

In addition, a Bayesian analysis method was performed to determine the energy difference between fcc and hcp sites accurately. Probing the $\lambda-\tau$ parameter space, i.e. 
the two variables in the non-Bravais jump model, $\Delta E_{\text {hollow }}=12.3 \pm 0.3 \mathrm{meV}$ has been determined. Excellent agreement between experimental data and the analytical model for this energy difference was found, both at low and at high temperatures.

Finally, the intracell motion of $\mathrm{Cp} / \mathrm{Cu}(111)$ was investigated, providing a first experimental confirmation of the signatures predicted by simulations in [98]. Intracell motion gives a handle on the friction and spring constant for $\mathrm{Cp} / \mathrm{Cu}(111)$, in good agreement with the values from MD simulations of the jump diffusion. 


\section{Chapter 6}

\section{Quantum influences in the diffusive motion of pyrrole on $\mathrm{Cu}(111)$}

Following the study of the dynamics of cyclopentadienyl (Cp) on $\mathrm{Cu}(111)$ in the previous chapter, this chapter investigates the motion of an analogous heteroaromatic adsorbate on the same surface, pyrrole/ $\mathrm{Cu}(111)$.

Pyrrole is an unsaturated organic five-membered ring compound with the formula $\mathrm{C}_{4} \mathrm{H}_{4} \mathrm{NH}$. The $\pi$-electrons of the four carbon atoms together with the lone pair on the nitrogen atom form a stable aromatic ring, similar to that of benzene. Compared to $\mathrm{Cp}$, one of the carbon atoms is replaced with a nitrogen atom, thus making it a stable, neutral molecule in the gas phase.

Like Cp, pyrrole is an important precursor for many technologically relevant materials; for example, pyrrole derivatives are found in organometallic complexes [113]. In recent years, pyrrole and pyrrole-related molecules have attracted increasing attention as precursor molecules for self-assembled monolayers (SAM) on metal surfaces [114,115]. During the process of self-assembly, the adsorbates must be mobile in order to align themselves with respect to each other and form regular structures.

Pyrrole is also one of the building blocks of biochemical macrocycles such as porphyrins (e.g. haem), chlorins (e.g. chlorophyll) and corrins (e.g. vitamin $\mathrm{B}_{12}$ ). In addition, pyrrole can be electrochemically polymerised to form polypyrrole, a chain of 2,5-connected pyrrole molecules. This polymer has interesting applications in the electronics industry due to its remarkably high electric conductivity, including the use in rechargeable batteries, microelectronic devices and sensors [116]. Electrochemical synthesis of polypyrrole can 
be achieved on copper electrodes, amongst others, where it grows into thin films acting as corrosion inhibitor $[117,118]$.

These examples illustrate that the interaction of pyrrole molecules with a metal surface is of great interest in many fields of science and technology. To understand the forces driving the formation of SAMs or the polymerisation process on a copper electrode, it is crucial to gain insight into the underlying mechanisms of diffusion of the molecules on the surface. Using helium-3 spin-echo (HeSE), the dynamics of this elementary building block, pyrrole, on $\mathrm{Cu}(111)$ can be studied to investigate inter-adsorbate interactions and to gain information about the adsorbate-substrate interactions in a sub-monolayer regime.

This chapter presents a combined HeSE and molecular dynamics study of pyrrole $/ \mathrm{Cu}(111)$ investigating the adsorbate dynamics at sub-monolayer coverages. First, a summary of the literature background about pyrrole adsorption on metal surfaces is given. Subsequently, experimental measurements of the dynamics of pyrrole $/ \mathrm{Cu}(111)$ are presented. Finally, detailed analysis is made using molecular dynamics simulations to reproduce the experimental data, supplemented by information from first-principles density functional theory calculations carried out by Sacchi and Jenkins from the Department of Chemistry at Cambridge.

\subsection{Literature background}

This section summarises the experimentally determined structure of a pyrrole molecule and looks into the literature on pyrrole adsorption on metal surfaces, in particular copper.

\subsubsection{The structure of pyrrole}

The geometric structure of pyrrole in the gas phase has been determined by Bak et al. using microwave spectroscopy [119]. The authors performed a study of pyrrole and five deuterated pyrroles. By comparing the transitions observed in the microwave spectra of the different species, and making an assumption about the $\mathrm{C}-\mathrm{H}$ bond lengths, they obtained a full description of the geometric structure of the molecule. A schematic picture of the molecule illustrating bond lengths and angles is shown in Figure 6.1. Due to the aromatic nature of the molecule, all atoms are in the same plane in its undistorted form. 
Compared to the geometry of $\mathrm{Cp}$ (cf. Figure 5.1) the N-C as well as $\mathrm{C}_{2}-\mathrm{C}_{3}$ and $\mathrm{C}_{4}-\mathrm{C}_{5}$ bonds are shortened while the $\mathrm{C}_{3}-\mathrm{C}_{4}$ bond is slightly elongated. The $\mathrm{C}-\mathrm{H}$ bonds are of similar length as the $\mathrm{Cp} \mathrm{C}-\mathrm{H}$ bonds while the $\mathrm{N}-\mathrm{H}$ bond is somewhat shorter. All angles within the fivefold ring are very similar to those of a regular pentagon, with the $\mathrm{C}-\mathrm{N}-\mathrm{C}$ angle increased, and the $\mathrm{C}-\mathrm{C}-\mathrm{C}$ angles reduced by $1^{\circ}$. Overall, the geometry of pyrrole does not deviate significantly from the fivefold symmetric structure of Cp. In addition, the masses of nitrogen and carbon atoms are similar, putting the centre of mass of pyrrole near the geometric centre of the 5-membered ring. Therefore, the small asymmetry is not expected to influence greatly the adsorption behaviour and diffusion of pyrrole.

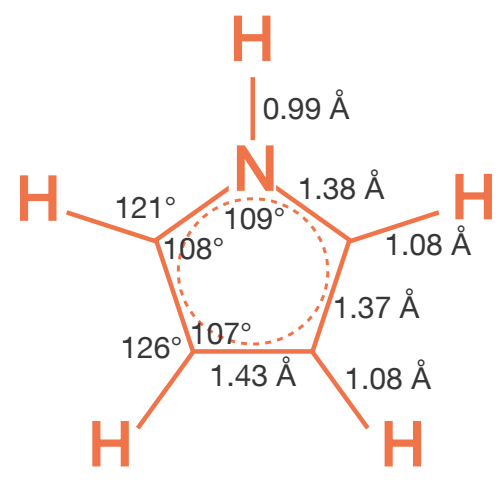

Figure 6.1: Schematic illustration of the structure of pyrrole (values for bond lengths and angles from [119]).

In another microwave spectroscopy study, the dipole moment of pyrrole has been determined as $\mu=1.74 \pm 0.02$ Debye or $0.36 \pm 0.01$ e $\AA$ [120]. This dipole is in the molecular plane of the molecule. Upon adsorption on a surface, the dipole typically creates an image dipole in the surface, giving a quadrupole with no net dipole moment, resulting in weak repulsive lateral interactions only.

\subsubsection{Pyrrole adsorption on copper surfaces}

Little is known in the literature about the adsorption of pyrrole on copper surfaces. A vibrational and temperature dependence study of pyrrole/ $\mathrm{Cu}(100)$ by Sexton [121] and a scanning tunnelling microscopy (STM) investigation of the same system by Gaudioso and Ho [122] are summarised below. 
Sexton conducted vibrational electron energy loss spectroscopy (EELS) and thermal desorption spectroscopy (TDS) for different exposures of pyrrole/ $\mathrm{Cu}(100)$ in a temperature range between $85 \mathrm{~K}$ and $300 \mathrm{~K}$ [121]. He found that pyrrole adsorbs molecularly and reversibly and shows three distinct peaks in TDS. Sexton identified the lowest temperature TDS peak as a multilayer state, while two further peaks of approximately equal integrated area, at $250 \mathrm{~K}$ and $200 \mathrm{~K}$, could be explained by a bi-layer formation of pyrrole molecules at lower coverages. The highest temperature peak at $250 \mathrm{~K}$ was attributed to a monolayer of flat-lying molecules. Assuming first order reaction kinetics, the author calculated the heat of adsorption for the monolayer as $64 \mathrm{~kJ} / \mathrm{mol}(0.66 \mathrm{eV})$, which is low compared to that of strongly chemisorbed molecules (typically $\geq 75 \mathrm{~kJ} / \mathrm{mol}(0.78 \mathrm{eV})$ ) and thus implies that bonding of pyrrole happens through weak $\pi$-bonding and van der Waals forces. The peak at $200 \mathrm{~K}$, which corresponds to a heat of adsorption of $51 \mathrm{~kJ} / \mathrm{mol}$ $(0.53 \mathrm{eV})$, was interpreted as a second layer of pyrrole molecules. In this state, the adsorbate system showed a more complicated vibrational spectrum than the monolayer state. From the attenuation of certain peaks in the EELS spectra of the monolayer, Sexton concluded an orientation with the molecular ring parallel to the surface in the monolayer, while the appearance of new peaks in the EELS spectra of the $200 \mathrm{~K}$ TDS state was interpreted as adsorption with the molecular plane tilted with respect to the surface in the second layer.

Gaudioso and Ho reported an STM study of pyrrole/Cu(100) at $9 \mathrm{~K}$ [122]. Comparing the apparent height of the molecules with those of other similar ring components on the same substrate, the authors suggested a flat-lying, $\pi$-bonded configuration for pyrrole, in agreement with Sexton's monolayer state [121]. In an inelastic electron tunnelling spectroscopy (IETS) study, no vibrational modes were observed for pyrrole.

\subsubsection{Pyrrole adsorption on other transition metal surfaces}

The adsorption behaviour of pyrrole has also been studied on various other transition metal surfaces, including $\mathrm{Ni}(100)$ [123], $\operatorname{Pd}(111)$ [124-126], Pt(111) [127], Rh(111) [128], and $\operatorname{Mo}(110)$ [129,130]. Most commonly, pyrrole is found to adsorb in a flat-lying geometry at low coverages, often moving to a tilted geometry at higher coverages - just as on $\mathrm{Cu}(100)$ [121] - or decomposing on the surface.

Schoofs and Benzinger studied the adsorption and decomposition of pyrrole/ $\mathrm{Ni}(100)$ using TDS, Auger electron spectroscopy (AES) and reflection absorption infrared spec- 
troscopy (RAIRS) [123]. In a similar way to its behaviour on $\mathrm{Cu}(100)$ [121], RAIRS data imply an adsorption geometry with the ring approximately parallel to the surface at $200 \mathrm{~K}$. The authors further conducted TDS experiments using two different heating rates, $8 \mathrm{~K} / \mathrm{s}$ and $45 \mathrm{~K} / \mathrm{s}$, and observed both molecular desorption and decomposition with a transition at room temperature. For both heating rates, a molecular desorption peak at $235 \mathrm{~K}$ was found. Above $300 \mathrm{~K}$, desorption of molecular hydrogen, $\mathrm{H}_{2}$, was observed using the lower heating rate, while desorption of $\mathrm{H}_{2}$ as well as hydrogen cyanide (HCN) and ammonia $\left(\mathrm{NH}_{3}\right)$ occured at $570 \mathrm{~K}$ for the higher heating rate. In both cases, AES showed carbon and nitrogen residue on the $\mathrm{Ni}(100)$ surface.

Pyrrole/Pd(111) was investigated using X-ray photoelectron spectroscopy (XPS), TDS and high resolution electron energy loss spectroscopy (HREELS) by Baddeley et al. [124]. In analogy to the observations for pyrrole on $\mathrm{Cu}(100)$ and $\mathrm{Ni}(100)$ [121,123], molecular adsorption of pyrrole was found on $\operatorname{Pd}(111)$, with HREEL spectra suggesting an adsorption geometry almost parallel to the surface for low coverages at $200 \mathrm{~K}$. With increasing coverage, however, pyrrole tilts away from the surface and a large fraction of molecules undergo $\mathrm{N}-\mathrm{H}$ bond cleavage. Close to the saturation coverage, HREELS experiments provide evidence for co-adsorption of strongly tilted molecular pyrrole $(\sim 13 \pm 7 \%)$ and pyrrolyl $\left(\mathrm{C}_{4} \mathrm{H}_{4} \mathrm{~N}\right)$ species $(\sim 87 \pm 7 \%)$. TDS experiments show partial molecular desorption of pyrrole from the monolayer in a single peak at $165 \mathrm{~K}$ while decomposition of the species remaining on the surface resulted in desorption of $\mathrm{HCN}$ above $500 \mathrm{~K}$ and $\mathrm{H}_{2}$ in two desorption peaks at $350 \mathrm{~K}$ and $475 \mathrm{~K}$.

Using laser-induced thermal desorption with Fourier transform mass spectrometry (LITD/FTMS) Caldwell and Land studied the reaction mechanism underlying the pyrrole decomposition on $\operatorname{Pd}(111)$ [126]. With a heating rate of $10^{10} \mathrm{~K} / \mathrm{s}$, LITD produces "snapshots" of the molecular composition at a specific coverage. The authors report that decomposition of pyrrole occurs at $\sim 230 \mathrm{~K}$ with $\mathrm{HCN}$ as the only reaction product detected in the mass spectrometer. They propose a reaction mechanism where molecular adsorption occurs at low temperatures, followed by $\mathrm{N}-\mathrm{H}$ bond cleavage as the temperature increases, and ring opening above $300 \mathrm{~K}$ to form $\mathrm{HCN}$ which remains intact up to $500 \mathrm{~K}$, in good agreement with the findings of Baddeley et al.

Using extended Hückel molecular orbital theory, Futaba and Chiang calculated predictions of STM images of pyrrole/Pd(111) [125]. Their calculations show bridge sites as the preferred adsorption sites. 
Tourillon et al. performed a near edge X-ray absorption fine structure (NEXAFS) study of pyrrole/Pt(111) to investigate the coverage-dependent adsorption geometry of pyrrole [127]. Contrary to the observations made for pyrrole adsorption on $\mathrm{Cu}(100)$, $\mathrm{Ni}(100)$, and $\operatorname{Pd}(111)[121,123,124]$, the authors report an ordered phase of pyrrole with the molecular plane oriented normal to the surface at $84 \mathrm{~K}$, bonding through the $\mathrm{N}$ atom. For coverages below that of the ordered layer and for multilayers they found disordered structures resulting in NEXAFs spectra independent of polarisation. No pyrrole decomposition on $\mathrm{Pt}(111)$ was observed.

Netzer et al. carried out experimental studies of pyrrole/Rh(111), combining angle resolved UV photoelectron spectroscopy (ARUPS), low energy electron diffraction (LEED), TDS and work function measurements [128]. The authors concluded that, at low temperatures, pyrrole bonds to the surface through $\pi$-bonding, resulting in a geometry parallel to the surface as reported on many other metal surfaces. In TDS measurements only a small fraction of molecules desorbs molecularly with a desorption peak at $360 \mathrm{~K}$, while the majority decompose. Between 150 and $300 \mathrm{~K}$, the authors reported $\mathrm{N}-\mathrm{H}$ bond cleavage leading to reaction limited desorption of molecular hydrogen and interaction of the free $\mathrm{N}$ lone pair electrons with the surface. The pyrrolyl species further decomposes into $\mathrm{HCN}$, which desorbs at $420 \mathrm{~K}$, and surface carbon.

In a more recent experimental and DFT study, Abdallah and Nelson investigated the pyrrole/Mo(110) system $[129,130]$. The authors suggest that pyrrole adsorbs flatlying through its $\pi$-orbitals at low coverage and coexisting flat-lying and slightly tilted molecules at higher exposures. TDS measurements show a multilayer desorption peak at $248 \mathrm{~K}$ and desorption of the monolayer at $345 \mathrm{~K}$ to $354 \mathrm{~K}$, corresponding to an activation energy of $90.2 \mathrm{~kJ} / \mathrm{mol}$ to $92.6 \mathrm{~kJ} / \mathrm{mol}(0.93 \mathrm{eV}$ to $0.96 \mathrm{eV})$. Auger analysis after desorption reveals residual carbon and nitrogen on the surface, in the ratio 4:1 at low exposures and 2:1 at high exposures. However, no decomposition products were detected in TDS, which implies that while the adsorbate does not fully desorb molecularly, $\mathrm{Mo}(110)$ does not catalyse a similar reaction to $\mathrm{HCN}$ and $\mathrm{H}_{2}$ as that on $\operatorname{Pd}(111), \operatorname{Rh}(111)$ and $\mathrm{Ni}(100)[123,124,128]$. Performing DFT calculations, Abdallah and Nelson found a flat-lying adsorption geometry with the pyrrole ring centred over a hollow site. Increasing the coverage results in co-adsorption of molecules with the ring parallel to the surface and slightly tilted ones due to lateral adsorbate interactions. Molecular pyrrole does not bond in a strongly tilted or perpendicular orientation but abstraction of a hydrogen atom makes these configurations more favourable. 


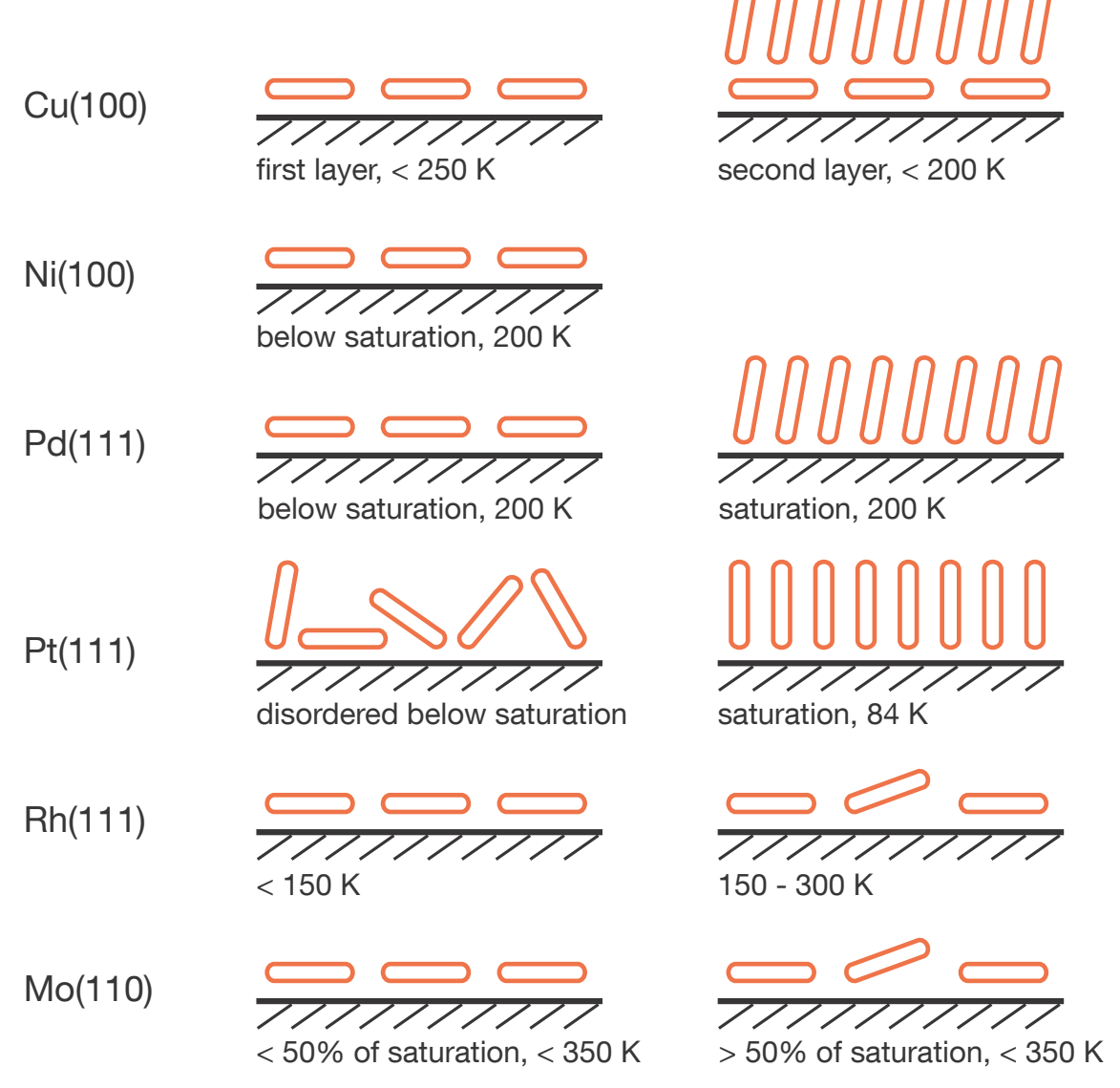

Figure 6.2: Summary of the pyrrole adsorption configurations reported in the literature for $\mathrm{Ni}(100)$ [123], $\operatorname{Pd}(111)$ [124-126], $\operatorname{Pt}(111)$ [127], $\operatorname{Rh}(111)$ [128], and $\operatorname{Mo}(110)[129,130]$.

To summarise, pyrrole adsorption has been studied on a range of transition metal surfaces. Figure 6.2 compares the adsorption geometry of pyrrole on different substrates. It is found that pyrrole commonly adopts a flat-lying geometry at low coverages, except on $\mathrm{Pt}(111)$ where a disordered sub-monolayer geometry was reported. It is therefore reasonable to assume that pyrrole adsorbs with its molecular ring parallel to the surface on $\mathrm{Cu}(111)$, as confirmed by DFT calculations (cf. Section 6.5). Upon increasing the temperature, the adsorbate desorbs molecularly from $\mathrm{Cu}(100)$ and $\mathrm{Pt}(111)$, while $\mathrm{Ni}(100)$, $\operatorname{Pd}(111)$ and $\mathrm{Rh}(111)$ are more reactive and catalyse decomposition resulting in desorption of $\mathrm{H}_{2}$ and $\mathrm{HCN}$, as well as residual surface carbon. $\mathrm{Mo}(110)$ also catalyses decomposition, however no desorption of decomposition products was detected and surface carbon and nitrogen was reported instead. 


\subsection{Experimental methods}

The dynamics and diffraction measurements presented in this chapter were performed in the HeSE spectrometer, described in Chapter 2, using a beam energy between $7.5 \mathrm{meV}$ and $8.5 \mathrm{meV}$. In addition, TDS experiments were carried out in MiniScat, using a Pfeiffer quadrupole mass spectrometer (cf. Chapter 3, "original setup"). The background pressure in the scattering chamber was $2 \cdot 10^{-11}$ mbar in the HeSE spectrometer (Leybold Ionivac ion gauge [48]) and $3 \cdot 10^{-10} \mathrm{mbar}$ in MiniScat (Edwards ion gauge [49]).

In both instruments, a single crystal $\mathrm{Cu}(111)$ sample (Surface Preparation Laboratory, Netherlands) was mounted inside the UHV chamber on a six-axis manipulator. The crystal was cleaned by $\mathrm{Ar}^{+}$sputtering $(800 \mathrm{eV}, 10 \mu \mathrm{A}, 20$ minutes at $300 \mathrm{~K})$ and annealing to $800 \mathrm{~K}$ (30 seconds) and aligned azimuthally using the known diffraction pattern of a CO overlayer [27]. Surface cleanliness was monitored using the specular reflectivity of the helium beam, which was $\geq 35 \%$.

At room temperature, pyrrole readily polymerises, as apparent through the change in colour from a clear liquid to a red-brown colour. Pyrrole (Sigma-Aldrich, reagent grade $98 \%$ ) was therefore kept in a fridge to avoid polymerisation and only small quantities were connected to the vacuum system and exchanged regularly. It was then purified by repeated freeze-thaw cycles in high vacuum and dosed by backfilling the UHV chamber through a leak valve. The purity of the dosed pyrrole was monitored using a quadrupole mass spectrometer.

The sample temperature during dosing was $160 \mathrm{~K}$, except for the experiments of the dynamics at $200 \mathrm{~K}$ when the sample was also prepared at $200 \mathrm{~K}$, and TDS experiments where dosing was performed at $100 \mathrm{~K}$. Once prepared, the adsorbate dynamics remained consistent for at least 24 hours in the temperature range between 100 and $180 \mathrm{~K}$, implying that the overlayer is stable and no significant contamination occurs. Since desorption begins just beyond $200 \mathrm{~K}$, the surface had to be prepared more frequently for measurements at this temperature. Pyrrole adsorbs reversibly, hence the crystal could be cleaned by flashing to $400 \mathrm{~K}$, recovering the reflectivity of the crystal to within $\geq 95 \%$. In addition, the crystal was cleaned by sputtering and annealing once every two to three days. 


\subsection{Adsorption behaviour}

This section looks at the adsorption behaviour of pyrrole/ $\mathrm{Cu}(111)$, studying uptake curves, diffraction patterns and thermal desorption spectra. The aim is to fully understand the nature of the adsorbate species on the surface before studying its motion.

Two typical uptake curves for pyrrole/Cu(111), measured on the HeSE apparatus, are shown in Figure 6.3, where the blue line represents an uptake curve at $160 \mathrm{~K}$ and the red line one at $200 \mathrm{~K}$. At the lower temperature, the curve first falls below a straight line for exposures up to about $2 \cdot 10^{-5}$ mbar s, then continues in an approximately linear fashion before curving down rapidly at an exposure of $3.7 \cdot 10^{-5}$ mbar s. The non-linear behaviour at low exposures indicates repulsive interactions between pyrrole adsorbates [15]. The deviation from a straight line is much more pronounced in the case of pyrrole/ $\mathrm{Cu}(111)$ than that observed for $\mathrm{Cp} / \mathrm{Cu}(111$ ) (cf. Section 5.3), implying that pyrrole adsorbates behave in a more strongly repulsive manner than $\mathrm{Cp}$, as will be confirmed in the dynamics measurements. The change in uptake behaviour at $2 \cdot 10^{-5}$ mbar s implies a phase transition, and could indicate a change in adsorption geometry, such as for example moving from first layer flat-lying molecules to the second layer of tilted molecules observed on $\mathrm{Cu}(100)$ [121]. The final curve towards vertical, at an exposure of $3.7 \cdot 10^{-5} \mathrm{mbar} \mathrm{s}$, is indicative of 3D island growth on the surface, resulting in disorder and thus attenuation of the specular helium reflectivity.

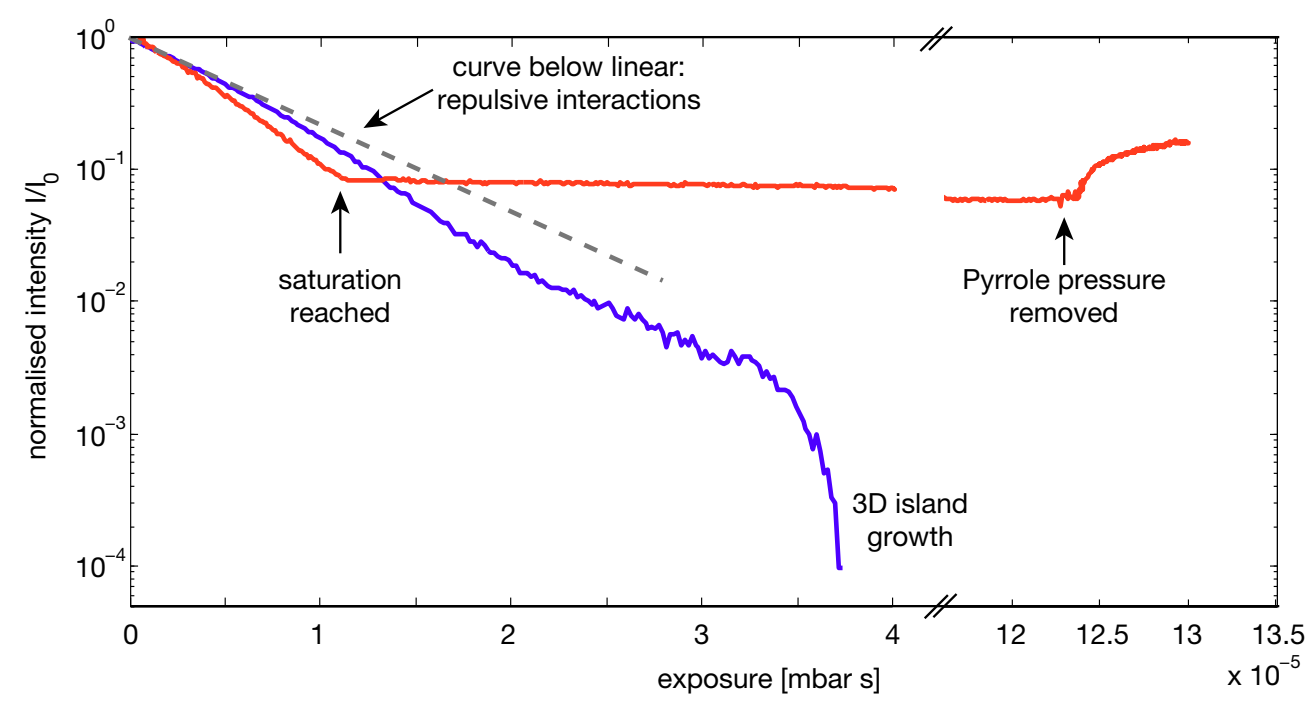

Figure 6.3: Uptake curves for pyrrole on $\mathrm{Cu}(111)$ at $160 \mathrm{~K}$ (blue) and $200 \mathrm{~K}$ (red), measured on the HeSE spectrometer. A grey dashed line is added to guide the eye and demonstrate the deviation from linear behaviour. 
Slightly different behaviour is observed when the uptake experiment is repeated at $200 \mathrm{~K}$, shown in red. Up to $\sim 1 \cdot 10^{-5}$ mbar s exposure, the uptake curve at $200 \mathrm{~K}$ also falls below a straight line, curving down even more quickly than that at $160 \mathrm{~K}$. This apparent strengthened repulsive behaviour at $200 \mathrm{~K}$ could be interpreted as an increased scattering cross section observed by the helium beam at the higher temperature due to the increase in inelastic scattering from the pyrrole as the amplitude of its thermally induced vibrations increases. At $\sim 1 \cdot 10^{-5}$ mbar s the $200 \mathrm{~K}$ curve reaches a constant level. When the pyrrole overpressure is removed (right-hand side of the Figure), some of the adsorbed pyrrole desorbs from the surface and a new equilibrium coverage is obtained at an intensity of approximately $\mathrm{I}_{0} / 5$. The change in intensity after removing the pressure implies that an equilibrium between adsorption and desorption was reached at $\sim 1 \cdot 10^{-5}$ mbar s, resulting in nearly constant helium intensity, and the equilibrium shifts when the valve is closed, causing partial desorption. The small reduction in intensity between $\sim 1 \cdot 10^{-5}$ mbar s and the point where the valve was closed is due to slight instabilities in the pyrrole overpressure in the UHV chamber, shifting the adsorption-desorption equilibrium to a higher coverage. A lower saturation coverage at $200 \mathrm{~K}$ than at $160 \mathrm{~K}$ is in agreement with the TDS measurements presented below.

The HeSE experiments presented in Section 6.4 have been performed at two different sample temperatures, $200 \mathrm{~K}$ and $160 \mathrm{~K}$. The $160 \mathrm{~K}$ measurements were recorded for three different coverages, corresponding to a specular attentuation of $\mathrm{I}_{0} / 3.4, \mathrm{I}_{0} / 9.0$, and $\mathrm{I}_{0} / 20$. Looking at the uptake curve shown in blue in Figure 6.3, these coverages are all in the regime where strong repulsive interactions are present, implying that the molecules do not form islands or multilayers on the surface. At $200 \mathrm{~K}$, only the lowest coverage was studied ${ }^{1}$.

Diffraction scans performed at $160 \mathrm{~K}$ for pyrrole overlayers of the three coverages described above are shown in Figure 6.4 (A). The lowest coverage scan is plotted in blue, the medium coverage scan in red and the highest coverage one in green. The diffraction pattern for the medium coverage has been studied along two azimuthal directions, $<1 \overline{1} 0>$ and $<11 \overline{2}>$. As is apparent from the two scans presented in the Figure, as well as several intermediate angles $\left(2^{\circ}, 5^{\circ}\right.$, and $7^{\circ}$ off the $<11 \overline{2}>$ azimuth; not shown $)$ the diffraction scans do not show azimuthal dependence. This implies that all observed features are diffraction rings rather than diffraction peaks and thus no regular overlayer structure is formed.

\footnotetext{
${ }^{1}$ The medium and high coverages can only be obtained at $200 \mathrm{~K}$ with a constant pyrrole overpressure
} in the UHV chamber, apparent by the signal rise on the right-hand side in Figure 6.3). 

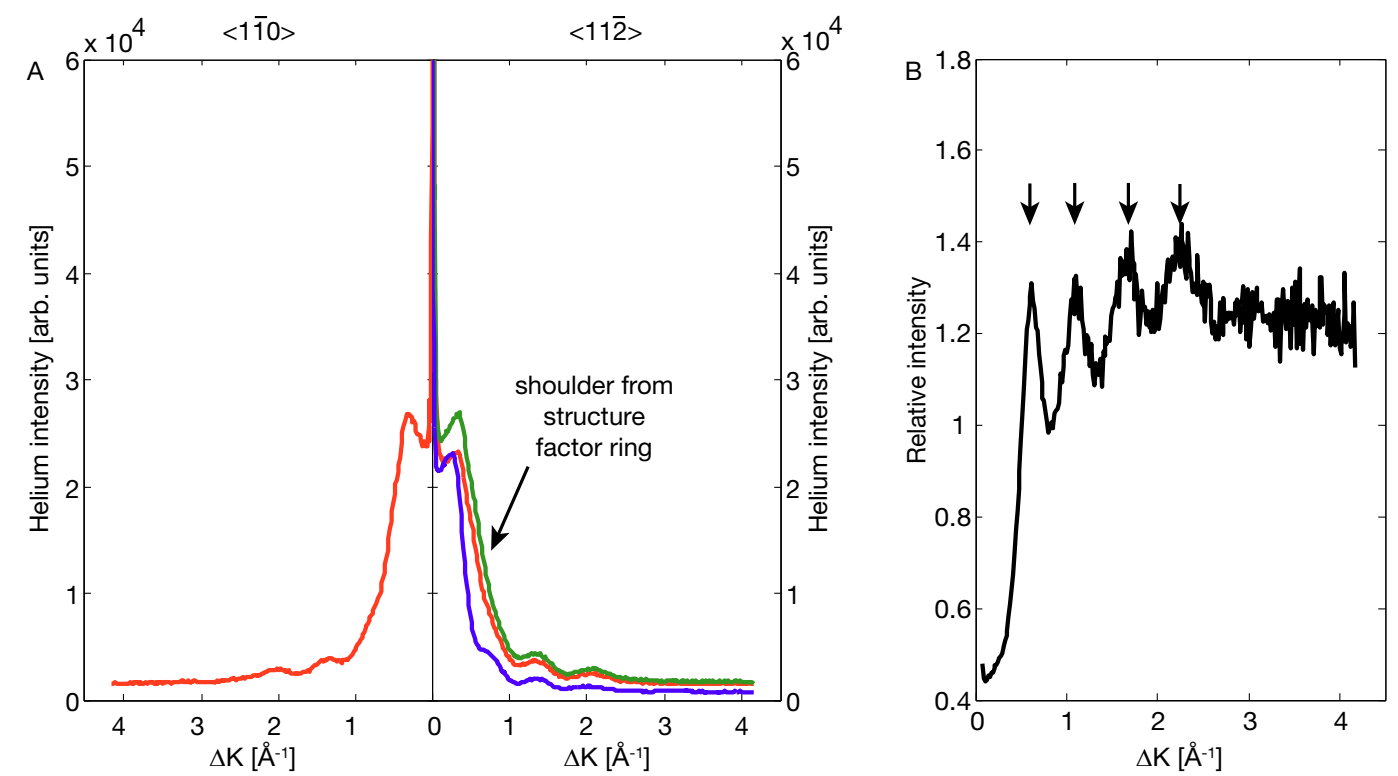

Figure 6.4: (A) Diffraction scans of pyrrole/Cu(111) at $160 \mathrm{~K}$ showing the three coverages used in HeSE measurements, $\mathrm{I}_{0} / 3.4$ (blue), $\mathrm{I}_{0} / 9.0$ (red), and $\mathrm{I}_{0} / 20$ (green). Dividing the high coverage diffraction scan (green) by the low coverage scan (blue) results in the black curve shown in (B), revealing additional, coverage dependent, features.

As shown in Equation 2.2, the total scattering amplitude is the product of a form factor, $F$, which is the property of the molecule and thus independent of coverage, and a structure factor, $S_{a}$, which contains information about the distribution of the molecules on the surface and therefore varies with coverage. In Figure $6.4(\mathrm{~A})$, diffraction rings can be identified at $0.3 \AA^{-1}, 0.7 \AA^{-1}, 1.3 \AA^{-1}$ and $2.0 \AA^{-1}$, all of approximately constant momentum transfer positions with varying coverage. These coverage-independent rings are thus form factor rings, i.e. they are due to the structure within the pyrrole molecules [9]. Although the peak positions do not change with coverage, a change in the overall shape of the diffraction scans can be observed (e.g. in the shoulder marked by an arrow). To extract the coverage dependent information from the data, the highest coverage diffraction scan (green) has been divided by the scan for the lowest coverage (blue), which results in the curve shown in Figure 6.4 (B). A series of peaks is apparent (arrows) which occur at multiples of $0.6 \AA^{-1}$. Since these features appear when dividing high coverage data by low coverage data, they are clearly coverage dependent and can be assigned as structure factor rings.

In order to further characterise the adsorption behaviour of pyrrole/ $\mathrm{Cu}(111)$, uptake, diffraction and TDS experiments were performed on MiniScat. Diffraction measurements 
in the same temperature range as the ones presented above show good agreement between the two instruments. After cooling the sample to $50 \mathrm{~K}$ using liquid helium the diffraction pattern does not change significantly, and most importantly no discreet diffraction peaks are observed, implying that no periodic overlayer structures are formed at temperatures $\geq 50 \mathrm{~K}$. The results of the TDS study are shown in Figure 6.5, together with a MiniScat uptake curve indicating the exposure at which the different desorption measurements were started. Note that all uptake curves are presented using the pressure as read on the respective gauges, causing inaccuracies when comparing HeSE spectrometer and MiniScat data, i.e. Figures 6.3 and 6.5 should not be compared quantitatively but only in the shape of the curve. All TDS experiments are performed by heating the sample from $100 \mathrm{~K}$, where pyrrole was adsorbed, to approximately $500 \mathrm{~K}$ at a heating rate of $3 \mathrm{~K} / \mathrm{s}$, monitoring the signal of mass $67 \mathrm{amu}$ in a quadrupole mass spectrometer.

The bottom left panel in Figure 6.5 shows a typical plot of the specular helium intensity during desorption. The reflectivity of the sample increases as pyrrole desorbs, reversing the effect observed during uptake. Comparing the helium signal before dosing and after desorption, it can be confirmed that the process is fully reversible and the helium signal recovers to within $95 \%$ of its original value on the clean substrate surface. At temperatures above $290 \mathrm{~K}$ the signal decreases, which can be due to Debye Waller attenuation or a small misalignment of the sample during heating. The peak at $290 \mathrm{~K}$ implies that pyrrole desorption from the sample is complete at that temperature, suggesting that the contribution to the TDS spectra (panels top and centre left) above $290 \mathrm{~K}$ is due to desorption from the manipulator and sample mount and should thus be ignored (cf. grey dashed line).

Below $290 \mathrm{~K}$, four peaks can be identified at $165 \mathrm{~K}, 190 \mathrm{~K}, 220 \mathrm{~K}$ and a broad peak around $260 \mathrm{~K}$. The two lowest coverage curves (orange and red) only exhibit one broad peak at $260 \mathrm{~K}$, with desorption starting at $200 \mathrm{~K}$. At a specular attenuation between $\mathrm{I}_{0} / 41$ and $\mathrm{I}_{0} / 500$ (purple, blue, cyan and green curves) a growing second peak at $220 \mathrm{~K}$ is observed. Comparing the results to the TDS experiments of pyrrole/ $\mathrm{Cu}(100)$ reported by Sexton [121], it is reasonable to assume that the $260 \mathrm{~K}$ peak is due to desorption from a monolayer, while the $220 \mathrm{~K}$ peak arises from a second layer with different adsorption geometry, confirming the interpretation of the changing shape in the uptake curve at $160 \mathrm{~K}$ (cf. Figure 6.3). At higher exposures (black curve in top left panel), two additional desorption peaks appear in the thermal desorption spectrum, one at $190 \mathrm{~K}$ and one at $165 \mathrm{~K}$. These peaks can be assigned to multilayer desorption. A structural change seems to occur in the multilayer at temperatures between 190 and $165 \mathrm{~K}$, creating two peaks 

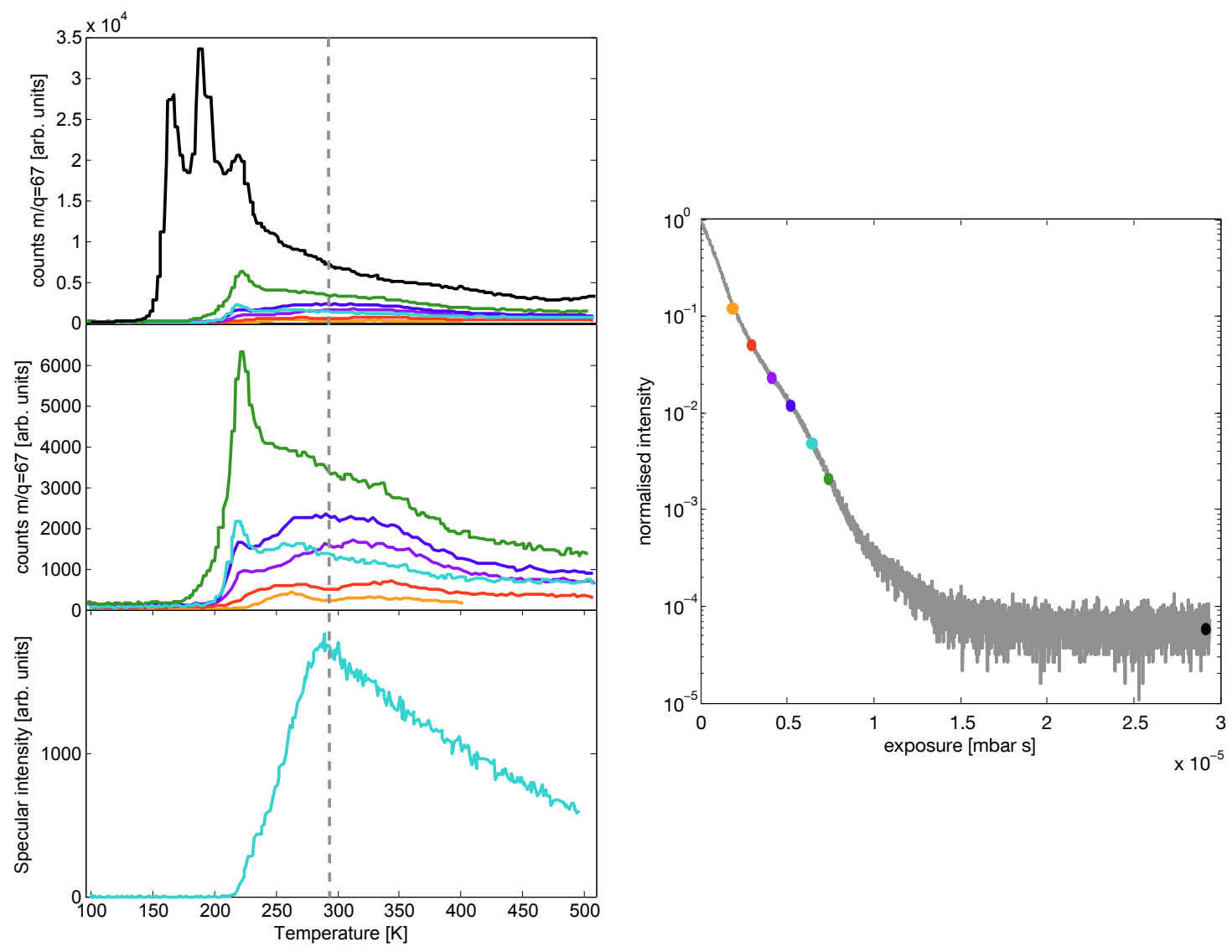

Figure 6.5: Thermal desorption spectra for pyrrole/Cu(111) for a range of coverages. The black line in the top left panel shows desorption from a multilayer, the second panel represents a magnified view of desorption after smaller doses. The corresponding specular attenuation values are $\mathrm{I}_{0} / 8$ (orange), $\mathrm{I}_{0} / 20$ (red), $\mathrm{I}_{0} / 41$ (purple), $\mathrm{I}_{0} / 80$ (blue), $\mathrm{I}_{0} / 200$ (cyan), $\mathrm{I}_{0} / 500$ (green), as marked on the MiniScat uptake curve at $100 \mathrm{~K}$ shown in the right panel. In the bottom left panel the specular helium intensity is plotted as a function of temperature, showing increasing sample reflectivity as desorption progresses, peaking at $290 \mathrm{~K}$ (dashed line).

rather than a single multilayer peak. Further investigation would be needed, however, to determine the different adsorption geometries and bonding configurations corresponding to the two multilayer peaks.

All HeSE experiments were performed at specular attenuation levels that fall in the sub-monolayer regime $\left(\mathrm{I}_{0} / 3.4, \mathrm{I}_{0} / 9.0\right.$ and $\left.\mathrm{I}_{0} / 20\right)$. The position of the monolayer peak maximum is approximately constant with coverage, indicative of first order desorption kinetics. The Redhead equation [131] can be used to calculate the adsorption energy, E, 
via

$$
E=R T_{m}\left[\ln \left(\frac{\nu T_{m}}{\beta}\right)-3.64\right]
$$

where $R$ is the ideal gas constant, $T_{m}$ the temperature of the peak maximum in the desorption spectrum, $\beta=d T / d t$ the heating rate, and $\nu$ the pre-exponential factor for desorption (typically estimated as $10^{13} \mathrm{~s}^{-1}$ ). An adsorption energy of $66 \mathrm{~kJ} / \mathrm{mol}$ or $0.68 \mathrm{eV}$ is obtained for the monolayer peak.

To summarise, the coverages used in HeSE experiments are in the sub-monolayer regime. Pyrrole does not form regular overlayer structures on $\mathrm{Cu}(111)$. TDS experiments show a fully reversible adsorption process, with monolayer desorption starting above $200 \mathrm{~K}$.

\subsection{Measuring the surface dynamics}

This section describes HeSE experiments for pyrrole/ $\mathrm{Cu}(111)$, investigating the change of the lineshapes in the intermediate scattering function (ISF) with momentum transfer parallel to the surface, coverage and temperature. In addition, measurements were performed along two principal high symmetry directions, $<1 \overline{1} 0>$ and $<11 \overline{2}>$, to map out the two-dimensional motion of pyrrole. Note that all measurements presented in this chapter are for positive momentum transfers. Corresponding measurements at negative momentum transfers have demonstrated identical results either side of the specular peak.

\subsubsection{Experimental lineshapes}

HeSE measurements of pyrrole/Cu(111) were performed at three different coverages, corresponding to a specular attenuation level of $\mathrm{I}_{0} / 3.4, \mathrm{I}_{0} / 9.0$, and $\mathrm{I}_{0} / 20$. Figures 6.6 and 6.7 show a series of typical ISFs at the low and medium coverages, respectively, with increasing momentum transfers from the top left to the bottom right panels. There are several features to be noted at various timescales.

At $\Delta \boldsymbol{K}<1.2 \AA^{-1}$ in the lowest coverage data, oscillatory features similar to those observed for $\mathrm{Cp} / \mathrm{Cu}(111$ ) (cf. Section 5.4.1) can be identified, marked with a label A in Figure 6.6. The intensity of these oscillations is much reduced at the medium coverage, as 
can be seen when comparing spectra at the same $\Delta \boldsymbol{K}$ position (e.g. centre left panel in Figure 6.6 and top right panel in Figure 6.7). Such a decrease in intensity with coverage suggests that the features are predominantly due to $\mathrm{Cu}(111)$ substrate phonons, as in the case of $\mathrm{Cp} / \mathrm{Cu}(111)$.

In addition to the oscillations, a fast decay can be observed at small $\Delta \boldsymbol{K}$, evident by a change in the shape of the ISF at approximately $0.5 \mathrm{ps}$ (label B). As seen for $\mathrm{Cp} / \mathrm{Cu}(111)$ (cf. Section 5.9), this fast decay is typically due to intracell diffusion which is related to the friction experienced by the adsorbate [98] and can be ignored in the analysis of the inter-cell adsorbate diffusion. Section 6.6 demonstrates that a cut-off point of 0.5 ps is in good agreement with the friction value from molecular dynamics simulations.

The behaviour at long times is due to inter-cell diffusion of pyrrole $/ \mathrm{Cu}(111)$ that occurs on longer timescales than intracell diffusion and is analysed by ignoring the oscillations and the fast decay. The shape of the ISF is dependent on the type of motion. Continuous random motion and jump diffusion exhibit exponentially decaying lineshapes, while ballistic motion shows an ISF of Gaussian form (cf. Section 2.4). The ISFs in the pyrrole $/ \mathrm{Cu}(111)$ data show a clear exponential decay, indicating that pyrrole moves either in continuous or hopping motion. The black lines in Figures 6.6 and 6.7 show single exponentials that describe the data from $0.5 \mathrm{ps}$, of the functional form $f(t)=a \cdot \exp (-\alpha t)+c$, where $a$ is the preexponential factor, $c$ the static component, and the decay constant $\alpha$ is the dephasing rate. The constant level, typically caused by static defects, is a diffraction pattern. Therefore, the ISF at $2.84 \AA^{-1}$ along $<11 \overline{2}>$ shows a significantly increased static level (labelled D in Figure 6.7) compared to the lineshapes at other momentum transfer values, which is due to this position coinciding with the diffraction condition for $\mathrm{Cu}(111)$ on $<11 \overline{2}>[40]$.

In order to gain insight into how well a single exponential function represents the data, the residual after subtracting the exponential from the experimental curve is investigated (cf. Figures 6.6 and 6.7). If the experimental lineshape follows a single exponential function, the residual should show random scattering around zero. Were the lineshapes a sum of several exponential functions [38], on the other hand, the residual after subtracting a single exponential would be expected to show deviations, with points systematically above and below zero. Overall, a single exponential represents the measured data well, although small deviations can be observed at momentum transfers between $0.2 \AA^{-1}$ and $0.6 \AA^{-1}$, marked with a label C. The origin of these small deviations will be discussed in Section 6.6. Further measurements at the high coverage as well as along the $<1 \overline{1} 0>$ 

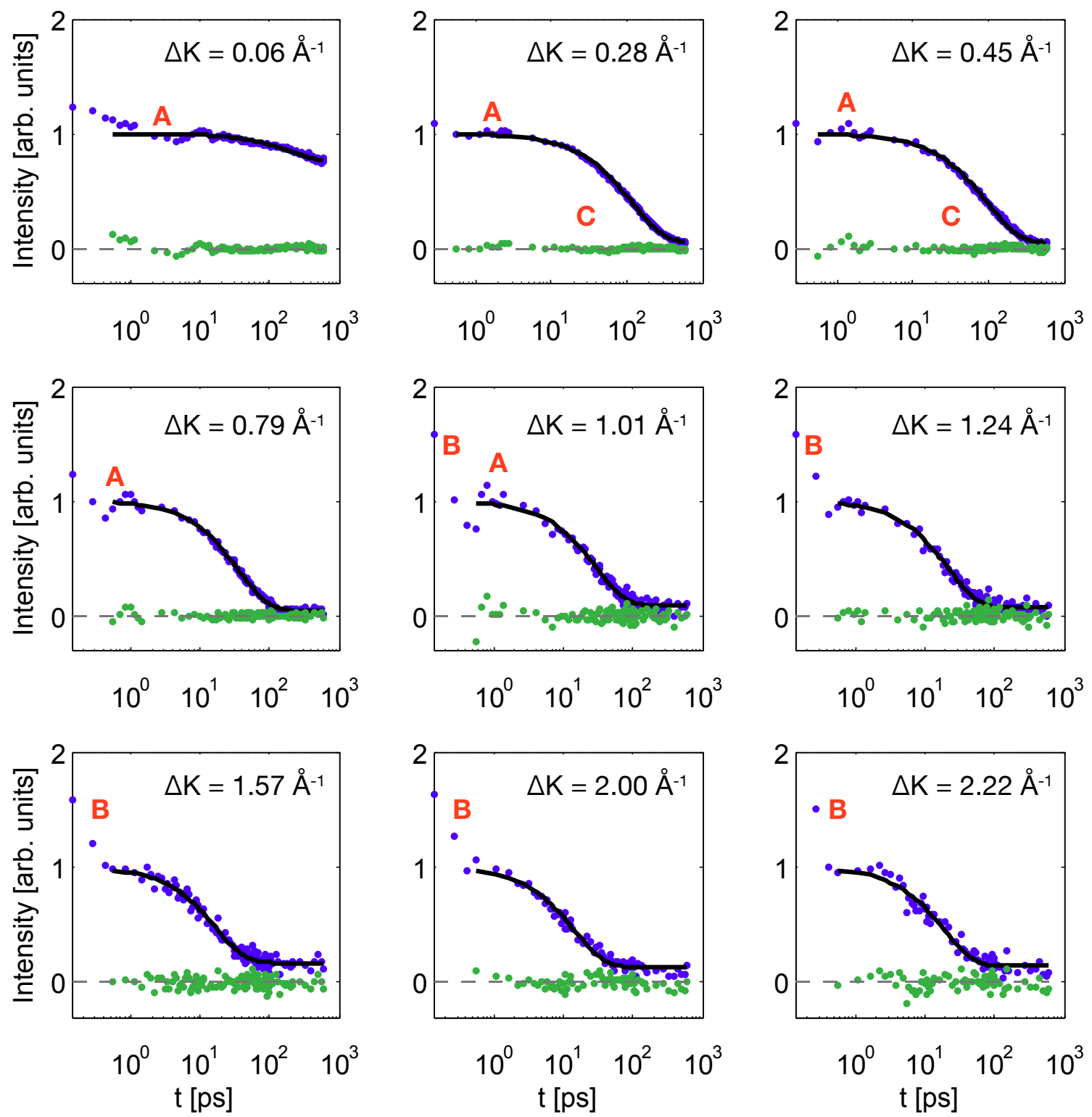

Figure 6.6: Polarisation measurements depicting the helium intensity in the detector as a function of spin-echo time, $t$, for the low coverage experiments of pyrrole $/ \mathrm{Cu}(111)$ along $<11 \overline{2}>$. The different panels show measurements at increasing momentum transfers. Experimental data are plotted as blue points, a single exponential of the form $f(t)=a \cdot \exp (-\alpha t)+c$ from $0.5 \mathrm{ps}$ is shown as a black line and the residual after subtracting the exponential from the data as green points. Red labels identify features mentioned in the text: oscillations (A), a fast decay (B) and deviations from zero residual $(\mathrm{C})$.

azimuth (not shown) confirm that a single exponential is a reasonable description of the experimental data. 

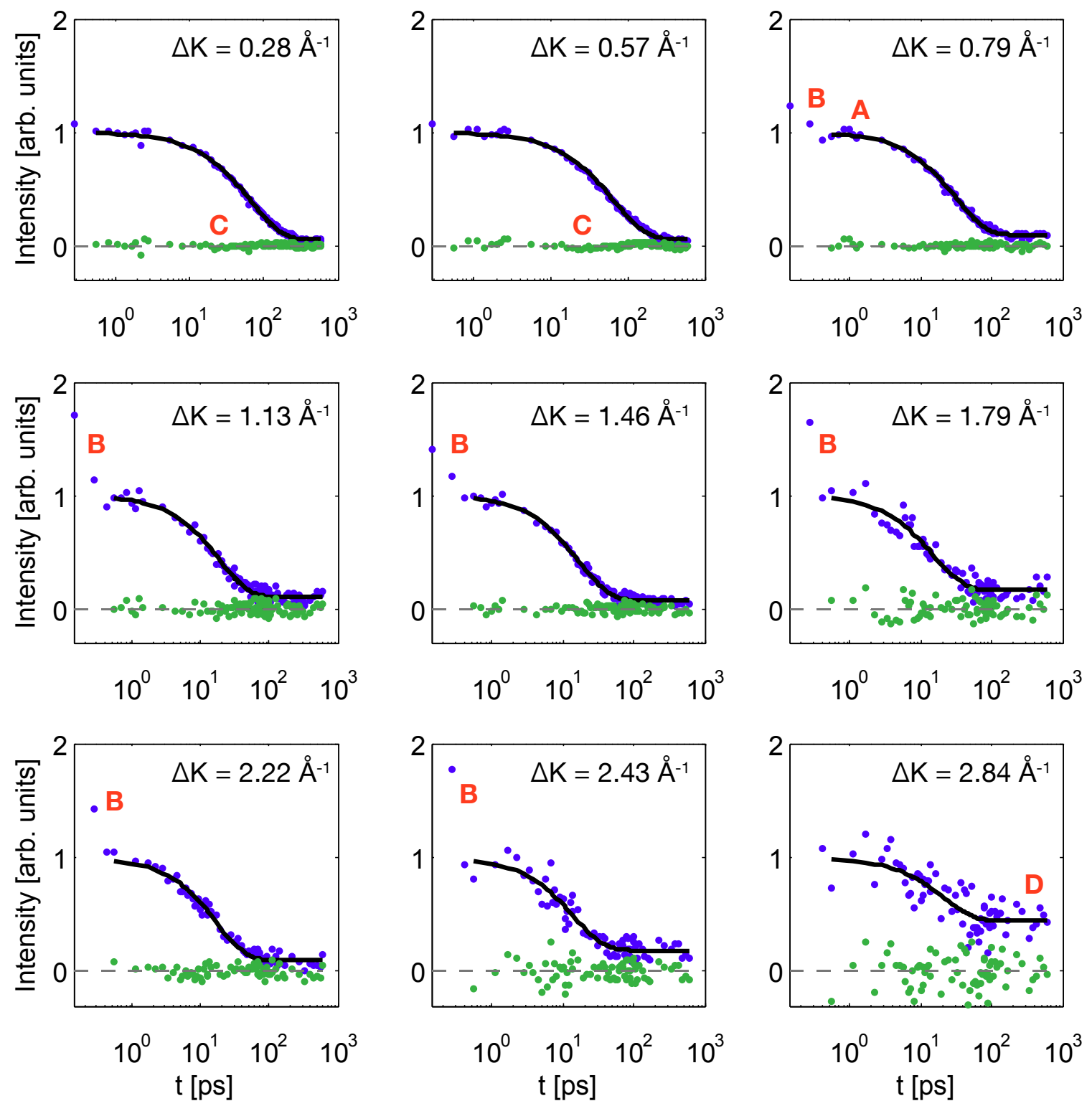

Figure 6.7: Polarisation measurements depicting the helium intensity in the detector as a function of spin-echo time, $t$, for the medium coverage experiments of pyrrole $/ \mathrm{Cu}(111)$ along $<11 \overline{2}>$. The different panels show measurements at increasing momentum transfers. Experimental data is plotted as blue points, a single exponential of the form $f(t)=a \cdot \exp (-\alpha t)+c$ from 0.5 ps is shown as a black line and the residual after subtracting the exponential from the data as green points. Red labels identify features mentioned in the text: oscillations (A), a fast decay (B), deviations from zero residual $(\mathrm{C})$ and a static level (D).

\subsubsection{Hopping over an energy barrier}

The experimental ISFs show exponentially decaying lineshapes, implying either continuous random motion or jump diffusion. Continuous motion would be over a flat potential energy 
landscape, while jump diffusion occurs on a corrugated potential energy surface (PES). These two cases can thus be distinguished by determining whether pyrrole experiences an activation barrier to diffusion.

As detailed in Chapter 5, Arrhenius' law predicts the temperature dependence of the dephasing rate $\alpha$ for activated processes as

$$
\alpha=\alpha_{0} \cdot \exp \left(-\frac{E_{a}}{k_{B} T}\right)
$$

where $E_{a}$ is the activation energy for diffusion, $T$ the sample temperature, $k_{B}$ the Boltzmann constant and $\alpha_{0}$ is the pre-exponential factor describing the jump attempt frequency. Plotting $1 / T$ versus $\ln \alpha$ gives a straight line with gradient $-E_{a} / k_{B}$ and intercept $\ln \alpha_{0}$. To determine the apparent activation barrier for pyrrole/ $\mathrm{Cu}(111)$, temperature dependent measurements at fixed momentum transfer were performed.

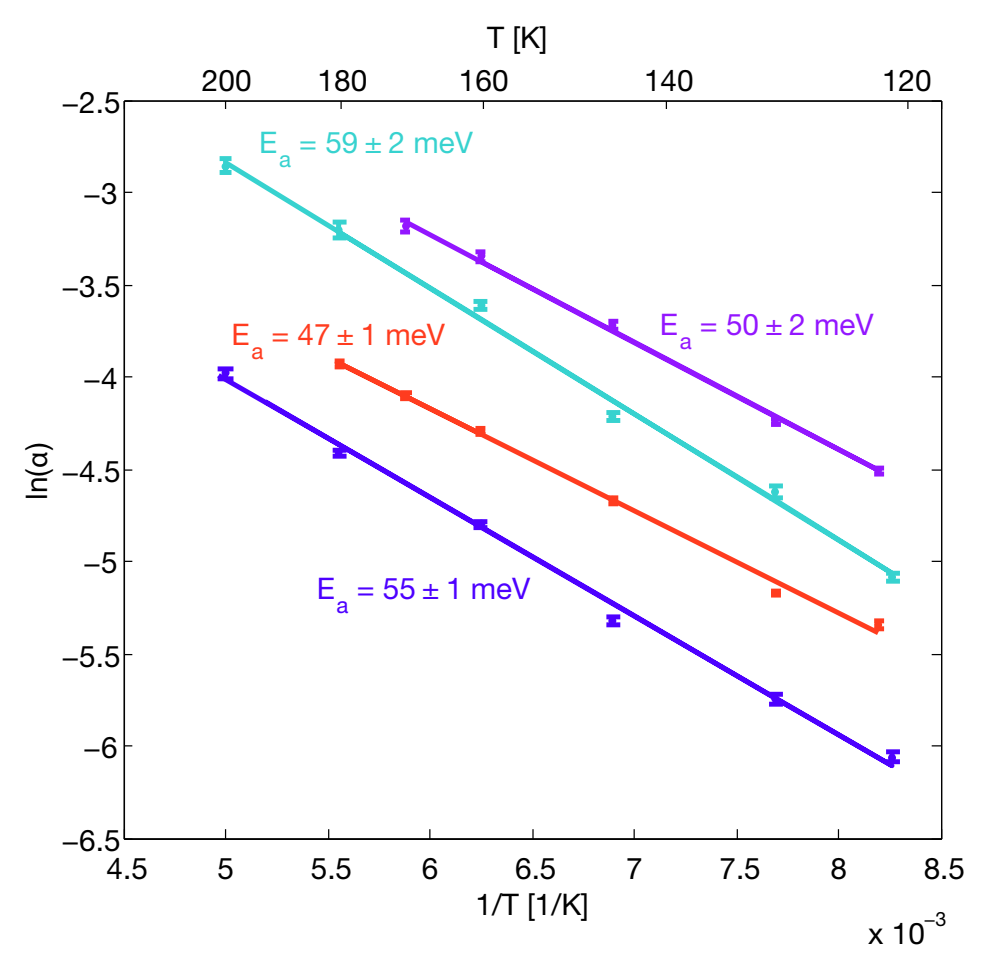

Figure 6.8: Arrhenius plots for pyrrole/Cu(111) show the apparent activation energy at different coverages and momentum transfer values, recorded at temperatures between $121 \mathrm{~K}$ and $200 \mathrm{~K}$. Low coverage data at $0.4 \AA^{-1}$ and $0.9 \AA^{-1}$ is shown in blue and cyan, respectively, medium coverage at $0.4 \AA^{-1}$ and $0.9 \AA^{-1}$ in red and purple. 
Figure 6.8 shows Arrhenius plots for two positions in $\Delta \boldsymbol{K}$ space at two different coverages. All four curves show a strong temperature dependence, indicating that the diffusion process is activated. It can therefore be concluded that pyrrole moves in jump diffusion across the $\mathrm{Cu}(111)$ surface.

The apparent Arrhenius activation energies at the low and medium coverage for $0.4 \AA^{-1}$ and $0.9 \AA^{-1}$ are summarised in Table 6.1. Overall, $E_{a}$ decreases at higher coverage and increases with $\Delta \boldsymbol{K}$, which is typically a symptom of strong repulsive interactions between the adsorbates. Combining the measurements, an effective activation energy of $53 \pm 4 \mathrm{meV}$ for pyrrole/ $\mathrm{Cu}(111)$ is obtained which is of the same order of magnitude as that for $\mathrm{Cp}$ on the same substrate (cf. Section 5.4.3), if somewhat larger.

\begin{tabular}{ccc}
\hline $\boldsymbol{\Delta} \boldsymbol{K}\left[\AA^{-\mathbf{1}}\right]$ & $\boldsymbol{E}_{\boldsymbol{a}}\left(\mathrm{I}_{\mathbf{0}} / \mathbf{3 . 4}\right)[\mathrm{meV}]$ & $\boldsymbol{E}_{\boldsymbol{a}}\left(\mathrm{I}_{\mathbf{0}} / \mathbf{9 . 0}\right)[\mathrm{meV}]$ \\
\hline 0.4 & $55 \pm 1$ & $47 \pm 1$ \\
0.9 & $59 \pm 2$ & $50 \pm 2$ \\
\hline
\end{tabular}

Table 6.1: Arrhenius activation energies for pyrrole $/ \mathrm{Cu}(111)$ at two different coverages, corresponding to a specular attenuation level of $\mathrm{I}_{0} / 3.4$ and $\mathrm{I}_{0} / 9.0$, and two positions in momentum transfer.

\subsubsection{Momentum transfer and coverage dependence of the dynamics}

Having determined that pyrrole diffusion is an activated process, the momentum transfer dependence of the dephasing rate $\alpha$ allows further investigation of the hopping motion.

Figure 6.9 shows the $\alpha(\Delta \boldsymbol{K})$ curves at $160 \mathrm{~K}$ for three different coverages along $<11 \overline{2}>$ (right panel), corresponding to specular attenuation levels of $\mathrm{I}_{0} / 3.4$ (blue), $\mathrm{I}_{0} / 9.0$ (red) and $\mathrm{I}_{0} / 20$ (green), and the medium coverage along $<1 \overline{1} 0>$ (left panel). Unlike the sinusoidal variation of the dephasing rate with momentum transfer predicted by simple jump diffusion models [35,38], the behaviour observed in the curves in Figure 6.9 is complex.

At low $\Delta \boldsymbol{K}$, the dip near $0.5 \AA^{-1}$ can be identified as de Gennes narrowing, described in Section 2.4.2, which together with a peak feature at around $0.3 \AA^{-1}$ is evidence for repulsive inter-adsorbate interactions. With increasing coverage and thus decreasing average adsorbate-adsorbate distance, the height of the peak becomes larger and the 


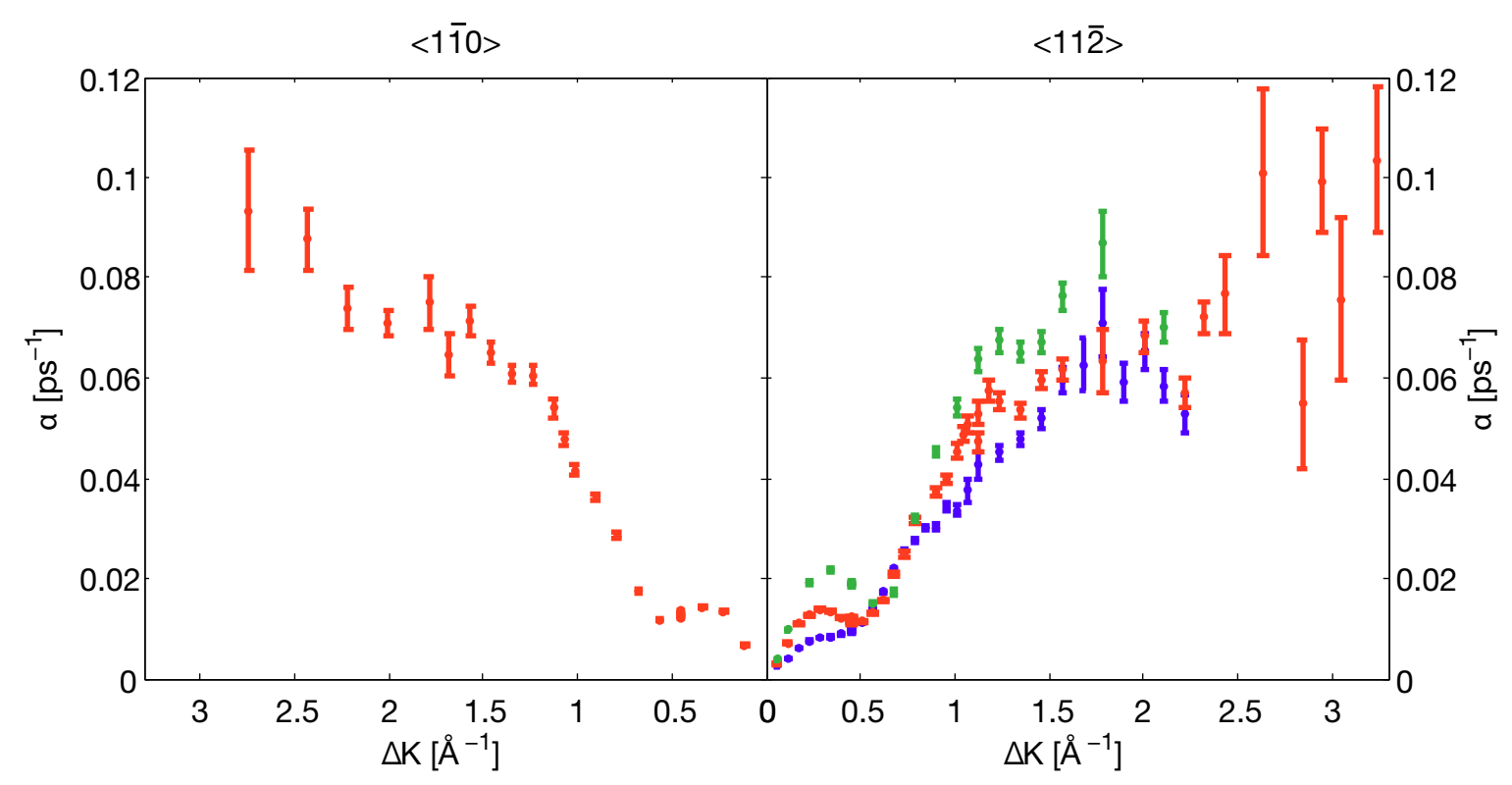

Figure 6.9: $\alpha(\Delta \boldsymbol{K})$ curves at $160 \mathrm{~K}$ are shown along the two azimuths $<1 \overline{1} 0>$ (left) and $<11 \overline{2}>$ (right). Blue dots represent measurements at $0.020 \mathrm{ML}$ coverage, red dots at $0.033 \mathrm{ML}$ and green dots at $0.052 \mathrm{ML}$.

position of the dip moves towards higher momentum transfers. The coverage for each curve can be determined from the momentum transfer position of the dip using Equation 2.24. Resulting coverages are summarised in Table 6.2 as fractions of a monolayer (ML), where $1 \mathrm{ML}$ is defined as one adsorbate molecule per substrate atom. Note that saturation coverages for molecular adsorbates are typically much smaller than $1 \mathrm{ML}$ due to the extended nature of the adsorbate species. Using the method described in Section 2.1 the coverage values are used to estimate the scattering cross section as $350 \AA^{2}$ which is larger than that determined for $\mathrm{Cp}\left(200 \AA^{2}\right)$ determined in Chapter 5. Comparing the $\alpha(\Delta \boldsymbol{K})$ curve for $0.033 \mathrm{ML}$ pyrrole with that for approximately 0.03 ML Cp on the same substrate (cf. Figure 5.14), the de Gennes feature is more pronounced in the case of pyrrole, indicating stronger repulsive interactions. This observation confirms the interpretation of the downward curvature observed during uptake of pyrrole, shown in Figure 6.3.

At high $\Delta \boldsymbol{K}$, an increase of the magnitude of the $\alpha(\Delta \boldsymbol{K})$ curve with coverage can be observed in the data along $<11 \overline{2}>$ (cf. Figure 6.9). The jump rate of pyrrole thus increases with coverage. In the Arrhenius measurements presented in Section 6.4.2, it is apparent that the jump rate also changes with temperature. Figure 6.10 compares an 


\begin{tabular}{ccc}
\hline specular attenuation & $\boldsymbol{\Delta} \boldsymbol{K}_{\boldsymbol{d i p}}\left[\AA^{-\mathbf{1}}\right]$ & coverage $\boldsymbol{\Theta}[\mathrm{ML}]$ \\
\hline $\mathrm{I}_{0} / 3.4$ & 0.40 & 0.020 \\
$\mathrm{I}_{0} / 9.0$ & 0.52 & 0.033 \\
$\mathrm{I}_{0} / 20$ & 0.65 & 0.052 \\
\hline
\end{tabular}

Table 6.2: Pyrrole coverages estimated from the position of the de Gennes narrowing dip.
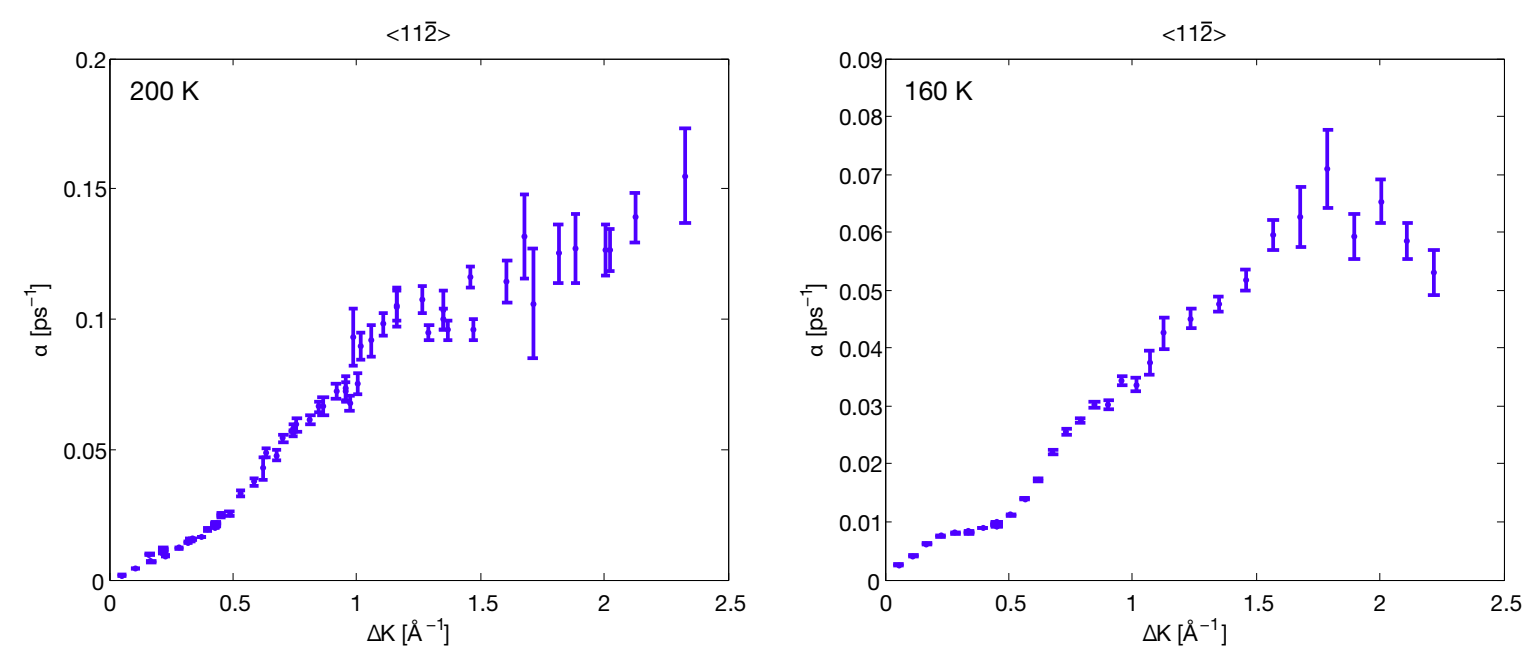

Figure 6.10: $\alpha(\Delta \boldsymbol{K})$ dependence for $0.020 \mathrm{ML}$ pyrrole/ $\mathrm{Cu}(111)$ along $<11 \overline{2}>$ at $200 \mathrm{~K}$ (lefthand side panel) compared to data for the same coverage at $160 \mathrm{~K}$ (right-hand side panel).

$\alpha(\Delta \boldsymbol{K})$ curve for $0.020 \mathrm{ML}$ pyrrole at $200 \mathrm{~K}$ with a curve for the same coverage at $160 \mathrm{~K}$ (reproduced from Figure 6.9). The overall shape of the $\alpha(\Delta \boldsymbol{K})$ curve is very similar at both temperatures, implying that the hopping behaviour does not change significantly with temperature.

Measurements along the two different azimuthal directions, $<1 \overline{1} 0>$ and $<11 \overline{2}>$, show very similar $\alpha(\Delta \boldsymbol{K})$ curves for $0.033 \mathrm{ML}$ pyrrole/Cu(111) at $160 \mathrm{~K}$ (cf. Figure 6.9). Due to the periodicity of the $\mathrm{Cu}(111)$ surface, which is distinctly different along the two azimuths (cf. Figure 2.8), there is no simple analytic jump diffusion model that describes such azimuthally isotropic data [6]. Of the models for jump diffusion presented in Section 2.4.1, the model for hopping on a Bravais lattice, e.g. between top sites, predicts single exponential lineshapes in the ISFs while all models for several sites in the unit cell predict multicomponent lineshapes. The experimental lineshapes for pyrrole/ $\mathrm{Cu}(111)$ (cf. Figures 6.6 and 6.7) are generally well described by a single exponential function along both azimuths, which would typically indicate hopping on a Bravais lattice. However, 
the $\alpha(\Delta \boldsymbol{K})$ dependence along $<11 \overline{2}>$, shown in the right-hand side panel in Figure 6.9, cannot be described by jump diffusion on a simple Bravais lattice, as becomes apparent when comparing the experimental curve with the model curve in Figure 2.9. Most importantly, the analytical curve drops to zero at $2.84 \AA^{-1}$ along $<11 \overline{2}>$, the substrate diffraction position, while the experimental curve shows non-zero decay. It can therefore be concluded that no single process can describe the data and diffusion occurs between several adsorption sites within the unit cell (i.e. a combination of fcc, hcp and/or bridge sites).

The analytical models for jump diffusion on non-Bravais lattices predict a sum of multiple exponential decays in the ISFs, yet such signatures are not observed in the experimental data (cf. Figures 6.6 and 6.7). Note that the small deviations from a single exponential mentioned previously (label C) occur at momentum transfers between $0.2 \AA^{-1}$ and $0.6 \AA^{-1}$ where none of the analytical models predict more than one decay. Section 6.6 will show that the key to reconciling single exponential lineshapes with hopping on multiple sites in the unit cell lies in the strong lateral interactions between the adsorbates.

While it is clear that the analytical models are a simplified description of jump diffusion - e.g. they do not include any information about the lateral inter-adsorbate interactions - it is conceivable that a double decay signature could appear as one single exponential under the noise of the experimental data. In general, the identification of two or several exponentials on similar timescales in experimental ISFs is not trivial [27], as observed in Chapter 5. To rule out potential misinterpretation of the data, the lineshapes predicted by the analytical models were analysed by a single exponential [132] and the resulting $\alpha(\Delta \boldsymbol{K})$ dependence for the single decay compared with the experimental data. Figures 6.11 and 6.12 show the variation of predicted prefactors and dephasing rates with $\Delta \boldsymbol{K}$ as well as the dephasing rate and prefactor for a single exponential along the $<11 \overline{2}>$ direction for hopping on three different non-Bravais lattices: bridge sites; degenerate fcc and hcp sites; and degenerate bridge, fcc and hcp sites.

In all three cases, the single decay is dominated by the predicted slow decay at low $\Delta \boldsymbol{K}$, while the fast decay dominates at higher $\Delta \boldsymbol{K}$, shifting the peak in the $\alpha(\Delta \boldsymbol{K})$ curve from $1.4 \AA^{-1}$ to higher momentum transfers. In the area around the diffraction position $\left(2.84 \AA^{-1}\right)$, the models only predict a single, fast decay, resulting in a discontinuity in the single $\alpha(\Delta \boldsymbol{K})$ curves (cross). The prefactor is a measure of the quality of the fit, illustrating good agreement with a single exponential at small momentum transfers 

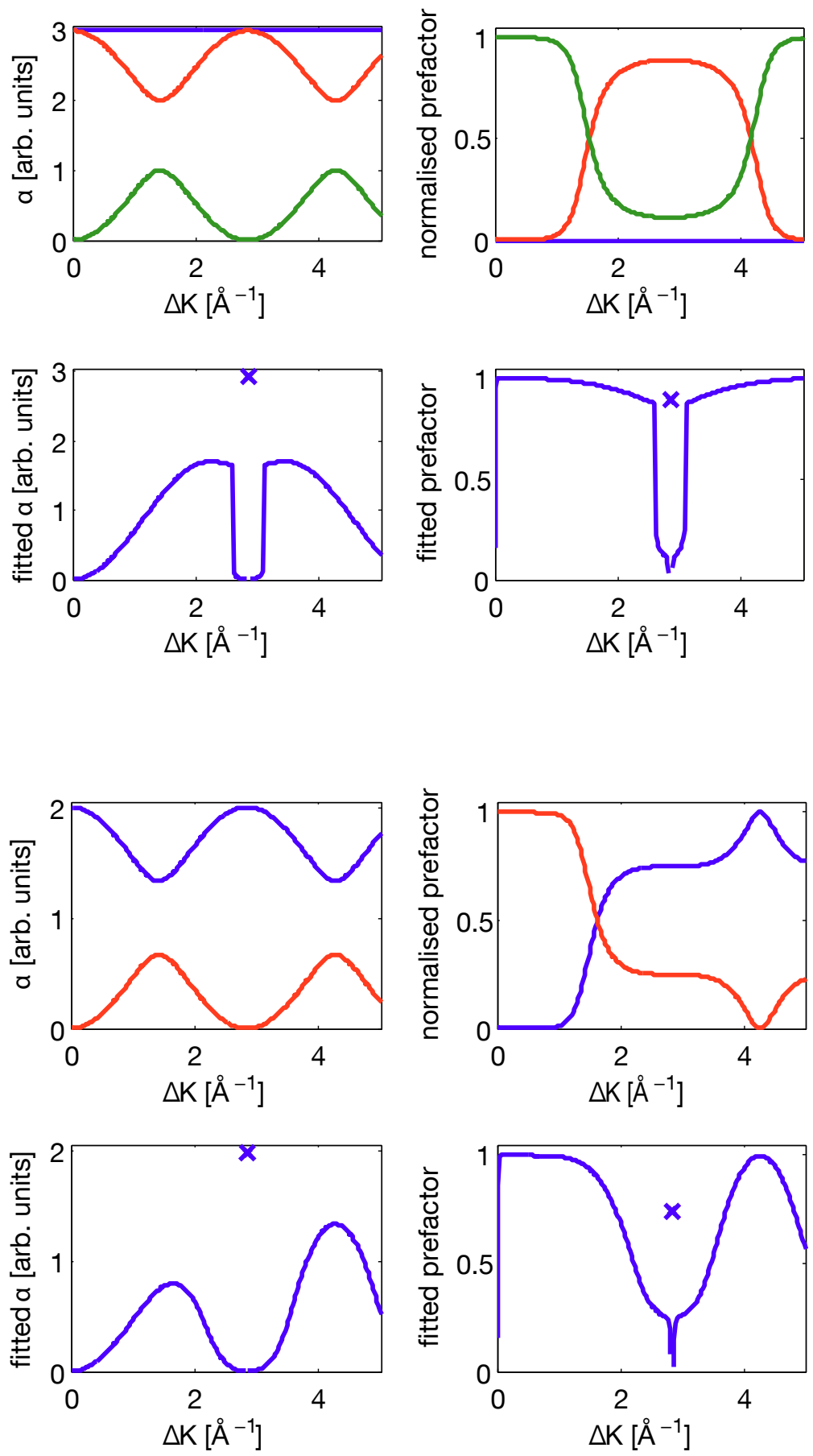

Figure 6.11: $\alpha(\Delta \boldsymbol{K})$ dependence along the $<11 \overline{2}>$ azimuth of a single exponential describing the jump diffusion models for hopping between bridge sites (top four panels) and degenerate fcc and hcp sites (bottom four panels). For each group of four, the top two panels show the predicted decays and pre-exponential factors, respectively, while the bottom panels present a single exponential function analysing the lineshapes. 

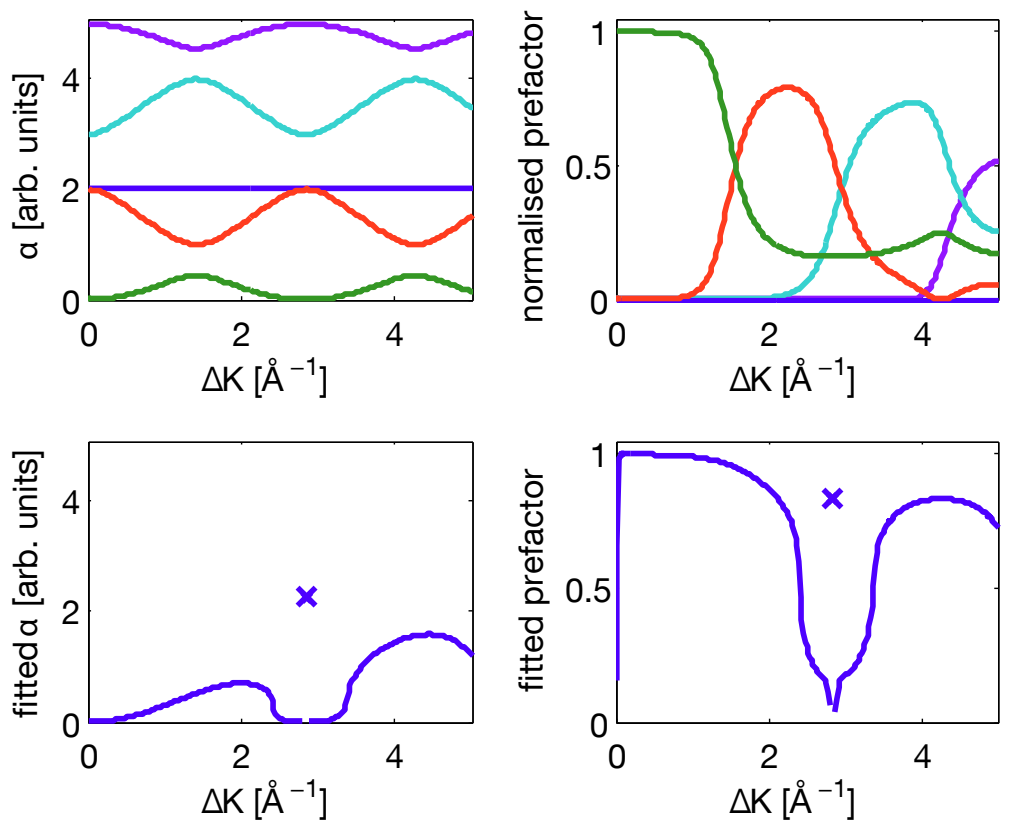

Figure 6.12: $\alpha(\Delta \boldsymbol{K})$ dependence along the $<11 \overline{2}>$ azimuth of a single exponential describing the jump diffusion models for hopping between degenerate bridge, fcc and hcp sites. The top two panels show the predicted decays and pre-exponential factors, respectively, while the bottom panels present a single exponential function analysing the lineshapes.

$\left(<1 \AA^{-1}\right)$ where the models predict most of the intensity for only one decay and a clear deviation from a single exponential in the diffraction point area. These findings confirm that the experimental data for pyrrole $/ \mathrm{Cu}(111)$ cannot be explained by simple analytical models. It is therefore necessary to apply a different, more complete, analysis method that includes lateral inter-adsorbate interactions, such as molecular dynamics simulations.

Summarising the principle experimental observations, temperature dependent measurements indicate that pyrrole diffusion is a strongly activated process. The uptake behaviour as well as the de Gennes feature in the $\alpha(\Delta \boldsymbol{K})$ curves reveal the presence of strong lateral interactions that influence the diffusive motion. At high $\Delta \boldsymbol{K}$, similar behaviour is observed along the $<1 \overline{1} 0>$ and $<11 \overline{2}>$ azimuths. The shape of the $\alpha(\Delta \boldsymbol{K})$ curves implies that pyrrole hops between several adsorption sites in the unit cell, yet the lineshapes in the experimental ISFs appear as a single exponential. Investigating the lineshapes predicted by analytical models, it becomes obvious that the experimental data cannot be explained by simple analytical models that do not include adsorbate-adsorbate 
interactions. Therefore, the need for molecular dynamics simulations arises, where the effect of repulsive lateral interactions can be modelled, as described in Section 6.6.

\subsection{First principles density functional theory calculations}

As for $\mathrm{Cp} / \mathrm{Cu}(111)$, Sacchi and Jenkins from the Department of Chemistry at Cambridge have performed first principles density functional theory (DFT) calculations to provide further information on the adsorption behaviour of pyrrole on $\mathrm{Cu}(111)$. This section summarises their results, which are crucial to the interpretation of the HeSE measurements.

Pyrrole/ $\mathrm{Cu}(111)$ is a physisorbed system, bonding predominantly through weak dispersion forces. Therefore, van der Waals corrections need to be included in the calculations. Sacchi and Jenkins carried out their calculations using CASTEP [103], employing the dispersion force correction method by Tkatchenko and Scheffler [133] calculated with the Perdew-Burke-Ernzerhof (PBE) functional. At sub-monolayer coverages, pyrrole is found to adsorb in a flat-lying geometry on $\mathrm{Cu}(111)$, in agreement with experimental observations for adsorption on $\mathrm{Cu}(100)$ [121] and other metal surfaces (cf. Figure 6.2) [123,124,128,129]. Calculations show that the molecule adsorbs centrally on high symmetry sites. In addition, the electron density obtained from calculations can be used to predict contours of the adsorbate in a beam using the effective-medium theory by Nørskov et al. [134,135]. Surface potential, $V$, and isocharge surface, $\rho$, are related through a simple proportionality factor $V / \rho=45 \mathrm{eV} \AA^{3}$ which can be used to select the contour map for the desired beam energy $[135,136]$. By determining charge density contours corresponding to a beam energy similar to that used in HeSE experiments, Sacchi and Jenkins have demonstrated that the nitrogen atom does not appear significantly different from the carbon atoms in a helium beam. Therefore, pyrrole has cylindrical symmetry in helium diffraction experiments.

Calculated adsorption energies for different high symmetry sites and molecular orientations are summarised in Figure 6.13 for two coverages, $(\sqrt{7} \times \sqrt{7})$ R19.1 $1^{\circ}$ corresponding to $0.14 \mathrm{ML}$ and $(2 \sqrt{3} \times 2 \sqrt{3}) \mathrm{R} 30^{\circ}$ corresponding to $0.08 \mathrm{ML}$ [95]. At both coverages, the preferred adsorption site is a bridge site (bottom centre), with the least favourable site on top, $126 \mathrm{meV}$ above the bridge sites. Both fcc and hcp hollow sites are within $15 \mathrm{meV}$ 
of the bridge site, for the respective favoured rotational orientation of the molecule. In addition to the geometries shown, calculations of positions intermediate between bridge and hollow sites show an energy barrier of less than $5 \mathrm{meV}$. Equally, no significant barrier to rotation is found.

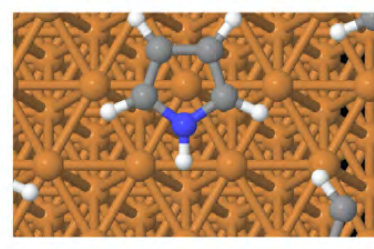

$-0.896,-0.932$

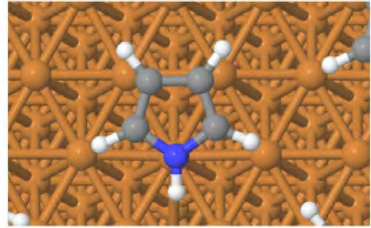

$-0.988,-1.043$

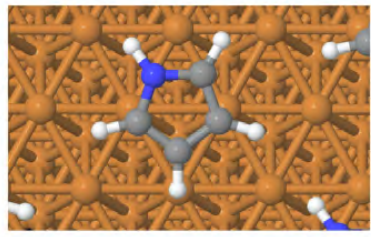

$-1.007,-1.039$

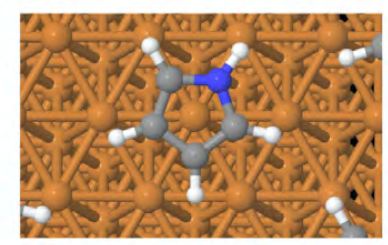

$-0.8906,-0.915$

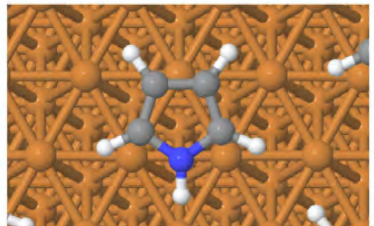

$-0.993,-1.043$

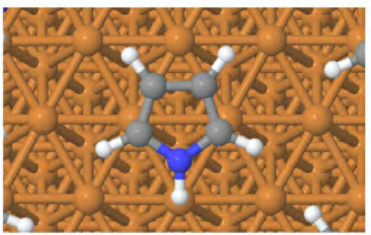

$-1.015,-1.058$

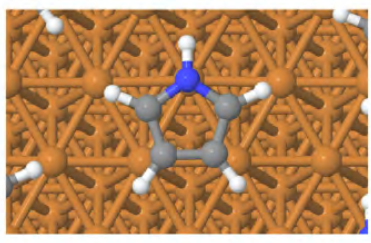

$-0.976,-1.007$

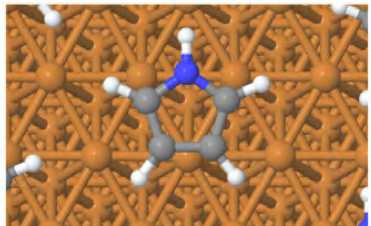

$-1.000,-1.023$

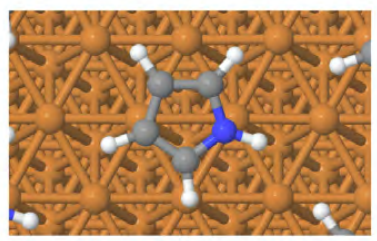

$-0.987,-1.040$

Figure 6.13: Illustration by Sacchi and Jenkins, Cambridge [95], summarising the adsorption energies (in eV) for pyrrole/ $\mathrm{Cu}(111)$ calculated using first principles DFT. Values for two different coverages are shown: $(\sqrt{7} \times \sqrt{7})$ R19.1 $1^{\circ}$ (black numbers) and $(2 \sqrt{3} \times 2 \sqrt{3}) \mathrm{R} 30^{\circ}$ (red numbers).

These results suggest that pyrrole can move freely in a channel of bridge and hollow sites around top sites, thus confirming the experimental observation that pyrrole jumps between several sites in the unit cell. The calculated energy barriers, however, are significantly smaller than the measured apparent Arrhenius barrier of $53 \pm 4 \mathrm{meV}$. Section 6.7 shows that this seeming discrepancy between experiment and theory is due to site-dependent differences in the zero point energies which are not included in the present calculations.

In addition, first principles calculations give the work function change and dipole moment at two different coverages, summarised in Table 6.3. From these results, the dipole moment in the low coverage limit, $p_{0}=6.17$ Debye, and the polarisability, 
$\alpha_{p}=152 \AA^{3}$, are obtained by applying the Topping model (cf. Chapter 5), which can be used to define the interactions in molecular dynamics simulations [105].

\begin{tabular}{cccc}
\hline structure & $\boldsymbol{\Theta}[\mathrm{ML}]$ & $\boldsymbol{\Delta} \boldsymbol{\Phi}[\mathrm{eV}]$ & $\boldsymbol{p}[$ Debye $]$ \\
\hline$(2 \sqrt{3} \times 2 \sqrt{3}) \mathrm{R} 30^{\circ}$ & 0.08 & -0.996 & 1.80 \\
$(\sqrt{7} \mathrm{x} \sqrt{7}) \mathrm{R} 19.1^{\circ}$ & 0.14 & -0.906 & 0.96 \\
\hline
\end{tabular}

Table 6.3: Work function change and dipole moment of pyrrole/Cu(111) from DFT calculations by Sacchi and Jenkins [95].

\subsection{Molecular dynamics simulations investigate the effect of lateral interactions}

Experimental data presented in Section 6.4.3 suggests that pyrrole hops between multiple sites in the unit cell. The lineshapes in the experimental ISFs cannot be described by a simple analytical model, suggesting that an alternative analysis method should be employed. Martínez-Casado et al. have suggested previously that lateral interactions between adsorbates alter the lineshapes [34]. To investigate this effect, molecular dynamics (MD) simulations are performed to get a more complete picture of the motion.

The general principle of classical 2D Langevin molecular dynamics simulations is described in Section 2.4.3, modelling adsorbate motion over a potential energy surface (PES), $V\left(\boldsymbol{r}_{i}\right)$ (cf. Equation 2.26) [42]. The adsorbate-substrate interactions are represented by the friction term, $\eta$, and a random impulse term, $\xi$, ensures energy conservation, while inter-adsorbate interactions are described by pairwise forces, $F_{i, j}$. In these simulations pyrrole is treated as a single point particle of mass $67 \mathrm{amu}$. To reproduce the shape of the experimental ISFs as well as their momentum transfer and temperature dependence, the friction coefficient, $\eta$, the barrier height, the shape of the adsorption well and the interaction forces, $F_{i, j}$, are optimised iteratively. Pair-wise interactions are defined using the zero coverage limit dipole moment and polarisability calculated from DFT results (cf. Section 6.5). Simulations without interaction forces were run for a single adsorbate particle, while simulations including pair-wise interactions include 40 particles at coverages corresponding to those used in experiment: 0.020, 0.033 and 0.052 ML. 
At the start of a simulation, each of 40 randomly allocated adsorbate particles is given a random kinetic energy in all directions and the system is allowed to equilibrate for 2000 steps before the actual simulation starts. The intermediate scattering function is calculated from the trajectories in $0.16 \mathrm{ps}$ time steps. For each point in the $\alpha(\Delta \boldsymbol{K})$ curve a total of 32 runs, each 480 ps long, are averaged and the resulting ISFs analysed using an exponential function, treating it in the same way as the experimental data.

\subsubsection{Creating a potential energy surface}

In order to simulate the diffusion behaviour of pyrrole/ $\mathrm{Cu}(111)$, a PES is required that represents the periodicity of different combinations of adsorption sites on $\mathrm{Cu}(111)$ and allows optimisation of the shape of the adsorption well and the energy barrier for hopping motion. The method employed here follows a procedure similar to that presented in Section $5.6[43,44]$. The potential is defined as a Fourier series,

$$
V(\boldsymbol{r})=-\sum_{i, n} A_{n} \cos \left(n \boldsymbol{g}_{i} \cdot \boldsymbol{r}\right)
$$

where three vectors $\boldsymbol{g}_{i}$ define the geometry of the hexagonal $\mathrm{Cu}(111)$ substrate

$$
\begin{array}{r}
\boldsymbol{g}_{1}=(\zeta, 0), \\
\boldsymbol{g}_{2}=(\zeta \cos (\pi / 3), \zeta \sin (\pi / 3)), \\
\boldsymbol{g}_{3}=(-\zeta \cos (\pi / 3), \zeta \sin (\pi / 3))
\end{array}
$$

$\zeta$ is related to the $\mathrm{Cu}(111)$ lattice constant, $a$, through $\zeta=4 \pi /(\sqrt{3} a)$. Using different combinations of first $(n=1)$ and third Fourier coefficients $(n=2)$, the energy for top, bridge and hollow sites can be selected. ${ }^{2}$

Several example PES are shown in Figure 6.14 that have been optimised to reproduce the pyrrole/ $\mathrm{Cu}(111)$ dynamics as best possible within the given jump diffusion model. In the simplest form, only the first Fourier coefficient is used $(n=1)$, which produces a PES for top site adsorption with the transition state on the bridge sites (cf. Figure $6.14(\mathrm{~A})$ ). Inverting this potential by making $A_{1}$ negative, still keeping the first Fourier components only, results in a PES for hollow site adsorption, with both fcc and hcp sites set to zero

\footnotetext{
${ }^{2}$ This simple model only allows for energetically degenerate hollow sites. If required, non-degeneracy
} could be created by adding an imaginary part of the first Fourier coefficient. 

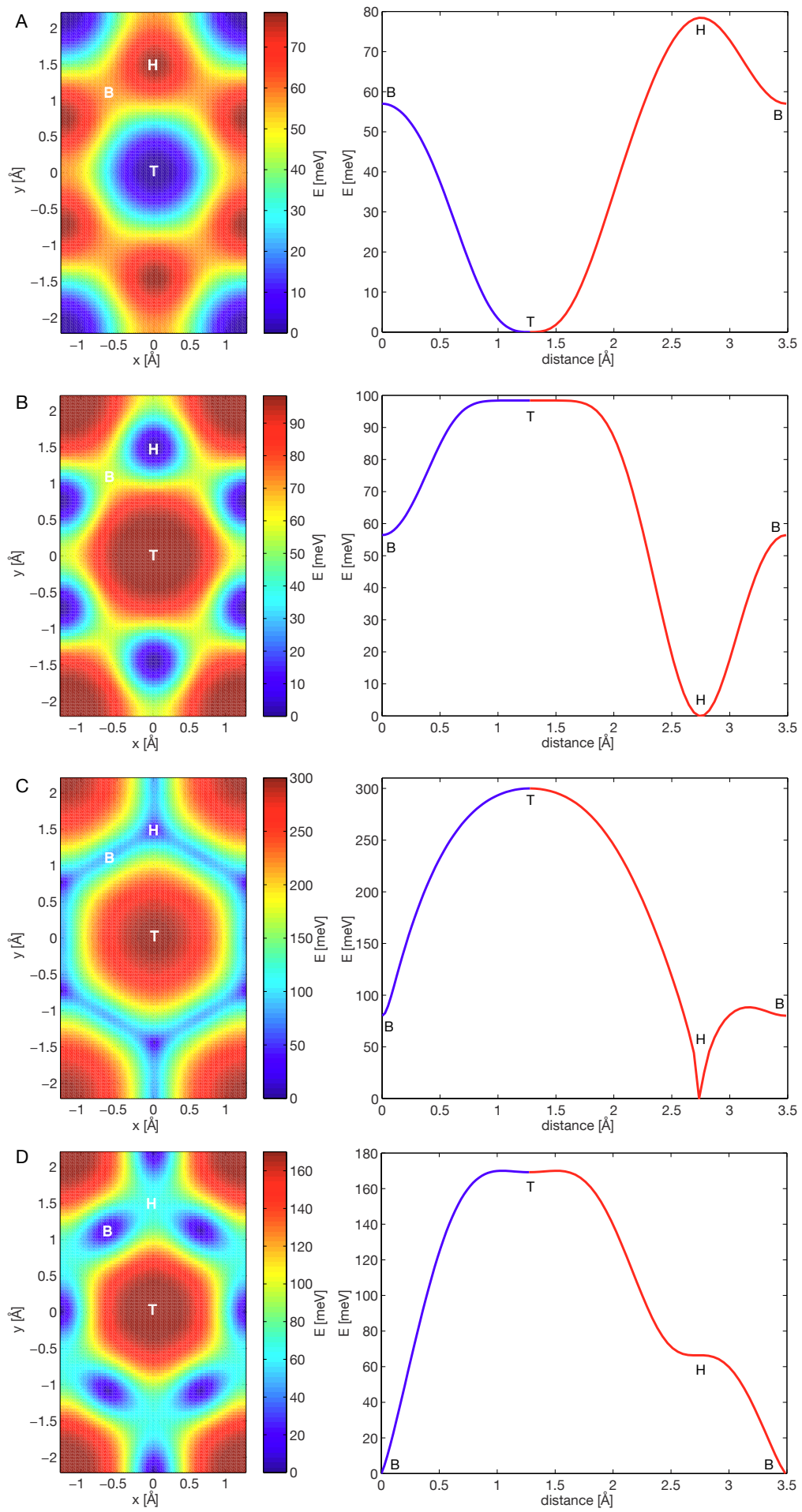

Figure 6.14: PES to model the diffusion of pyrrole $/ \mathrm{Cu}(111)$ between (A) top sites, (B) hollow sites, (C) hollow and bridge sites and (D) bridge sites. The left-hand side panels show a top view of the respective PES, while the right-hand side panels represent a cross section from bridge to top site along $<1 \overline{1} 0>$ (blue) and from top to hollow and bridge site along $<11 \overline{2}>$ (red). All examples shown are optimised to describe the pyrrole/ $\mathrm{Cu}(111) \mathrm{HeSE}$ data as best possible within the respective models. The $p$ values used here are (A) 1.5, (B) 0.25, (C) 0.3 and (D) 0.6. 
and the transition state remaining on bridge sites (cf. Figure 6.14 (B)), just like the version used in Chapter 5.

Adding the second Fourier component can be used to create additional sites. In reference [44], $A_{1}$ was kept positive to create minima at the top site positions and additional minima were added on bridge sites using the second Fourier component $\left(A_{2}>0.25 A_{1}\right)$. Here, $A_{1}$ is kept negative (hollow site adsorption) and the second Fourier components are added to obtain a PES for combined hollow and bridge site adsorption. Figure $6.14(\mathrm{C})$ shows an example for such a combined PES, resulting in a channel around the top sites with a barrier in between bridge, fcc and hcp sites. The third Fourier coefficient creates dips at hollow and bridge sites, while the first one contributes only to hollow sites. Therefore, by subtracting the first from the third Fourier components a PES for adsorption on bridge sites alone is obtained, with the transition state over hollow sites (cf. Figure $6.14(\mathrm{D})$ ).

In addition to defining the relative energy of the different high symmetry sites, the shape of the PES can be altered by first scaling the PES ranging from 0 to $E_{\max }$ to give 0 to 1 on the energy scale, then raising it to a power, $p$, to change the curvature of the PES without changing the absolute energy range and finally scaling it back to the original height, $E_{\max }$. Changing $p$ allows flattening or sharpening of the wells at the preferred adsorption sites, making the transition state narrower or wider, respectively. The $p$ values used in Figure 6.14 are (A) 1.5, (B) 0.25, (C) 0.30 and (D) 0.60, respectively. Note that the shape of the peak (least preferable adsorption site) does not influence the motion, as can be confirmed by the trajectories shown in Figure 6.19.

\subsubsection{Modelling the diffusion of pyrrole/Cu(111)}

MD simulations were performed with a variety of energy landscapes, each time optimising the friction, the energy barrier and the shape of the adsorption well to best reproduce the experimental results in terms of momentum transfer and temperature dependence. The best result within each jump diffusion model is compared with the experimental results to determine the preferred adsorption sites. Single particle simulations on the same PES allow the investigation of the effect of lateral interactions on the lineshapes in the ISFs, suggested by Martínez-Casado et al. [34].

While top sites have been ruled out as preferred adsorption sites by DFT calculations and the analytical models, simulations modelling the diffusion on a simple Bravais lattice 


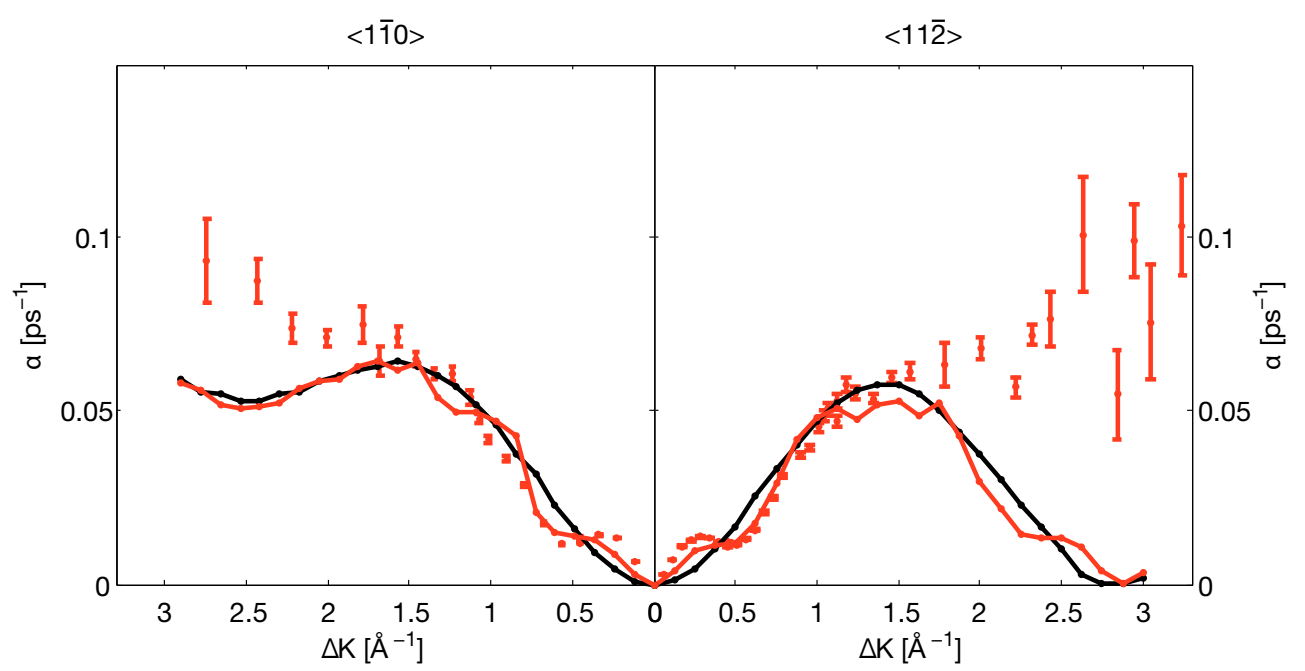

Figure 6.15: MD simulations for $0.033 \mathrm{ML}$ pyrrole $/ \mathrm{Cu}(111)$ at $160 \mathrm{~K}$, jumping between top sites, compared to the experimental data (dots). A single particle simulation is presented as a black line and a simulation including repulsive interactions as a red line.

were performed to confirm these results when lateral interactions are included, comparing the simulated $\alpha(\Delta \boldsymbol{K})$ dependence for a single particle with that of 40 repulsively interacting adsorbates. Figure 6.15 compares the experimental data at 0.033 ML with the curves from simulations, where a friction value of $\eta=2.8 \pm 0.2 \mathrm{ps}^{-1}$ and an activation barrier of $57 \pm 2 \mathrm{meV}$ was employed (cf. Figure 6.14 (A)). As predicted by analytical models, the dephasing rate peaks at $1.4 \AA^{-1}$ and drops to zero at the diffraction position of $2.84 \AA^{-1}$ along $<11 \overline{2}>$, for the single particle simulations (black) as well as for that with interactions (red). Repulsive forces result in strong de Gennes narrowing, apparent as a peak and dip at low momentum transfers and mirrored from the diffraction position and weak second order features occur at twice the value in momentum transfer. It can thus be concluded that the interactions have a strong effect on the momentum transfer dependence of the dephasing rate, yet the earlier conclusion that pyrrole does not hop on a Bravais lattice still stands despite lateral forces.

Next, MD simulations investigate a range of different potential energy surfaces for non-Bravais lattices in a channel around the top sites, as suggested by DFT calculations. Specifically, three different PES are studied: one for hopping between degenerate fcc and hcp hollow sites over an energy barrier of $56 \pm 5 \mathrm{meV}$, a combined PES for jump diffusion between bridge and hollow sites that exhibits a barrier of $80 \pm 6 \mathrm{meV}$, and one for jumps between bridge sites over a barrier of $66 \pm 3 \mathrm{meV}$ (cf. Figure 6.14 (B), (C) and (D)). Figures $6.16,6.17$ and 6.18 show the HeSE data at three different coverages compared 


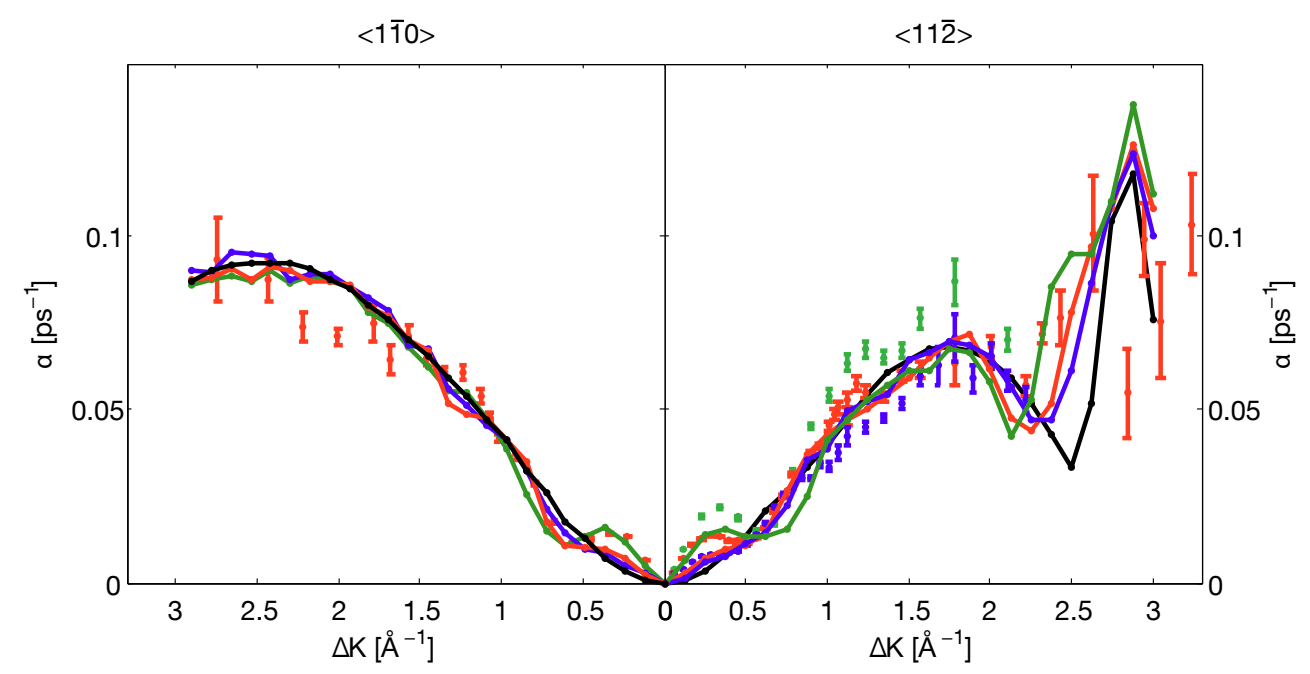

Figure 6.16: MD simulations (lines) for $0.020 \mathrm{ML}$ (blue), 0.033 ML (red) and 0.052 ML (green) pyrrole/ $\mathrm{Cu}(111)$ at $160 \mathrm{~K}$ jumping between degenerate fcc and hcp sites, compared to the experimental data in the respective colours (dots). Single particle simulation data are shown as a black line.

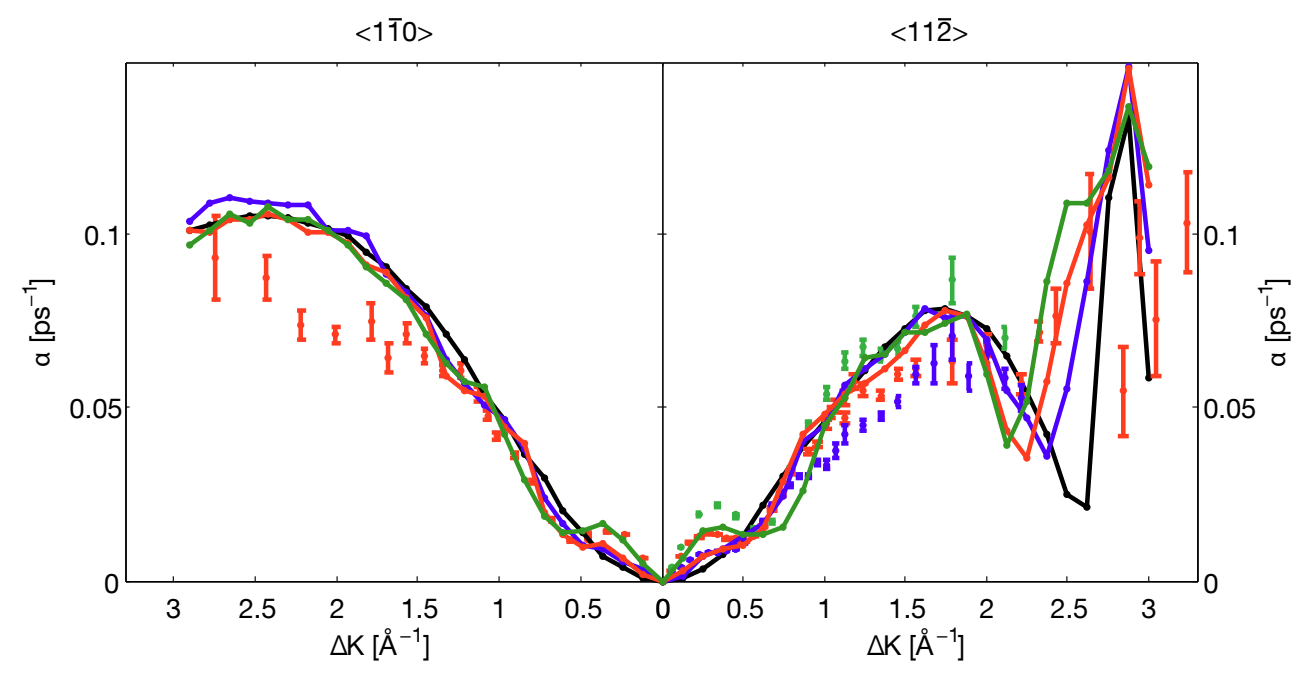

Figure 6.17: MD simulations (lines) for 0.020 ML (blue), 0.033 ML (red) and 0.052 ML (green) pyrrole/ $\mathrm{Cu}(111)$ at $160 \mathrm{~K}$ jumping between bridge and hollow sites, compared to the experimental data in the respective colours (dots). Single particle simulation data are shown as a black line.

to each of the simulations, respectively. All simulated ISFs are described by a single exponential function to give a realistic comparison between experiment and simulation.

Overall, all three simulations describe the data reasonably well. The similarity between the three models shown in Figures $6.16,6.17$ and 6.18 can be understood by comparing 


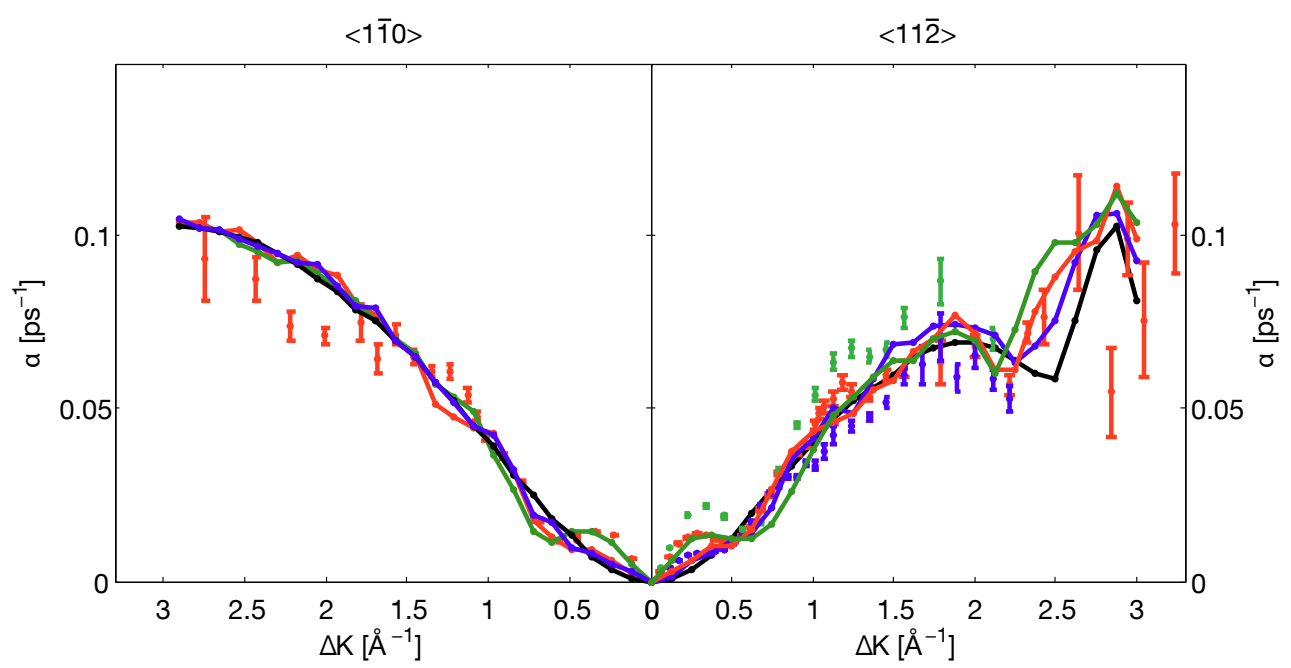

Figure 6.18: MD simulations (lines) for $0.020 \mathrm{ML}$ (blue), $0.033 \mathrm{ML}$ (red) and $0.052 \mathrm{ML}$ (green) pyrrole/ $\mathrm{Cu}(111)$ at $160 \mathrm{~K}$ jumping between bridge sites, compared to the experimental data in the respective colours (dots). Single particle simulation data are shown as a black line.

the adsorbate trajectories from MD simulations, shown in Figure 6.19. Hopping on top sites gives a distinct pattern of particle positions within adsorption wells, while the other three models all look very similar, showing motion in channels of hollow and bridge sites around top sites with little difference between the three models. Therefore, it is not surprising that the latter three models result in similar $\alpha(\Delta \boldsymbol{K})$ curves along both azimuths. While they all give a good description, however, the simulation for hopping between bridge sites best reproduces the experimental shape (cf. Figure 6.18), suggesting that pyrrole adsorbs preferably on bridge sites.

All MD simulations are optimised to create the same temperature dependent behaviour as observed in the experiments. The apparent Arrhenius barrier for all simulated results presented in this section are on the order of $50 \pm 2 \mathrm{meV}$, i.e. within error bars of the experimental barriers. Figure 6.20 shows a comparison between experimental data at $200 \mathrm{~K}$ and the simulation for jumps between bridge sites at the same temperature. Just like at $160 \mathrm{~K}$, good overall agreement is found.

All three models for motion in channels around top sites give a friction coefficient of $\eta=2.0 \pm 0.4 \mathrm{ps}^{-1}$. As discussed in Section 5.6, this is a high friction compared to smaller adsorbate species and similar to that found for $\mathrm{Cp} / \mathrm{Cu}(111)$ (cf. Chapter 5). The friction coefficient is in good agreement with the timescales on which the signature for intracell diffusion is observed in the experimental ISFs, shown in Figures 6.6 and 6.7, in 

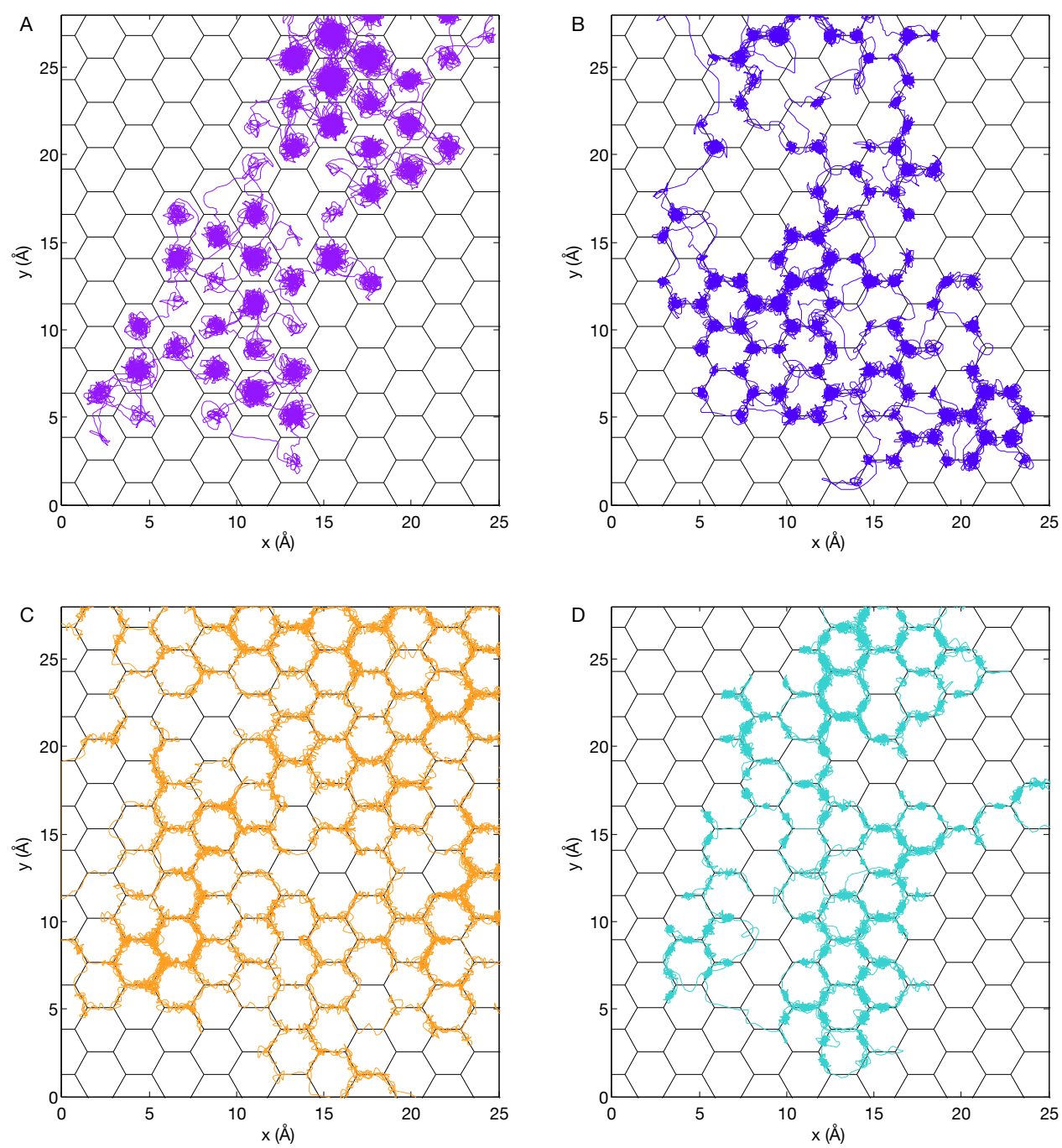

Figure 6.19: Trajectories for MD simulations on a PES for adsorption on (A) top sites, (B) fcc and hcp sites, (C) fcc, hcp and bridge sites and (D) bridge sites. The trajectories for B, C and D look qualitatively similar, while A shows significantly different behaviour.

good agreement with the results for $\mathrm{Cp} / \mathrm{Cu}(111)$ (cf. Section 5.9). From the friction, the proportion of double jumps can be calculated as $\exp (-t \eta)=8 \%$ (cf. Section 5.6). The adsorbate molecules thus move predominantly in single jumps between adjacent bridge sites.

Including pair-wise forces in the simulations much improves the shape of the $\alpha(\Delta \boldsymbol{K})$ curves, in particular along $<11 \overline{2}>$. Firstly, de Gennes narrowing becomes apparent, increasing in intensity and moving to larger momentum transfers as the coverage is increased. Secondly, the dip at $\sim 2.5 \AA^{-1}$ that appears in the single particle simulations 


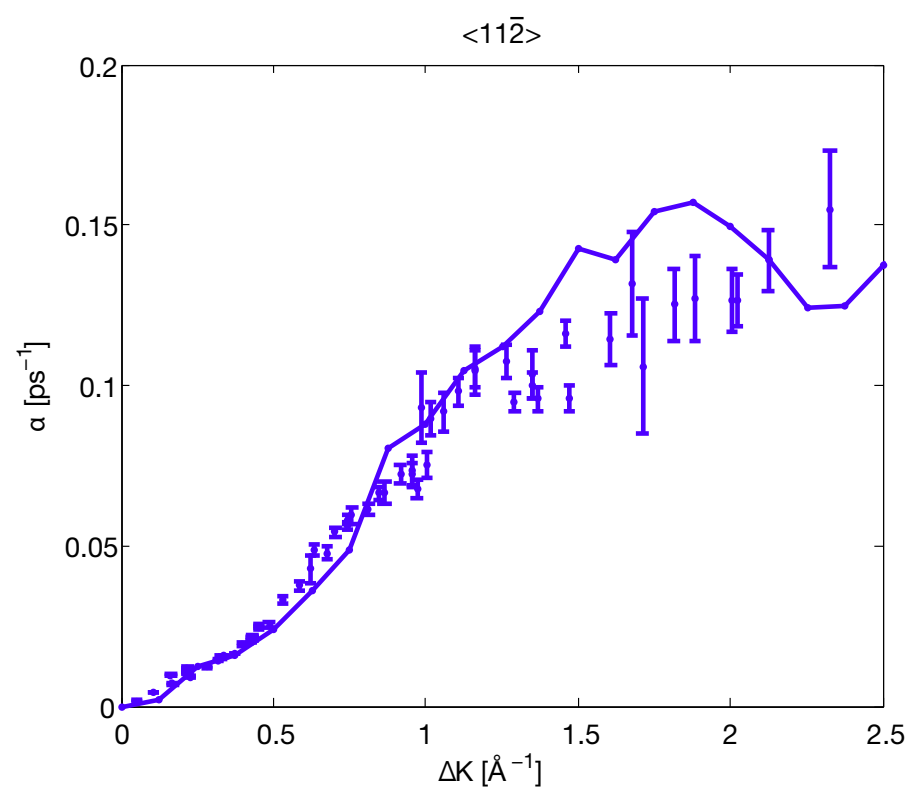

Figure 6.20: MD simulations (line) for $0.020 \mathrm{ML}$ pyrrole/Cu(111) at $200 \mathrm{~K}$ jumping between bridge sites, compared to the experimental data (dots).

(black lines) becomes less pronounced and moves to smaller momentum transfers, thus improving the quality of the simulation significantly. Note that while the de Gennes features are symmetric around the diffraction position for hopping on simple Bravais lattices (as observed in the MD simulations for jump diffusion between top sites, cf. Figure 6.15), the same is not true for non-Bravais lattices as multiple components are expected in the lineshapes of the ISFs. The following sections discuss the effects of lateral interactions in the low and high momentum transfer regions separately.

\subsubsection{Interaction potential}

The position of the de Gennes narrowing is in good agreement with the experimental dip and moves to higher momentum transfers with increasing coverage, confirming the coverage determination from HeSE data. The magnitude of the peak, however, is somewhat smaller than in the experiment, suggesting that the interactions are not perfectly reproduced in the simulations. In order to rule out inaccuracies in the DFT calculations, an attempt at altering the dipole interaction strength in the simulations was made. However, in order to reproduce the magnitude of the peak observed in experiment, the dipole moment needs to be increased sufficiently that a second order de Gennes narrowing at approximately $1.2 \AA^{-1}$ is created which is not in agreement 
with the experimental $\alpha(\Delta \boldsymbol{K})$ curves. The creation of second order features implies that the long-distance behaviour of a pair-wise dipole potential does not correctly describe pyrrole-pyrrole interactions.

In order to model the interactions more accurately, a new potential was created by distributing the total dipole moment equally in five dipoles located near the carbon and nitrogen atoms, thus adding an effect of repulsion when two molecules would start to overlap, which is not included in the standard potential for point-like dipoles. Figure 6.21 shows a comparison of the standard $1 / r^{3}$ dipole-dipole potential and a potential for five partial dipoles, each with a fifth of the dipole strength calculated with DFT.

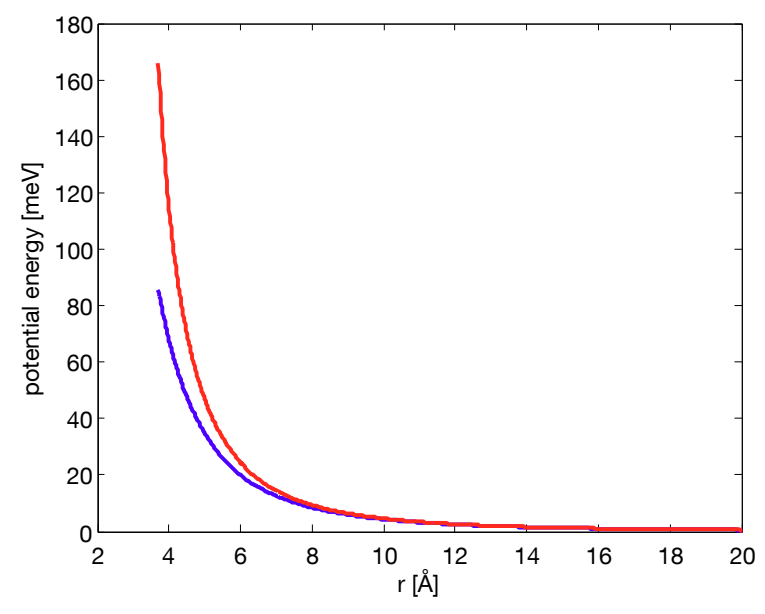

Figure 6.21: Comparison of a standard $1 / r^{3}$ dipole-dipole potential (blue) and the potential for five partial dipoles located near the carbon and nitrogen atoms, each with a fifth of the dipole strength predicted by DFT simulations (red).

At distances larger than $8 \AA$, the new potential is similar to the dipole-dipole potential, but it rises more steeply as the two molecules approach each other (the distance $r$ is defined between the geometric centres of the molecules). A higher order potential thus needs to be added to the dipole-dipole field. Fitting a function of the form $f(r)=a\left(\frac{1}{r^{3}}+\frac{b}{r^{c}}\right)$ to the new potential gives $a \approx 1, b \approx 130$ and $c \approx 7$, suggesting that a $1 / r^{7}$ potential needs to be added in the MD simulations. The power of 7 is found to be essentially independent of the precise location of the partial dipoles.

Repeating the MD simulations for hopping between bridge sites using the new potential shows an increase in the intensity of the higher order features compared to the results for a simple dipole-dipole interaction potential. The new interaction potential thus describes the experimental results less accurately than the $1 / r^{3}$ potential. While first order de Gennes features are a result of the local interactions between nearest neighbour 
adsorbates and are thus directly controlled by the interaction strength, second order features show a more complex behaviour. Although long-range interactions are too weak themselves to force long-range ordering, stronger short-range interactions create better local ordering which in turn forces long-range order.

It can therefore be concluded that the interaction potential for pyrrole $/ \mathrm{Cu}(111)$ is of a more complex nature. In order to study this effect further, a more detailed theoretical study would be needed. For the purpose of investigating the dynamics of pyrrole $/ \mathrm{Cu}(111)$, however, the $1 / r^{3}$ potential represents a sufficiently accurate approximation since the interaction potential influences data most at low $\Delta \boldsymbol{K}$ values, while the main focus here is on the shape of the $\alpha(\Delta \boldsymbol{K})$ curve at larger momentum transfers.

\subsubsection{Influences of lateral interactions on the lineshapes}

The second effect of including lateral interactions in MD simulations, is a change in the shape of the $\alpha(\Delta \boldsymbol{K})$ curve at high momentum transfers. In order to study the influence of lateral interactions on the lineshapes predicted by Martínez-Casado et al. [34], ISFs for single and multiple particle MD simulations are compared. Figures 6.22 and 6.23 show a series of simulated lineshapes at different momentum transfer values, along the $<1 \overline{1} 0>$ and $<11 \overline{2}>$ azimuths, respectively, obtained from simulations modelling jump diffusion between bridge sites at a coverage of $0.033 \mathrm{ML}$. Blue lines show the lineshapes for single particle simulations, while red lines are for simulations including lateral interactions. In addition, the residual after subtracting a single exponential from either lineshape is shown in green (single particle simulation) and cyan (multiple particle simulation).

At low momentum transfer values, lateral interactions result in a clear change in the lineshapes along both azimuths. The residual after subtracting a single exponential from the ISFs for simulations including repulsive forces deviates from zero more than the residual for single particle simulations, implying that lateral interactions change the lineshapes from a single exponential to a more complex form. This effect is found for $\Delta \boldsymbol{K}<0.8 \AA^{-1}$, which is where the de Gennes feature occurs in the $\alpha(\Delta \boldsymbol{K})$ curves (cf. Figure 6.9) and where small deviations from a single exponential are observed in the experimental lineshapes (cf. Figures 6.6 and 6.7).

Along $<1 \overline{1} 0>$, the lineshapes at higher momentum transfers remain essentially unchanged when lateral interactions are included (cf. Figure 6.22). Along $<11 \overline{2}>$, however, lateral interactions also alter the lineshapes at high momentum transfers $\left(>2.0 \AA^{-1}\right)$. 

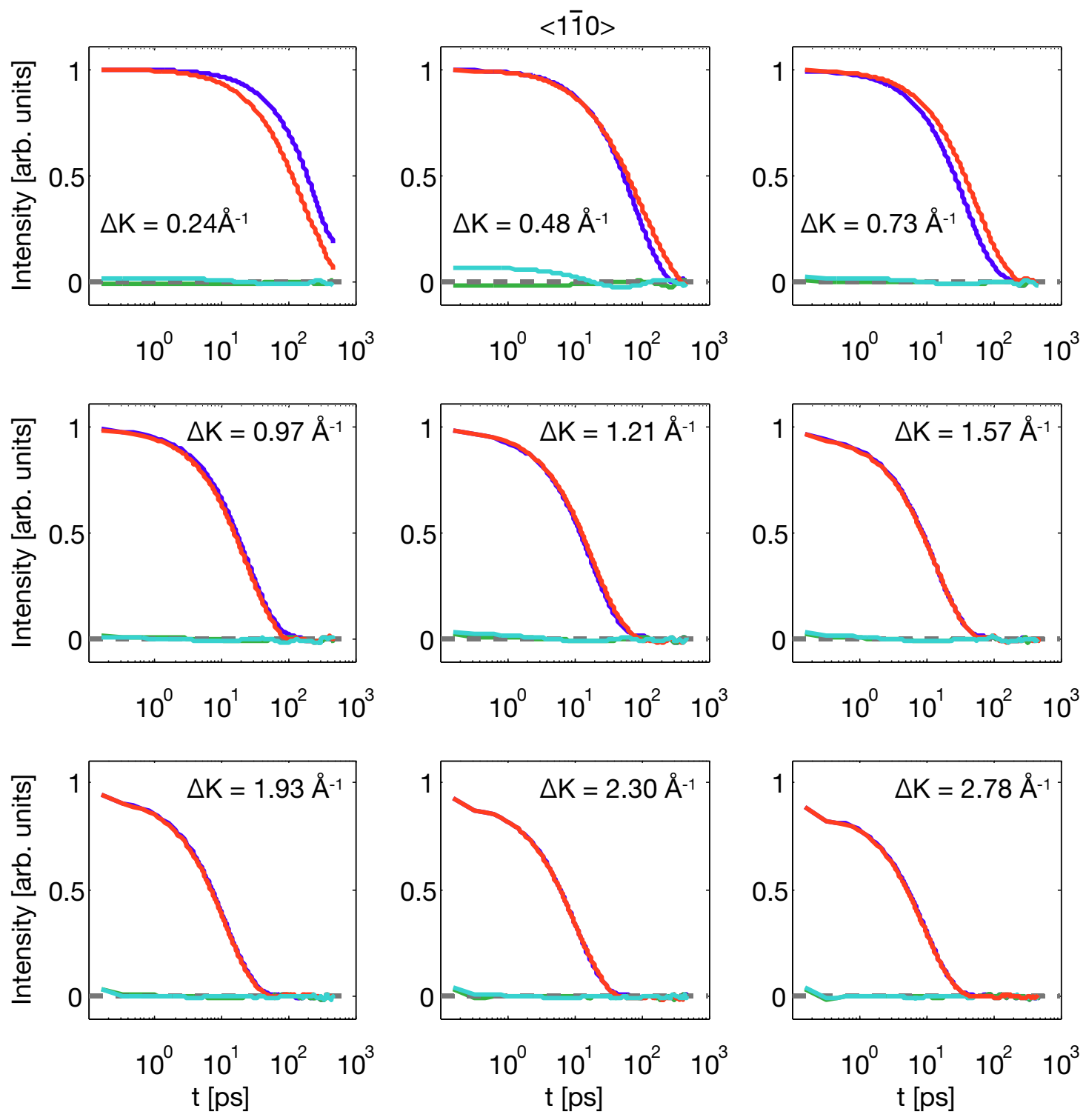

Figure 6.22: Polarisation curves as a function of spin-echo time for MD simulations of $0.033 \mathrm{ML}$ of pyrrole $/ \mathrm{Cu}(111)$ along $<1 \overline{1} 0>$ without and with pair-wise interactions. The different panels show results at increasing momentum transfers. ISFs from single particle simulations are shown as blue lines, those from simulations including pair-wise interactions as red lines. The residuals after subtracting a single exponential of the functional form $f(t)=a \cdot \exp (-\alpha t)+c$ from either ISFs are shown in green and cyan for the single and multiple particle simulations, respectively.

While the residual shows a clear deviation from zero for single particle simulations, the deviation is generally smaller (i.e. the amplitude in the residual is lower) when pair-wise lateral interactions are included, implying that inter-adsorbate interactions cause the 

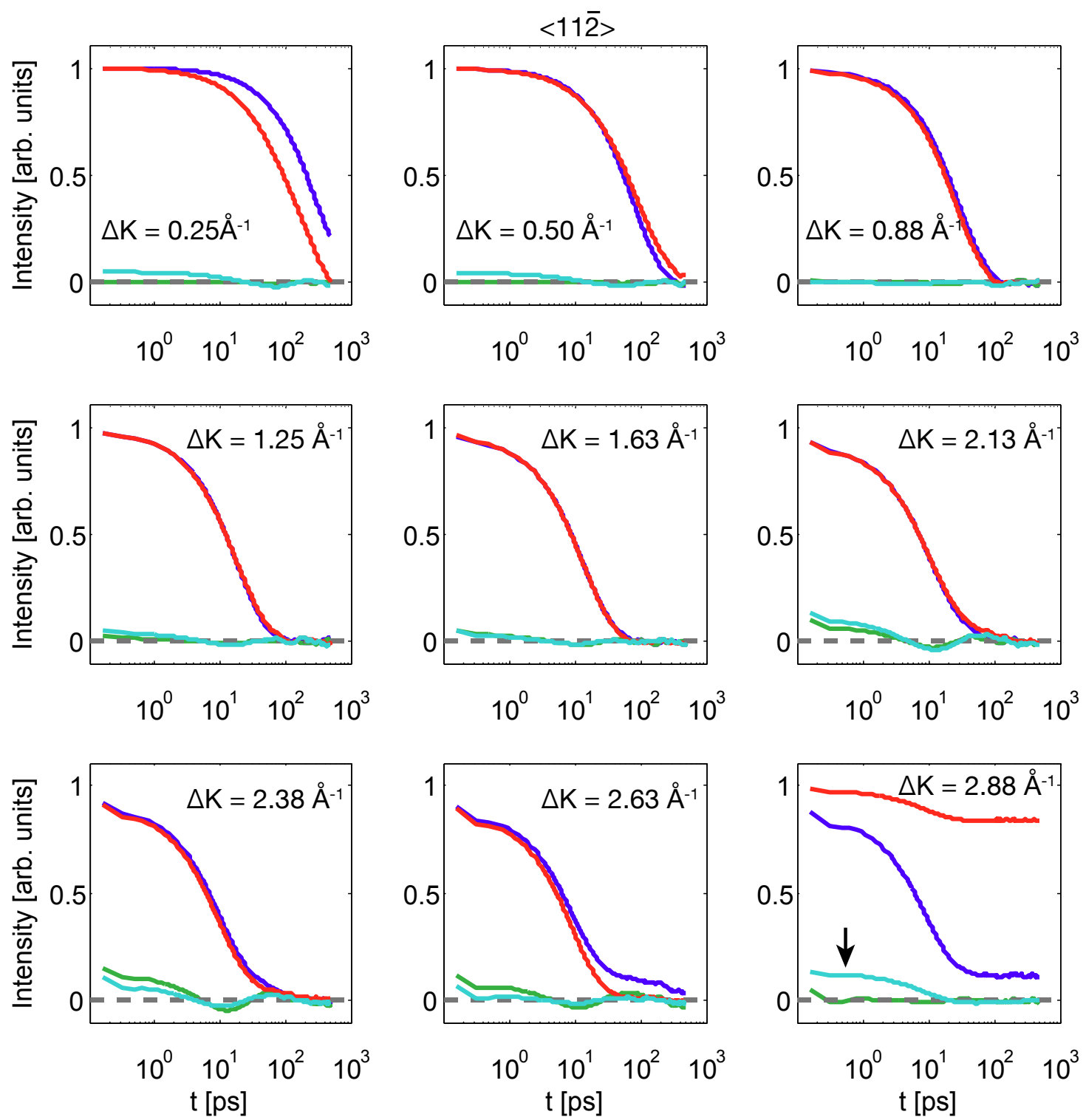

Figure 6.23: Polarisation curves as a function of spin-echo time for MD simulations of 0.033 ML of pyrrole $/ \mathrm{Cu}(111)$ along $<11 \overline{2}>$ without and with pair-wise interactions. The different panels show results at increasing momentum transfers. ISFs from single particle simulations are shown as blue lines, those from simulations including pair-wise interactions as red lines. The residuals after subtracting a single exponential of the functional form $f(t)=a \cdot \exp (-\alpha t)+c$ from either ISFs are shown in green and cyan for the single and multiple particle simulations, respectively.

lineshapes to appear more like a single exponential. The ISFs for simulations including lateral interactions are thus in good agreement with the experimental observation that a single exponential function describes the lineshapes. The only exception is for ISFs near 
the diffraction condition, $\Delta \boldsymbol{K}=2.84 \AA^{-1}$ along $<11 \overline{2}>$, where lateral interactions add a large static component as well as an increased deviation from zero in the residual (arrow), thus explaining the peak in the $\alpha(\Delta \boldsymbol{K})$ curves from simulations shown in Figure 6.18.

In conclusion, lateral interactions change the lineshapes in the ISFs at low and high momentum transfers compared to the lineshapes for non-interacting adsorbates, so that they appear as a single exponential in the experiment. Inter-adsorbate interactions thus reconcile the lineshapes with hopping on multiple sites in the unit cell and confirm jump diffusion between adjacent bridge sites.

\subsection{Lateral changes in the zero point energies of vibrational modes}

In Section 6.5, it was reported that DFT calculations predict a $15 \mathrm{meV}$ barrier between bridge sites and fcc or hcp sites, which is smaller than the apparent activation energy for diffusion observed in Arrhenius experiments.

Sacchi and Jenkins repeated their calculations including the zero point energy (ZPE) to investigate the site dependence of the vibrational modes of pyrrole. Since including the ZPE does not usually influence the outcome of DFT calculations significantly, it is commonly neglected. In the case of pyrrole/ $\mathrm{Cu}(111)$, however, calculations including the ZPE see an increase in the activation barrier on fcc sites by $14 \mathrm{meV}$ and on hcp sites by $28 \mathrm{meV}$, thus doubling and tripling the barrier from simple total energy calculations that do not include the ZPE. The result is shown schematically in Figure 6.24 (A). The rate limiting barrier for pyrrole diffusion is $28+15=43 \mathrm{meV}$ which is in reasonable agreement with the experimentally observed barrier of $53 \pm 4 \mathrm{meV}$.

Figure 6.24 (B) illustrates the contribution of the different vibrational modes of pyrrole to the ZPE difference between adsorption well and rate-limiting barrier. At 87\%, the largest contribution to the ZPE difference is made by the $\mathrm{C}-\mathrm{H}$ and $\mathrm{N}-\mathrm{H}$ out-ofplane bending and ring torsion modes, while $\mathrm{C}-\mathrm{H}$ and $\mathrm{N}-\mathrm{H}$ in-plane bending and ring deformation modes are accountable for the remaining $22 \%$. The $\mathrm{ZPE}$ of $\mathrm{C}-\mathrm{H}$ and $\mathrm{N}-\mathrm{H}$ stretching vibrations do not contribute significantly to the barrier (-9\%), although they make the largest absolute contributions to the adsorption energy. 

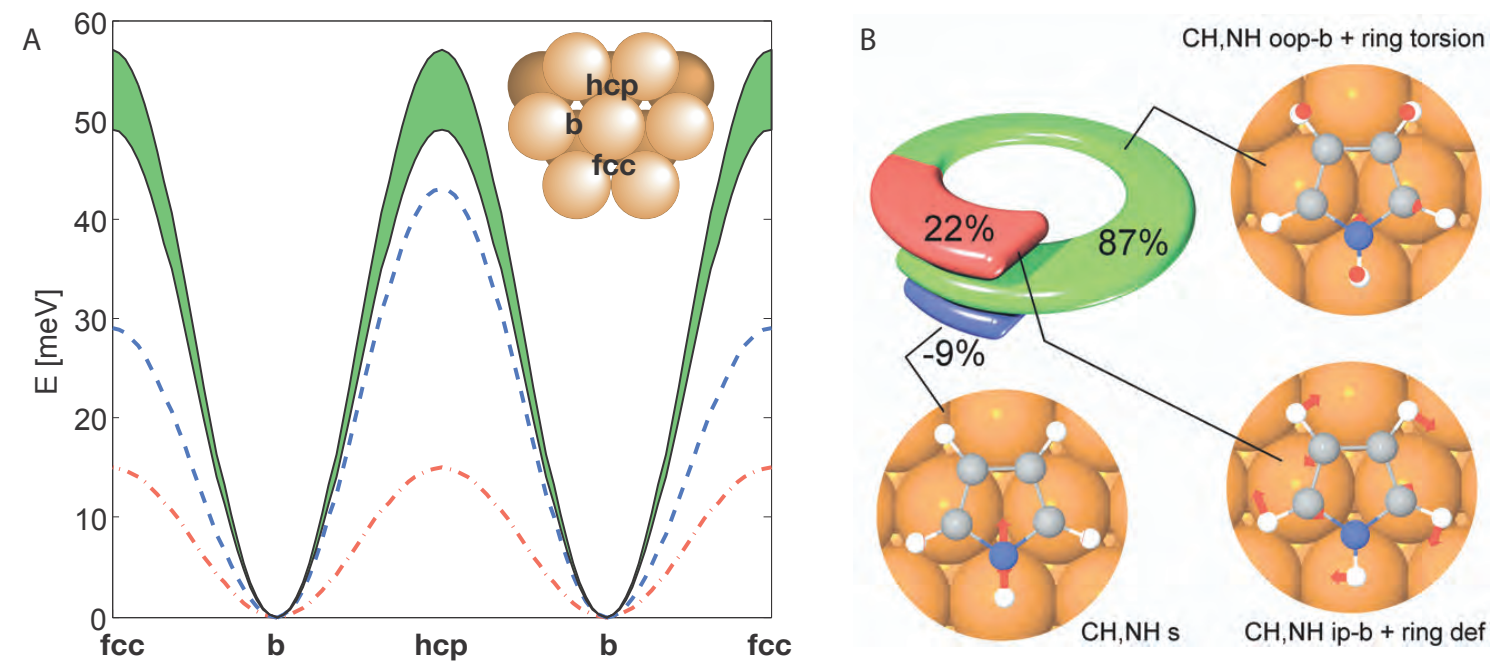

Figure 6.24: The ZPE difference and its influence on the activation barrier. (A) illustrates the corrugation of the PES within the channels around top sites. The inset displays the adsorption sites. Standard DFT calculations (red dash-dotted line) show a small energy barrier. However, when zPEs are included (blue dashed line), good agreement of the rate-limiting barrier with the experimental activation energy from temperature dependent measurements (shaded green area) is obtained. For the purposes of illustration, values for adsorption sites have been connected by sinusoids. (B) shows the contribution of the different vibrational modes of pyrrole to the ZPE difference between adsorption well and transition state (Figure by Sacchi and Jenkins, Cambridge [95]). At 87\%, the largest contribution to the $\mathrm{ZPE}$ difference is made by the $\mathrm{C}-\mathrm{H}$ and $\mathrm{N}-\mathrm{H}$ out-of-plane bending $(\mathrm{CH}, \mathrm{NH}$ oopb) and ring torsion modes, while $\mathrm{C}-\mathrm{H}$ and $\mathrm{N}-\mathrm{H}$ in-plane bending $(\mathrm{CH}, \mathrm{NH}$ ip-b) and ring deformations (ring def) modes are accountable for the remaining $22 \%$. The $\mathrm{ZPE}$ of $\mathrm{C}-\mathrm{H}$ and $\mathrm{N}-\mathrm{H}$ stretching vibrations $(\mathrm{CH}, \mathrm{NH}$ s) do not contribute significantly to the barrier $(-9 \%)$.

\subsection{Conclusions and outlook}

In this chapter, the adsorption behaviour and motion of pyrrole on the hexagonal $\mathrm{Cu}(111)$ surface has been characterised. DFT calculations show that pyrrole adsorbs in a flat-lying geometry at low coverages, in agreement with the adsorption geometry reported for pyrrole on many other transition metal surfaces. TDS experiments further show that pyrrole does not decompose on $\mathrm{Cu}(111)$ and adsorbs/desorbs reversibly at temperatures $\leq 200 \mathrm{~K}$.

Pyrrole is found to move predominantly in single jumps between adjacent bridge sites on $\mathrm{Cu}(111)$ with an apparent activation energy of $53 \pm 4 \mathrm{meV}$. De Gennes narrowing indicates the presence of strong lateral interactions, thus requiring MD simulations to 
describe the experimental results fully, as the lateral interactions influence the lineshapes in the ISFs. MD simulations provide a good description of the experimental $\alpha(\Delta \boldsymbol{K})$ curves and the temperature dependence of the diffusive motion. DFT calculations by Sacchi and Jenkins from the Department of Chemistry at Cambridge confirm bridge sites as the preferred adsorption sites. Their calculations have demonstrated that the apparent activation energy for diffusion is largely due to a site dependence in the zero point energies of the vibrational modes of pyrrole. Interestingly, the largest contribution to the ZPE difference is made by the $\mathrm{C}-\mathrm{H}$ and $\mathrm{N}-\mathrm{H}$ out-of-plane bending and ring torsion modes that are not directly involved in the diffusion. In order to study the effect of quantum modes experimentally, HeSE measurements of deuterated pyrrole/ $\mathrm{Cu}(111)$ could be performed. The difference in mass between pyrrole and deuterated pyrrole would be expected to result in an altered activation barrier for diffusion.

Comparing MD simulations with experimental data, it becomes evident that the interaction potential for lateral repulsion between pyrrole molecules is more complex than a simple dipole-dipole interaction potential. Theoretical studies could be performed to investigate the functional form of the interaction potential further. 


\section{Chapter 7}

\section{The diffusive motion of thiophene on $\mathrm{Cu}(111)$}

Following the investigation of the surface dynamics of two five-membered aromatic adsorbate species - cyclopentadienyl $(\mathrm{Cp})$ and pyrrole - on $\mathrm{Cu}(111)$ in Chapters 5 and 6, this chapter studies the diffusive motion of another, related molecule, thiophene, on the same hexagonal substrate. Thiophene, $\mathrm{C}_{4} \mathrm{H}_{4} \mathrm{~S}$, is a versatile chemical component with many useful applications, for example as a component in chemical dyes or self-assembled superstructures, as part of electronic and optoelectronic devices and in conductivity-based sensors [137].

The interaction of thiophene with metal surfaces has been studied extensively, stimulated both by a desire to understand the fundamental principles underlying adsorbate bonding [138], and by the necessity for investigation of the nature of catalyst poisoning by sulfur-containing substances [139]. Thiophene and other sulfur-containing molecules are common contaminants in fuels and oil-derived products. The combustion of these substances not only results in the release of sulfur oxides, $\mathrm{SO}_{x}$, causing acid rain corroding buildings and adversely affecting plants, but also deactivates catalysts used to remove $\mathrm{CO}$ and NO after combustion [140].

Just like pyrrole, thiophene is a neutral aromatic ring compound in the gas phase. In contrast to the molecules studied in the previous chapters, however, thiophene has two free lone electron pairs on the sulfur atom, of which one is not delocalised in the aromatic ring. Consequently, the molecule can bond to a substrate either in a flat-lying geometry through van der Waals forces between the aromatic ring and the surface, or in a tilted adsorption geometry, forming a chemical bond between the free lone electron pair and 
the substrate. Thiophene thus exhibits more diverse adsorption behaviour than Cp or pyrrole, making it an interesting model system from a fundamental science viewpoint. In addition, thiophene and thiophene-related molecules have great potential as components in the microelectronics industry - forming self-organised thin films on metal substrates thanks to their mobility on the surface - and can be used in electronic devices [139]. It is therefore desirable to understand the interaction of thiophene with metal surfaces and gain insight into the motion of this adsorbate.

This chapter presents a combined helium-3 spin-echo (HeSE) and molecular dynamics study of the diffusive motion of thiophene/ $\mathrm{Cu}(111)$. The adsorption behaviour of this adsorbate system is investigated using desorption and helium atom scattering experiments, facilitating the comparison of HeSE results with the literature. HeSE experiments reveal complex surface dynamics with an interplay of several processes. As for Cp and pyrrole, the experimental study of thiophene diffusion on $\mathrm{Cu}(111)$ is supplemented by firstprinciples density functional theory calculations carried out by Sacchi and Jenkins from the Department of Chemistry at Cambridge.

\subsection{Literature background}

This section gives an overview over the literature on the experimentally determined structure of thiophene and its adsorption behaviour on metal surfaces, with a particular view on $\mathrm{Cu}(111)$.

\subsubsection{The structure of thiophene}

The structure of thiophene was first investigated by Schomaker and Pauling in 1939 using electron diffraction [141]. In a more detailed structural analysis, Bak et al. performed microwave spectroscopy of thiophene and four of its deuterated isotopes, comparing the transitions observed in the spectra of the different species to deduce bond lengths and angles [142]. The resulting dimensions of thiophene are summarised in Figure 7.1. Due to the aromatic nature of the molecule, thiophene is planar in the gas phase. Compared with the geometry of Cp (cf. Figure 5.1) and pyrrole (cf. Figure 6.1), thiophene has a significantly more distorted pentagonal form, with the $\mathrm{S}-\mathrm{C}$ bonds lengthened compared to the corresponding bonds in $\mathrm{Cp}$ and pyrrole. The $\mathrm{C}-\mathrm{S}-\mathrm{C}$ bond angle of $92^{\circ}$ is much smaller than that of a regular pentagon $\left(108^{\circ}\right)$ while the $\mathrm{S}-\mathrm{C}-\mathrm{C}$ and $\mathrm{C}-\mathrm{C}-\mathrm{C}$ angles are 
wider. With the mass of sulfur significantly larger than that of carbon, the centre of mass of the molecule is shifted from the geometric centre towards the sulfur atom.

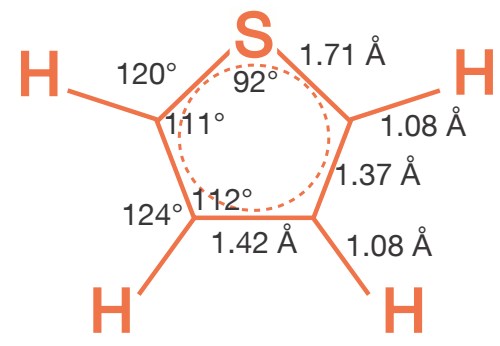

Figure 7.1: Schematic illustration of the structure of thiophene. The bond lengths and angles have been determined by Bak et al. [142]).

\subsubsection{Thiophene adsorption on $\mathrm{Cu}(111)$}

Thiophene adsorption has previously been studied on a vast range of metal surfaces by means of several different techniques. Only a subset of relevant publications is discussed here, focussing predominantly on the adsorption on $\mathrm{Cu}(111)$.

A recurring question in the literature is that of the tilt angle thiophene has on a particular substrate. For example, a near-edge X-ray absorption fine structure (NEXAFs) study on $\operatorname{Pt}(111)$, comparing the $\sigma^{*}$ and $\pi^{*}$ resonances at grazing and normal X-ray incidence, has shown a phase transition from a configuration tilted away from the surface by $\sim 40^{\circ}$ at temperatures below $180 \mathrm{~K}$ to a parallel-bonded geometry [143] at higher temperatures. On $\mathrm{Cu}(100)$, Sexton observed a change from flat-lying to tilted adsorbates with increasing coverage of thiophene [121]. In an electron energy loss spectroscopy (EELS) study, it was found that only out-of-plane modes are active at low coverages, whereas both out-of-plane and in-plane modes were observed at coverages closer to a monolayer (ML). Comparing the results to those for pyrrole and furan, which exhibit a bilayer formation, the author concludes that thiophene forms a compression phase on $\mathrm{Cu}(100)$, moving from flat-lying adsorbates at low coverage to tilted molecules at higher coverages. A flat-lying configuration at low coverages of thiophene $/ \mathrm{Cu}(100)$ was later confirmed in NEXAFs measurements at $92 \mathrm{~K}$ by Imanishi et al. [144], as well as in a comparative low-temperature $(9 \mathrm{~K})$ scanning tunnelling spectroscopy study by Gaudioso and Ho, drawing conclusions about the adsorption geometry of the molecule from its height above the surface [122]. 
A number of research groups have investigated the adsorption of thiophene on $\mathrm{Cu}(111)$ over the past three decades. The first experimental investigation was reported by Richardson and Campuzano who carried out photoemission experiments to study the adsorption geometry of thiophene [145]. The authors reported large exposures, on the order of $10^{-3}$ mbar s, required to attenuate the surface state of $\mathrm{Cu}(111)$ upon thiophene adsorption at room temperature. In the normal emission spectra, they observed three thiophene induced features changing in intensity with the emission angle. In particular, the intensity of the first band is strongest at a $90^{\circ}$ emission angle and decreases for smaller angles, which was suggested to indicate thiophene adsorption in a flat-lying geometry. In addition, a work function decrease of $0.5 \mathrm{eV}$ upon adsorption has been reported.

In 1998, two independent X-ray absorption spectroscopy studies of thiophene $/ \mathrm{Cu}(111)$ were published. Imanishi et al. investigated the thiophene adsorption geometry and electronic properties by means of S K-edge X-ray absorption fine structure measurements, specifically surface extended X-ray absorption fine structure (SEXAFs) and NEXAFs [146]. After dosing 5 Langmuir $\left(1 \mathrm{~L}=1.3 \cdot 10^{-6}\right.$ mbar s) of thiophene at $220 \pm 10 \mathrm{~K}$, corresponding to $0.07 \mathrm{ML}$, the sample was cooled to $92 \pm 2 \mathrm{~K}$ for spectroscopic measurements. In NEXAFS experiments comparing normal X-ray incidence and a grazing incidence angle of $15^{\circ}$, the $\sigma^{*}(\mathrm{~S}-\mathrm{C})$ resonance was found most enhanced at normal incidence, while the $\pi^{*}$ resonance was strongest at grazing incidence. SEXAFs measurements at the same angles of incidence showed strong polarisation dependence of the $\mathrm{S}-\mathrm{C}$ coordination. The authors conclude that thiophene adsorbs in a flat-lying orientation on $\mathrm{Cu}(111)$, accompanied by a charge transfer from the $\mathrm{Cu}(111)$ substrate to the adsorbate $\pi^{*}$ orbital of $0.5 \pm 0.2$ electrons as determined by a $55^{\circ}$ NEXAFs measurement. In curve fitting analysis of the SEXAFs data, a S-Cu distance of $2.50 \pm 0.02 \AA$ was found.

Milligan et al. carried out a similar study, investigating thiophene/Cu(111) using NEXAFS and normal incidence X-ray standing wavefield absorption (NIXSW) [147]. A thiophene overlayer of $0.06 \pm 0.01 \mathrm{ML}$, as determined by X-ray photoelectron spectroscopy (XPS), was prepared by dosing at $100 \mathrm{~K}$ and subsequent annealing to $150 \mathrm{~K}$ to desorb multilayers and part of the monolayer. NEXAFs measurements at $19.5^{\circ}$ (grazing) and normal incidence angles showed predominantly $\pi^{*}$ resonance at grazing incidence and $\sigma^{*}(\mathrm{C}-\mathrm{S})$ resonance at normal incidence, in agreement with Imanishi et al.'s findings. The authors conclude that the molecule adsorbs flat-lying to within $20^{\circ}$. Using NIXSW, triangulating the results of two experiments with different reflecting planes, the sulfur atom of thiophene is found to adsorb on top of a copper atom with a $\mathrm{S}-\mathrm{Cu}$ distance of $2.60 \pm 0.05 \AA$, in close agreement with Imanishi et al.'s results. Thiophene multilayers 
showed no significant angle dependence in NEXAFs measurements, implying randomly oriented molecules.

In 2001, Milligan et al. published a second, more comprehensive study of the adsorption behaviour of thiophene/ $\mathrm{Cu}(111)$, using NIXSW and NEXAFS as well as thermal desorption spectroscopy (TDS) to map out the coverage-dependent adsorption geometry [138]. Low energy electron diffraction (LEED) experiments showed no ordered overlayers of thiophene on $\mathrm{Cu}(111)$. In TDS measurements of the reversibly adsorbing/desorbing thiophene, reproduced in Figure $7.2(\mathrm{~A})$, four distinct peaks were observed upon heating several coverages of thiophene from $110 \mathrm{~K}$ at a heating rate of $0.5 \mathrm{~K} / \mathrm{s}$. A small peak at $297 \mathrm{~K}$, saturating at an exposure of $6.0 \cdot 10^{-7}$ mbar s, was reported to grow with the roughness of the surface and thus attributed to thiophene desorption from defect states. With increasing coverage, a peak at $234 \mathrm{~K}$ became apparent, which the authors termed $\alpha$-state. After saturation of the $\alpha$-state peak at $6 \cdot 10^{-6} \mathrm{mbar}$ s, two further peaks appeared simultaneously, a $\beta$-state peak at $173 \mathrm{~K}$ saturating at $14.4 \cdot 10^{-6} \mathrm{mbar} \mathrm{s}$ exposures, and a multilayer peak at $157 \mathrm{~K}$ that does not saturate. Using Auger intensity measurements the saturation coverages of the two sub-monolayer peaks $\alpha$ and $\beta$ were determined as $0.08 \pm 0.03$ and $0.14 \pm 0.03 \mathrm{ML}$, respectively.

In Milligan et al.'s NIXSW experiments, the sulfur atom was found to adsorb on top sites with a S-Cu distance of $2.62 \pm 0.03 \AA$ in the $\alpha$-state, confirming their previous results. In the $\beta$-state, the adsorption site remained unchanged but the $\mathrm{S}-\mathrm{Cu}$ distance increased to $2.83 \pm 0.05 \AA$, showing a more weakly bound species, in agreement with the TDS data. The authors reported some degree of uncertainty in their measurements which they attributed to either a slight displacement of the sulfur atom from top sites or vibrational motion parallel to the surface. Coverage-dependent NEXAFs measurements were performed using a peak-fitting analysis to determine the adsorption angle from data at normal and $19.5^{\circ}$ (grazing) incidence. In the low coverage state of thiophene adsorbed on defect sites, a flat adsorption geometry with an angle of less than $7^{\circ}$ was found. With increasing coverage, the molecule tilts away from the surface, at an average tilt of $26 \pm 5^{\circ}$ in the $\alpha$-state and $44 \pm 6^{\circ}$ in the $\beta$-state, as illustrated in the figure reproduced in Figure 7.2 (B). Thiophene thus appears to form a compression phase upon saturation of the $\alpha$-state instead of a bilayer configuration, analogous to Sexton's observations for thiophene on $\mathrm{Cu}(100)$ [121].

Comparing earlier work [145-147] with Milligan et al.'s coverage dependent study [138], good agreement is found. The early photoemission experiments by Richardson and 

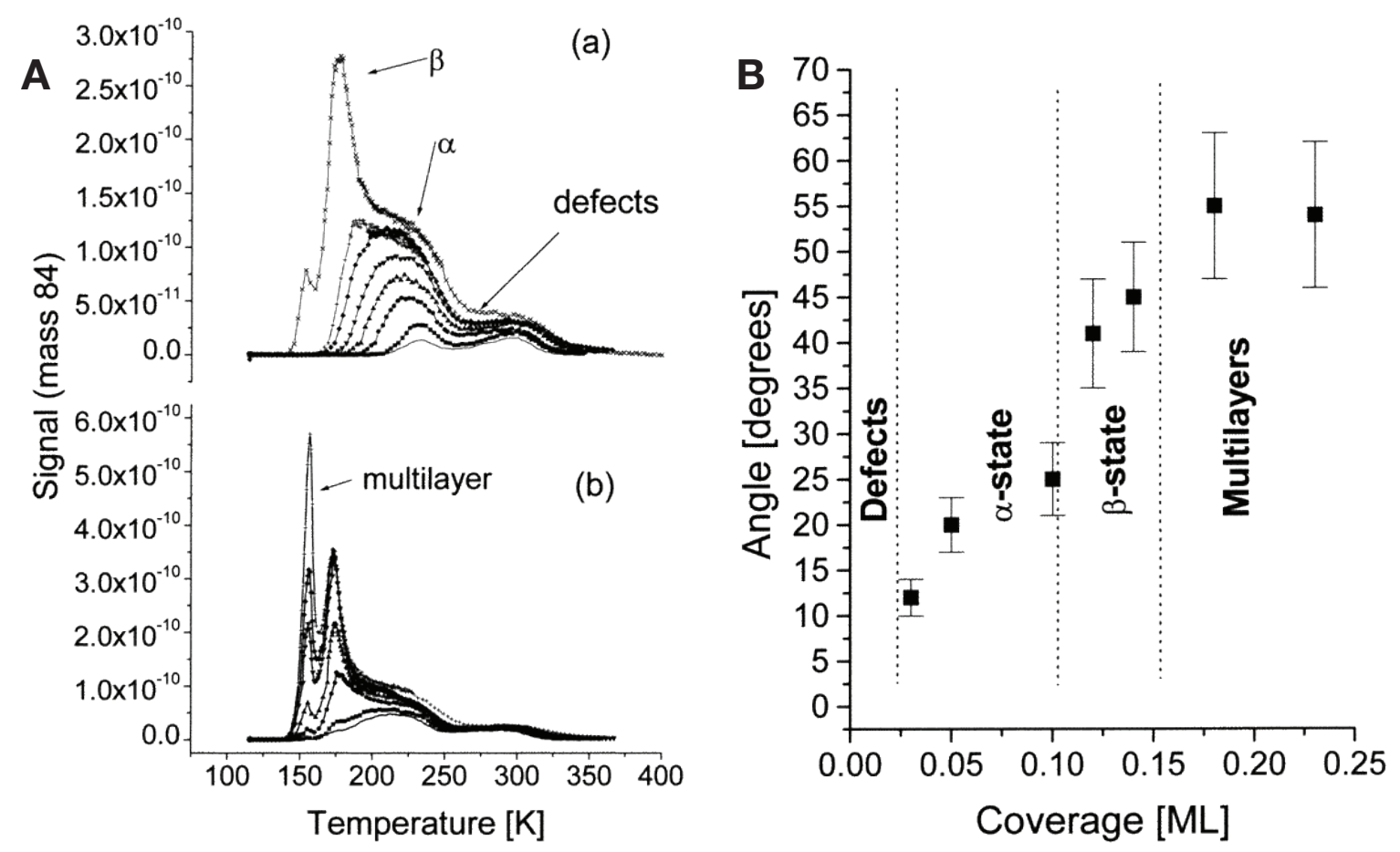

Figure 7.2: TDS and NEXAFS results for thiophene/ $\mathrm{Cu}(111)$, reproduced from [138]. (A) shows TDS measurements monitoring thiophene (mass $84 \mathrm{amu}$ ) desorption during heating at a rate of $0.5 \mathrm{~K} / \mathrm{s}$ for different initial coverages. Two different dosing methods had been applied, back-filling the vacuum chamber for data shown in panel (a), and line-of-sight dosing for data shown in (b). Four desorption peaks can be distinguished, labelled defects, $\alpha, \beta$ and multilayer. (B) summarises the molecule tilt angles as a function of coverage, calculated from NEXAFs data by fitting the measured peaks for a range of different thiophene overlayers.

Campuzano appear to be in the defect-site state reported by Milligan et al., explaining the rather large exposures required for adsorption at $\sim 300 \mathrm{~K}$. A flat-lying adsorption geometry in this low coverage state was reported by both groups. The two studies published in 1998 by Imanishi et al. [146] and Milligan et al. [147] both compare data at two angles of incidence and conclude that thiophene adsorbs in an approximately flatlying geometry (specified as $\leq 20^{\circ}$ by Milligan et al.) in a coverage region corresponding to the $\alpha$-state. While the 2001 publication by Milligan et al. equally only shows data for two incidence angles, more detailed analysis of the peak shape and height allowed for a more accurate determination of the tilt angle in this coverage regime, and an angle of $26 \pm 5^{\circ}$ was reported. While not precisely flat-lying, the qualitative findings presented in the earlier publications are in reasonable agreement with this value. 
In summary, experimental studies show that thiophene adsorbs/desorbs reversibly on $\mathrm{Cu}(111)$ at temperatures below $300 \mathrm{~K}$. No ordered overlayer structures have been observed, but two different adsorption states, $\alpha$ and $\beta$, exhibiting different tilt angles were found. While thiophene adsorbs with its ring approximately parallel to the surface at very low coverages $(\leq 0.02 \mathrm{ML})$, increased tilt angles of $26 \pm 5^{\circ}$ and $44 \pm 6^{\circ}$ were observed in the $\alpha$ - and $\beta$-states, respectively.

\subsection{Experimental methods}

Dynamics and diffraction measurements were performed in the HeSE spectrometer (cf. Chapter 2) using a beam energy between $8.2 \mathrm{meV}$ and $8.3 \mathrm{meV}$, while MiniScat was used for TDS experiments (cf. Chapter 3, "original setup"), at a beam energy of $64 \mathrm{meV}$. The background pressure in the scattering chamber was $5 \cdot 10^{-11}$ mbar in the HeSE spectrometer (Leybold Ionivac ion gauge [48]) and $3 \cdot 10^{-10}$ mbar in MiniScat (Edwards ion gauge [49]).

A single crystal $\mathrm{Cu}(111)$ sample (Surface Preparation Laboratory, Netherlands) was mounted on a six-axis manipulator in each scattering chamber and cleaned by $\mathrm{Ar}^{+}$ sputtering ( $800 \mathrm{eV}, 20$ minutes at $300 \mathrm{~K}$ ) and annealing ( $800 \mathrm{~K}$ for 30 seconds). Surface cleanliness was confirmed using the specular helium reflectivity, exceeding $37 \%$. The crystal azimuth was aligned using the known diffraction pattern of a CO overlayer [27].

Before dosing, thiophene (Fluka, puriss. $\geq 99.5 \%$ ) was purified by repeated freezethaw cycles in high vacuum to remove volatile impurities. In MiniScat, the sample was dosed at approximately $100 \mathrm{~K}$ by backfilling the chamber with an overpressure of $3 \cdot 10^{-9}$ mbar thiophene (as read off the gauge). In the HeSE spectrometer, two different dosing methods were employed. Uptake measurements were performed by backfilling the chamber to allow proper comparison with MiniScat data, while the sample was prepared using a line-of-sight dosing method for dynamics experiments, positioning a dosing tube in front of the crystal surface while the sample was kept at $160 \mathrm{~K}$. In both spectrometers, the purity of the dosed thiophene was checked in a quadrupole mass spectrometer and the dosing process monitored using the specular helium signal. The thiophene overlayers were observed to remain stable for 18 hours at temperatures between $100 \mathrm{~K}$ and $300 \mathrm{~K}$ and could be removed from the surface by flash-heating the sample to $500 \mathrm{~K}$. 


\subsection{Adsorption behaviour}

To study the adsorption behaviour of thiophene $/ \mathrm{Cu}(111)$, the specular helium signal was monitored during dosing. Two typical uptake curves are shown in Figure 7.3, at $100 \mathrm{~K}$ measured on MiniScat in the left panel, and at $160 \mathrm{~K}$ performed in the HeSE spectrometer in the right panel. While care is required when comparing exposure levels between the two machines as pressures are recorded by different gauges, the shape of the curve and the attenuation level of the signal can be compared.
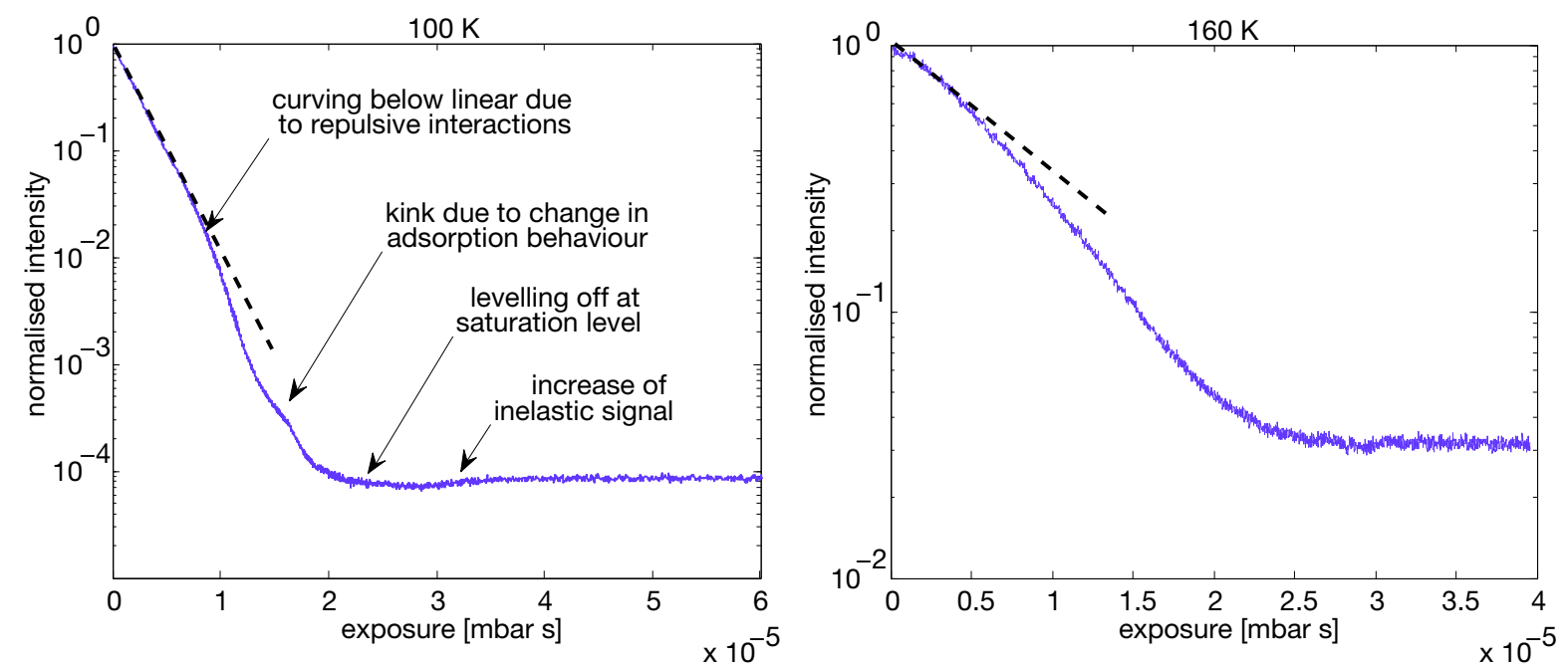

Figure 7.3: Uptake curves for thiophene/Cu(111) at $100 \mathrm{~K}$, recorded on MiniScat (left), and at $160 \mathrm{~K}$, recorded on the spin-echo spectrometer (right). In both cases, the curve falls below a straight line (compare with dashed line), implying repulsive interactions between the adsorbates. At $160 \mathrm{~K}$, saturation is reached at a specular attenuation of approximately $\mathrm{I}_{0} / 30$, while the saturation level at the lower temperature is $\mathrm{I}_{0} / 10000$, indicating that a higher coverage of thiophene is achieved. Note that the exposure levels should not be compared quantitatively as different gauges were used.

At $100 \mathrm{~K}$, thiophene adsorbs until saturation is reached at a specular attenuation of approximately $\mathrm{I}_{0} / 10000$. Similarly to observations for pyrrole/Cu(111) (cf. Chapter 6), the curve falls below a straight line at low exposures suggesting repulsive interactions between the adsorbate particles [15]. A kink at $\sim \mathrm{I}_{0} / 4000$ indicates a change in the adsorption behaviour, possibly due to a transition from the co-existing $\alpha$-and $\beta$-states as defined by Milligan et al. [138] to disordered multilayers, as confirmed by TDS measurements presented below. The small increase in the signal after the saturation level has been reached is an artifact from a rising inelastic signal due to the fairly high 
beam energy used $(64 \mathrm{meV})$. At $160 \mathrm{~K}$ - the temperature at which most dynamics measurements have been recorded - saturation is reached at an attenuation level of $\sim \mathrm{I}_{0} / 30$. Like at $100 \mathrm{~K}$, repulsive lateral interactions show as a downwards sloped curve.

To relate specular attenuation levels along an uptake curve to the different adsorption regimes described by Milligan et al. [138], TDS measurements were performed for submonolayer coverages of thiophene/ $\mathrm{Cu}(111)$. Figure 7.4 shows spectra recorded for a range of coverages $-\mathrm{I}_{0} / 1.1$ (orange), $\mathrm{I}_{0} / 1.6$ (red), $\mathrm{I}_{0} / 3$ (purple), $\mathrm{I}_{0} / 10$ (blue), $\mathrm{I}_{0} / 25$ (cyan) and $\mathrm{I}_{0} / \sim 4000$ (green) - as well as the specular helium signal for desorption of the $\mathrm{I}_{0} / 10$ dose of thiophene. The different attenuation levels are illustrated on the uptake curve reproduced from Figure 7.3.

The specular helium signal shows a peak at $270 \mathrm{~K}$ corresponding to the signal level of clean $\mathrm{Cu}(111)$, thus indicating that all thiophene is desorbed from the surface at that temperature. The subsequent decrease of the signal is thought to be due to Debye Waller attenuation as thermal motion increases, or alternatively due to slight misalignments from large temperature changes. From the helium-TDS curve it is apparent that all mass $84 \mathrm{amu}$ peaks beyond $270 \mathrm{~K}$ arise from desorption from the manipulator or sample mount rather than the sample itself and thus need to be ignored in the interpretation of the TDS data. The remaining peaks are in good qualitative agreement with the results by Milligan et al. (cf. Figure $7.2(\mathrm{~A})$ ). The highest temperature desorption peak occurs between $240 \mathrm{~K}$ and $260 \mathrm{~K}$ and can be assigned to the $\alpha$-peak found at $234 \mathrm{~K}$ by Milligan et al. In the highest coverage spectrum, a further peak appears at $190 \mathrm{~K}$, corresponding to Milligan et al.'s $\beta$-peak at $173 \mathrm{~K}$, and a foot at $\sim 150 \mathrm{~K}$ might be the onset of multilayer formation, as observed at $157 \mathrm{~K}$ by Milligan et al. [138]. The kink at $\sim \mathrm{I}_{0} / 4000$ in the uptake curve at $100 \mathrm{~K}$ is therefore interpreted as the beginning of multilayer formation, which - in comparison with exposure levels given by Milligan et al. - puts the saturation level of the $\alpha$-state in the region of the $I_{0} / 25$ point in the uptake curve.

From the peaks in thermal desorption spectra the adsorption energies can be estimated using the Redhead equation (cf. Equation 6.1) [131], yielding $E=0.63 \mathrm{eV}$ to $0.69 \mathrm{eV}$ $(61 \mathrm{~kJ} / \mathrm{mol}$ to $67 \mathrm{~kJ} / \mathrm{mol})$ for the $\alpha$-peak and $E \approx 0.50 \mathrm{eV}(48 \mathrm{~kJ} / \mathrm{mol})$ for the $\beta$ peak. While the desorption energy for $\alpha$-bonded thiophene molecules is very similar to that determined for pyrrole on the same substrate, $0.68 \mathrm{eV}$ (cf. Chapter 6), the type of bonding in these two adsorbate systems is different. Pyrrole is physisorbed, bonding predominantly through van der Waals forces and weak $\pi$-bonding in a flat-lying geometry while thiophene adsorbs tilted at an angle to the substrate with a covalent bond 

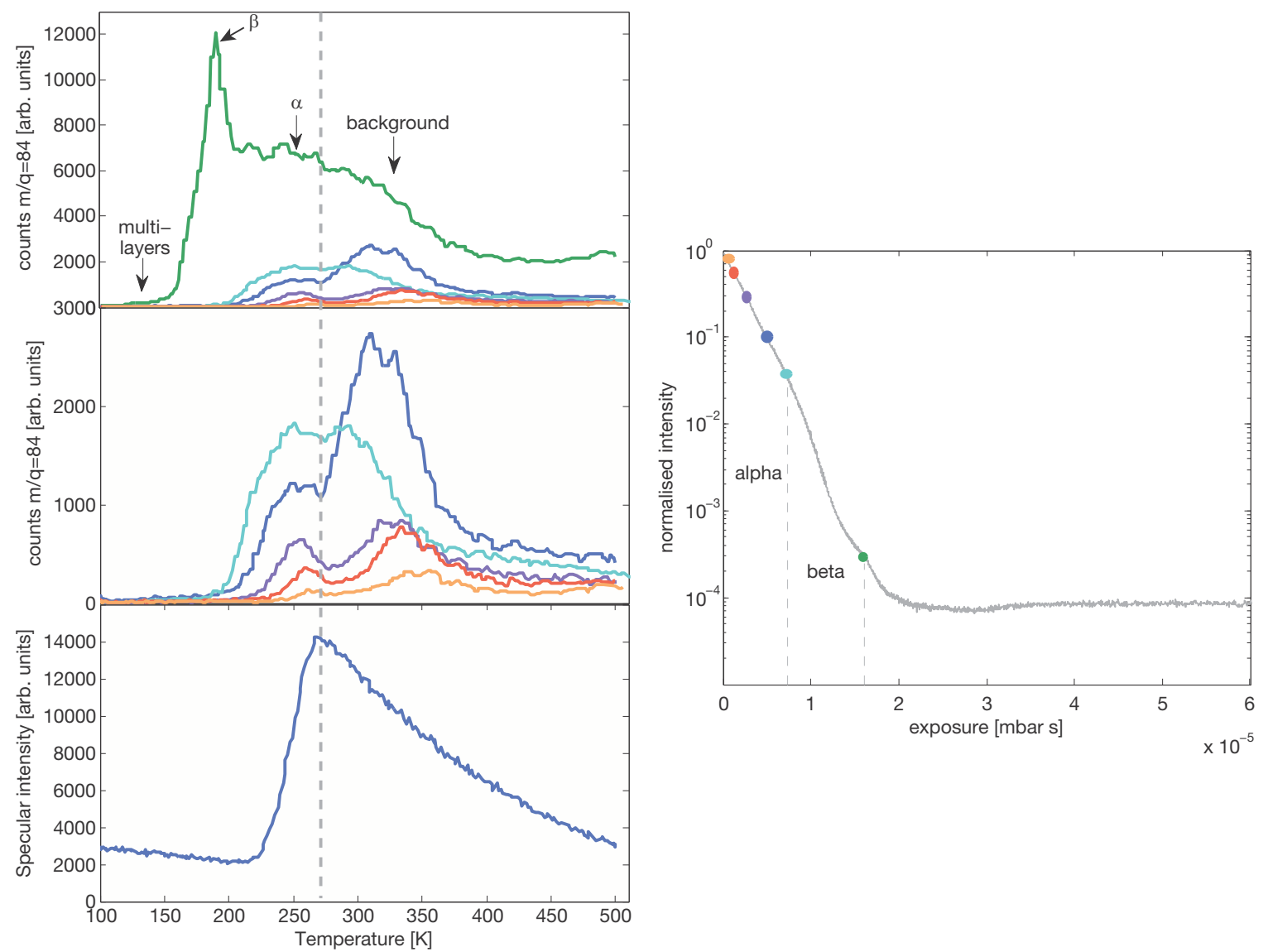

Figure 7.4: Thermal desorption spectra performed on MiniScat for thiophene $/ \mathrm{Cu}(111)$ across a range of coverages, corresponding to $\mathrm{I}_{0} / 1.1$ (orange), $\mathrm{I}_{0} / 1.6$ (red), $\mathrm{I}_{0} / 3$ (purple), $\mathrm{I}_{0} / 10$ (blue), $\mathrm{I}_{0} / 25$ (cyan), $\mathrm{I}_{0} / \sim 4000$ (green). The right-hand side panel illustrates specular attenuation levels on an uptake curve at $100 \mathrm{~K}$ in corresponding colours. The top left panel presents all curves and is labelled to compare the peaks to those reported by Milligan et al. (cf. Figure 7.2 B) [138]. A magnified version of the lower coverage curves is shown in the centre panel while the bottom panel depicts the intensity of the specular helium beam during desorption of the $\mathrm{I}_{0} / 10$ coverage. It becomes apparent from the specular signal intensity that all thiophene is desorbed from the sample at approximately $270 \mathrm{~K}$ (peak), implying that the mass $84 \mathrm{amu}$ signal beyond that temperature is due to desorption from the manipulator and sample mount and therefore must be disregarded in the interpretation of TDS data.

between the sulfur atom and the copper substrate as implied in the adsorption geometry determined by Milligan et al. [138]. The similar interaction strength of a physisorbed and a chemisorbed system can be understood in terms of number of atoms participating in the bonding. In the case of pyrrole, five atoms $(\mathrm{C}$ and $\mathrm{N})$ bond to the substrate, while the contribution of the carbon atoms to the thiophene-substrate bond is small 
(which becomes apparent in the large bond distance in the tilted geometry) and the $\mathrm{S}-\mathrm{Cu}$ interaction dominates.

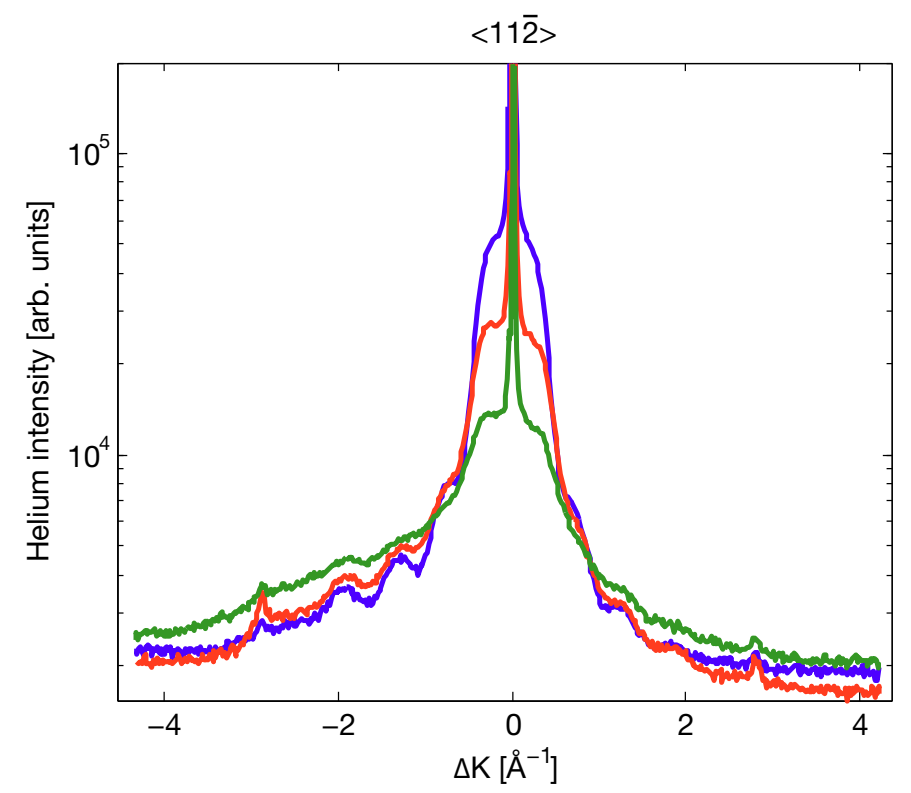

Figure 7.5: Diffraction scans for three coverages of thiophene $/ \mathrm{Cu}(111)$ along $<11 \overline{2}>$, corresponding to specular attenuation levels of $\mathrm{I}_{0} / 3$ at $160 \mathrm{~K}$ (blue), $\mathrm{I}_{0} / 9$ at $160 \mathrm{~K}$ (red) and $\mathrm{I}_{0} / 25$ at $140 \mathrm{~K}$ (green).

In addition to uptake curves and TDS measurements, diffraction scans were recorded for coverages corresponding to those used in the HeSE measurements, given as specular attenuation levels. Three characteristic curves along $<11 \overline{2}>$ are shown in Figure 7.5 for $\mathrm{I}_{0} / 3$ at $160 \mathrm{~K}, \mathrm{I}_{0} / 9$ at $160 \mathrm{~K}$ and $\mathrm{I}_{0} / 25$ at $140 \mathrm{~K}$. All curves show similar features, yet the intensity at $\mathrm{I}_{0} / 25$ at $140 \mathrm{~K}$ is lower. A small diffraction peak at $2.84 \AA^{-1}$ corresponds to the dimensions of the $\mathrm{Cu}(111)$ lattice, most likely caused by defects or adsorbates forming a lattice gas (cf. Section 5.3). In addition, several features can be observed at $0.2 \AA^{-1}, 0.7 \AA^{-1}, 1.2 \AA^{-1}$ and $1.9 \AA^{-1}$. The experiments were repeated $7^{\circ}$ off the azimuth (not shown above), giving identical spectra apart from the diffraction peak at $2.84 \AA^{-1}$ which disappears off $<11 \overline{2}>$. The remaining features are therefore diffraction rings. Since all rings exhibit coverage-independent momentum transfer positions, they must be due to the structure of the individual adsorbates rather than overlayer structures. As illustrated in Equation 2.2, the total scattered amplitude in a diffraction experiment is the product of a form factor, representing individual scattering centres, and a structure factor, which is due to the arrangement of all scattering centres on the substrate and thus coverage-dependent. The diffraction rings in Figure 7.5 can therefore be identified as form factor rings. 


\subsection{Measuring the surface dynamics}

HeSE measurements of the surface motion of thiophene/ $\mathrm{Cu}(111)$ were performed for a range of temperatures between $105 \mathrm{~K}$ and $165 \mathrm{~K}$, along the two principal crystal directions and at three different coverages, corresponding to specular attenuation levels of $\mathrm{I}_{0} / 3, \mathrm{I}_{0} / 9$ and $\mathrm{I}_{0} / 30$.

\subsubsection{Complex lineshapes}

The lineshapes in the intermediate scattering functions (ISFs) are of a complex nature, comprising signatures from several processes occuring on similar timescales. Typical ISFs are shown in Figures 7.6 and 7.7 for polarisation measurements along the $<1 \overline{1} 0>$ and $<11 \overline{2}>$ azimuths, respectively. Experimental data are presented on a logarithmic time axis to illustrate processes occuring on different timescales. Comparing the lineshapes with those observed for $\mathrm{Cp}$ and pyrrole on $\mathrm{Cu}(111)$ (cf. Sections 5.4.1 and 6.4.1), certain familiar features can be distinguished. At short spin-echo times, $t$, and small $\Delta \boldsymbol{K}$ oscillations are observed, possibly due to substrate phonons or adsorbate vibrations as suggested by Milligan et al. [138]. In addition, the data exhibit a very weak fast decay at approximately $t<0.5 \mathrm{ps}$, which could be attributed to intracell diffusion similar to that observed in Section 5.9. However, the signature in the lineshapes is too weak to provide further information about thiophene motion at short times.

The most striking feature in each ISF shown in Figures 7.6 and 7.7 is a slow decay of polarisation with time, indicating the presence of aperiodic motion $[6]^{1}$. To analyse this slow decay it is compared with a single exponential function starting at 0.5 ps to account for the fast decay described above. The residual after subtracting this single exponential from the experimental data shows a systematic variation, falling below zero before rising above, with increasing $\Delta \boldsymbol{K}$, illustrating that a single exponential function is not sufficient to describe the data $>0.5 \mathrm{ps}$. This observation suggests that the ISFs contain signatures of more than one process, either jump diffusion between several sites forming a non-Bravais lattice (cf. Section 2.4.1) [38] or two entirely different types of motion [6]. These two cases can be distinguished by investigating the temperature dependence of thiophene diffusion [132]. Hopping on a non-Bravais lattice would result in a single activation barrier for all decays (cf. Figure 5.8) while one would expect two

\footnotetext{
${ }^{1}$ At low $\Delta \boldsymbol{K}$ values the ISFs are not fully resolved with the beam energy used but the resolution is
} sufficient to determine the curvature of the ISFs. 

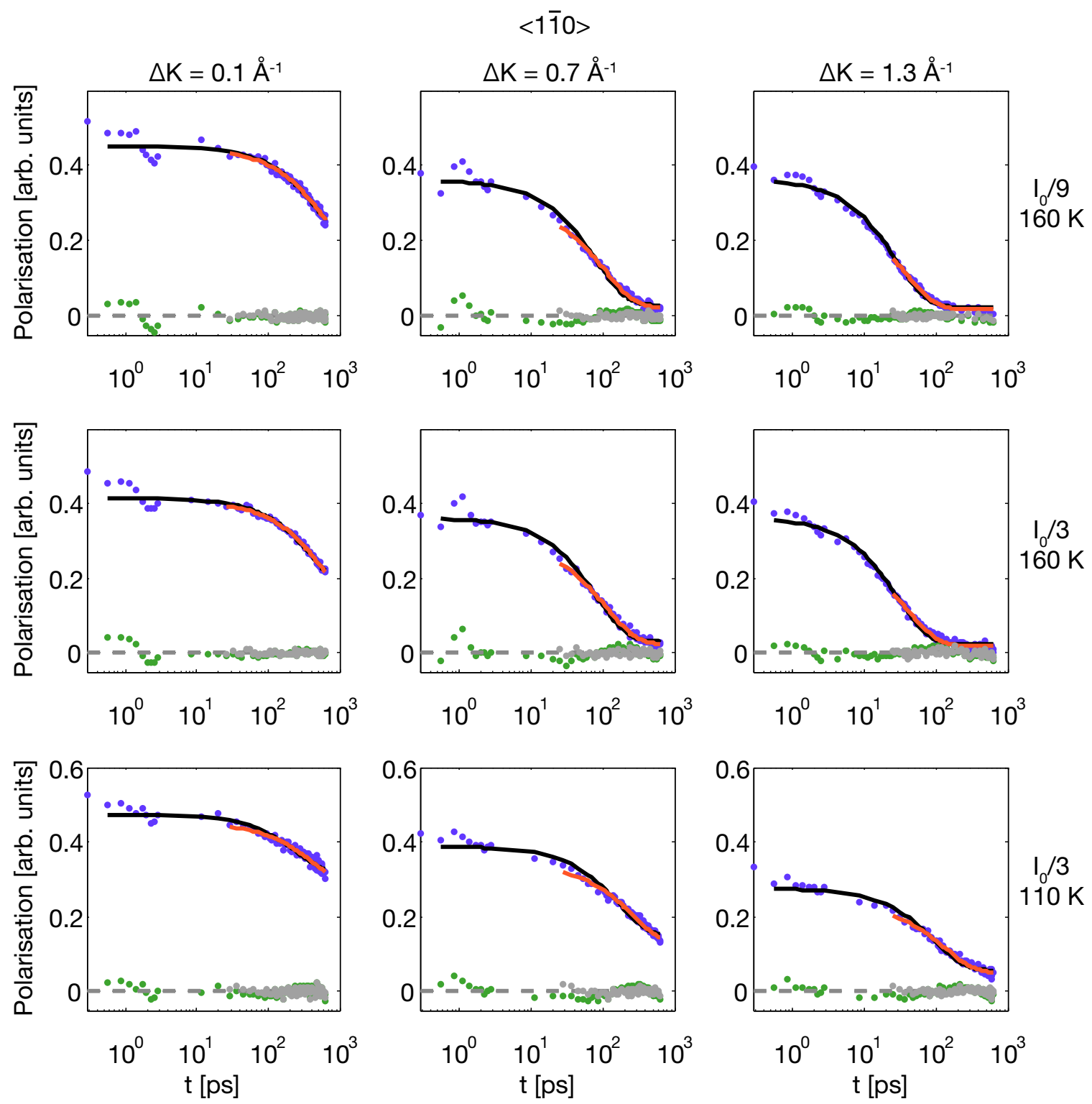

Figure 7.6: Polarisation measurements as a function of spin-echo time, $t$, for thiophene $/ \mathrm{Cu}(111)$ along the $<1 \overline{1} 0>$ direction. Three rows show data for different coverages and different temperatures, as labelled on the right, while the columns represent different momentum transfer values of $0.1 \AA^{-1}, 0.7 \AA^{-1}$ and $1.3 \AA^{-1}$. Experimental data are plotted as blue points, a single exponential through the data from $0.5 \mathrm{ps}$ is shown as a black line and that from $25 \mathrm{ps}$ as a red line. The residual after subtracting either exponential function from the data is shown as green and grey points, respectively.

different modes of motion to exhibit a different temperature dependence for each process. The temperature dependence is investigated in the following section ${ }^{2}$.

\footnotetext{
${ }^{2}$ Note that the red lines and grey dots in Figures 7.6 and 7.7 will be discussed in Section 7.4.3.
} 


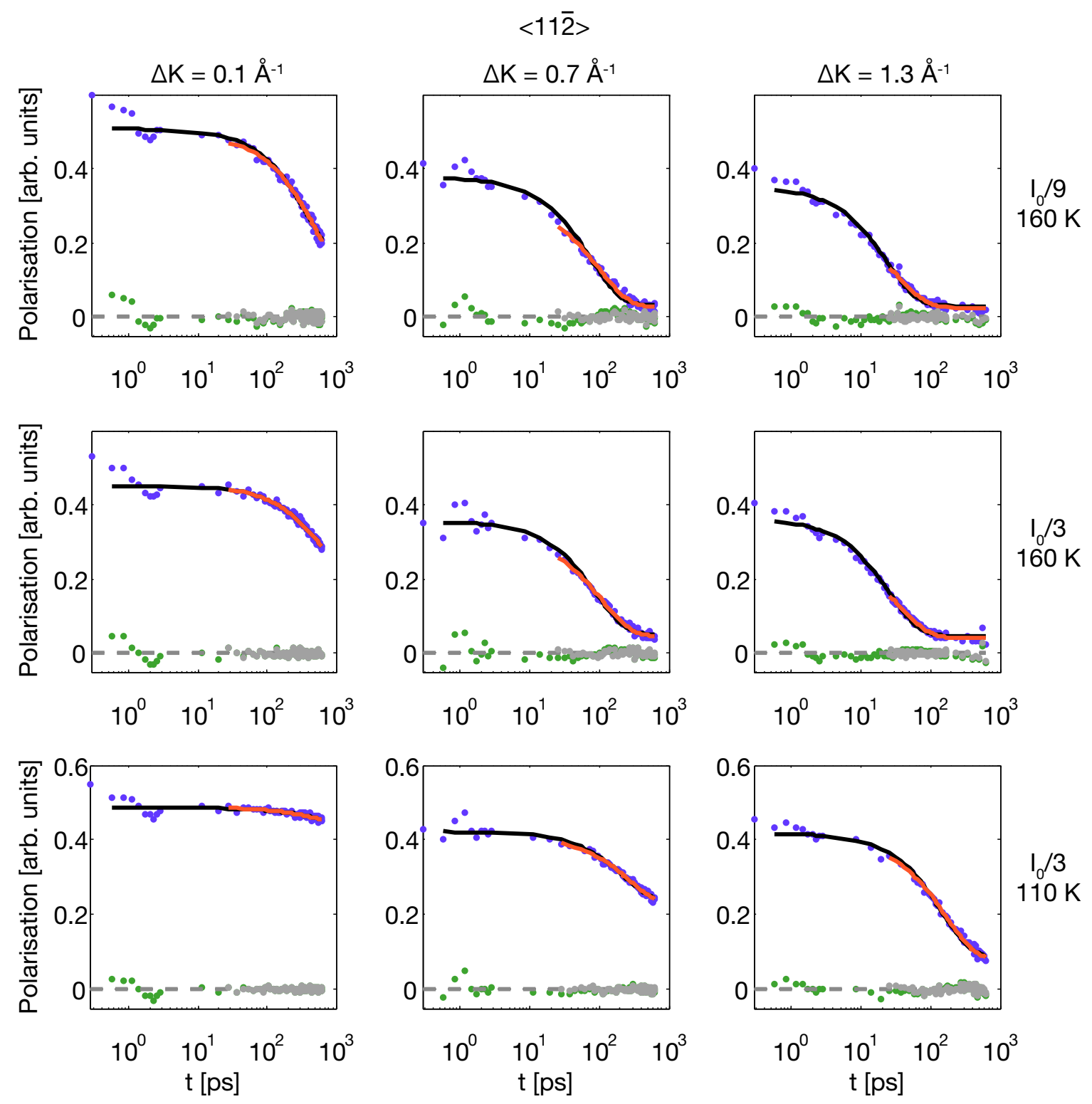

Figure 7.7: Polarisation measurements as a function of spin-echo time, $t$, for thiophene $/ \mathrm{Cu}(111)$ along the $<11 \overline{2}>$ direction. Three rows show data for different coverages and different temperatures, as labelled on the right, while columns represent different momentum transfer values of $0.1 \AA^{-1}, 0.7 \AA^{-1}$ and $1.3 \AA^{-1}$. Experimental data are plotted as blue points, a single exponential through the data from $0.5 \mathrm{ps}$ is shown as a black line and that from $25 \mathrm{ps}$ as a red line. The residual after subtracting either exponential function from the data is shown as green and grey points, respectively.

\subsubsection{Two competing activated processes}

To determine whether the deviation from a single exponential function observed in the ISFs is due to hopping on a non-Bravais lattice or instead indicative of several types of 
adsorbate motion, HeSE measurements were performed at temperatures between $105 \mathrm{~K}$ and $165 \mathrm{~K}$ at a fixed momentum transfer of $0.7 \AA^{-1}$ along $<11 \overline{2}>$. By analysing the data with a single exponential starting at 0.5 ps [132], ignoring the small deviations observed in this momentum transfer region in Figure 7.7, two competing activated processes are observed. Figure 7.8 shows data at two different coverages, corresponding to an attenuation of the helium signal of $\mathrm{I}_{0} / 3$ and $\mathrm{I}_{0} / 9$. An abrupt change in the activation energy is observed at approximately $145 \mathrm{~K}$ in both curves as the single exponential describing the data is dominated by one process at low temperatures and by another, more strongly activated process at higher temperatures, reflected in two discernible straight lines when plotted. The Arrhenius activation energy for the dominant process at low temperatures is approximately $21 \pm 1 \mathrm{meV}$ for $\mathrm{I}_{0} / 3$ and $22 \pm 1 \mathrm{meV}$ for $\mathrm{I}_{0} / 9$, and that for the second type of motion is $56 \pm 3 \mathrm{meV}$ and $62 \pm 4 \mathrm{meV}$, respectively. Combining the data at both coverages yields an average barrier for the process dominating at low temperatures of $22 \pm 2 \mathrm{meV}$ and an effective barrier for the high temperature process of $59 \pm 4 \mathrm{meV}$. The deviation from a single exponential in the experimental lineshapes is thus attributable to the presence of two competing activated processes.

In another recent HeSE study, Paterson et al. have observed a similar change in activation energy for a related adsorbate system, namely ethanethiolate on $\mathrm{Cu}(111)$ [132]. They found the process dominating at high temperatures to be jump diffusion on a Bravais lattice of top sites, while the more weakly activated process was attributed to a form of confined motion: rotation of the alkane tail group around a sulfur-metal anchor point. The signature of rotational motion is a sum of several exponentials of constant dephasing rate varying in intensity with momentum transfer $[132,148]$. Since the actual motion of thiophene can be considered a convolution of rotational motion with translation of the centre of mass, the combined ISF is obtained by taking the product of the contributions from translation and rotation:

$$
I(\Delta \boldsymbol{K}, t)_{\text {total }}=I(\Delta \boldsymbol{K}, t)_{\text {trans }} I(\Delta \boldsymbol{K}, t)_{\text {rot }} .
$$

At sufficiently low temperatures, the contribution from jump diffusion is negligible and the data shows the signature of rotation alone. At large temperatures, beyond the kink in the Arrhenius plot, rotation and jump diffusion were both present, resulting in complex lineshapes. Paterson et al. describe the combined ISF with a single exponential, comparing experimental data with a combined model for jump diffusion and rotation. Overall, they observed only small deviations from a single exponential, indicating that a 


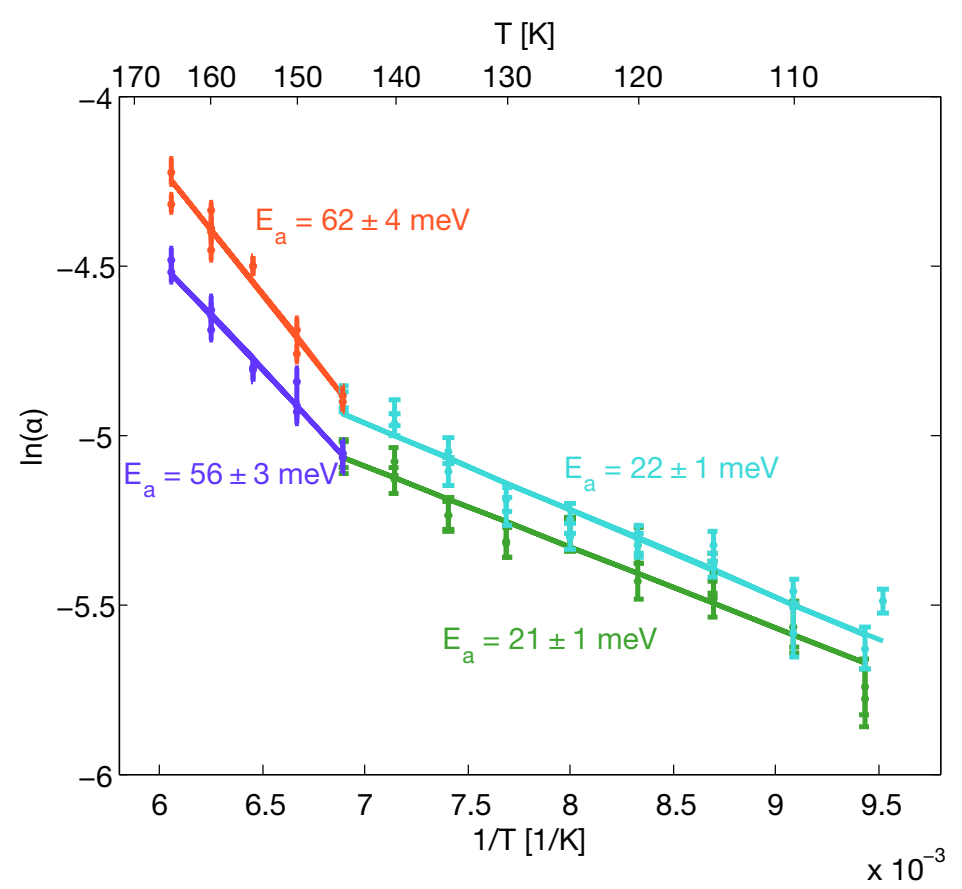

Figure 7.8: Temperature dependent HeSE measurements at $0.7 \AA^{-1}$ along $<11 \overline{2}>$, for two different coverages of thiophene $/ \mathrm{Cu}(111)$. Describing the ISFs by a single exponential function reveals two competing activated processes. The apparent activation barrier for the process dominating at low temperatures is $21 \pm 1 \mathrm{meV}$ at a coverage corresponding to $\mathrm{I}_{0} / 3$ (green) and $22 \pm 1 \mathrm{meV}$ for $\mathrm{I}_{0} / 9$ (cyan). At identical coverages, the more strongly activated process shows a barrier of $56 \pm 3 \mathrm{meV}$ and $62 \pm 4 \mathrm{meV}$, respectively.

sum of several exponentials is reasonably well approximated by a simple single exponential function.

Due to the similarity between thiophene and ethanethiolate, it seems probable that thiophene molecules move in a similar fashion, diffusing between sites and rotating around the sulfur-substrate anchor point. The dynamics measurements in Figures 7.6 and 7.7 were performed at coverages corresponding to $\mathrm{I}_{0} / 3$ and $\mathrm{I}_{0} / 9$, both in the $\alpha$-peak in the TDS measurements reported by Milligan et al., with the implication that the molecules adsorb tilted at an angle of $26 \pm 5^{\circ}$ with respect to the surface plane [138]. While hopping motion involves the breaking of the covalent $\mathrm{S}-\mathrm{Cu}$ bond, rotation should occur more freely as the bond between the carbon atoms and the substrate is longer and therefore weaker. It is reasonable to conclude that the diffusive motion of thiophene is the more strongly activated process. 
As mentioned above, the analytical model for rotational motion predicts a sum of several exponentials [148] and diffusive motion adds one or more further exponentials on similar timescales [6]. Fitting more than two exponentials to experimental data is rather difficult and prone to errors [27]. Although less accurate, a description of each process with a single exponential is therefore the more reliable approach [132]. Across all ISFs, it was found that a sum of two exponentials gives a reasonable description of the lineshapes for thiophene $/ \mathrm{Cu}(111)$ from approximately 0.5 ps. Of the two exponentials, the slow decay shows an approximately sinusoidal $\alpha(\Delta \boldsymbol{K})$ dependence, implying that the slower process corresponds to jump diffusion [38]. The faster component, on the other hand, shows less variation with momentum transfer, yet is strongly influenced by noise in the data and difficult to interpret. For more detailed investigation of rotation, an approach similar to that employed by Paterson et al. is preferable, where the combined lineshape of rotation and jump diffusion is analysed by a single exponential function. In the present thesis, however, the main interest lies in the investigation of the diffusive part of adsorbate motion as studied below.

\subsubsection{The diffusive motion}

To separate the diffusive motion from rotation, the lineshapes in the ISFs were investigated only at long spin-echo times. As the cut-off point is moved to a larger time, $t$, the disagreement between the single exponential and the measured data decreases until approximately $t=25 \mathrm{ps}$, as illustrated in Figures 7.6 and 7.7. The residuals after subtracting two different exponentials, starting at $t=25 \mathrm{ps}$ and $t=0.5 \mathrm{ps}$, from the experimental data are shown in the Figures to illustrate the quality of the fit. Considering, for example, the centre column panels in Figure 7.6, the residuals demonstrate that the exponential from $25 \mathrm{ps}$ is a much improved fit to the data compared with an exponential from $0.5 \mathrm{ps}$, evident in the smaller deviation from zero in the residual for the exponential starting at longer $t$. Note that the activation barrier for diffusive motion, i.e. the more strongly activated part in the Arrhenius plot in Figure 7.8, does not change significantly with the spin-echo time cut-off point.

Figure 7.9 shows the dephasing rate as a function of momentum transfer at $160 \mathrm{~K}$ obtained from single exponentials starting at $t=25 \mathrm{ps}$, compared to exponentials from $t=0.5 \mathrm{ps}$ to illustrate the effect of including the fast process. At very small $\Delta \boldsymbol{K}$ only a small difference between the curves for exponentials from $t=0.5 \mathrm{ps}$ and $t=25 \mathrm{ps}$ is observed, but at larger $\Delta \boldsymbol{K}$ the signature of the fast process becomes dominant, 
resulting in higher values of $\alpha$ and a curve that does not tend towards zero decay at the diffraction position $\left(2.84 \AA^{-1}\right.$ along $\left.<11 \overline{2}>\right)$ when the signatures at short spin-echo times are included. To analyse the diffusive motion of thiophene only the slow component is considered below.

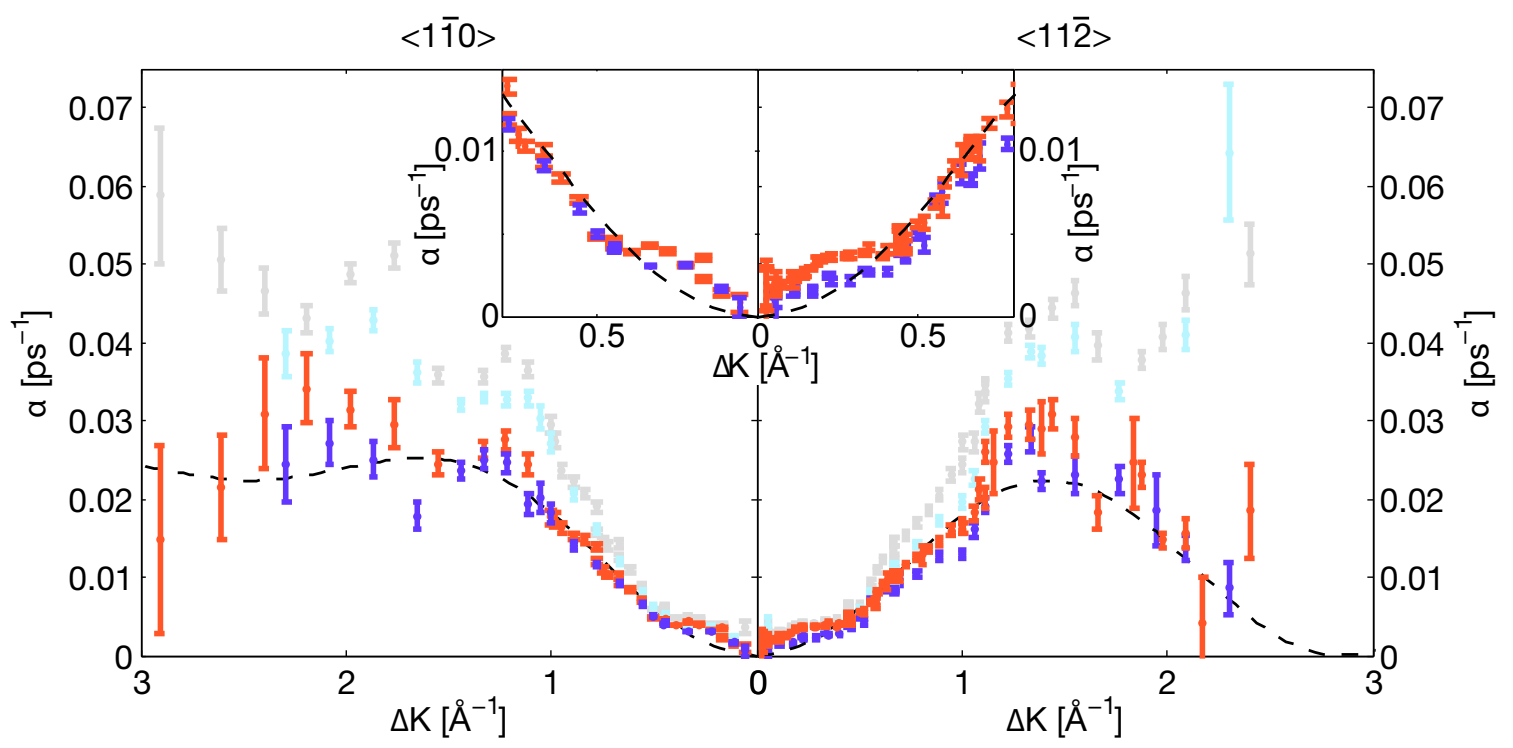

Figure 7.9: $\alpha(\Delta \boldsymbol{K})$ dependence of the diffusive motion of thiophene $/ \mathrm{Cu}(111)$ at $160 \mathrm{~K}$ along two azimuths, $<1 \overline{1} 0>$ and $<11 \overline{2}>$. The dephasing rates for a single exponential from $t=25$ ps are shown for two coverages, corresponding to $\mathrm{I}_{0} / 3(0.015 \mathrm{ML})$ and $\mathrm{I}_{0} / 9$ (0.022 ML) shown in dark blue and red, respectively. Dashed black lines represent the analytical model for jump diffusion between top sites [35], scaled to match the measurements. To illustrate the effect of rotation, an exponential starting at $t=0.5 \mathrm{ps}$ is shown in light blue $\left(\mathrm{I}_{0} / 3\right)$ and grey $\left(\mathrm{I}_{0} / 9\right)$. The inset is a magnified view of the small $\Delta \boldsymbol{K}$ region exhibiting the de Gennes feature.

The curves for diffusive motion exhibit a sinusoidal $\alpha(\Delta \boldsymbol{K})$ dependence, in good agreement with the analytical model for single hops between adjacent top sites [35], shown to match the data in Figure 7.9. HeSE experiments of thiophene/ $\mathrm{Cu}(111)$ thus confirm Milligan et al.'s findings that top sites are the preferred adsorption sites. Small deviations from the simple jump diffusion curves can be found at low $\Delta \boldsymbol{K}$ (cf. inset in Figure 7.9). This peak and dip feature can be identified as de Gennes narrowing [41] - indicating repulsive adsorbate interactions - and is similar to the feature observed for pyrrole (cf. Figure 6.9), yet weaker in intensity. From Equation 2.24, the thiophene coverage can be estimated from the dip position in the experimental curves. At the lower coverage, the dip is located at approximately $0.35 \AA^{-1}$, corresponding to a coverage of $\sim 0.015 \mathrm{ML}$, while the higher coverage curve exhibits a dip at approximately $0.42 \AA^{-1}$, indicating a coverage of $\sim 0.022 \mathrm{ML}$. Using Equation 2.4 and the specular attenuation levels $\mathrm{I}_{0} / 3$ and 
$\mathrm{I}_{0} / 9$, these coverages suggest a scattering cross section of about $490 \pm 70 \AA^{2}$, which is exceptionally large [15]. For comparison, the scattering cross section of Cp has been estimated as $200 \AA^{2}$ (cf. Section 5.3) and that of pyrrole as $350 \AA^{2}$ (cf. Section 6.4.3). A possible explanation for the large cross section of thiophene in a helium beam is an averaged cross section of a molecule rotating relatively freely around the sulfur-copper bond.

Repeating the experiments of $0.015 \mathrm{ML}\left(\mathrm{I}_{0} / 3\right)$ thiophene $/ \mathrm{Cu}(111)$ at $110 \mathrm{~K}$ gives a significantly different result. While $160 \mathrm{~K}$ is at the high temperature end of the Arrhenius curve, $110 \mathrm{~K}$ is in the low temperature region where the fast process dominates over jump diffusion. Figure 7.10 shows the $110 \mathrm{~K}$ data analysed in the same way as the corresponding higher temperature data, with a single exponential starting at $t=25 \mathrm{ps}$ (dark blue) and $t=0.5 \mathrm{ps}$ (light blue). In contrast to observations at $160 \mathrm{~K}$ (cf. Figure 7.9), the shape of the curve in Figure 7.10 does not vary much as the cut-off point for the single exponential is changed. Rotational motion is therefore dominant at this temperature and information about the jump diffusion cannot easily be extracted.

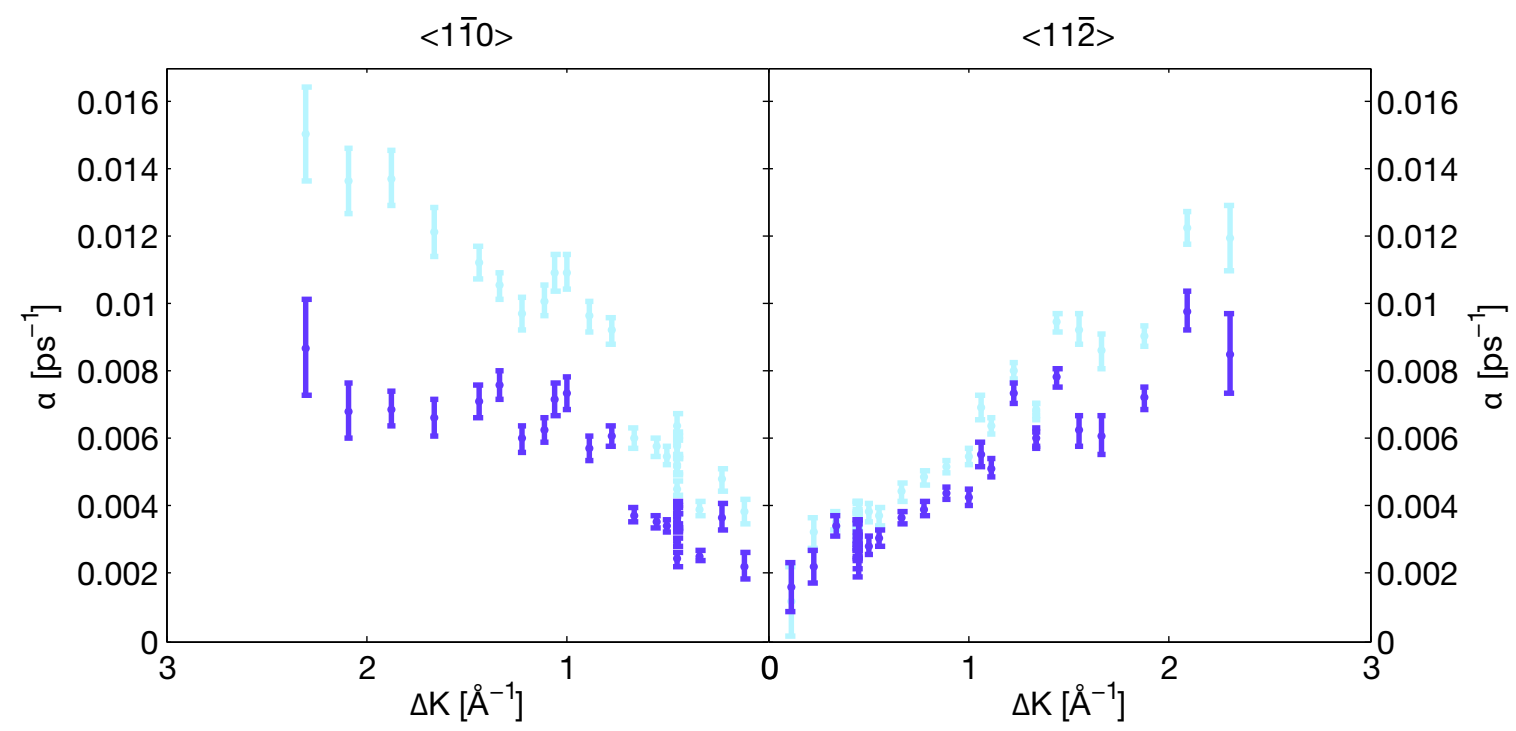

Figure 7.10: $\alpha(\Delta \boldsymbol{K})$ dependence of the diffusive motion of thiophene $/ \mathrm{Cu}(111)$ at $110 \mathrm{~K}$ along two azimuths, $<1 \overline{1} 0>$ and $<11 \overline{2}>$. For a coverage corresponding to $\mathrm{I}_{0} / 3$ $(0.015 \mathrm{ML})$, the dephasing rates for a single exponential starting at $t=25 \mathrm{ps}$ and $t=0.5 \mathrm{ps}$ are shown in dark and light blue, respectively.

Finally, the coverage dependence of the diffusive motion of thiophene has been investigated. Comparing the data for an attenuation level of $\mathrm{I}_{0} / 3$ (0.015 ML) and $\mathrm{I}_{0} / 9(0.022 \mathrm{ML})$ shown in Figure 7.9 , a small increase in jump rate with coverage is 
observed. To investigate the coverage dependence further, measurements of the dephasing rate as a function of coverage were performed at $160 \mathrm{~K}$ and fixed momentum transfer values of $0.4 \AA^{-1}$ and $0.7 \AA^{-1}$, shown in Figure 7.11 as blue and green data points, respectively. Both curves rise continuously with coverage until approximately 0.035 ML, where saturation of the adsorbed thiophene is reached. The curve at $0.7 \AA^{-1}$ rises linearly, while the $0.4 \AA^{-1}$ curve shows a deviation from linear due to its position near the de Gennes dip. Overall, the jump diffusion of thiophene increases approximately linearly with coverage at $160 \mathrm{~K}$, for coverages in the $\alpha$-state described by Milligan et al. [138].

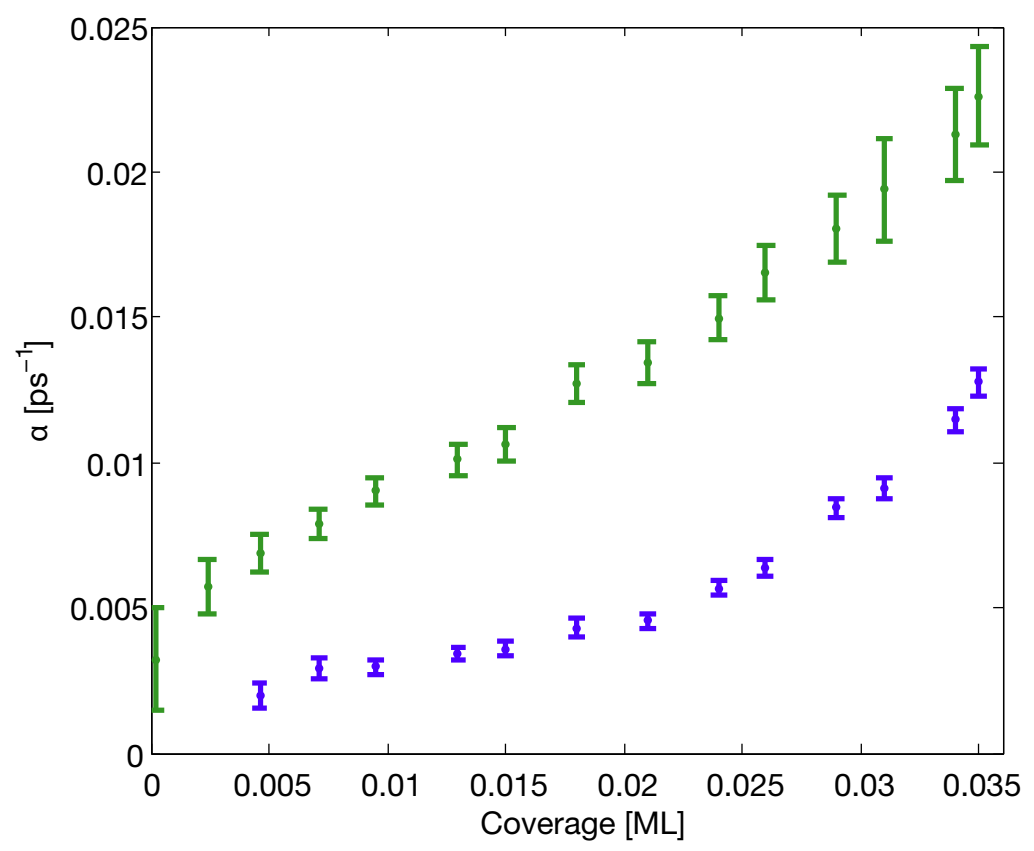

Figure 7.11: Dephasing rate for jump diffusion of thiophene $/ \mathrm{Cu}(111)$ at $160 \mathrm{~K}$ along $<11 \overline{2}>$ as a function of coverage. Blue and green dots show data measured at $0.4 \AA^{-1}$ and $0.7 \AA^{-1}$, respectively. The coverage was calibrated using the average scattering cross section determined from the position of the de Gennes features in Figure 7.9.

To investigate the diffusive motion of thiophene in the $\beta$-state, measurements need to be performed at a lower temperature since desorption of the $\beta$-peak starts below $160 \mathrm{~K}$, e.g. $150 \mathrm{~K}$ for the $\mathrm{I}_{0} / 4000$ curve in Figure 7.4. Figure 7.12 shows the $\alpha(\Delta \boldsymbol{K})$ dependence of thiophene $/ \mathrm{Cu}(111)$ at $140 \mathrm{~K}$, at a coverage corresponding to a specular attenuation of $\mathrm{I}_{0} / 30$, where molecules in the $\alpha$ - and $\beta$-state co-exist (cf. uptake curve in Figure 7.4). Once again, the desphasing rate for an exponential starting at $t=25 \mathrm{ps}$ and $t=0.5 \mathrm{ps}$ is shown, the latter including the contribution from rotation to the ISFs. Due to the lower temperature, the contribution from rotation in the ISFs is increased relative to the $160 \mathrm{~K}$ data, making it more difficult to isolate jump diffusion. Thus, the 
magnitude of the $\alpha(\Delta \boldsymbol{K})$ curve is likely influenced by the signature of rotation. However, the curve shows an approximately sinusoidal $\alpha(\Delta \boldsymbol{K})$ dependence of a shape comparable to that at $160 \mathrm{~K}$ (cf. Figure 7.9), indicating that thiophene diffuses by hopping between top sites even at higher coverage.

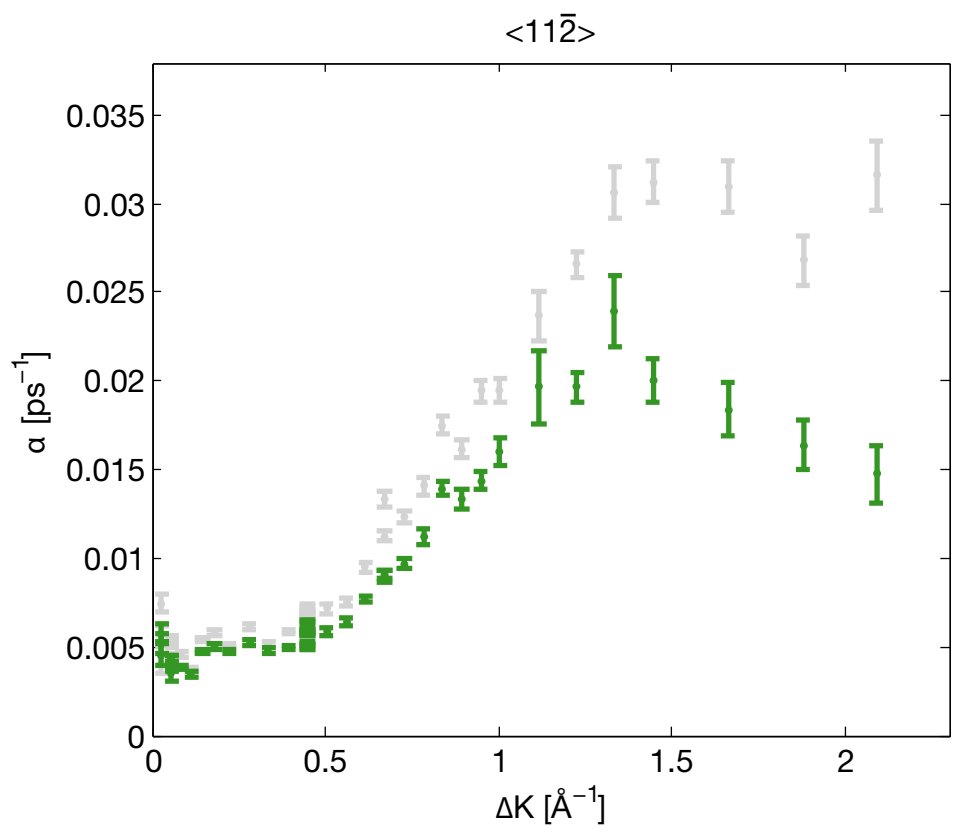

Figure 7.12: $\alpha(\Delta \boldsymbol{K})$ dependence of the diffusive motion of thiophene $/ \mathrm{Cu}(111)$ at $140 \mathrm{~K}$ along $<11 \overline{2}>$ for a coverage corresponding to a specular attenuation of $\mathrm{I}_{0} / 30$. The dephasing rates for a single exponential starting at $t=25 \mathrm{ps}$ and $t=0.5 \mathrm{ps}$ are shown in green and grey, respectively.

The main difference between the results shown in Figures 7.9 and 7.12 is that the $\mathrm{I}_{0} / 30$ curve does not tend to zero for small $\Delta \boldsymbol{K}$ but rather levels off to a constant dephasing rate of $\sim 0.005 \mathrm{ps}^{-1}$. Such behaviour is characteristic for motion vertical to the surface (z-motion) or confined diffusion [6]. In order to study the decay at very small $\Delta \boldsymbol{K}$, temperature dependence measurements at this coverage were performed between $105 \mathrm{~K}$ and $145 \mathrm{~K}$. If the constant level at small $\Delta \boldsymbol{K}$ was due to rotation - a kind of confined diffusion - one would expect the same activation barrier both at small and large $\Delta \boldsymbol{K}$. If it was coupled to jump diffusion, on the other hand, the activation energy at small $\Delta \boldsymbol{K}$ should be equal to that for diffusive motion (cf. Figure 7.8) [99,100]. Two Arrhenius plots for a coverage of $\mathrm{I}_{0} / 30$ at $0.05 \AA^{-1}$ and $0.7 \AA^{-1}$ are shown in Figure 7.13. The apparent activation barrier at large momentum transfers is $32 \pm 2 \mathrm{meV}$, while the process at small momentum transfers shows an activation energy of $11 \pm 2 \mathrm{meV}$. Compared to the energy barriers at equivalent temperatures at lower coverages (cf. weakly activated 
curves in Figure 7.8), the barrier at $0.7 \AA^{-1}$ is slightly increased at the high coverage, suggesting a coverage dependent activation barrier for rotation. More importantly, however, the activation barrier at the high coverage changes with momentum transfer, and the activation energy at low $\Delta \boldsymbol{K}$ is also vastly different from that for jump diffusion. This result implies that the process causing non-zero decay at zero momentum transfer is neither rotation nor coupled to jump diffusion. Instead, it might be due to z-motion of the adsorbate molecules, such as for example a flipping between different tilt angles with respect to the surface.

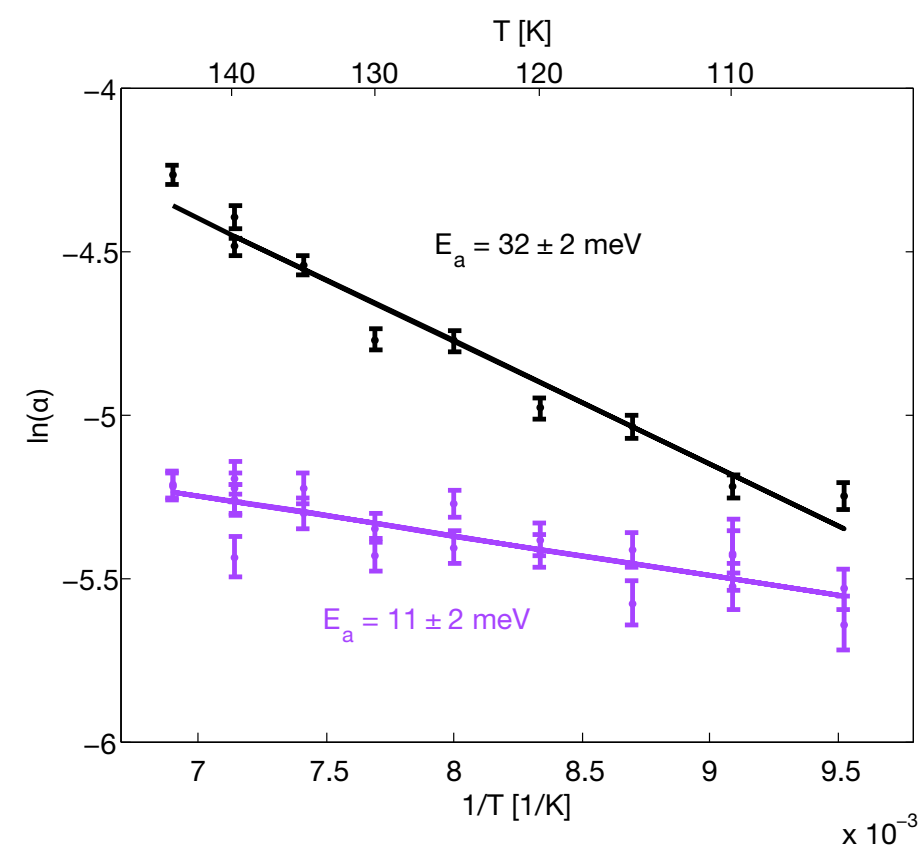

Figure 7.13: Arrhenius plots for a high coverage $\left(\mathrm{I}_{0} / 30\right)$ of thiophene/Cu(111) at $0.05 \AA^{-1}$ and $0.7 \AA^{-1}$ are shown in purple and black, respectively.

\subsection{First principles density functional theory calculations}

As for Cp and pyrrole, the experimental results have been complemented by first principles density functional theory (DFT) calculations for thiophene/ $\mathrm{Cu}(111)$ performed by Sacchi and Jenkins from the Department of Chemistry at Cambridge, which are summarised in this section [95]. 
Adsorption energies for thiophene adsorption on several high symmetry sites on $\mathrm{Cu}(111)$ were calculated using the CASTEP package with the Perdew-Burke-Ernzerhof (PBE) functional [103]. Due to the large contribution of van der Waals forces to the thiophene-surface bond, the dispersion force correction method by Tkatchenko and Scheffler [133] was employed. These calculations confirm Milligan et al.'s prior results [138], giving a preferred adsorption site for a geometry with the sulfur atom on top sites as well as showing an increase in the tilt angle between thiophene and the substrate with coverage. Calculating the adsorption energy for a range of adsorption sites, each for several rotational orientations of the thiophene molecule, DFT predicts an energy barrier to rotation of approximately $20 \mathrm{meV}$ to $30 \mathrm{meV}$ and a translational barrier over hollow sites on the order of $40 \mathrm{meV}$ - in good agreement with the apparent Arrhenius activation energies from HeSE experiments $(59 \pm 4 \mathrm{meV})$.

Calculations of the work function change and dipole moment of thiophene/ $\mathrm{Cu}(111)$ are summarised in Table 7.1. Using the Topping model (cf. Section 5.5), these results provide a handle on the low coverage limit dipole moment, $p_{0}$, and polarisability, $\alpha_{p}$, which were determined as $p_{0}=3.54$ Debye and $\alpha_{p}=74.1 \AA^{3}$ for thiophene $/ \mathrm{Cu}(111)$. These parameters can be used to define the lateral adsorbate interactions in molecular dynamics simulations (cf. Section 7.6).

\begin{tabular}{cccc}
\hline structure & $\boldsymbol{\Theta}[\mathrm{ML}]$ & $\boldsymbol{\Delta} \boldsymbol{\Phi}[\mathrm{eV}]$ & $\boldsymbol{p}[$ Debye $]$ \\
\hline$(2 \sqrt{3} \mathrm{x} 2 \sqrt{3}) \mathrm{R} 30^{\circ}$ & 0.08 & -0.898 & 1.63 \\
$(\sqrt{7} \mathrm{x} \sqrt{7}) \mathrm{R} 19.1^{\circ}$ & 0.14 & -0.917 & 0.97 \\
\hline
\end{tabular}

Table 7.1: Work function change and dipole moment of thiophene/ $\mathrm{Cu}(111)$ from first principles DFT calculations by Sacchi and Jenkins [95].

\subsection{Molecular dynamics simulations reveal an exceptionally high friction}

The centre-of-mass motion of the diffusion of thiophene/ $\mathrm{Cu}(111)$ is modelled in classical Langevin molecular dynamics (MD) simulations (cf. Section 2.4.3). Thiophene is described as a point particle of mass 84 amu moving over a potential energy surface (PES), $V\left(\boldsymbol{r}_{i}\right)$. For jump diffusion between top sites, a PES with minima in a hexagonal arrangement, 
reflecting the geometry of a $\mathrm{Cu}(111)$ surface, and a barrier for diffusion over bridge sites is required. A method analogous to that presented in Sections 5.6 and 6.6 was employed, using a Fourier series to describe the periodicity of the lattice [43,44]. There are two key optimisation parameters - the barrier height and the curvature of the adsorption well which can be altered by raising the normalised PES to a power, $p$. Dipolar interaction forces between the adsorbates are defined by $p_{0}$ and $\alpha_{p}$, estimated from DFT calculations (cf. Section 7.5). A friction coefficient, $\eta$, describes the adsorbate-substrate interactions and a random impulse term ensures energy conservation in the system.

These three parameters - friction, activation barrier and curvature of the adsorption well - were optimised to reproduce the Arrhenius barrier for diffusive motion ( $59 \pm 4 \mathrm{meV})$ and the experimental $\alpha(\Delta \boldsymbol{K})$ curves. Note that rotational motion is not included in the simulations and therefore only the high temperature experimental data is investigated here.

A PES optimised to reproduce the HeSE experiments for thiophene/ $\mathrm{Cu}(111)$ is shown in Figure 7.14. A top view illustrates the hexagonal arrangement of the top sites while a cross-sectional view from bridge to top site along the $<1 \overline{1} 0>$ azimuth (blue) and from top to bridge site via a hollow site along $<11 \overline{2}>$ (red) shows the activation barrier between top and bridge sites as $75 \pm 3 \mathrm{meV}$. The barrier in the PES is larger than the effective Arrhenius activation energy from temperature dependent measurements [44]. The curvature of the adsorption well is that of a simple single Fourier component PES with a power of $p=1.0 \pm 0.1$.

At the start of each simulation, 250 particles with a random kinetic energy in all directions are randomly positioned and allowed to equilibrate for 2000 steps. The ISFs are averaged over 64 runs, each 480 ps long and calculated from the adsorbate trajectories in $0.16 \mathrm{ps}$ time steps. The $\alpha(\Delta \boldsymbol{K})$ curves from simulations best reproducing the experimental data are shown in Figure 7.15, compared with the experimental data. Very good agreement between simulation and experiment is found along both azimuths and for both coverages, 0.015 ML (blue) and 0.022 ML (red). A small deviation can be observerd at low $\Delta \boldsymbol{K}$, where the de Gennes feature indicates the presence of lateral repulsive interactions, and around $1 \AA^{-1}$ along $<11 \overline{2}>$, which could be interpreted as a second order de Gennes feature in the simulations. While the general behaviour observed in experiments in the low momentum transfer region is qualitatively reproduced by the simulations, the exact shape and size of the de Gennes feature is not replicated precisely, suggesting that dipolar pair-wise interaction forces from first principles calculations do not 

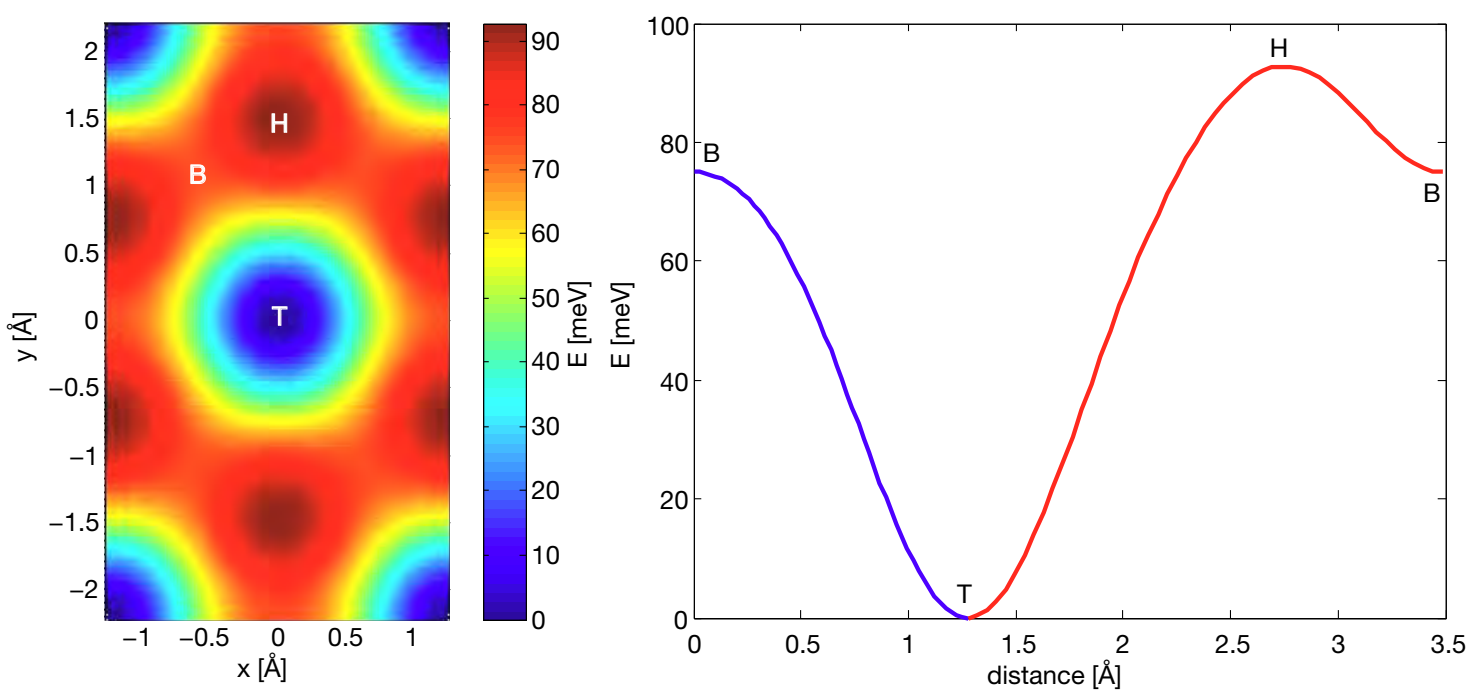

Figure 7.14: Potential energy landscape for thiophene $/ \mathrm{Cu}(111)$ optimised using $\mathrm{MD}$ simulations. A top view is shown on the left, while the right panel shows a cross section from bridge to top site along $<1 \overline{1} 0>$ (blue) and from top to hollow and bridge site along $<11 \overline{2}>$ (red).

fully describe the repulsive interactions between thiophene adsorbates (cf. Section 6.6). The apparent activation energy obtained from simulations at different temperatures is about $59 \pm 4 \mathrm{meV}$, in good agreement with experimental values $(59 \pm 4 \mathrm{meV})$.

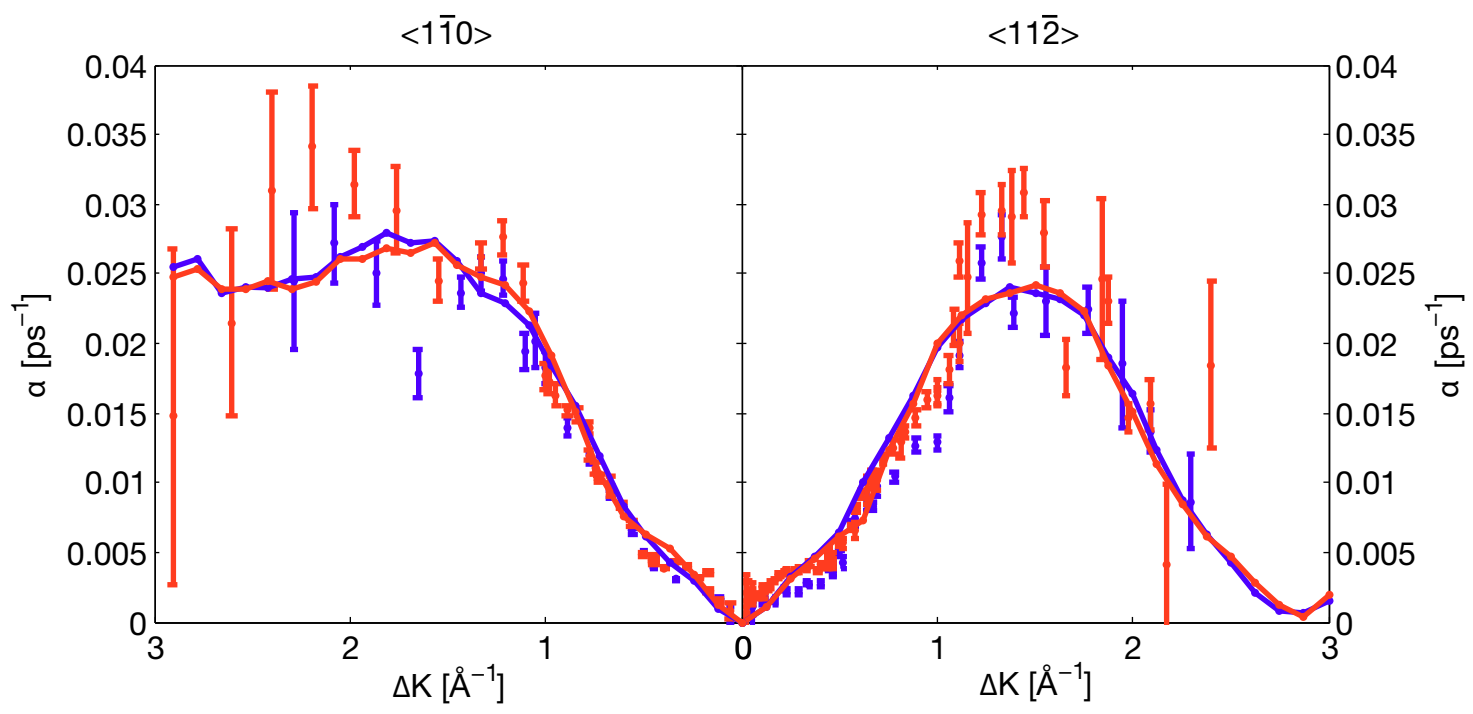

Figure 7.15: $\alpha(\Delta \boldsymbol{K})$ curves from MD results for 0.015 ML (blue) and 0.022 ML (red) thiophene/ $\mathrm{Cu}(111)$, compared with HeSE data for the same coverages (shown as dots in corresponding colours). Good agreement is found at both coverages and along the two principal crystal directions. 
The friction coefficient for the simulations shown in Figure 7.15, $\eta=5 \mathrm{ps}^{-1}$, is exceptionally high. At such a high friction, single jumps dominate the diffusion behaviour of thiophene, as illustrated by the trajectories shown in Figure 7.16 (A) where only a negligible number of long jumps are observed. In general, friction changes the distribution of jump lengths and thereby the shape of the $\alpha(\Delta \boldsymbol{K})$ curve [44]. In this particular high friction case, however, a variation in friction only leads to a change in the height of the curve, i.e. the jump frequency, but not its shape. Within the error bars of the Arrhenius activation energy from experiments the change in height can be counterbalanced by the barrier height in the PES (indicated by the error bars given above), making it difficult to determine an accurate value for the friction. Probing a wide parameter range, the friction coefficient has been narrowed down to $\eta=5 \pm 2 \mathrm{ps}^{-1}$. These error bars are significantly larger than those for the friction of $\mathrm{Cp}$ or pyrrole on $\mathrm{Cu}(111)$, reflecting the trade-off between two parameters.
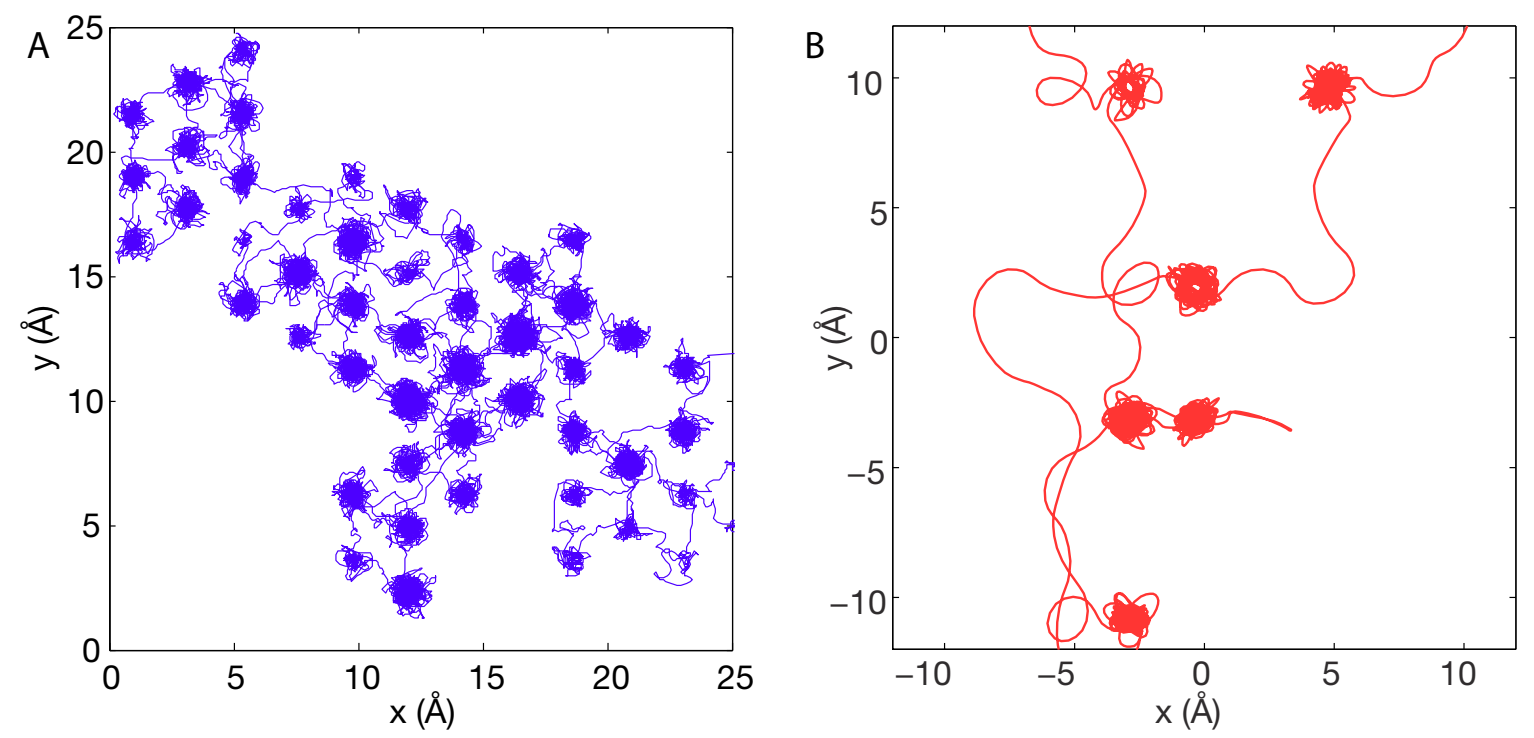

Figure 7.16: Trajectories for $\mathrm{MD}$ simulations for (A) thiophene $/ \mathrm{Cu}(111)$ hopping between adjacent top sites, compared with those for (B) $\mathrm{Na} / \mathrm{Cu}(100)$, a low friction system (by Alexandrowicz et al. [99]).

For a more accurate determination of the friction, an alternative approach such as that using the intracell diffusion described in Section 5.9 would be required. Unfortunately, in this particular instance the signature for intracell diffusion is difficult to analyse as the fast drop in the ISFs is very weak and cannot be separated easily from the decays caused by rotation. Independent of the precise value, however, the lower bound for the friction coefficient, at approximately $3 \mathrm{ps}^{-1}$, indicates that the friction for thiophene is 
exceptionally large. Table 7.2 compares the friction coefficients for a range of different adsorbate systems, demonstrating the trend towards larger friction with increasing molecule size. To illustrate the difference between atomic and molecular systems, the trajectories for $\mathrm{Na} / \mathrm{Cu}(100)$ [99] are reproduced in Figure $7.16(\mathrm{~B})$, showing a much larger contribution of long jumps than thiophene/ $\mathrm{Cu}(111)$ (cf. Figure $7.16(\mathrm{~A})$ ).

\begin{tabular}{lll}
\hline Adsorbate system & Friction $\left[\mathbf{p s}^{-\mathbf{1}}\right]$ & Reference \\
\hline $\mathrm{Na} / \mathrm{Cu}(100)$ & 0.5 & {$[99]$} \\
$\mathrm{K} / \mathrm{Cu}(100)$ & 0.22 & {$[100]$} \\
$\mathrm{Cs} / \mathrm{Cu}(100)$ & 0.025 & {$[31]$} \\
$\mathrm{CO} / \mathrm{Cu}(100)$ & $0.1-0.5$ & {$[149]$} \\
$\mathrm{CO} / \mathrm{Cu}(111)$ & 0.8 & {$[44]$} \\
$\mathrm{CO} / \mathrm{Pt}(111)$ & 0.7 & {$[43]$} \\
Propane/Pt(111) & $0.8 \pm 0.2$ & {$[112]$} \\
Benzene/Graphite & 2.2 & {$[8]$} \\
$\mathrm{Cp} / \mathrm{Cu}(111)$ & $2.5 \pm 0.5$ & Chapter 5 \\
Pyrrole/Cu(111) & $2.0 \pm 0.4$ & Chapter 6 \\
Thiophene/Cu(111) & $5 \pm 2$ & Chapter 7 \\
\hline
\end{tabular}

Table 7.2: Friction coefficients for a variety of adsorbate systems, ranging from atomic to molecular species.

Intuitively, the increase in friction with adsorbate size might be understood in terms of an increased contact area between adsorbate and substrate. The present work, however, demonstrates that the actual process is in fact more complicated. Thiophene, bonding predominantly through the sulfur atom and with the carbon atoms tilted away from the surface, shows the highest friction of all aromatic molecules studied, yet it should have a smaller effective contact area than $\mathrm{Cp}$ or pyrrole on the same substrate. An alternative explanation for increased friction can be found in the work of de Wijn and Fasolino $[150,151]$. Their simulations of benzene on a graphite surface show a large friction value, in good agreement with spin-echo experiments [8], and predict a large contribution to the friction from the degrees of freedom of the molecule itself - an effect independent of the contact area between adsorbate and substrate. It is therefore conceivable that the exceptionally high friction of thiophene $/ \mathrm{Cu}(111)$ is due to the low frequency of its external vibrational mode perpendicular to the surface that involves only bending of the $\mathrm{S}-\mathrm{Cu}$ bond but no change in bond length. Thiophene/ $\mathrm{Cu}(111)$ is thus an 
ideal model system to investigate microscopic aspects of friction in molecular adsorbates and further work in a collaboration with de Wijn and Fasolino is under way.

\subsection{Conclusions}

The dynamics of thiophene/ $\mathrm{Cu}(111)$ were investigated using a combined HeSE and DFT study. TDS measurements at low coverage show good agreement with the results by Milligan et al. [138] and provide a means of relating the coverages used in the dynamics measurements to the different adsorption regimes described in the literature [138].

HeSE measurements of the thiophene/Cu(111) dynamics exhibit multi-component lineshapes, while temperature dependent measurements reveal a kink in the Arrhenius plot, implying that two competing activated processes occur. The more strongly activated process, dominating at temperatures above $145 \mathrm{~K}$, was identified as jump diffusion, while comparison with ethanethiolate $/ \mathrm{Cu}(111)$ [132] suggests rotation of the molecule around a $\mathrm{S}-\mathrm{Cu}$ anchor point dominates at lower temperatures, exhibiting an apparent activation energy of $22 \pm 2 \mathrm{meV}$. First principles DFT calculations by Sacchi and Jenkins confirm that thiophene can perform jump diffusion over an energy barrier on the order of $40 \mathrm{meV}$, as well as rotational motion showing an activation energy between $20 \mathrm{meV}$ and $30 \mathrm{meV}$ [95]. In addition, experiments at a higher coverage provide evidence for motion perpendicular to the surface.

The diffusive motion of thiophene exhibits single jumps between adjacent top sites over an apparent activation barrier of $59 \pm 4 \mathrm{meV}$. MD simulations give a barrier in the potential energy landscape of $75 \pm 3 \mathrm{meV}$ and an exceptionally high friction coefficient of $5 \pm 2 \mathrm{ps}^{-1}$. These results suggest that the large friction commonly observed for extended molecular adsorbates is not simply due to an increased contact area between adsorbate and substrate, which supports previous work by de Wijn and Fasolino suggesting the degrees of freedom of the adsorbed molecule as a cause of increased friction. 


\section{Chapter 8}

\section{Conclusions}

The present work is motivated by the desire to extend the field of application for helium-3 spin-echo (HeSE) spectroscopy from atomic and small molecular adsorbates to more complex surface dynamical systems. This objective has been addressed with a twofold approach. Firstly, improvements were made to the experimental equipment with the construction of MiniScat, a new helium atom scattering apparatus, as well as an improved helium beam source. Secondly, a series of related five-membered aromatic adsorbates on the hexagonal $\mathrm{Cu}(111)$ surface were investigated to study the surface dynamics of these prototypical organic molecules and to ascertain the degree to which the existing theoretical framework extends to molecular adsorbates.

\subsection{Equipment development}

The design and construction of MiniScat was presented in Chapter 3. Characterisation measurements have demonstrated the performance of the new instrument. Due to the uniqueness of the HeSE spectrometer, measurement time is limited and the careful preparation and scheduling of experiments is required. MiniScat now provides a facility for sample preparation and equipment development purposes, while also acting as a standalone spectrometer for structural studies that cannot be performed on the HeSE machine, such as thermal desorption studies. Since the study of more complex systems routinely creates a need for extensive preliminary work to investigate the adsorption behaviour before the actual dynamics measurements can be performed, MiniScat is a crucial stepping stone towards the aim of the present thesis and has already been in productive use in the Surface Physics Group for the past two years. 
Improvements of the HeSE spectrometer itself were achieved by developing a new helium beam source, as presented in Chapter 4. The source is one of the main components of the instrument and determines the energy resolution of the experiment through the helium beam energy. Due to a tendency of complex molecular adsorbates to move more slowly than atomic species, a need for improved resolution arises with the investigation of increasingly complex adsorbate systems. The new high intensity helium beam source can operate at beam energies as low as $2.5 \mathrm{meV}$, improving the resolution of the HeSE spectrometer by a factor of 5.5 and extending the measurement range from a previously longest accessible spin-echo time of 680 ps to 3.8 ns. Thus, the new source opens up a whole new measurement range, making the investigation of slowly diffusing adsorbates possible. This significant improvement has been demonstrated in first measurements studying the diffusion of benzene/ $\mathrm{Cu}(100)$ performed by Holly Hedgeland [69].

Furthermore, an investigation of the effect of shape and size of the skimmer and its mount revealed two competing attenuation processes on either side of the skimmer. The new design, reducing the size of components in immediate vicinity of the skimmer, increased the intensity of the helium beam by approximately $20 \%$. Further improvements of the performance of the source are conceivable by replacing the current skimmer with one of a wider angle to study the attenuation within the skimmer itself.

\subsection{Dynamics experiments}

Moving on to the experimental part of this thesis, Chapters 5, 6 and 7 presented a study of the diffusive motion of three five-membered aromatic molecules, specifically cyclopentadienyl (Cp), pyrrole and thiophene on $\mathrm{Cu}(111)$. All three molecules were found to be highly mobile on the hexagonal surface and move in jump diffusion between adjacent adsorption sites.

The lineshapes in the HeSE data are of a complex nature, reflecting contributions from several different processes. In the case of $\mathrm{Cp}$, vibrational motion of the copper substrate and the molecule itself could be identified, as well as a fast drop at small spin-echo times, corresponding to rapid diffusive motion within the adsorption well. The fast decay signature for intracell motion had been predicted using molecular dynamics simulations [98], yet little experimental evidence confirming the theoretical work has been available until now. Similar fast decay signatures were also observed in the lineshapes of pyrrole and thiophene data, indicating that diffusive motion on short timescales is 
comparable for all three adsorbate species. In the case of thiophene, the fast decay signature proved difficult to analyse due to the presence of rotational motion, highlighting the value of a comparison with similar systems such as $\mathrm{Cp} / \mathrm{Cu}(111)$, which enabled the identification of these features as intracell motion.

On longer spin-echo times the decays from jump diffusion dominate the measured data. Investigating the $\alpha(\Delta \boldsymbol{K})$ dependence and the lineshapes in the HeSE data showed that the three adsorbates each prefer different adsorption sites: Cp hops between fcc and hcp hollow sites, while pyrrole prefers bridge sites and thiophene adsorbs on top of the copper atoms. On an fcc(111) surface, top sites form a simple Bravais lattice, evident as a single decay in the intermediate scattering function (ISF), whereas multiple sites in the unit cell result in a sum of two exponentials in the ISF in the case of hollow or bridge sites. For $\mathrm{Cp} / \mathrm{Cu}(111)$, the two decays for hopping between fcc and hcp sites could be distinguished clearly, providing a first experimental confirmation of the predicted multi-component lineshapes. In addition, the double decays are accessible to comprehensive Bayesian statistical analysis probing the probability space across the entire data set to determine the energy difference between the hollow sites: $12.3 \pm 0.3 \mathrm{meV}$. In the case of pyrrole/ $\mathrm{Cu}(111)$, however, strongly repulsive lateral interactions between the adsorbates alter the experimental lineshapes, changing their appearance to a single exponential. Molecular dynamics (MD) simulations comparing the behaviour of a single particle with that of multiple interacting adsorbates confirmed the effect of lateral interactions on the lineshapes, as predicted in previous work by Martínez-Casado [34]. Finally, measurements of the motion of thiophene revealed complex lineshapes due to the presence of two competing processes, jump diffusion as well as rotational motion around a $\mathrm{S}-\mathrm{Cu}$ anchor point.

The observation of a distinct preferred adsorption site for each system is in accordance with the different types of bonding to the substrate. DFT calculations by Sacchi and Jenkins $[95,101,102]$ showed that Cp, which is not chemically stable in the gas phase, receives 1.1 electrons from the substrate upon adsorption, forming an ionic bond. Pyrrole, on the other hand, is a neutral molecule in the gas phase and forms a weak bond with the copper surface, predominantly through van der Waals interactions. Thiophene, finally, has a lone electron pair on the sulfur atom that is not delocalised in the aromatic ring and thus free to bond to the surface [138]. Therefore, thiophene adopts a tilted geometry with a covalent $\mathrm{S}-\mathrm{Cu}$ bond and weak dispersion forces between the carbon atoms and the substrate. 
Despite differences in the vertical bond strength, experiments revealed apparent activation energies for horizontal motion that are fairly similar for all three adsorbates: $41 \pm 1 \mathrm{meV}$ for $\mathrm{Cp}, 53 \pm 4 \mathrm{meV}$ for pyrrole and $59 \pm 4 \mathrm{meV}$ for thiophene. DFT calculations by Sacchi and Jenkins demonstrated that the apparent activation energy for pyrrole is largely due to a site dependence in the zero point energies (ZPE) of the vibrational modes of the molecule. Interestingly, the largest contribution to the ZPE difference is made by the $\mathrm{C}-\mathrm{H}$ and $\mathrm{N}-\mathrm{H}$ out-of-plane bending and ring torsion modes that are not directly involved in the diffusion. Further HeSE measurements of deuterated pyrrole $/ \mathrm{Cu}(111)$ could be performed in order to study the effect of quantum modes experimentally, with an expected result of an altered activation barrier for diffusion.

The centre-of-mass motion of the five-membered molecules on $\mathrm{Cu}(111)$ was modelled using classical Langevin MD simulations describing the adsorbates as a point particle moving across a potential energy landscape. By optimising friction, activation barrier and the shape of the adsorption well, the experimental results were reproduced very well. The simulations have yielded further information on microscopic aspects of the diffusive motion, showing a large friction coefficient for each of the three systems: $2.5 \pm 0.5 \mathrm{ps}^{-1}$ for $\mathrm{Cp}, 2.0 \pm 0.4 \mathrm{ps}^{-1}$ for pyrrole and $5 \pm 2 \mathrm{ps}^{-1}$ for thiophene on $\mathrm{Cu}(111)$. Such exceptionally high friction for thiophene, which adsorbs tilted at an angle to the substrate, indicates that the increase in friction commonly observed for molecular adsorbates cannot be explained simply by an increase of the adsorbate-substrate contact area. Prior work by de Wijn and Fasolino $[150,151]$ provides an alternative explanation, where the degrees of freedom of the molecules make a large contribution to the friction [150,151]. Joint work is currently ongoing, aiming to investigate the microscopic aspects of friction in molecular adsorbate systems in collaboration with the aforementioned researchers.

Lastly, MD simulations have demonstrated that a simple $1 / r^{3}$ dipole-dipole potential cannot adequately describe the lateral interactions between strongly interacting pyrrole adsorbates, as apparent in the deviation of the simulated de Gennes features from experimental results. Simulations testing a new interaction potential that accounts for the extended nature of the molecule have demonstrated the need for further theoretical work looking into the precise functional form of the interaction potential.

Overall, the present work has laid a foundation for the study of complex surface dynamical systems, providing measurements of the motion of three prototypical adsorbates as well as improved experimental equipment, which will hopefully be useful for future work in this field. 


\section{Bibliography}

[1] R. Gomer, Diffusion of adsorbates on metal surfaces, Rep. Prog. Phys., 53, 917-1002 (1990).

[2] J. V. Barth, G. Costantini and K. Kern, Engineering atomic and molecular nanostructures at surfaces, Nature, 437, 671 (2005).

[3] G. A. Somorjai, Introduction to Surface Chemistry and Catalysis, John Wiley \& Sons (1994).

[4] J. V. Barth, Transport of adsorbates at metal surfaces: from thermal migration to hot precursors, Surf. Sci. Rep., 40, 3-5, 75-149 (2000).

[5] T. Ala-Nissila, R. Ferrando and S. C. Ying, Collective and single particle diffusion on surfaces, Advances in Physics, 51, 3, 949 (2002).

[6] A. P. Jardine, H. Hedgeland, G. Alexandrowicz, W. Allison and J. Ellis, Helium-3 spin-echo: Principles and application to dynamics at surfaces, Prog. Surf. Sci., 84, 323 (2009).

[7] M. J. Bée, Quasielastic neutron scattering, Hilger, Bristol (1988).

[8] H. Hedgeland, P. Fouquet, A. P. Jardine, G. Alexandrowicz, W. Allison and J. Ellis, Measurement of single-molecule frictional dissipation in a prototypical nanoscale system, Nature Phys., 5, 561 (2009).

[9] E. Hulpke (Editor), Helium atom scattering from surfaces, Springer-Verlag (1992).

[10] A. P. Graham, The low energy dynamics of adsorbates on metal surfaces investigated with helium atom scattering, Surf. Sci. Rep., 49, 115-168 (2003).

[11] A. P. Jardine, J. Ellis and W. Allison, Quasi-elastic helium-atom scattering from surfaces: experiment and interpretation, J. Phys.: Condens. Matter, 14, 6173-6191 (2002). 
[12] S. Klyatskaya, F. Klappenberger, U. Schlickum, D. Kühne, M. Marschall, J. Reichert, R. Decker, W. Krenner, G. Zoppellaro, H. Brune, J. V. Barth and M. Ruben, Surface-Confined Self-Assembly of Di-carbonitrile Polyphenyls, Adv. Funct. Mater., 21, 1230-1240 (2011).

[13] A. P. Jardine, S. Dworski, P. Fouquet, G. Alexandrowicz, D. J. Riley, G. Y. H. Lee, J. Ellis and W. Allison, Ultrahigh-resolution spin-echo measurements of surface potential energy landscapes, Science, 304, 1790 (2004).

[14] A. P. Jardine, G. Alexandrowicz, H. Hedgeland, W. Allison and J. Ellis, Studying the microscopic nature of diffusion with helium-3 spin-echo, Phys. Chem. Chem. Phys., 11, 3355 (2009).

[15] D. Farías and K.-H. Rieder, Atomic beam diffraction from solid surfaces, Rep. Prog. Phys., 61, 1575-1664 (1998).

[16] G. Alexandrowicz and A. P. Jardine, Helium spin-echo spectroscopy: studying surface dynamics with ultra-high-energy resolution, J. Phys.: Condens. Matter, 19, 305001 (2007).

[17] L. Van Hove, Correlations in Space and Time and Born Approximation Scattering in Systems of Interacting Particles, Phys. Rev., 95, 1, 249-262 (1954).

[18] G. Vineyard, Scattering of Slow Neutrons by a Liquid, Phys. Rev., 110, 5, 999-1010 (1958).

[19] J. W. M. Frenken, B. J. Hinch and J. P. Toennies, He scattering study of diffusion at a melting surface, Surf. Sci., 211/212, 21 (1989).

[20] M. DeKieviet, D. Dubbers, C. Schmidt, D. Scholz and U. Spinola, ${ }^{3}$ He spin echo: New atomic beam technique for probing phenomena in the neV range, Phys. Rev. Lett., 75, 1919-1922 (1995).

[21] M. DeKieviet, D. Dubbers, M. Klein, C. Schmidt and M. Skrzipczyk, Surface science using molecular beam spin echo, Surf. Sci., 377-379, 112-117 (1997).

[22] F. Mezei, Neutron spin echo: A new concept in polarized thermal neutron techniques, Z. Physik, 255, 146 (1972).

[23] P. Fouquet, A. P. Jardine, S. Dworski, G. Alexandrowicz, W. Allison and J. Ellis, Thermal energy ${ }^{3} \mathrm{He}$ spin-echo spectrometer for ultrahigh resolution surface dynamics measurements, Rev. Sci. Instrum., 76, 53109 (2005). 
[24] H. Hedgeland, A. P. Jardine, W. Allison and J. Ellis, Anomalous attenuation at low temperatures in a high intensity helium beam source, Rev. Sci. Instrum., 76, $123111(2005)$.

[25] H. Hedgeland, P. R. Kole, W. Allison, J. Ellis and A. P. Jardine, An improved high intensity recycling helium-3 beam source, Rev. Sci. Instrum., 80, 76110 (2009).

[26] A. P. Jardine, P. Fouquet, J. Ellis and W. Allison, Hexapole magnet system for thermal energy ${ }^{3} \mathrm{He}$ atom manipulation, Rev. Sci. Instrum., 72, 10, 3834-3841 (2001).

[27] P. R. Kole, Dynamics and morphology of metal and metal oxide surfaces, Ph.D. thesis, University of Cambridge (2011).

[28] H. Hedgeland, The Development of Quasi-Elastic Helium-3 Spin-Echo Spectroscopy as a Tool for the Study of Surface Dynamics, Ph.D. thesis, University of Cambridge (2006).

[29] A. R. Alderwick, A. P. Jardine, H. Hedgeland, D. A. MacLaren, W. Allison and J. Ellis, Simulation and analysis of solenoidal ion sources, Rev. Sci. Instrum., 79, 123301 (2008).

[30] D. M. Chisnall, To be submitted, Ph.D. thesis, University of Cambridge (2012).

[31] A. P. Jardine, G. Alexandrowicz, H. Hedgeland, R. D. Diehl, W. Allison and J. Ellis, Vibration and diffusion of $\mathrm{Cs}$ atoms on $\mathrm{Cu}(001)$, J. Phys.: Condens. Matter, 19, 305010 (2007).

[32] D. J. Ward, To be submitted, Ph.D. thesis, University of Cambridge (2012).

[33] J. L. Vega, R. Guantes, S. Miret-Artés and D. A. Micha, Collisional line shapes for low frequency vibrations of adsorbates on a metal surface, J. Chem. Phys., 121, 8580 (2004).

[34] R. Martínez-Casado, J. L. Vega, A. S. Sanz and S. Miret-Artés, Line shape broadening in surface diffusion of interacting adsorbates with quasielastic He atom scattering, Phys. Rev. Lett., 98, 216102 (2007).

[35] C. T. Chudley and R. J. Elliot, Neutron Scattering from a Liquid on a Jump Diffusion Model, Proc. Phys. Soc., 77, 2, 353-361 (1960). 
[36] J. M. Rowe, K. Sköld, H. E. Flotow and J. J. Rush, Quasielastic neutron scattering by hydrogen in the $\alpha$ and $\beta$ phases of vanadium hydride, J. Phys. Chem. Solids, 32, 41-54 (1971).

[37] O. G. Randl, B. Sepiol, G. Vogl, R. Feldwisch and K. Schroeder, Quasielastic Mössbauer spectroscopy and quasielastic neutron scattering from non-Bravais lattices with differently occupied sublattices, Phys. Rev. B, 49, 8768 (1994).

[38] F. E. Tuddenham, H. Hedgeland, A. P. Jardine, B. A. J. Lechner, B. J. Hinch and W. Allison, Lineshapes in quasi-elastic scattering from species hopping between non-equivalent surface sites, Surf. Sci., 604, 1459-1475 (2010).

[39] F. E. Tuddenham, Helium atom scattering as a probe of hydrogen adsorption and dynamics, Ph.D. thesis, University of Cambridge (2011).

[40] U. Harten, J. P. Toennies and C. Wöll, Helium time-of-flight spectroscopy of surface-phonon dispersion curves of the noble metals, Faraday Discuss. Chem. Soc., 80, 137 (1985).

[41] P. G. De Gennes, Liquid dynamics and inelastic scattering of neutrons, Physica, 25, 825-839 (1959).

[42] J. Ellis and A. P. Graham, The use of quasielastic helium atom scattering to study correlated motion in adsorbate overlayers, Surf. Sci., 377, 833 (1997).

[43] G. Alexandrowicz, P. R. Kole, E. Y. M. Lee, H. Hedgeland, R. Ferrando, A. P. Jardine, W. Allison and J. Ellis, Observation of uncorrelated microscopic motion in a strongly interacting adsorbate system, J. Am. Chem. Soc., 130, 6789-6794 (2008).

[44] P. R. Kole, A. P. Hedgeland, H. Jardine, W. Allison, J. Ellis and G. Alexandrowicz, Probing the non-pairwise interactions between $\mathrm{CO}$ molecules moving on a $\mathrm{Cu}(111)$ surface, J. Phys.: Condens. Matter, 24, 104016 (2012).

[45] H. A. Kramers, Brownian motion in a field of force and the diffusion model of chemical reactions, Physica, 7, 4, 284 (1940).

[46] R. Zwanzig, Nonequilibrium statistical mechanics, Oxford University Press (2001).

[47] McAllister Technical Services, West 280 Prairie Avenue, Coeur dAlene, ID 83815, USA. http://www.mcallister.com. 
[48] Oerlikon Leybold Vacuum GmbH, Bonner Strasse 498 (Bayenthal), 50968 Cologne, Germany. http://www.oerlikon.com/leyboldvacuum.

[49] Edwards Limited, Manor Royal, Crawley, West Sussex, RH10 9LW, United Kingdom. http://www.edwardsvacuum.com.

[50] VAT Vakuumventile AG, Seelistrasse, 9469 Haag, Switzerland. http://www.vatvalve.com.

[51] Shimadzu Corporation, 3. Kanda Nishikicho 1-chome, Chiyoda-ku, Tokyo, Japan. http://www.shimadzu.com.

[52] Alcatel Vacuum Technology, France; now adixen Vacuum Products by Pfeiffer Vacuum. http://www.adixen.com.

[53] A. P. Jardine, Quasi-elastic Helium Atom Scattering: Interpretation and Instrumentation, Ph.D. thesis, University of Cambridge (2001).

[54] Beam Dynamics, Inc., 13749 Shelter Cove Drive, Jacksonville, FL 32225 USA. http://www.beamdynamicsinc.com.

[55] Pfeiffer Vacuum, Emmeliusstrasse 33, 35614 Asslar, Germany. http://www.pfeiffervacuum.de.

[56] Hiden Analytical 420 Europa Boulevard, Warrington, WA5 7UN, United Kingdom. http://www.hidenanalytical.com.

[57] K. A. Stoerzinger, Nanoscale Dynamics of Benzene and Naphthalene on Graphene/Ru(0001), Master's thesis, University of Cambridge (2011).

[58] J. Zhu, Structure and dynamics of hydrogen on metal surfaces, First Year Ph.D. Report, University of Cambridge (2012).

[59] E. G. McRae and C. W. Caldwell, Low-energy electron diffraction study of lithium fluoride (100) surface, Surf. Sci., 2, 509-515 (1964).

[60] D. R. Miller, Free Jet Sources. In: G. Scoles (Ed.), Atomic and Molecular Beam Methods, 14-53, Oxford University Press (1988).

[61] G. Alexandrowicz, Helium Spin Echo Spectroscopy: Measuring the Dynamics of Atoms, Molecules and Surfaces, Ph.D. thesis, University of Cambridge (2005). 
[62] M. DeKieviet, D. Dubbers, S. Hafner and F. Lang. In: R. Campargue (Ed.), Atomic and Molecular Beams: The State of the Art, 161, Springer (2001).

[63] A. Kantrowitz and J. Grey, A high intensity source for the molecular beam. Part I. Theoretical, Rev. Sci. Instrum., 22, 328 (1951).

[64] R. Campargue, Progress in overexpanded supersonic jets and skimmed molecular beams in free-jet zones of silence, J. Phys. Chem., 88, 4466-4474 (1984).

[65] Sumitomo (SHI) Cryogenics of America, Inc., 1833 Vultee Street, Allentown, PA 18103-4783, USA. http://www.shicryogenics.com (previously APD Cryogenics Inc.).

[66] J. R. Buckland, R. L. Folkerts, R. B. Balsod and W. Allison, A simple nozzle design for high speed-ratio molecular beams, Meas. Sci. Technol., 8, 933-935 (1997).

[67] Lake Shore Cryotronics Inc., 575 McCorkle Blvd, Westerville, OH 43082-8699, USA. http://www.lakeshore.com.

[68] M\&M Materials Ltd., Hibernia Way, Trafford Park, Manchester M32 0ZD, United Kingdom. http://www.apiezon.com.

[69] H. Hedgeland, unpublished data.

[70] A. P. Jardine, private communications.

[71] J. D. Dunitz, L. E. Orgel and A. Rich, The Crystal Structure of Ferrocene, Acta Cryst., 9, 373 (1956).

[72] G. Brizuela and R. Hoffmann, $\mathrm{C}_{5} \mathrm{H}_{5}$ on a $\mathrm{Pt}(111)$ surface: Electronic structure and bonding, J. Phys. Chem. A, 102, 9618 (1998).

[73] G. Szulczewski, S. Sanvito and G. Coey, A spin of their own, Nature Mater., 8, $693(2009)$.

[74] G. Witte, S. Lukas, P. S. Bagus and C. Wöll, Vacuum level alignment at organic/metal junctions: "Cushion" effect and the interface dipole, Appl. Phys. Lett., 87, 263502 (2005).

[75] L. Romaner, G. Heimel, J.-L. Brédas, A. Gerlach, F. Schreiber, R. L. Johnson, J. Zegenhagen, S. Duhm, N. Koch and E. Zojer, Impact of bidirectional charge transfer and molecular distortions on the electronic structure of a metal-organic interface, Phys. Rev. Lett., 99, 256801 (2007). 
[76] A. Haaland, Molecular structure and bonding in the 3d metallocenes, Acc. Chem. Res., 12, 415-422 (1979).

[77] B. E. Applegate, T. A. Miller and T. A. Barckholtz, The Jahn-Teller and related effects in the cyclopentadienyl radical. I. The ab initio calculation of spectroscopically observable parameters, J. Chem. Phys., 114, 11, 4855 (2001).

[78] P. Seiler and J. D. Dunitz, A new interpretation of the disordered crystal structure of ferrocene, Acta Cryst., B35, 1068 (1979).

[79] C.-H. Sun, B. E. Bent and J. G. Chen, Chemistry of cyclopentadiene on a $\mathrm{Cu}(100)$ surface: Detection of cyclopentadienyl $\left(\mathrm{C}_{5} \mathrm{H}_{5}\right)$ species as reaction intermediates, $J$. Vac. Sci. Technol. A, 15, 1581 (1997).

[80] N. R. Avery, Bonding and reactivity of cyclopentene on Pt(111), Surf. Sci., 146, 363-381 (1984).

[81] N. R. Avery, Cyclopentene adsorption on Pt(111): Spectroscopic identification of an adsorbed cyclopentadienyl, Surf. Sci., 137, L109-L116 (1984).

[82] N. R. Avery, Adsorption and reactivity of cyclopentane on Pt(111), Surf. Sci., 163, 357-368 (1985).

[83] N. R. Avery, Adsorption and reactivity of 1-3 cyclopentadiene on $\operatorname{Pt}(111), J$. Electron Spectrosc. Relat. Phenom., 39, 1-9 (1986).

[84] G. Brizuela and N. J. Castellani, $\mathrm{C}_{5} \mathrm{H}_{n}$ rings adsorbed on $\mathrm{Pt}(111)$ : a theoretical study, Surf. Sci., 401, 297 (1998).

[85] G. Brizuela and N. J. Castellani, A theoretical study of dehydrogenation of cyclopentene on Pt(111), J. Mol. Catal. A: Chem., 139, 209-218 (1999).

[86] C. Becker, F. Delecq, J. Breitbach, G. Hamm, D. Franke, F. Jäger and K. Wandelt, Adsorption of Cyclopentene on $\mathrm{Pt}(111)$ and Ordered $\mathrm{Pt}_{n} \mathrm{Sn} / \mathrm{Pt}(111)$ Surface Alloys, J. Phys. Chem. B, 108, 18960-18971 (2004).

[87] N. C. Comelli, M. B. Lopez and E. A. Castro, Theoretical study of the adsorption of $\mathrm{C}_{5} \mathrm{H}_{n}$ on $\mathrm{Ni}(100)$ and $\mathrm{Ni}(111)$ surfaces, J. Mol. Struct., 726, 197 (2005).

[88] E. Germán, S. Simonetti, E. Pronsato, A. Juan and G. Brizuela, $\mathrm{c}_{-} \mathrm{C}_{5} \mathrm{H}_{5}$ on a $\mathrm{Ni}(111)$ surface: Theoretical study of the adsorption, electronic structure and bonding, Appl. Surf. Sci., 254, 5831 (2008). 
[89] E. Germán, I. López-Corral, A. Juan and G. Brizuela, A theoretical study of cyclopentene $\left(\mathrm{c}-\mathrm{C}_{5} \mathrm{H}_{8}\right)$ dehydrogenation to cyclopentadienyl anion $\left(\mathrm{c}-\mathrm{C}_{5} \mathrm{H}_{5}^{-}\right)$on Ni(111), J. Mol. Catal. A: Chem., 314, 28-34 (2009).

[90] F. P. Netzer, A. Goldmann, G. Rosina and E. Bertel, Structure and surface chemistry of 5-membered cyclic molecules on $\mathrm{Rh}(111)$ : Cyclopentene and cyclopentadiene, Surf. Sci., 204, 387 (1988).

[91] F. P. Netzer, G. Rosina, E. Bertel and H. B. Saalfeld, UV photoelectron spectra of adsorbed cyclopentadienyl, J. Electron Spectrosc. Relat. Phenom., 46, 373-379 (1988).

[92] C. Waldfried, D. Welipitiya, C. W. Hutchings, H. S. V. de Silva, G. A. Gallup, P. A. Dowben, W. W. Pai, J. Zhang, J. F. Wendelken and N. M. Boag, Preferential bonding orientations of ferrocene on surfaces, J. Phys. Chem. B, 101, 9782 (1997).

[93] J. Choi and P. A. Dowben, Cobaltocene adsorption and dissociation on $\mathrm{Cu}(111)$, Surf. Sci., 600, 2997 (2006).

[94] K.-F. Braun, V. Iancu, N. Pertaya, K.-H. Rieder and S.-W. Hla, Decompositional incommensurate growth of ferrocene molecules on a $\mathrm{Au}(111)$ surface, Phys. Rev. Lett., 96, 246102 (2006).

[95] M. Sacchi, S. J. Jenkins, private communications.

[96] R. B. Moffett, Cyclopentadiene and 3-chlorocyclopentadiene, Org. Synth., 32, 41 (1952).

[97] M. H. Mohamed, L. L. Kesmodel, B. M. Hall and D. L. Mills, Surface phonon dispersion on $\mathrm{Cu}(111)$, Phys. Rev. B, 37, 2763 (1988).

[98] A. P. Jardine, J. Ellis and W. Allison, Effects of resolution and friction in the interpretation of QHAS measurements, J. Chem. Phys., 120, 8724 (2004).

[99] G. Alexandrowicz, A. P. Jardine, H. Hedgeland, W. Allison and J. Ellis, Onset of 3D Collective Surface Diffusion in the Presence of Lateral Interactions: $\mathrm{Na} / \mathrm{Cu}(001)$, Phys. Rev. Lett., 97, 156103 (2006).

[100] H. Hedgeland, P. Kole, H. Davies, A. P. Jardine, G. Alexandrowicz, W. Allison, J. Ellis, G. Fratesi and G. Brivio, Surface dynamics and friction of $\mathrm{K} / \mathrm{Cu}(001)$ characterized by helium-3 spin-echo and density functional theory, Phys. Rev. B, 80, 1-7 (2009). 
[101] H. Hedgeland, B. A. J. Lechner, F. E. Tuddenham, A. P. Jardine, W. Allison, J. Ellis, M. Sacchi, S. J. Jenkins and B. J. Hinch, Weak intermolecular interactions in an ionically bound molecular adsorbate: Cyclopentadienyl $/ \mathrm{Cu}(111)$, Phys. Rev. Lett., 106, 186101 (2011).

[102] M. Sacchi, S. Jenkins, H. Hedgeland, A. P. Jardine and B. J. Hinch, Electronic structure and bonding of an ionic molecular adsorbate: $\mathrm{c}_{-} \mathrm{C}_{5} \mathrm{H}_{5}$ on $\mathrm{Cu}(111), J$. Phys. Chem. B, 115, 16134-16141 (2011).

[103] S. J. Clark, M. D. Segall, C. J. Pickard, P. J. Hasnip, M. J. Probert, K. Refson and M. Payne, First principles methods using CASTEP, Z. Kristallogr., 220, 567 (2005).

[104] J. P. Perdew and Y. Wang, Accurate and simple analytic representation of the electron-gas correlation energy, Phys. Rev. B, 45, 13244 (1992).

[105] J. Topping, On the mutual potential energy of a plane network of doublets, Proc. Roy. Soc. London, 114, 726, 67 (1927).

[106] J. Ellis, A. P. Graham, R. Hoffmann and J. P. Toennies, Coverage dependence of the microscopic diffusion of $\mathrm{Na}$ atoms on the $\mathrm{Cu}(001)$ surface: A combined helium atom scattering experiment and molecular dynamics study, Phys. Rev. B, 63, 195408 (2001).

[107] D. Tang, D. McIlroy, X. Shi, C. Su and D. Heskett, The structure of Na overlayers on $\mathrm{Cu}(111)$ at room temperature, Surf. Sci., 255, L497 (1991).

[108] D. S. Sivia and J. Skilling, Data Analysis: A Bayesian Tutorial, Oxford University Press (2006).

[109] J. L. Vega, R. Guantes and S. Miret-Artés, Quasielastic and low vibrational lineshapes in atom-surface diffusion, J. Phys.: Condens. Matter, 16, S2879S2894 (2004).

[110] M. E. Tuckermann, Statistical Mechanics: Theory and Molecular Simulation, Oxford University Press (2010).

[111] J. Ellis, A. P. Graham and J. P. Toennies, Quasielastic Helium Atom Scattering from a Two-Dimensional Gas of Xe Atoms on Pt(111), Phys. Rev. Lett., 82, 5072-5075 (1999). 
[112] A. P. Jardine, H. Hedgeland, D. Ward, Y. Xiaoqing, W. Allison, J. Ellis and G. Alexandrowicz, Probing molecule-surface interactions through ultra-fast adsorbate dynamics: propane/Pt(111), New Journal of Physics, 10, 125026 (2008).

[113] R. A. Sánchez-Delgado, Organometallic Modeling of the Hydrodesulfurization and Hydrodenitrogenation Reactions, Kluwer Academic Publishers (2002).

[114] Y.-C. Liu, S.-J. Yang, T. C. Chuang and C.-C. Wang, Pyrrole self-assembled monolayer and orderly autopolymerization on chloride- and gold-containing nanocomplexes prepared by electrochemical methods, J. Electroanal. Chem., 570, 1-5 (2004).

[115] C. N. Sayre and D. M. Collard, Self-Assembled Monolayers of Pyrrole-Containing Alkanethiols on Gold, Langmuir, 11, 302-306 (1995).

[116] J. D. Stenger-Smith, Intrinsically electrically conducting polymers. Synthesis, characterization, and their applications, Prog. Polym. Sci., 23, 57-79 (1998).

[117] A. M. Fenelon and C. B. Breslin, The electrochemical synthesis of polypyrrole at a copper electrode: corrosion protection properties, Electrochim. Acta, 47, 4467-4476 (2002).

[118] W. Prissanaroon, N. Brack, P. Pigram and J. Liesegang, Electropolymerisation of pyrrole on copper in aqueous media, Synthetic Metals, 142, 25-34 (2004).

[119] B. Bak, D. Christensen, L. Hansen and J. Rastrup-Andersen, Microwave Determination of the Structure of Pyrrole, J. Chem. Phys., 24, 4, 720, ISSN 00219606 (1956).

[120] L. Nygaard, J. T. Nielsen, J. Kirchheiner, G. Maltesen, J. Rastrup-Andersen and G. O. Sørensen, Microwave spectra of isotopic pyrroles. Molecular structure, dipole moment, and ${ }^{14} \mathrm{~N}$ quadrupole coupling constants of pyrrole, J. Mol. Structure, 3, 491-506 (1969).

[121] B. A. Sexton, A vibrational and TDS study of the adsorption of pyrrole, furan and thiophene on $\mathrm{Cu}(100)$ : Evidence for $\pi$-bonded and inclined species, Surf. Sci., 163, 99-113 (1985).

[122] J. Gaudioso and W. Ho, Single-Molecule Vibrations, Conformational Changes, and Electronic Conductivity of Five-Membered Heterocycles, J. Am. Chem. Soc., 123, 10095-10098 (2001). 
[123] G. R. Schoofs and J. B. Benzinger, Reactions of pyrrole with Ni(100), Surf. Sci., 192, 373-382 (1987).

[124] C. J. Baddeley, C. Hardacre, R. M. Ormerod and R. M. Lambert, Chemisorption and decomposition of pyrrole on $\operatorname{Pd}(111)$, Surf. Sci., 369, 1-8 (1996).

[125] D. N. Futaba and S. Chiang, Predictions of scanning tunneling microscope images of furan and pyrrole on $\operatorname{Pd}(111)$, J. Vac. Sci. Technol. A, 15, 1295 (1997).

[126] T. E. Caldwell and D. P. Land, Desulfurization, deoxygenation and denitrogenation of heterocycles by a palladium surface: a mechanistic study of thiophene, furan and pyrrole on Pd (111) using laser-induced thermal desorption with Fourier-transform mass spectrometry, Polyhedron, 16, 3197 (1997).

[127] G. Tourillon, S. Raaen, T. A. Skotheim, M. Sagurton, R. Garrett and G. P. Williams, A near edge x-ray absorption fine structure study of the adsorption of pyrrole and N-methylpyrrole on $\operatorname{Pt}(111)$ : Orientation and dissociation of the adsorbed molecules, Surf. Sci., 184, L345-L354 (1987).

[128] F. P. Netzer, E. Bertel and A. Goldmann, Structure and surface chemistry of 5-membered cyclic molecule on Rh(111): Pyrrole, Surf. Sci., 199, 87 (1988).

[129] W. A. Abdallah and A. E. Nelson, Pyrrole adsorption and reaction on Mo(110) and C/N-Mo(110), Surf. Sci., 585, 113-122 (2005).

[130] W. A. Abdallah and A. E. Nelson, Density Functional Theory Study of Pyrrole Adsorption on Mo(110), J. Phys. Chem. B, 109, 10863-10870 (2005).

[131] P. A. Redhead, Thermal Desorption of Gases, Vacuum, 12, 203 (1962).

[132] S. Paterson, W. Allison, H. Hedgeland, J. Ellis and A. P. Jardine, Rotation and translational motion prior to self-assembly: Dynamics of ethanethiolate on $\mathrm{Cu}(111)$, Phys. Rev. Lett., 106, 256101 (2011).

[133] A. Tkatchenko and M. Scheffler, Accurate Molecular Van Der Waals Interactions from Ground-State Electron Density and Free-Atom Reference Data, Phys. Rev. Lett., 102, 073005 (2009).

[134] J. K. Nørskov and N. D. Lang, Effective-medium theory of chemical binding: Application to chemisorption, Phys. Rev. B, 21, 2131 (1980). 
[135] M. Manninen, J. K. Nørskov, M. J. Puska and C. Umrigar, Repulsive interaction of the helium atom with a metal surface, Phys. Rev. B, 29, 2314 (1984).

[136] G. Fratesi, G. Alexandrowicz, M. I. Trioni, G. P. Brivio and W. Allison, Crucial electronic contributions to measures of surface diffusion by He atom scattering, Phys. Rev. B, 77, 235444 (2008).

[137] G. Barbarella, M. Melucci and G. Sotgiu, The Versatile Thiophene: An Overview of Recent Research on Thiophene-Based Materials, Adv. Mater., 17, 1581-1593 (2005).

[138] P. K. Milligan, B. Murphy, D. Lennon, B. C. C. Cowie and M. Kadodwala, A complete structural study of the coverage dependence of the bonding of thiophene on $\mathrm{Cu}(111)$, J. Phys. Chem. B, 105, 140-148 (2001).

[139] J. A. Rodriguez and J. Hrbek, Interaction of sulfur with well-defined metal and oxide surfaces: Unraveling the mysteries behind catalyst poisoning and desulfurization, Acc. Chem. Res., 32, 9, 719 (1999).

[140] A. K. Neyestanaki, F. Klingstedt, T. Salmi and D. Y. Murzin, Deactivation of postcombustion catalysts, a review, Fuel, 83, 395-408 (2004).

[141] V. Schomaker and L. Pauling, The electron diffraction investigation of the structure of benzene, pyridine, pyrazine, butadiene-1,3, cyclopentadiene, furan, pyrrole, and thiophene, J. Am. Chem. Soc., 61, 1769 (1939).

[142] B. Bak, D. Christensen, L. Hansen-Nygaard and J. Rastrup-Andersen, The structure of thiophene, Journal of Molecular Spectroscopy, 7, 58-63 (1961).

[143] J. Stöhr, J. L. Gland, E. B. Kollin, R. J. Koestner, A. L. Johnson, E. L. Muetterties and F. Sette, Desulfurization and structural transformation of thiophene on the Pt(111) surface, Phys. Rev. Lett., 53, 2161 (1984).

[144] A. Imanishi, S. Yagib, T. Yokoyama, Y. Kitajima and T. Ohta, Structural and electronic properties of adsorbed $\mathrm{C}_{4} \mathrm{H}_{4} \mathrm{~S}$ on $\mathrm{Cu}(100)$ and $\mathrm{Ni}(100)$ studied by $\mathrm{S}$ K-XAFS and S-1s XPS, J. Electron Spectrosc. Relat. Phenom., 80, 151-154 (1996).

[145] N. V. Richardson and J. C. Campuzano, The adsorption of thiophene on a $\mathrm{Cu}(111)$ surface, Vacuum, 31, 449-451 (1981). 
[146] A. Imanishi, T. Yokoyama, Y. Kitajima and T. Ohta, Structural and electronic properties of adsorbed thiophene on $\mathrm{Cu}(111)$ studied by $\mathrm{S}$ K-edge X-ray absorption spectroscopy, Bull. Chem. Soc. Jpn., 71, 831-835 (1998).

[147] P. Milligan, J. McNamarra, B. Murphy, B. C. C. Cowie, D. Lennon and M. Kadodwala, A NIXSW and NEXAFS investigation of thiophene on $\mathrm{Cu}(111)$, Surf. Sci., 412/413, 166-173 (1998).

[148] S. Paterson, C. J. Aas and A. P. Jardine, Quasi-elastic lineshapes for atoms and simple molecules undergoing jump rotation on surfaces, Surf. Sci., 606, 426-434 (2012).

[149] G. Alexandrowicz, A. P. Jardine, P. Fouquet, S. Dworski, W. Allison and J. Ellis, Observation of microscopic $\mathrm{CO}$ dynamics on $\mathrm{Cu}(001)$ using ${ }^{3} \mathrm{He}$ spin-echo spectroscopy, Phys. Rev. Lett., 93, 156101-156103 (2004).

[150] A. S. de Wijn and A. Fasolino, Relating chaos to deterministic diffusion of a molecule adsorbed on a surface, J. Phys.: Condens. Matter, 21, 264002 (2009).

[151] A. S. de Wijn, Internal degrees of freedom and transport of benzene on graphite, Phys. Rev. E, 84, 011610 (2011). 